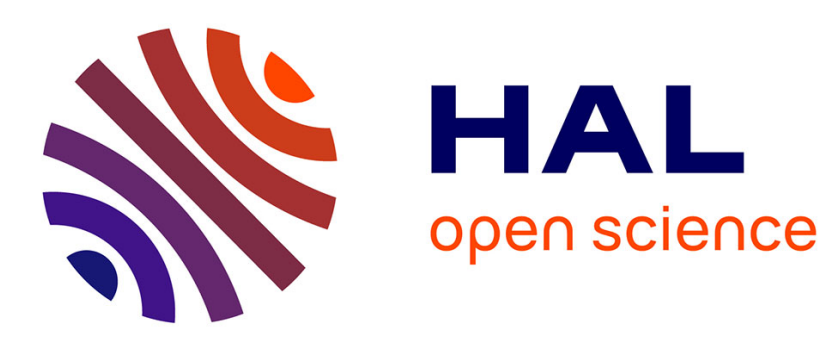

\title{
Calibration and performance test of the Very-Front-End electronics for the CMS electromagnetic calorimeter
} Jan Blaha

\section{To cite this version:}

Jan Blaha. Calibration and performance test of the Very-Front-End electronics for the CMS electromagnetic calorimeter. High Energy Physics - Experiment [hep-ex]. Université Claude Bernard - Lyon I, 2008. English. NNT : . tel-00387489

\section{HAL Id: tel-00387489 \\ https://theses.hal.science/tel-00387489}

Submitted on 25 May 2009

HAL is a multi-disciplinary open access archive for the deposit and dissemination of scientific research documents, whether they are published or not. The documents may come from teaching and research institutions in France or abroad, or from public or private research centers.
L'archive ouverte pluridisciplinaire HAL, est destinée au dépôt et à la diffusion de documents scientifiques de niveau recherche, publiés ou non, émanant des établissements d'enseignement et de recherche français ou étrangers, des laboratoires publics ou privés. 


\section{Thèse}

présentée devant

l’Université Claude Bernard Lyon-I

pour l'obtention du

DIPLOME de DOCTORAT

Spécialité : Physique des Particules

(arrêté du 7 août 2006)

Jan BLAHA

\section{Calibration et études des performances de l'électronique frontale du calorimètre électromagnétique de l'expérience CMS auprès du collisionneur LHC}

Soutenue le 20 mai 2008

devant la Commission d'Examen

\begin{tabular}{|c|c|c|c|c|}
\hline \multirow[t]{7}{*}{ Jury : } & M. & H. & El Mamouni & Directeur de Thèse \\
\hline & M. & M. & Finger & Directeur de Thèse \\
\hline & M. & J. & Fay & Président du Jury \\
\hline & M & P. & Jarry & Rapporteur \\
\hline & M. & V. & Vrba & Rapporteur \\
\hline & M. & J.-P. & Ernenwein & \\
\hline & M. & V. & Petråček & \\
\hline
\end{tabular}




\title{
THESE
}

présentée

devant l'UNIVERSITE CLAUDE BERNARD - LYON 1

pour l'obtention

du DIPLOME DE DOCTORAT

(arrêté du 7 aoû 2006 et arrêté du 6 janvier 2005)

présentée et soutenue publiquement le

20 mai 2008

par

Jan BLAHA

\section{Calibration et études des performances de l'électronique frontale du calorimètre électromagnétique de l'expérience CMS auprès du collisionneur $\mathrm{LHC}$}

\author{
Directeur de thèse : M. EL MAMOUNI Houmani \\ M. FINGER Miroslav \\ Jury : M. FAY Jean, president \\ M. JARRY Patrick, rapporteur \\ M. VRBA Václav, rapporteur \\ M. ERNENWEIN Jean-Pierre \\ M. PETRÁČEK Vojtěch
}





\title{
Calibration and performance test of the Very-Front-End electronics for the CMS electromagnetic calorimeter
}

\author{
Jan Blaha
}

A Thesis presented for the degree of Doctor of Philosophy

\author{
Fakulta jaderná a fyzikálně inženýrská \\ Ceské vysoké učení technické v Praze \\ Institut de Physique Nucléaire de Lyon - IN2P3/CNRS \\ Université Claude Bernard Lyon 1
}

May 2008 

Dedicated to my parents and brother 



\title{
Calibration and performance test of the Very-Front-End electronics for the CMS electromagnetic calorimeter
}

\author{
Jan Blaha \\ Submitted for the degree of Doctor of Philosophy \\ May 2008
}

\begin{abstract}
A Very-Front-End (VFE) card is an important part of the on-detector read-out electronics of the CMS (Compact Muon Solenoid) electromagnetic calorimeter that is built of $\sim 76,000$ radiation hard scintillating crystals $\mathrm{PbWO}_{4}$ and operates on the Large Hadron Collider (LHC) at CERN. Almost 16,000 VFE cards which shape, amplify and digitalize incoming signals from photodetectors generated by interacting particles.

Since any maintenance of any part of the calorimeter is not possible during the 10-year lifetime of the experiment, the extensive screening program was employed throughout the whole manufacture process. As a part of readout electronics QA program, the systems for burn-in and precise calibration of the VFE boards were developed and successfully used at IPN Lyon. In addition to functionality tests, all relevant electrical properties of each card were measured and analyzed in detail to obtain their full characterization and to build a database with all required parameters which will serve for the initial calibration of the whole calorimeter.

In order to evaluate the calorimeter performance and also to deliver the most precise calibration constants, several fully equipped supermodules were extensively studied and calibrated during the test beam campaigns at CERN. As an important part of these tests, accurate studies of the electronics noise and relative gains, which are needed for measurement in high energy range, were carried out to optimize amplitude reconstruction procedure and thus improve the precision of the calorimeter energy determination.

The heart of the thesis consists of the calibration of all VFE boards, including optimization of the laboratory calibration system and precise analysis of measured values to delivered desired calibration constants. The second half of the thesis is focused on the accurate evaluation and optimization of the read-out electronics in real data taking conditions. The results obtained in the laboratory at IPN Lyon as well as during test beam at CERN confirm the high quality of the boards production and show that the CMS detector specifications are reached.
\end{abstract}





\title{
Calibration et études des performances de l'électronique frontale du calorimètre électromagnétique de l'expérience CMS auprès du collisionneur LHC
}

\author{
Jan Blaha \\ Présentée pour l'obtention du grade de Docteur \\ mai 2008
}

Résumé

La carte VFE (Very-Front-End) est une partie importante de l'électronique de lecture embarquée du calorimètre électromagnétique de CMS (Compact Muon Solenoid) fait de $~ 76,000$ cristaux scintillants durs aux radiations de tungstate de plomb $\left(\mathrm{PbWO}_{4}\right)$ installé sur le grand Colisionneur de Hadron (LHC) au CERN. Presque 16000 cartes VFE qui mettent en forme, amplifient et numérisent les signaux émis par les détecteurs photoélectriques lors de l'interaction des particules.

Puisqu'aucune maintenance des parties embarquées du calorimètre n'est possible durant les dix ans de la vie de l'expérience, un programme extensif de garantie de qualité a été mis en place tout au long du processus de construction. L'application de ce programme à l'électronique de lecture comprend les systèmes de déverminage et de calibration précise des VFE, développés et utilisés avec succès à l'IPN de Lyon. Outre les tests de fonctionnalité, toutes les propriétés électriques de chaque carte ont été mesurées et analysées en détail pour obtenir leur pleine caractérisation et établir une base de données avec tous les paramètres nécessaires à la calibration initiale de l'ensemble du calorimètre.

Afin d'évaluer les performances du calorimètre et fournir les constantes de calibration les plus précises, plusieurs supermodules entièrement équipés ont été intensivement étudiés et calibrés pendant les campagnes de tests en faisceau au CERN. Notamment l'étude précise du bruit de l'électronique et la détermination des gains relatifs, nécessaires dans les mesures de haute énergie, ont été effectués pour optimiser le procédé de reconstruction d'amplitude et améliorer ainsi la précision de la mesure d'énergie du calorimètre.

La première partie de la thèse comprend la calibration de toutes les cartes VFE, y compris l'optimisation du système de calibration en laboratoire et l'analyse précise des valeurs mesurées afin de fournir les constantes de calibration requises. La deuxième moitié de la thèse concerne l'évaluation et l'optimisation précises de l'électronique de lecture avec des données obtenues dans des conditions réelles. Les résultats obtenus en laboratoire à l'IPN Lyon comme avec le faisceau test au CERN confirment la qualité de la production de cartes et prouvent que les caractéristiques nécessaires au fonctionnement du détecteur de CMS ont été atteintes. 



\section{Declaration}

The work in this thesis is based on joint research carried out at the CMS Experiment Group at the Institut de Physique Nucléaire de Lyon - IN2P3/CNRS, the Université Claude Bernard and the Czech Technical University in Prague. No part of this thesis has been submitted elsewhere for any other degree or qualification and it is all my own work unless referenced to the contrary in the text.

Copyright (c) 2008 by Jan Blaha.

"The copyright of this thesis rests with the author. No quotations from it should be published without the author's prior written consent and information derived from it should be acknowledged". 



\section{Acknowledgements}

It is a great pleasure for me to express my gratitude to all the people who in one way or another have contributed to made this thesis possible. Among them there are my colleagues from IPNL, CTU, the CMS collaboration as well as my friends. I cannot mention all by names, but, on the following lines, I wish to highlight some of them.

First of all, I would like to express my thanks and gratitude to Jean FAY, head of our group, with who I had the opportunity of working closely and who guided and supported me continuously during the whole process of my thesis. I am also delighted to include Christophe COMBARET and Georges MAURELLI, engineers who were in charge of the ECAL readout electronics at IPNL, who were always open for discussion and who made a cheerful working environment.

Moreover, I am very grateful with my thesis supervisors, Houmani EL MAMOUNI and Miroslav FINGER, who were pleased to help and stood besides me throughout all the administration process of the joint doctoral program between the French and Czech universities. I would also like to appreciate my referees, Patrick JARRY and Václav VRBA, for their careful reading of my thesis and for providing helpful comments and suggestions which increased the quality of my work.

Finally, I love to thank to my family for their unconditional support. 



\section{Contents}

$\begin{array}{ll}\text { Abstract } & 7\end{array}$

$\begin{array}{lr}\text { Résumé } & 9\end{array}$

$\begin{array}{ll}\text { Declaration } & 11\end{array}$

$\begin{array}{ll}\text { Acknowledgements } & 13\end{array}$

$\begin{array}{ll}\text { Introduction } & 33\end{array}$

$1 \quad$ Large Hadron Collider and CMS experiment $\quad 37$

1.1 Introduction . . . . . . . . . . . . . . . . . . . . 37

1.2 Large Hadron Collider . . . . . . . . . . . . . . . . . . . . . 37

1.2 .1 LHC physics potential . . . . . . . . . . . . . . . . . . 39

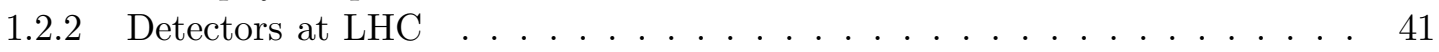

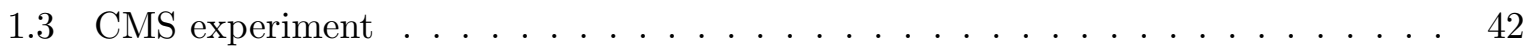

1.3 .1 Tracker . . . . . . . . . . . . . . . . . . . 42

1.3.2 Electromagnetic calorimeter . . . . . . . . . . . . . . 45

1.3.3 Hadronic calorimeter . . . . . . . . . . . . . . . . . . . . . 45

1.3.4 Superconducting solenoid . . . . . . . . . . . . . . . . . . 46

1.3.5 Muon system . . . . . . . . . . . . . . . . . . . . 48

1.4 Conclusion . . . . . . . . . . . . . . . . . . . . . . . . . 49

2 Electromagnetic calorimeter $\quad \mathbf{5 3}$

2.1 Introduction . . . . . . . . . . . . . . . . . . . 53

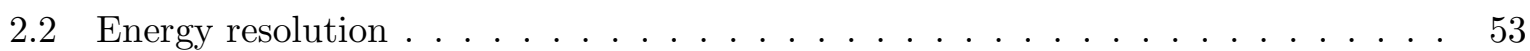

2.2 .1 Stochastic term . . . . . . . . . . . . . . . . 53

2.2 .2 Noise term . . . . . . . . . . . . . . . . . . 55

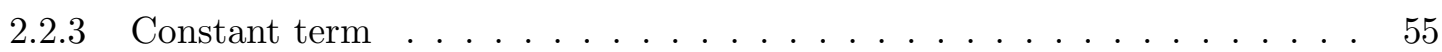

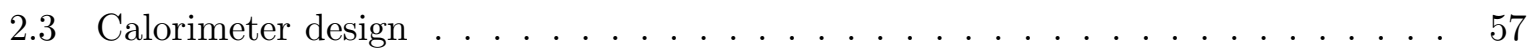

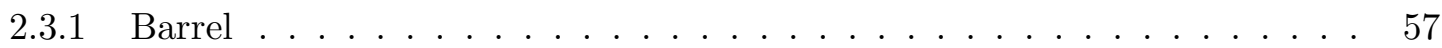

2.3 .2 Endcaps . . . . . . . . . . . . . . . . . . . . . . . . . 58

2.3 .3 Pre-Shower . . . . . . . . . . . . . . . . . . . . . . 58

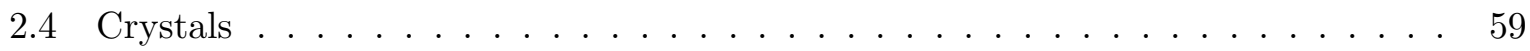

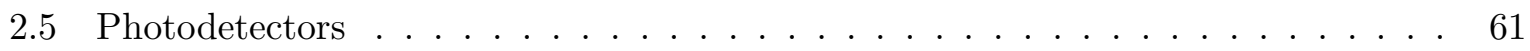

2.5.1 Avalanche photodiodes . . . . . . . . . . . . . . . . . . 61

2.5.2 Vacuum phototriodes ... . . . . . . . . . . . . 64

2.6 Crystal transparency monitoring . . . . . . . . . . . . . . . . 67 


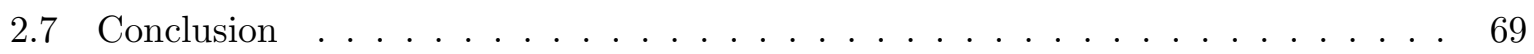

$\begin{array}{lll}3 & \text { Readout electronics } & \mathbf{7 3}\end{array}$

3.1 Introduction . . . . . . . . . . . . . . . . . . . . 73

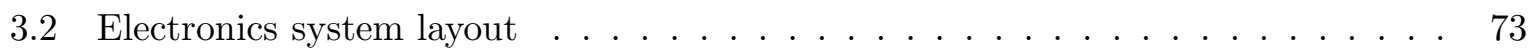

3.3 On-detector electronics . . . . . . . . . . . . . . . . . . . . . 74

3.3 .1 Motherboard . . . . . . . . . . . . . . . . . 74

3.3.2 Low voltage regulator board (LVR) . . . . . . . . . . . . 75

3.3 .3 Very-front-end board $(\mathrm{VFE}) \ldots \ldots \ldots \ldots \ldots$. . . . . . . . . . . . . . . . 76

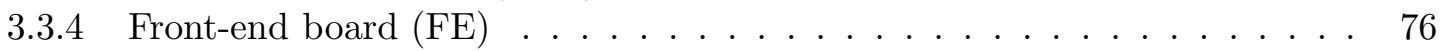

3.3 .5 Token $\operatorname{ring}$ board $(\mathrm{TRB}) \ldots \ldots \ldots \ldots \ldots$. . . . . . . . . . . . . 82

3.4 Off-detector electronics . . . . . . . . . . . . . . . . . . . . 83

3.4.1 Clock and control system (CCS) . . . . . . . . . . . . . 84

3.4 .2 Trigger concentrator card $(\mathrm{TCC}) \ldots \ldots \ldots \ldots . \ldots \ldots 4$

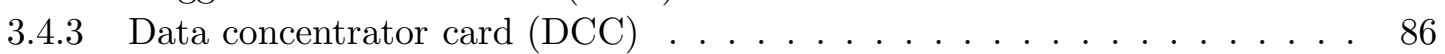

3.4 .4 Selective readout processor $(\mathrm{SRP}) \ldots \ldots \ldots \ldots$. . . . . . 86

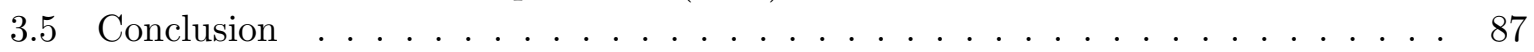

4 Very-Front-End electronics $\quad 93$

4.1 Introduction . . . . . . . . . . . . . . . . . . . . . . . . 93

4.2 Very-front-end card . . . . . . . . . . . . . . . . . . . 93

4.3 Multi-Gain Pre-Amplifier . . . . . . . . . . . . . . . . . . 94

4.3 .1 Electronic architecture . . . . . . . . . . . . . . . 96

4.3 .2 Test pulse unit . . . . . . . . . . . . . . . . 96

4.3.3 Performance characteristics . . . . . . . . . . . . . . . . . . 97

4.3 .4 Production procedure . . . . . . . . . . . . . . . . . . . 99

4.4 Analogue-to-Digital Converter . . . . . . . . . . . . . . . . . . 100

4.4.1 Electronic architecture . . . . . . . . . . . . . . . . . 100

4.4.2 Operation modes . . . . . . . . . . . . . . . . . . . 102

4.4 .3 Performance characteristics . . . . . . . . . . . . . . . . 103

4.4 .4 Radiation tolerance . . . . . . . . . . . . . . . . . . . 105

4.5 LVDS_RX adapters . . . . . . . . . . . . . . . . . . . 107

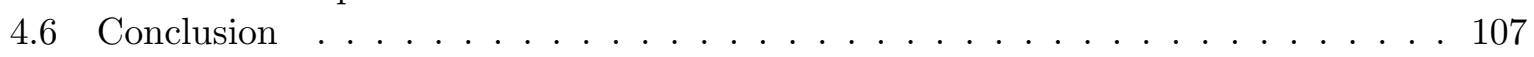

5 VFE Calibration and performance 111

5.1 Introduction . . . . . . . . . . . . . . . . . . . . . 111

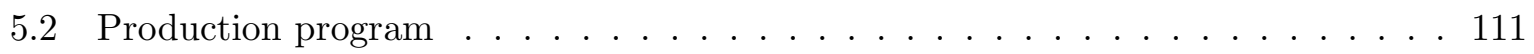

5.3 Automatic optical inspection . . . . . . . . . . . . . . . . . 111

5.4 Power-on test . . . . . . . . . . . . . . . . . . . . . . . . 112

5.4 .1 Functional tests . . . . . . . . . . . . . . . . . . . . . . 112

5.4 .2 Power consumptions . . . . . . . . . . . . . . . . . . 113

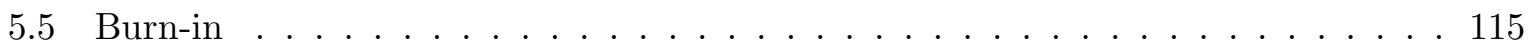

5.5.1 VFE burn-in procedure . . . . . . . . . . . . . . . 117

5.6 VFE calibration and performance . . . . . . . . . . . . . . . 119

5.6 .1 Test bench . . . . . . . . . . . . . . . . . . . . . . . . 119

5.6 .2 Calibration procedure . . . . . . . . . . . . . . . . . 121

5.6 .3 Calibration results . . . . . . . . . . . . . . . . . . . . . 124

5.6 .4 Gain ratios . . . . . . . . . . . . . . . . . . . . . . 127 
5.6.5 Pedestal and electronic noise . . . . . . . . . . . . . . . 130

5.6 .6 Linearity . . . . . . . . . . . . . . . . . . . . 133

5.6 .7 Precise linearity study . . . . . . . . . . . . . . . . . . . 134

5.6 .8 Electronic saturation . . . . . . . . . . . . . . . . . . . 137

5.6 .9 Temperature dependence measurement . . . . . . . . . . . . . . . . . 138

5.7 Conclusion . . . . . . . . . . . . . . . . . . . . . . . 139

$6 \quad$ ECAL test beam performance $\quad 143$

6.1 Introduction . . . . . . . . . . . . . . . . . . . . . 143

6.2 Test beam objectives . . . . . . . . . . . . . . . . . . . . . 144

$6.3 \mathrm{H} 4$ test beam line . . . . . . . . . . . . . . . . . . . . . 144

6.4 Test beam set-up . . . . . . . . . . . . . . . . . . . 146

6.5 Reconstruction of the signal amplitude . . . . . . . . . . . . . . . 148

6.5 .1 The weights method . . . . . . . . . . . . . . . . . . 149

6.5.2 Weights optimization . . . . . . . . . . . . . . . 150

6.6 Noise Performance . . . . . . . . . . . . . . . . . . . . . . . . . . . . 152

6.6 .1 Pile-up noise . . . . . . . . . . . . . . . . . . . 153

6.6 .2 Electronics noise . . . . . . . . . . . . . . . . . . . . 154

6.6 .3 Noise evaluation definitions . . . . . . . . . . . . . . . . . 155

6.6.4 Systematic studies of pedestal end electronic noise . . . . . . . . . . . 155

6.6.5 Correlated noise . . . . . . . . . . . . . . . . . . . 158

6.6.6 Noise reduction in the amplitude reconstruction . . . . . . . . . . . . . 162

6.6 .7 Noise stability over time . . . . . . . . . . . . . . . . . 162

6.6 .8 Conclusion on noise analysis . . . . . . . . . . . . . . . . . . 164

6.7 Gain ratio measurement . . . . . . . . . . . . . . . . . . . . 164

6.7.1 Check on systematic effects . . . . . . . . . . . . . . 166

6.7.2 Gain ratio measured with test pulse . . . . . . . . . . . . 170

6.7.3 Gain ratio measured with laser . . . . . . . . . . . . . . . . . . . 172

6.7.4 Gain ratio measured with beam . . . . . . . . . . . . . . . . . . . . 174

6.7.5 Energy dependence measurement . . . . . . . . . . . . . . 178

6.7.6 Stability of the test pulse gain ratio over time . . . . . . . . . . . . 180

6.7.7 Conclusion on gain ratio studies . . . . . . . . . . . . . . . 180

6.8 Energy resolution . . . . . . . . . . . . . . . . . . . . . . . . . 182

6.9 Conclusion . . . . . . . . . . . . . . . . . . . . . . 183

$\begin{array}{ll}\text { Conclusions } & 187\end{array}$ 



\section{List of Figures}

1.1 Production cross-section for various processes at hadron $\mathrm{p}-\mathrm{p}$ and $\mathrm{p}-\overline{\mathrm{p}}$ colliders as a function of the machine centre-of-mass energy [3] . . . . . . . . . . . . .

1.2 Expected statistical significance with an integrated luminosity of $30 \mathrm{fb}^{-1}$ for the SM Higgs boson as a function of $\mathrm{m}_{H}$ in the CMS experiment [4]. . . . . . . . . 40

1.3 Left: The leading order cross section for the SM Higgs boson as a function of $\mathrm{m}_{H}$. Right: Branching fractions for the SM Higgs boson as a function of $\mathrm{m}_{H}$ [4]. . . . 41

1.4 Overall view of the CMS detector. . . . . . . . . . . . . . . . 42

1.5 Longitudinal view of one quadrant of the CMS tracking system configuration with respect to the collision point. The TIB, TOB, TID, and TED denote the Trigger Inner and Outer Barrels, Tracker Inner Disk, and Tracker Endcap. . . . . . . . . 43

1.6 An artistic view of the pixel detector showing its barrel and endcap parts. . . . . 43

1.7 Top: Resolution of transverse momentum (left), transverse impact parameter (middle), and longitudinal impact parameter (right) for single muons with transverse momenta of 1,10 and $100 \mathrm{GeV}$. Bottom: Global track reconstruction efficiency for muons (left) and pions (right) of transverse momenta of 1, 10 and

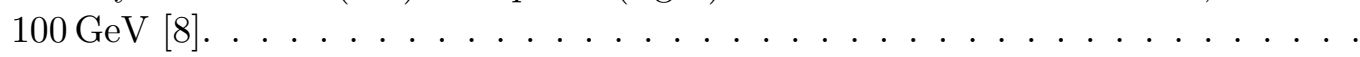

1.8 Longitudinal view of the CMS detector showing the locations of the hadron barrel (HB), endcap (HE), outer (HO) and forward (HF) calorimeters. . . . . . . . .

1.9 Left: The jet transverse-energy resolution as a function of the simulated jet transverse energy for barrel jets $(|\eta|<1.4)$, endcap jets $(1.4<|\eta|<3.0)$, and very forward jets $(3.0<|\eta|<5.0)$. Right: Energy resolution as a function of energy for test beam pions compared to two different GEANT4 simulations. . . . . . . . .

1.10 Layout of one quarter of the CMS muon system showing deployment of Drift Tubes (DTs), Cathode Strip Chambers (CSCs) and Resistive Plate Chambers (RPCs) integrated into the magnet return yoke. . . . . . . . . . . . . .

2.1 Total and different contributions to the energy resolution of the calorimeter. The curve labeled "intristic" includes both the shower containment and the constant term. The curve named "photo" represents stochastic contributions. . . . . . . . 54

2.2 Overall view (top) and the one quadrant longitudinal section (bottom) of the CMS ECAL showing the arrangement of crystal modules, supermodules and endcaps. Here SE is the acronym for the pre-shower detector. . . . . . . . . . .

2.3 All barrel supermodules mounted in their final possition inside the CMS hadron calorimeter. . . . . . . . . . . . . . . . 57

2.4 The schematic longitudinal cross-section of the SE, with the two $1 \mathrm{~cm}$ thicknesses of foam and the heating films $[6] \ldots \ldots \ldots \ldots$ 
2.5 Top: Progress in the longitudinal transmission spectrum of the $\mathrm{PbWO}_{4}$ crystal between the years 1995 and 1997 in comparison with maximum achievable transmission [1]. Bottom: Temperature dependency of the light yield and temperature coefficient of the $\mathrm{PbWO}_{4}$ crystal $[12] \ldots \ldots \ldots \ldots \ldots$

2.6 Left: A photography of a capsule assembled with two APDs. Right: Schematic view of principle of an APD. . . . . . . . . . . . . . .

2.7 Left: Distribution of the APD bias voltage at gain 50. The structure in the distribution is caused by the APD pairing process in $5 \mathrm{~V}$ bins. Right: Correlation between the bias voltage at gain 50 for the ADPs and the capsules. . . . . . . .

2.8 Left: A photograph of the CMS vacuum phototriodes (VPT). Right: Schematic view of the VPT . . . . . . . . . . . . . . . . . 65

2.9 Relative gain $G / G_{0}$ and excess noise factor $F$ as a function of the magnetic field [25]. 66

2.10 A schematic overview of the CMS ECAL light monitoring system. . . . . . . . 67

2.11 Left: The evolution of electron and laser responses as a function of time. Right: Crystal response to electrons $\left(\mathrm{S} / \mathrm{S}_{0}\right)$ versus response to laser light injected $\left(\mathrm{R} / \mathrm{R}_{0}\right)$ during irradiation and recovery phases $[28] \ldots \ldots \ldots \ldots 68$

3.1 Schematic view of one barrel trigger tower as a basic block of the on-detector readout electronics reading and processing a $5 \times 5$ crystal array. . . . . . . . .

3.2 Schematic view of the Low Voltage Regulator (LVR) prototype architecture powering five VFE boards and one FE board. . . . . . . . . . . . . . 75

3.3 Photograph of the Very-Front-End (VFE) card showing the five channels with MGPA, ADC, and two buffers each. . . . . . . . . . . . 77

3.4 Left: Photograph of the barrel FE board front side showing 7 FENIX chips, CCU chip and two GOHs connectors. Right: Back side of the barrel FE board with connectors for five VFE boards (five $\times 2$ connectors) and one for LVR board. . . . 77

3.5 Schematic block diagram of the ECAL on-detector readout electronics system. . 78

3.6 Block diagrams of the FENIX chips with indication of their operation modes for the barrel (left) and the endcap (right) versions of the FE board. . . . . . . . . . 79

3.7 Left: Triple redundant configuration register. Right: Triple redundant Finite State Machine register. Both are implemented in the FENIX chips in order to protect against the SEU. . . . . . . . . . . . . . . . .

3.8 Schematic diagram of the token ring architecture serving eight FE boards. The token link board is also shown with $2 \times 2$ optical links to the off-detector CCS mFEC card. . . . . . . . . . . . . . . . . . . . . . . .

3.9 Schematic block diagram of the ECAL off-detector electronics system architecture comprising four dedicated boards. . . . . . . . . . . . . . .

3.10 Block diagram of the architecture of the Clock and Control System (CCS) board of the ECAL off-detector electronics system. . . . . . . . . . . . . . . 85

3.11 Block diagram of the architecture of the endcap Trigger Concentrator Card (TCC) of the ECAL off-detector electronics system. . . . . . . . . . . . . . 85

3.12 Block diagram of the architecture of the Data Concentrator Card (DCC) of the ECAL off-detector electronics system. For the abbreviations see text. . . . . . . .

4.1 Schematic representation of one channel of the Very-Front-End (VFE) architecture comprising the MGPA, 12-bit multi-channel ADC AD41240 and two level adapters LVDS-RX. . . . . . . . . . . . . . . . . . 
4.2 Left: Schematic drawing of the MGPA architecture. Components inside the shaded area are on-chip, those outside are external. Right: Photograph of the MGPA die with internal circuitry locations indicated [4] . . . . . . . . . 95

4.3 MGPA pulse shape matching across and between ranges [4] . . . . . . . . . 97

4.4 MGPA non-linearity measured over the full dynamic range for all three gains [4]. 98

4.5 MGPA non-linearity measured for different values of gain-stage bias currents for the high gain channel [5]. Similar results were found also for the low and middle

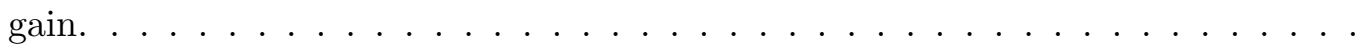

4.6 Left: Schematic block diagram of the AD41240 showing two ADC macro cells and the range selection logic. $\mathrm{V}_{b g}$ and $\mathrm{V}_{c m}$ blocks represent the bandgap reference generator and the common mode voltage generator, respectively. Right: Microphotograph of the chip with highlighted main functional blocks [10] . . . 100

4.7 Schematic of the ADC macro cell - dual 12 bit data converter [9]. . . . . . . . . 101

4.8 Typical spectrum of the differential non linearity (top) and integral non linearity (below) for the input frequency of $2.5 \mathrm{MHz}$ for one ADC channel [10]. . . . . . . 104

4.9 Power spectral density versus frequency for the input frequency of $2.5 \mathrm{MHz}$ for one ADC channel $[10] \ldots \ldots$. . . . . . . . . . . . . . . . 106

5.1 Block diagram of the power-on test system showing Power On Test Board (POTB) and plugged VFE card under test $[1] . \ldots \ldots \ldots \ldots$. . . . . . . . . 11

5.2 Distributions of the power supply, analog, digital and buffers voltage and currents measured for all 12,887 tested barrel VFE cards [3] . . . . . . . . . . . . . . . 114

5.3 The bathtub curve. . . . . . . . . . . . . . . . . . . . 116

5.4 Block diagram of the burn-in system showing two (out of 300) VFE cards under test. . . . . . . . . . . . . . . . . . . . . . . . . 118

5.5 Left: The set-up of the burn-in system at IPN Lyon. Other 150 VFE cards can be plugged in the back of the system. Right: Detailed view of the plugged VFE cards. . . . . . . . . . . . . . . . . . . 118

5.6 Schematic block diagram of the calibration test system developed and used at IPN Lyon and the test board with its internal main functionality parts. . . . . . 120

5.7 Photograph of the test board card (right) and the calibration system (left) for the VFE cards developed and used at IPN Lyon. . . . . . . . . . . . . . . 120

5.8 Typical injected charge pulses as VFE output in ADC counts for barrel (top) and endcap VFE (bottom) cards for gain 1, 6 , and 12. The lines are only to guide the

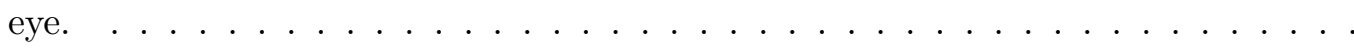

5.9 Relative difference between the theoretical and measured attenuation for each $1 \mathrm{~dB}$ step of the attenuator. The internal structure of the attenuator, where every attenuation is made as a combination of $1,2,4,8,16$, and $32 \mathrm{~dB}$ attenuation units. 124

5.10 VFE outputs in ADC counts as a function injected charge for a typical channel of the barrel (top) and endcap VFE (below) cards representing calibration curves for the three gains 1, 6, and 12. A charge of $4 \mathrm{pC}$ for barrel and 0.48 or $0.98 \mathrm{pC}$ for endcap VFE cards are common for all three gains and allows to perform a direct gain ratio measurement. . . . . . . . . . . . . . .

5.11 Slope distributions of the injected charges versus pulse height in ADC counts/pC for all the barrel (left) and all the endcap (right) VFE cards and the three gains

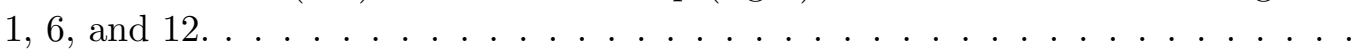


5.12 Offset distributions of the injected charges versus pulse height in ADC counts for all the barrel (left) and all the endcap (right) VFE cards and the three gains 1,

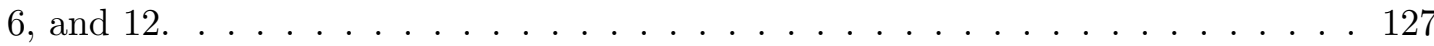

5.13 Slope distributions of the injected charges versus pulse height in ADC counts/pC for gain 12 for all the barrel VFE cards before and after the test board and time intercalibration. . . . . . . . . . . . . . . . . . . 128

5.14 Gain ratio $(12 / 6,12 / 1$, and 6/1) distributions for all the barrel (left) and endcap (right) VFE cards. . . . . . . . . . . . . . . . . . . . . . . 129

5.15 Distribution of the gain ratio 12/6 extracted from the slope (green) and measured using a test pulse charge of $4.1 \mathrm{pC}$ (blue) on the calibration bench at IPN Lyon for $340 \mathrm{VFE}$ cards (1,700 channels) which are now assembled in the supermodule SM17. . . . . . . . . . . . . . . . . . . .

5.16 Correlation (top) and relative difference (bottom) between the gain ratio 12/6 extracted from the slope and measured by the test pulse. The gain ratio was determined on the calibration bench at IPN Lyon for 340 VFE cards (1,700 channels) which are now assembled in the supermodule SM17. . . . . . . . . . . . .

5.17 Pedestal versus DAC value for five channels of a barrel VFE card, gain 1. The channel 1 does not work correctly, hence this VFE card has been rejected. . . . . 132

5.18 The noise distribution measured on the calibration bench for 8,992 barrel VFE cards in ADC counts for gains 1,6 , and 12. . . . . . . . . . . . 132

5.19 Residuals value delivered from the linear fit of the VFE response on the injected charge versus the injected charge for all the three gains for all the barrel (left) and endcap (right) VFE cards. . . . . . . . . . . . . . . . . 133

5.20 Experimental setup for the precise linearity study. . . . . . . . . . . . . . . . 134

5.21 Left: VFE response as a function of the injected delivered by the pulse squared generator for all the three gains. Right: The residuals from the linear fit versus injected charge. . . . . . . . . . . . . . . . .

5.22 Relative difference between pulse height (as a response of the injected charge delivered from the generator and by help of the attenuator) as a function of the charge for 30 measured channels (top) and for the mean over these channels (bottom). . . . . . . . . . . . . . . . . .

5.23 Left: VFE response as a function of the injected delivered by the pulse squared generator for all the three gains. Right: The residuals from the linear fit versus injected charge. . . . . . . . . . . . . . . . . .

5.24 ADC output for different injected charge in a single channel (left) and linearity plot for five channels of one barrel VFE board (right) . . . . . . . . . . . . 137

5.25 Top: The slope as a function of temperature in gain 12. Bottom: Coefficient of the temperature dependence for all three gains. The measurement performed on 6 reference VFE cards (30 channels) . . . . . . . . . . . . . . . . . . .

6.1 Three different settings of the $\mathrm{H} 4$ beam line configuration at CERN which were used during ECAL beam campaigns $[3] . \ldots \ldots \ldots \ldots$

6.2 A supermodule mounted on the remotely controlled movable table allowing to direct the high energy electrons beams to the chosen crystal. The table was installed at H4 SPS beam area at CERN. . . . . . . . . . . . . . . . . 147

6.3 A schematic view of the position of the hodoscope planes within the experimental set-up in the $\mathrm{H} 4$ beam line at CERN. . . . . . . . . . . . . . . . . . . . 147 
6.4 Beam intensity profiles along $x$ (left) and $y$ (right) directions measured by the scintillating fiber hodoscope for an electron beam of $120 \mathrm{GeV}$. . . . . . . . . . . . 148

6.5 Left: Digital representation of the electronics pulse as a series of 10 samples. Each sample is recorded every $25 \mathrm{~ns}=1$ clock unit. The pedestal was not subtracted and the analytic fit represents a true shape of the signal pulse. Right: Profile histogram of the signal pulse obtained from a $120 \mathrm{GeV}$ electron run. The peaking time $T_{\max }$, the pedestal $P$, and the amplitude $A$ of the signal are also highlighted. 149

6.6 Energy reconstructed in the $3 \times 3$ crystal arrays with the beam centered on each of the 25 crystals computed by the use of standard (left) and optimized (right)

6.7 Energy resolution as a function of energy measured with an optimal set of weights in the crystal arrays $3 \times 3$ with the beam centered of each of 25 crystal arrays on the supermodule SM16 during the testbeam campaign 2006 [6] . . . . . . . . . 152

6.8 Noise contribution to the $H \rightarrow \gamma \gamma$ mass resolution for $m_{H}=100 \mathrm{GeV}$ [1] . . . . . 153

6.9 Reconstructed amplitude in ECAL barrel channels in the absence of a signal, without pile-up (red dashed distribution) and with pile-up (black distribution). A Gaussian of width of $40 \mathrm{MeV}$, representing the electronic noise, is superposed on the dashed distribution $[5] \ldots \ldots$. . . . . . . . . . . . . . . . 154

6.10 Top left: Low frequency noise $\sigma_{L F}=0.84 \mathrm{ADC}$ counts as a standard deviation of the distribution of the mean sample values of each event (150 entries). Top right: High frequency noise $\sigma_{H F}=0.79 \mathrm{ADC}$ counts as a standard deviation of the distribution of the difference of each sample value and the mean sample value over the 10 samples in the event (1500 entries). Bottom left: Total noise $\sigma_{T o t}$ $=1.16 \mathrm{ADC}$ counts as a standard deviation of the distribution of the all time sample values of all the events $(10 \times 150=1500$ entries $) . \ldots \ldots$. . . . . . .

6.11 Top figures: Total noise in ADC counts as a function of channel number for the three gains 12, 6, and 1 for the barrel supermodule SM17 (left) and the endcap 500 crystal array (right). Bottom figures: Distribution of the total noise in ADC counts for the three gains for all the SM17 channels and 500 channels of the endcap. 157

6.12 Left: Mean pedestal value as a function of the time sample for all the events. Right: Mean pedestal value over all events as a function of time sample. Both figures are for gain 12, channel 248, supermodule SM17 and show normal behaviour.157

6.13 Distribution of the total, low and high frequency noise for gain 12 measured for all the 1700 channels of the supermodule SM17 (left) and 500 endcap crystal array (right) . . . . . . . . . . . . . . . . . . . . . . 158

6.14 Total, low and high frequency noise for three gains 12,6, and 1 as a function of channel number measured on the supermodule SM17 (left) and on the 500 endcap crystal array (right). . . . . . . . . . . . . . . . . . . 159

6.15 Distributions of the total noise in ADC counts in the sum of 25 (left) and 9 (right) over all channels in supermodule SM17 measured in gain 12 . . . . . . . . . . 160

6.16 Noise correlation matrix showing the correlation of the noise between samples as a mean value over all channels of the barrel supermodule SM17 (top left) and the endcap 500 crystal arrays (top right). The bottom figures represent the corresponding autocorrelation function for the barrel as well as the endcap. The lines are only to guide the eye. . . . . . . . . . . . . . . . 
6.17 The total, low and high frequency noise for all three gains as a function of time (represented by a run number and cover a period of 3 weeks) for barrel supermodule SM16 and the endcap 500 crystal array. Each point is calculated as the average over all channels and error bars corresponds to the RMS value of this average. . . . . . . . . . . . . . . . . . . 163

6.18 Energy resolution as a function of the gain ratio using test beam data with a $180 \mathrm{GeV}$ electron beam. The energy is reconstructed for a $3 \times 3$ crystal array [11]. 165

6.19 Profile of the signal pulse for a crystal exposed to an electron beam of $180 \mathrm{GeV}$, before (left) and after (right) applying the gain ration multiplication. Samples which are recorded in highest gain 12 (red) and in middle gain 6 (blue) are shown $[13] \ldots \ldots \ldots \ldots \ldots \ldots \ldots \ldots$

6.20 Mean value of the the reconstructed amplitude for low gain 1 (top) and middle gain 6 (bottom) measured for one VFE card (5 channels) in free and fixed gain modes. Errors bars indicate the RMS value of the computed amplitudes. Measurements were performed on the VFE calibration bench at IPN Lyon. . . . 167

6.21 Distribution of the relative difference between the gain ratio $12 / 6$ computed using the weights method and analytic fit method. The gain ratios were measured by the test pulse $(4.1 \mathrm{pC})$ on the supermodule SM17 at CERN . . . . . . . . . . . 168

6.22 Relative difference of the signal pulse profiles determined in gain 12 and gain 6 . The profiles were built for the crystal 248 (supermodule SM6) using electron data. 169

6.23 Distribution of the relative difference between the gain ratio 12/6 computed using weights determined in gain 12 (weights12) and in gain 6 (weights6). The gain ratios were measured using the electron beam for $9 \times 9$ crystal array on the supermodule SM6 at CERN. . . . . . . . . . . . . . . . . . .

6.24 Distribution of the relative difference between the gain ratio $12 / 6$ computed using the weights method with $3+7$ weights (special) and with the $3+5$ weights method (standard). The gain ratios were measured using the electron beam for $9 \times 9$ crystal array on the supermodule SM6 at CERN. . . . . . . . . . . . . . . . . . 170

6.25 Distribution of the gain ratio $12 / 6$ measured using the test pulse on $(4.1 \mathrm{pC})$ the fully equipped supermodule SM17 at CERN (blue) and on the VFE calibration

bench at IPN Lyon (green). . . . . . . . . . . . . . . . . . . . . sured by the test pulse on the supermodule SM17 at CERN and on the VFE calibration bench at IPN Lyon. . . . . . . . . . . . . . . . . . . . 171

6.27 Dependence of the mean gain ratio 12/6 on the injected MGPA test pulse charge measured by the test pulse method on the supermodules SM5 and SM17. . . . . 172

6.28 Distribution of the gain ratio $12 / 6$ measured using the test pulse $(4.1 \mathrm{pC})$ and laser methods on the fully equipped supermodule SM6 (1,700 channels) at CERN. 173

6.29 Correlation (top) and relative difference (bottom) between gain ratio 12/6 measured by the test pulse and laser methods on the supermodule SM6 (1,700 channels) at CERN. . . . . . . . . . . . . . . . . . . . 173

6.30 Top: Distribution of the reconstructed amplitude in ADC counts as a function of the impact position on the crystal in the Y coordinate for crystal 253, supermodule SM22. The region selected for gain ratio measurement is also indicated. Bottom: Pulse profile in gain 12 (crystal 253, SM22) used for determination of the set of weights for signal amplitude reconstruction. . . . . . . . . . . . 
6.31 Distributions of the reconstructed amplitude over all events for crystal 253, SM22 and three gains. Data were taken in three independent runs and the hodoscope cut of $9 \times 9 \mathrm{~mm}^{2}$ was applied. The figure shows the fit using the Crystal Ball function (see Eqv. 6.10) for finding the maximum amplitude value. Meaning of the fit parameters is: $\mathrm{p} 0$ is a normalization factor, $\mathrm{p} 1, \mathrm{p} 2$ are mean and standard deviation of the Gaussian part, and p3, p4 describe the power law tail. . . . . . 176

6.32 Distributions of the gain ratio 12/6 measured using beam (green distribution) and the test pulse methods (blue distribution) for all measured crystals on the supermodules SM6 and SM22 with beam during the test beam campaign 2006 at CERN . . . . . . . . . . . . . . . . . . . . . . . . 177

6.33 Correlation (top) and relative difference (bottom) between the gain ratio $12 / 6$ measured by the test pulse and with an electron beam of $120 \mathrm{GeV}$ at CERN for 179 crystals on the supermodules SM6 and SM22. . . . . . . . . . . . 17

6.34 The normalized gain ratio $12 / 6$ as a function of the beam energy measured for 15 crystals on the supermodule SM6 et CERN . . . . . . . . . . . . . . . . . 179

6.35 Correlation between gain ratio $12 / 6$ measured by the test pulse and with an electron beam for 6 different energies (from 30 to $150 \mathrm{GeV}$ ) on the supermodule SM6 at CERN. The black diagonal lines are only to guide the eye. . . . . . . . . 179

6.36 Top: The 12/6 gain ratio for a typical crystal as a function of run number measured on the supermodule SM17 over a period of 3 weeks during the test beam campaign. Bottom: the RMS of the distribution of $12 / 6$ gain ratio values for all runs measured on SM17, for each crystal. . . . . . . . . . . . . . . . . . 181

6.37 Global distribution for all crystals and all runs, of the values of RMS divided by fitted mean, of the gain 12 amplitudes (SM17), used for the data cleaning procedure.182

6.38 Energy resolution as a function of energy for the uniform impact measured on the supermodule SM6 using a $3 \times 3$ crystals array centered on the crystal 248 (left) and for 25 crystals (right). [17] . . . . . . . . . . . . . . . 183 



\section{List of Tables}

1.1 The main parameters of the collider relevant for the LHC detectors. . . . . . . . 38

1.2 Main parameters of the CMS solenoid $[8] \ldots \ldots \ldots \ldots$. . . . . . . . 47

2.1 The targeted contributions to the energy resolution of the CMS ECAL in barrel and endcap for the $5 \times 5$ crystal array at low (high) luminosity. . . . . . . . 57

2.2 Basic properties of the $\mathrm{PbWO}_{4}$ scintillating crystals of the CMS ECAL $[1,11] \ldots \ldots \ldots \ldots \ldots \ldots$

2.3 Main properties of the Avalanche Photodiode developed for the CMS ECAL [19,20]. 63

2.4 Main properties of the Vacuum Phototriodes developed for the CMS ECAL [5,

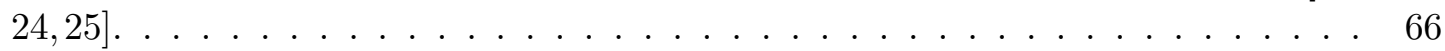

3.1 Fast control encoding of the synchronous broadcast signals. . . . . . . . . . . . 82

4.1 Multi gain pre-amplifier (MGPA) specification [5] . . . . . . . . . . . . . 95

4.2 MGPA noise expressed as ENC (Equivalent Noise Charge) in electrons measured for all three gains with input capacitances $C_{i n}=200 \mathrm{pF}$ and $C_{i n}=50 \mathrm{pF}$ for the barrel and endcap, respectively $[5] \ldots \ldots \ldots \ldots$. . . . . . . . . .

4.3 Valid output bus configurations of the AD41240. The DDR indicated "Double Data Rate" regime, when output clock runs at $40 \mathrm{MHz}$, but data are available on both edges of the clock. . . . . . . . . . . . . . . . . . . 102

4.4 Summary of the AD41240 specification parameters extracted from the device characterization $[10] \ldots \ldots \ldots \ldots$. . . . . . . . . . . . . 106

4.5 LVDS_RX performance specifications. . . . . . . . . . . . . 107

5.1 Pulse height and pedestal values for all tested VFE barrel cards which fulfill specifications measured for the free gains 1,6 , and $12 \ldots \ldots 113$

5.2 The mean values of the power supply: analog, digital, buffers voltages and currents measured for all 12,887 tested barrel VFE cards. . . . . . . . . . . . . . . 115

5.3 Expected (measured) values of the analog, digital and buffers currents for each individual component and for a fully functional barrel VFE card. Measured values are the mean values over all the VFE cards measured in power-on test. . . . . . . 115

5.4 The specification values for VFE cards. The VFE card with measured values outside of these specification limits was considered as bad and rejected from the production queue. The slope and offset are related to the linear fit (see Par. Calibration curve and Fig. 5.10) of the VFE response versus injected charge. . . . 122

5.5 Experimental values of the injected charges in $\mathrm{pC}$ for the barrel and endcap VFE cards for gains 1,6 , and $12 . \ldots \ldots \ldots \ldots \ldots$ 
5.6 Mean value of the slopes (ADC count $/ \mathrm{pC}$ ) and offsets (ADC count) of the injected charges versus pulse height and its dispersions (\%) for all the barrel and endcap VFE cards for three gains 1,6 , and $12 \ldots \ldots \ldots \ldots \ldots$

5.7 Dispersions of the slopes for gain 1, 6, and 12 before and after the test board and time intercalibration. . . . . . . . . . . . . . . . . . . 128

5.8 Mean values of the gain ratios and corresponding dispersions for all the barrel and endcap VFE cards. . . . . . . . . . . . . . . . . . . 130

5.9 Non-linearity of all the barrel and endcap VFE cards measured over a full dynamic range for all three gains. . . . . . . . . . . . . . . . . . . . . . 133

6.1 Electronic noise in ADC counts measured in a single channel and arrays of $3 \times 3$ and $5 \times 5$ crystals as an average value over the entire supermodule SM17 and the endcap 500 crystal array for a given pedestal run. $n+m$ describes the weights method configuration, where $n$ is the number of weights for pre-samples and $m$ is a number of weights applied to the peak samples. . . . . . . . . . . . . 160

6.2 Average values of the total, low and high frequency noise measured in all three gains on the supermodule SM16 and the endcap 500 crystal array. The average noise was calculated over the whole studied period, see text. . . . . . . . . . . . 164

6.3 Summary results of the gain ratio $12 / 6$ determined with beam and by the test pulse $(4 \mathrm{pC})$ on the four studied supermodules at CERN H4. On SM22 data were also taken in gain 1 , so the results for the gain ratio $12 / 1$ are also included. . . . 178 


\section{List of Acronyms}

AB Algorithm Board

ACCOS Automatic Crystal COntrol System

ADC Analogue-to-Digital Convertor

ALICE A Large Ion Collider Experiment

AOI Automatic Optical Inspection

APD Avalanche Photodiode

ASIC Application Specific Integrated Circuit

ATLAS A Toroidal LHC ApparatuS

BELLE B detector at KEK in Japan

BTCP Bogorodisk Techno-Chemical Plant

BaBar B and B-Bar Experiment

C4I Centre de Compétences en Conception de Circuits Intégrés

CCS Control and Clock System

CCU Communication and Control Unit

CERN European Organization for Nuclear Research (Organisation européenne pour la recherche nucléaire)

CMS Compact Muon Solenoid

CRC Cyclic Redundancy Check

CRISTAL Cooperating Repositories and Information System for Tracking Assembly Lifecycles

CSCs Cathode Strip Chambers

DAC Digital-to-Analogue Converter

DAQ Data Acquisition

DCC Data Concentrator Card 
DCS Detector Control System

DCU Detector Control Unit

DDR Double Data Rate

DNL Differential Non Linearity

DOH Digital OptoHybrid

DSP Detector Signal Processing

DSP Digital Signal Processing

DTs Drift Tubes

ECAL Electromagnetic Calorimeter

ECC Error Correction Code

ENC Equivalent Noise Charge

ENOB Effective Number of Bits

FENIX Front-End New Intermediate data eXtractor

FFT Fast Fourier Transform

FGV Fine Grain Veto

FIFO First In, First Out

FPPA Floating Point Pre-Amplifier

GCT Global Calorimeter Trigger

GOH Gigabit Optical Hybrid

GOL Giga-bit Optical Link

GTP Global Trigger Processor

GT Global Trigger

HCAL Hadron Calorimeter

HV High Voltage

KEK High Energy Accelerator Research Organization (Kō Enerugì Kasokuki Kenkyū Kikō)

LEP Large Electron Positron Collider

LHC Large Hadron Collider

LHCb Large Hadron Collider - Beauty

LSB Least Significant Bit 
LVDS Low-Voltage Differential Signaling

LVD Low Voltage Distribution

LVPS Low Voltage Power Supplies

LVR Low Voltage Regulator

LV Low Voltage

MEM Monitoring Electronic Module

MGPA Multi-Gain Pre-Amplifier

PCB Printed Circuit Board

PLL Phase Locked Loop

POTB Power On Test Board

PSI Paul Scherrer Institut

PSRR Power Supply Rejection Ratio

QA Quality Assurance

QCD Quantum Cromodynamics

QPLL Crystal Oscillator Phase Locked Loop

RB Receiver Block

RCT Regional Calorimeter Trigger

REDACLE Relational Ecal Database for Construction Level

RIE Research Institute Electron

RPCs Resistive Plate Chambers

SEU Single Event Up-set

SFDR Spurious-Free Dynamic Range

SIC Shanghai Institute of Ceramics

SINAD Signal-to-Noise and Distortion Ratio

SLAC Stanford Linear Accelerator Center

SLB Synchronization and Link Board

SM Standard Model

SNR Signal-to-Noise Ratio

SPS Super Proton Synchotron 
SRF Selective Readout Flag

SRP Selective Readout Processor

TAX Target Attenuator eXperimental areas

TCC Trigger Concentrator Card

TCP Trigger Cell Processor

TDC Time to Digital Converter

THD Total Harmonic Distortion

TRB Token Ring Board

TTC Timing Trigger and Control system

TTS Trigger Throttling System

VFE Very-Front-End

VPT Vacuum Phototriode

mFEC mezzanine Frond-End Controller 


\section{Introduction}

Experiments at the Large Hadron Collider (LHC) will study proton-proton collisions at a centerof-mass energy of $14 \mathrm{TeV}$, at a maximum design luminosity of $10^{34} \mathrm{~cm}^{-2} \mathrm{~s}^{-1}$ and a crossing rate of $40 \mathrm{MHz}$. One of the main experiments is the Compact Muon Solenoid (CMS) [1], which is a general-purpose detector designed to measure the position, energy and momentum of photons, electrons, muons and other charged particles with high precision. The CMS detector which has an overall length of $21.6 \mathrm{~m}$, with a calorimeter coverage to a pseudo-rapidity ${ }^{1}$ of $|\eta|=5\left(\theta \approx 0.8^{\circ}\right)$, a radius of $7.5 \mathrm{~m}$ and a total weight of about $12,500 \mathrm{t}$. The CMS experiment consists of a powerful inner tracking system based on fine-grained micro-strip and pixel detectors, a scintillating crystal calorimeter followed by a sampling hadron calorimeter made of plastic scintillator tiles inserted between copper absorber plates, and a high-magnetic-field (4T) superconducting solenoid surrounded by a multilayer muon system installed in its yoke.

One of the main physics goals pursued will be the search for the Higgs boson, which is the missing corner-stone in the Standard Model of particle physics. In the mass range of 110 - $150 \mathrm{GeV}$ the favored process for Higgs boson detection is via its decay into two photons, whereas the processes $H \rightarrow W W$ and $Z Z$ will be important at higher masses, where the $W$ and $Z$ bosons are identified via their leptonic decays. It becomes clear that an excellent energy and angular resolution for the detection of electrons and photons is mandatory and therefore a central design feature of the CMS is a fully active very high performance electromagnetic calorimeter. A scintillating crystal calorimeter offers the best performance for energy resolution since most of the energy from electrons or photons is deposited within the homogeneous active crystal volume of the calorimeter. High density crystals with a small Molière radius allow a very compact electromagnetic calorimeter system.

Several large crystal calorimeters are successfully operating in high-energy physics experiments, e.g. BaBar [2] at SLAC and BELLE [3] at KEK with 6,780 and 8,736 CsI(Tl) crystals, respectively. However, these detectors do not face the difficult experimental environment at the LHC which imposes stringent and challenging requirements on the detector specifications: under nominal LHC operation, every $25 \mathrm{~ns}$ an average of 20 events with some 1000 charged tracks will be produced. Compared with mentioned experiments, where high precision and wide dynamic range are required, the same criteria have to be met but at a much higher speed and in a much more hostile radiation environment (a radiation dose rate of $\sim 1-2 \mathrm{kGy} /$ year at maximum operation luminosity) at the LHC.

The concept of calorimeter read-out electronics is also challenging which must be very fast to match $25 \mathrm{~ns}$ LHC bunch crossing rate and provide energy measurement with high precision over a large dynamic range from about $50 \mathrm{MeV}$ to several $\mathrm{TeV}$ corresponding to almost 16 bit

\footnotetext{
${ }^{1}$ Pseudo-rapidity $\eta$ is a function of the production angle $\theta$ with respect to the beam axis. It is defined as $\eta=-\ln \left[\tan \left(\frac{1}{2} \theta\right)\right]$.
} 
accuracy in digitization. At the same time, it must withstand the severe working conditions during the 10-year experiment life-time. That is why, all the parts of the frontal electronics, including so called Very-Front-End (VFE) cards that are the main subject of this work, need to pass extensive test and calibration programs to guarantee their reliability and elaborate working performance.

The dissertation is organized in six chapters. In the first one, a short overview of the LHC accelerator complex and its main physical potential is given. In the second part of this chapter the CSM experiment is briefly introduced. The second chapter describes the calorimeter design, its instrumentation and working principles of the most important parts and systems as well as the basic production tests of single components and their final status. In the third chapter, the calorimeter electronics system, including both on-detector and off-detector systems, are presented. The following chapter, chapter four, is focused on detailed description of the VFE electronics and properties of its individual components. The calibration procedure of the VFE cards are given in chapter five where the most relevant calibration results are also summarized. Finally, the performance of the readout electronics evaluated during test beam experiments at CERN and results obtained are given in chapter six. 


\section{Bibliography}

[1] CMS Collaboration, The Compact Muon Solenoid - Technical Proposal, ser. Technical Design Report CMS. Geneva: CERN, 1994, no. CERN/LHCC 94-38.

[2] BABAR Collaboration, "BABAR detector - technical design report," SLAC, http://www.slac.stanford.edu/BFROOT/www/doc/TDR/, Tech. Rep., Mar 1995.

[3] K. Miyabayashi, "BELLE electromagnetic calorimeter," Nucl. Instrum. Methods Phys. Res., A, vol. 494, pp. 298-302, 2002. 



\section{Chapter 1}

\section{Large Hadron Collider and CMS experiment}

\subsection{Introduction}

The Standard Model (SM) ${ }^{1}$ is a quantum field theory which includes our present knowledge of basic constituents of the matter and their interactions. The model has been extensively tested during the past several decades and no disagreement was found between theoretical predictions and experimental measurements. However, the last fundamental particle predicted by SM, so-called the Higgs boson, that plays a crucial role in the spontaneous electroweak symmetry breaking mechanism by which particles acquire mass, has not been observed yet. Therefore, a discovery of the Higgs particle (or a clear statement on its non existence), whose mass is unknown but which should not exceed $\sim 1 \mathrm{TeV}$, becomes a prime motivation for the Large Hadron Collider.

In this chapter, a short overview of the Large Hadron Collider and its physics potential are outlined with respect to the CMS experiment.

\subsection{Large Hadron Collider}

The Large Hadron Collider (LHC) $[1,2]$ is a two-ring superconducting collider installed in the $27 \mathrm{~km}$ long circular tunnel previously built for the Large Electron Positron Collider (LEP) at CERN, which will provide collisions of high energy protons or heavy ions (HI). The main design parameters are a luminosity of $L_{p p}=10^{34} \mathrm{~cm}^{-2} \mathrm{~s}^{-1}$ and $L_{H I}=10^{27} \mathrm{~cm}^{-2} \mathrm{~s}^{-1}$, centre-of-mass energy of $\sqrt{s_{p p}}=14 \mathrm{TeV}$ and $\sqrt{s_{H I}}=5.52 \mathrm{TeV}$ per nucleon and bunch crossing interval of $25 \mathrm{~ns}$ and $100 \mathrm{~ns}$ for protons and heavy ions collisions, respectively. At nominal luminosity about 20 collisions occur per bunch crossing. The other machine parameters relevant to the LHC detectors are summarized in Tab.1.1. In the following text only proton-proton regime is considered.

A maximum designed beam energy of $7 \mathrm{TeV}$ is derived from the maximum magnetic induction that must be achieved in order to keep accelerated particles on the orbit. This results in a

\footnotetext{
${ }^{1}$ The Standard Model is a gauge theory based on the $S U(3)_{C} \times S U(2)_{T} \times U(1)_{Y}$ symmetry group, where the $C$ is color and $T, Y$ denote week isospin and hypercharge, respectively. The $S U(3)_{C}$ symmetry of Quantum Cromodynamics (QCD) describing the strong interaction of quarks mediated by eight massless gluons. The $S U(2)_{T} \times U(1)_{Y}$ is the symmetry group related to the electroweak interaction with four associated bosons: $\gamma, Z^{0}, W^{ \pm}$.
} 


\begin{tabular}{|ll|c|c|}
\hline \hline Parameter & & $\mathbf{p}-\mathbf{p}$ & $\mathbf{P b}-\mathbf{P b}$ \\
\hline Energy per nucleon & $E(\mathrm{TeV})$ & 7 & 2.76 \\
Dipole field at 7 TeV & $B(\mathrm{~T})$ & 8.33 & 8.33 \\
Design luminosity & $L\left(\mathrm{~cm}^{-2} \mathrm{~s}^{-1}\right)$ & $10^{34}$ & $10^{27}$ \\
Bunch separation & $(\mathrm{ns})$ & 25 & 100 \\
Number of bunches & $k_{B}$ & 2808 & 592 \\
Number of particles per bunch & $N_{p}$ & $1.15 \times 10^{11}$ & $7.0 \times 10^{7}$ \\
$\beta$-value at interaction point & $\beta^{*}(\mathrm{~m})$ & 0.55 & 0.5 \\
RMS beam radius at interaction point & $\sigma^{*}(\mu \mathrm{m})$ & 16.7 & 15.9 \\
Luminosity lifetime & $\tau_{L}(\mathrm{hr})$ & 15 & 6 \\
Number of collision/crossing & $n_{c}$ & $\approx 20$ & - \\
Transverse emittance & $\varepsilon_{n}$ & $3.75 \mu \mathrm{m}$ & - \\
\hline \hline
\end{tabular}

Table 1.1: The main parameters of the collider relevant for the LHC detectors.

dipole magnetic field of $8.33 \mathrm{~T}$ which requires usage of superconducting magnets operating at a temperature of super-fluid helium $1.9 \mathrm{~K}$. The collider comprises 1,232 main bending dipole magnets of $15 \mathrm{~m}$ length (14.2 m magnetic length), which cover $2 / 3$ of the circumference. Many other magnetic elements such as arc and tuning quadrupoles, sextupoles, etc., are needed for optics matching and the final focus.

The event rate $R$ defined as the number of events generated per second by proton-proton collisions for a given process is proportional to the process cross-section $\sigma$ and to the luminosity $L$ :

$$
R=L \sigma .
$$

Few examples of the cross-section and event rate for a luminosity of $10^{33} \mathrm{~cm}^{-2} \mathrm{~s}^{-1}$ for several processes in proton-proton collisions as a function of the centre-of-mass energy are shown in Fig. $1.1[3]$.

The luminosity depends only on the beam parameters of the machine and for a gaussian beam profile is given as:

$$
L=\frac{\gamma f k_{B} N_{p}^{2}}{4 \pi \varepsilon_{n} \beta^{*}} F,
$$

where $\gamma$ is the Lorentz factor, $f$ is the revolution frequency $(11.245 \mathrm{kHz}), k_{B}$ is the number of the bunches, $N_{p}$ is the number of protons per bunch, $\varepsilon_{n}$ is the normalized transverse emittance with a design value of $3.75 \mu \mathrm{m}, \beta^{*}$ is the betatron function at the interaction point, and $F$ is the reduction factor due to the crossing angle. From the Eq.1.2 it is clear that the high luminosity can be obtained only if the bunch crossing frequency and the number of protons per bunch are high and the beam intersection area is small.

It is expected that the maximum design luminosity will be reached progressively in several steps. During the first year of physics running the luminosity will increase from $10^{32}$ to $2.10^{33} \mathrm{~cm}^{-2} \mathrm{~s}^{-1}$ and thus so called low luminosity phase. In this year an integrated luminosity about $5 \mathrm{fb}^{-1}$ is expected followed about $10 \mathrm{fb}^{-1}$ per year up to the next step into high luminosity regime when the maximum designed luminosity of $L=10^{34} \mathrm{~cm}^{-2} \mathrm{~s}^{-1}$ will be achieved with an integrated luminosity of $100 \mathrm{fb}^{-1}$ per year. Discovery potential for a SM Higg boson at the LHC is presented in Fig. 1.2, which shows the statistical significance in the most important discovery channels with an integrated luminosity of $30 \mathrm{fb}^{-1}$ for the Higgs boson as a function of $\mathrm{m}_{H}$ [4] in the CMS experiment. 


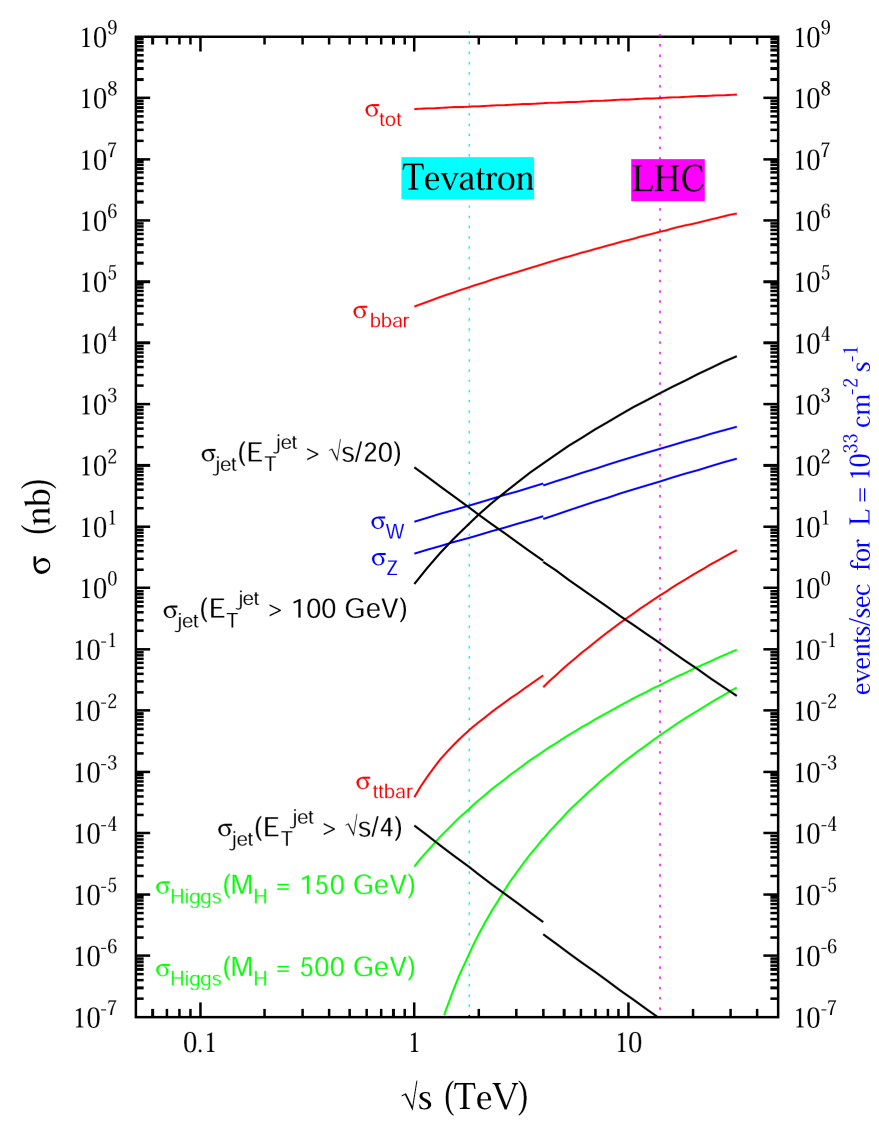

Figure 1.1: Production cross-section for various processes at hadron $\mathrm{p}-\mathrm{p}$ and $\mathrm{p}-\overline{\mathrm{p}}$ colliders as a function of the machine centre-of-mass energy [3].

\subsubsection{LHC physics potential}

One of the main aims of LHC is to search for the Higgs boson and thus investigate the nature of the electroweak symmetry breaking. For this reason, two general purpose experiments, ATLAS (A Toroidal LHC ApparatuS) and CMS, have been designed and optimized to cover the large spectrum of possible physics signatures, which are accessible at the high luminosity and centreof-mass energy of the LHC. Fig. 1.1 shows production cross-sections as a function of the centreof-mass energy for several channels at hadron colliders. One can see, for instance, that the cross-section for jets $\left(\mathrm{E}_{T}>100 \mathrm{GeV}\right)$ is five orders of magnitude larger than the cross section for a Higgs boson of mass $150 \mathrm{GeV}$ at $14 \mathrm{TeV}$. Therefore, the Higgs boson decaying into jets cannot be detected, since the final states are swamped by large QCD background, and decays to leptons and photons have to be used instead. On the other hand, the Higgs boson from jet can be detected if it is produced in association with additional particles $(t \bar{t} H, W H, Z H)$.

The current lower limit on the mass of the Higgs boson $\left(m_{H}>114.4 \mathrm{GeV}\right.$ at $95 \%$ confidence level) comes from the direct searches at the Large Electron Positron (LEP) experiments at CERN. The upper limit derived from the fit to electroweak data is $m_{H}<251 \mathrm{GeV}$ at $95 \%$ confidence level [3]. The production of the Higgs boson at LHC is expected through several mechanisms, whose cross sections as a function of the Higg mass over the range from $100 \mathrm{GeV}$ 


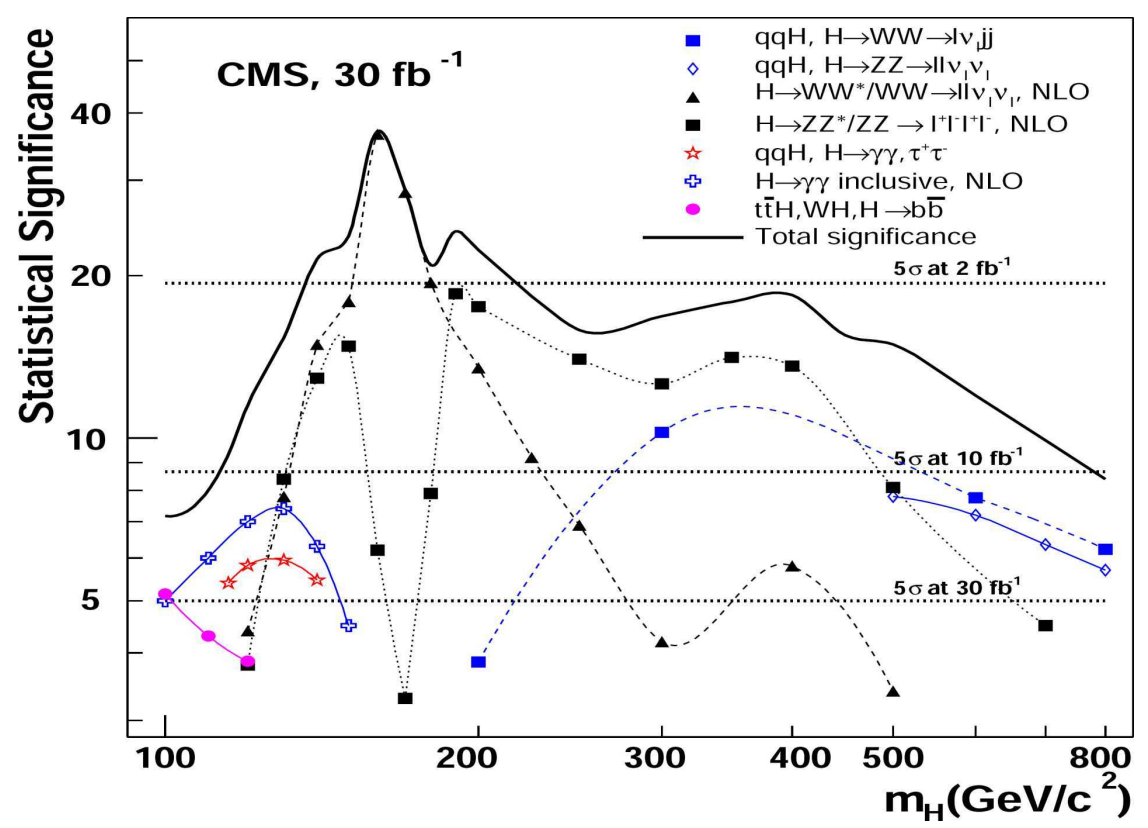

Figure 1.2: Expected statistical significance with an integrated luminosity of $30 \mathrm{fb}^{-1}$ for the SM Higgs boson as a function of $\mathrm{m}_{H}$ in the CMS experiment [4].

to $500 \mathrm{GeV}$ are shown in Fig. 1.3 [4]. It can be seen that the dominant production channel for all masses is the gluon-gluon fusion through top quark loop $g g \rightarrow H$, followed by weak gauge boson fusion process $q q \rightarrow q q H$ whose cross-section is lower by one order of magnitude. The production with association, $q \bar{q} \rightarrow H W, q \bar{q} \rightarrow H Z, g g / q \bar{q} \rightarrow t \bar{t} H$ and $g g / q \bar{q} \rightarrow b \bar{b} H$, have crosssection higher than $0.1 \mathrm{pb}$ for $m_{H}<200 \mathrm{GeV}$ [5]. The Higgs boson in these processes is search through the identification of the associated $b$ jets or reconstruction of $W / Z$ and top masses.

The branching fractions of the Higgs decays as a function of its mass is shown in Fig. 1.3 right [4]. The main channels can be classified depending on the Higgs boson mass into three regions $[5,6]$ :

- Low mass region $\left(m_{H} \leq 130 \mathrm{GeV}\right)$ - There are two decays modes which are experimentally important: $H \rightarrow b \bar{b}$ and $H \rightarrow \gamma \gamma$. The first one has a branching fraction close to one and is thus the dominant channel over all this low mass region. Unfortunately, as the signal-tonoise ratio for inclusive production is smaller than $10^{-5}$, it will be impossible to exploit this channel directly due to the large dijet background. Nevertheless, the associated production $t \bar{t} H, W H, Z H$ with an additional lepton signature where the background is significantly reduced can be used.

The $H \rightarrow \gamma \gamma$ channel has a smaller branching ratio $\left(10^{-3}\right)$, but the signal-to-noise ratio is much more favorable than for $H \rightarrow b \bar{b}$ channel. The backgrounds come mainly from irreducible $g g \rightarrow \gamma \gamma$ production and from jet-jet production, where one or both jets fake a photon. In order to extract a narrow peak of $\gamma \gamma$ invariant mass, high requirements are put on the performance of the electromagnetic calorimeter in term of energy end direction resolution. 

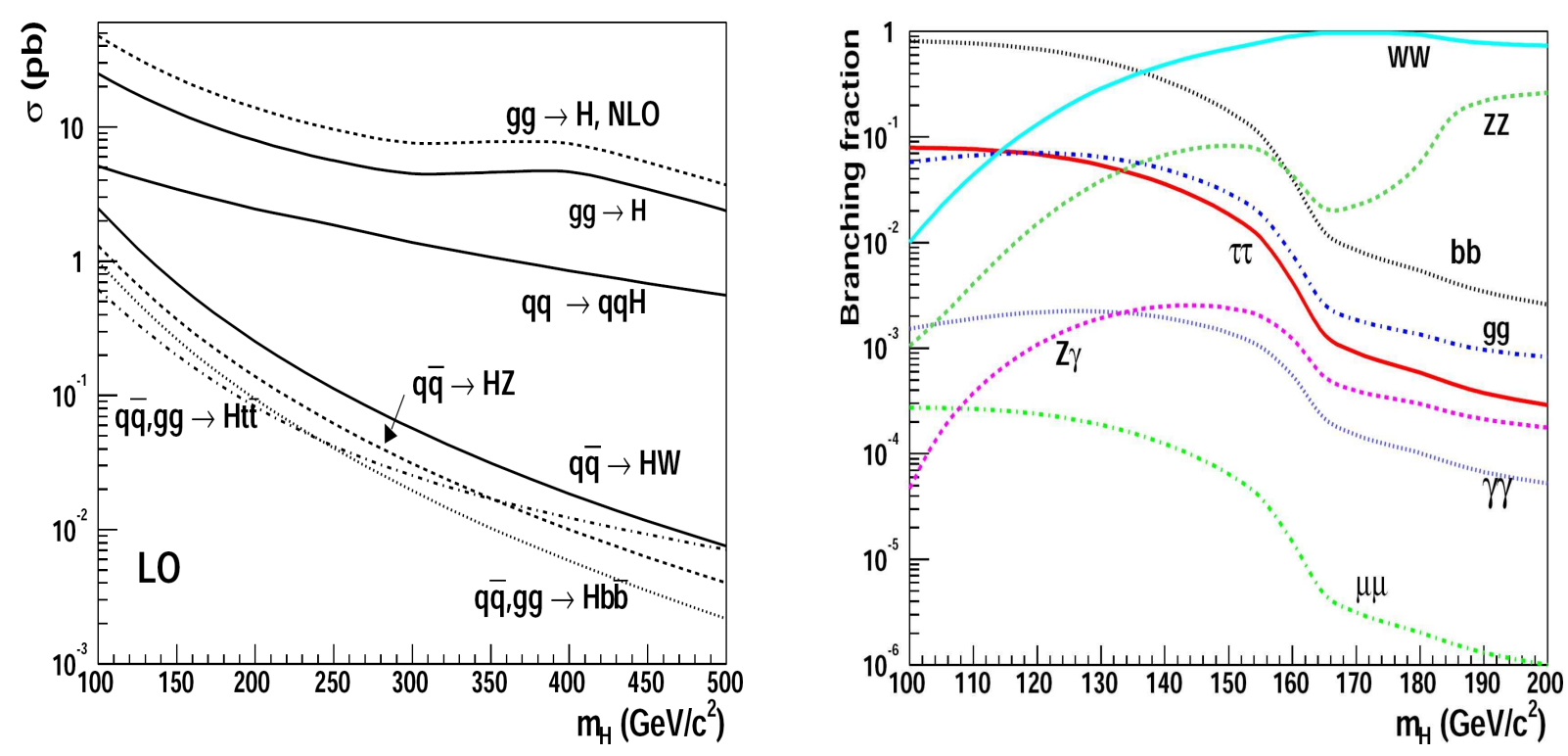

Figure 1.3: Left: The leading order cross section for the SM Higgs boson as a function of $\mathrm{m}_{H}$. Right: Branching fractions for the SM Higgs boson as a function of $\mathrm{m}_{H}$ [4].

- Intermediate mass region $\left(130 \mathrm{GeV}<m_{H}<2 m_{Z}\right)$ - The most promising channels in this region are $H \rightarrow W W^{*} \rightarrow \ell \nu \ell \nu$ and $H \rightarrow Z Z^{*} \rightarrow 4 \ell$, where one of the vector bosons can be virtual. The $Z Z$ fully leptonic decay has a very clean experimental signature. The $W W$ decay is the best candidate for the Higgs search in the mass range $150-190 \mathrm{GeV}$ due to the reduction of the branching ratio for $Z Z$ close to the $170 \mathrm{GeV}$.

- High mass region $\left(m_{H} \geq 2 m_{Z}\right)$ - In this region, the most promising channel is $H \rightarrow Z Z \rightarrow$ $4 \ell$. The irreducible background is smaller than in the intermediate region. In addition, the intristic Higgs width is larger than experimental mass resolution and therefore the detector performance is less critical. For very large masses $\left(m_{H}>700 \mathrm{GeV}\right)$, the production crosssection decreases fast, the Higgs width increases, and the branching ratio becomes too small. Therefore, other channels with higher branching ratio are used: $H \rightarrow Z Z \rightarrow \ell \ell \mu \mu$ or $H \rightarrow W W \rightarrow \ell \mu \jmath \jmath$.

\subsubsection{Detectors at $\mathrm{LHC}$}

The four main experiments will operate on the LHC: the Compact Muon Solenoid (CMS) [7,8], A Toroidal LHC ApparatuS (ATLAS) [9,10], LHC-beauty (LHCb) [11] and A Large Ion Collider Experiment (ALICE) [12]. CMS and ATLAS are general purpose experiments to investigate a wide range of physics, including the search for the Higgs boson as well as supersymmetric particles. Generally, they have the same scientific program, but they use different technical solutions and design of detector systems. The $\mathrm{LHCb}$ detector is designed to study $\mathrm{CP}$ violation and other rare phenomena in decays of hadrons with heavy flavours, in particularly B mesons. Instead of surrounding the entire collision point with an enclosed detector, the LHCb is a singlearm spectrometer comprising of sub-detectors to detect mainly low angle particles. The ALICE experiment is designed to study the physics of strongly interacting particles and the quark-gluon 
plasma in nucleus-nucleus collisions.

\subsection{CMS experiment}

The CMS experiment allows efficient and precise measurements of neutral and charged particles generated in proton collisions over all energy range and luminosities. The CMS detector, whose schematic layout is shown in Fig. 1.4, has an overall length of $21.6 \mathrm{~m}$, a radius of $7.5 \mathrm{~m}$ and a total weight of about $12,500 \mathrm{t}$. The detector consists of several dedicated sub-detectors: a powerful tracking system, a scintillating crystal calorimeter, a sampling hadron calorimeter and a high magnetic field superconducting solenoid coupled with a multilayer muon system.

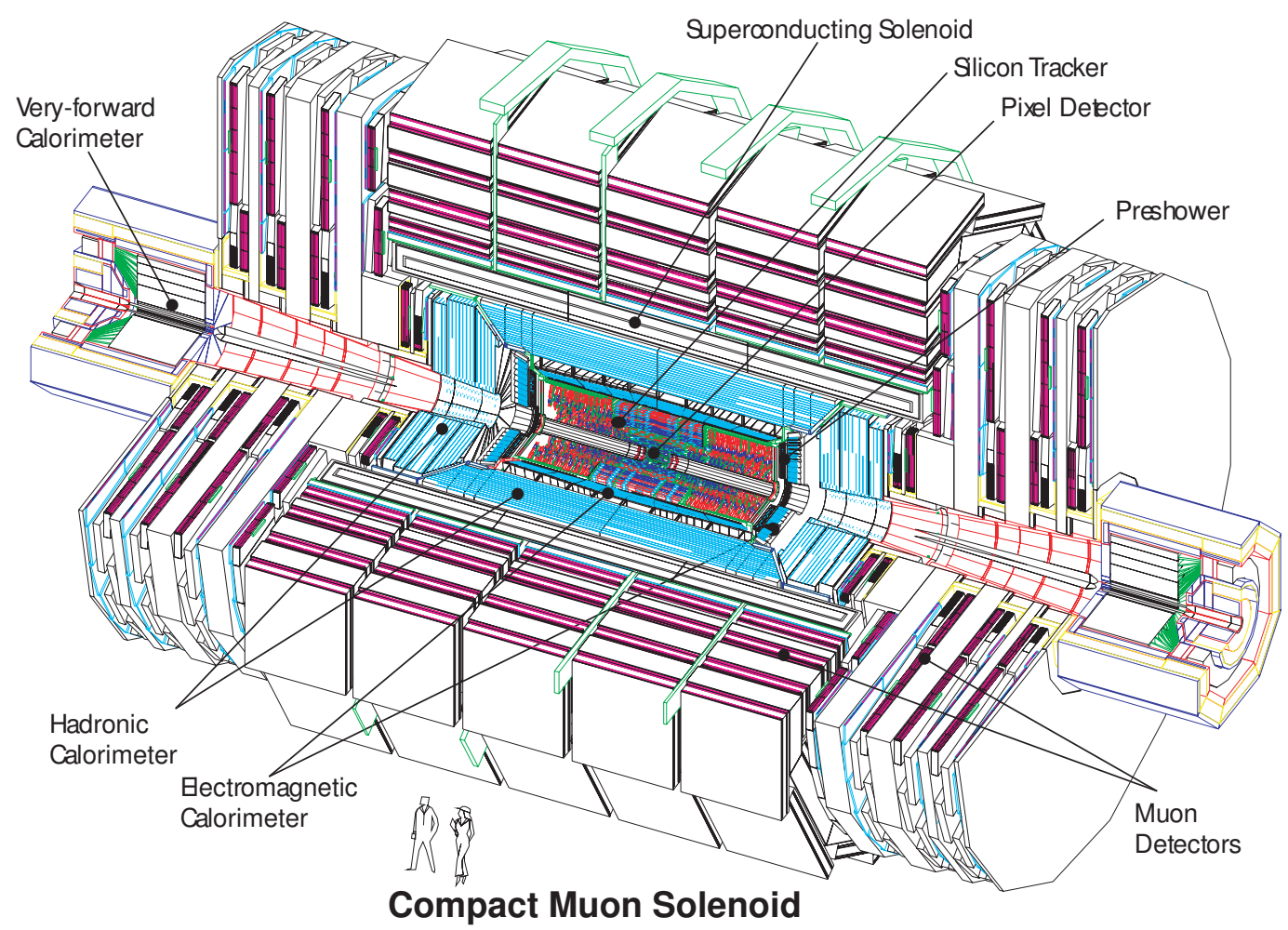

Figure 1.4: Overall view of the CMS detector.

\subsubsection{Tracker}

The tracker system measures spatial coordinates of the charged particles and, from the curvature of particle tracks due to the magnetic field, determines their momentum and identifies the interaction vertex and secondary vertices. The CMS tracker $[13,14]$ is an all silicon tracker consisting of two sub-detectors: the strip tracker and the pixel detector. In Fig. 1.5 top is shown a longitudinal view of one quadrant of the CMS tracking system configuration with respect to the collision point. 


\section{Pixel detector}

The pixel detector (see Fig. 1.6) is housed in a cylindrical volume of $1 \mathrm{~m}$ length and $30 \mathrm{~cm}$ diameter centered around the interaction point and is organized in 3 barrel layers with mean radii of $r=4.4 \mathrm{~cm}, 7.3 \mathrm{~cm}$ and $10.2 \mathrm{~cm}$, and 2 endcap disks on each side with $r=6 \mathrm{~cm}$ and $15 \mathrm{~cm}$ in radius placed at $|z|=34.5 \mathrm{~cm}$ and $46 \mathrm{~cm}$. It consists of $66.10^{6}$ pixels of $100 \mu \mathrm{m} \times 150 \mu \mathrm{m}$ in both the $(r, \phi)$ and $z$ coordinates, which are read-out by $\sim 16,000$ bump-bonded to the 768 barrel and 672 endcap pixel modules. Using analogue signal interpolation a hit resolution of $10 \mu \mathrm{m} \times 20 \mu \mathrm{m}$ in $(r, \phi)$ and $z$ can be achieved. The efficiency to find at least 3 pixels hits is $\varepsilon_{3}>90 \%$ for $|\eta|<2.2$.

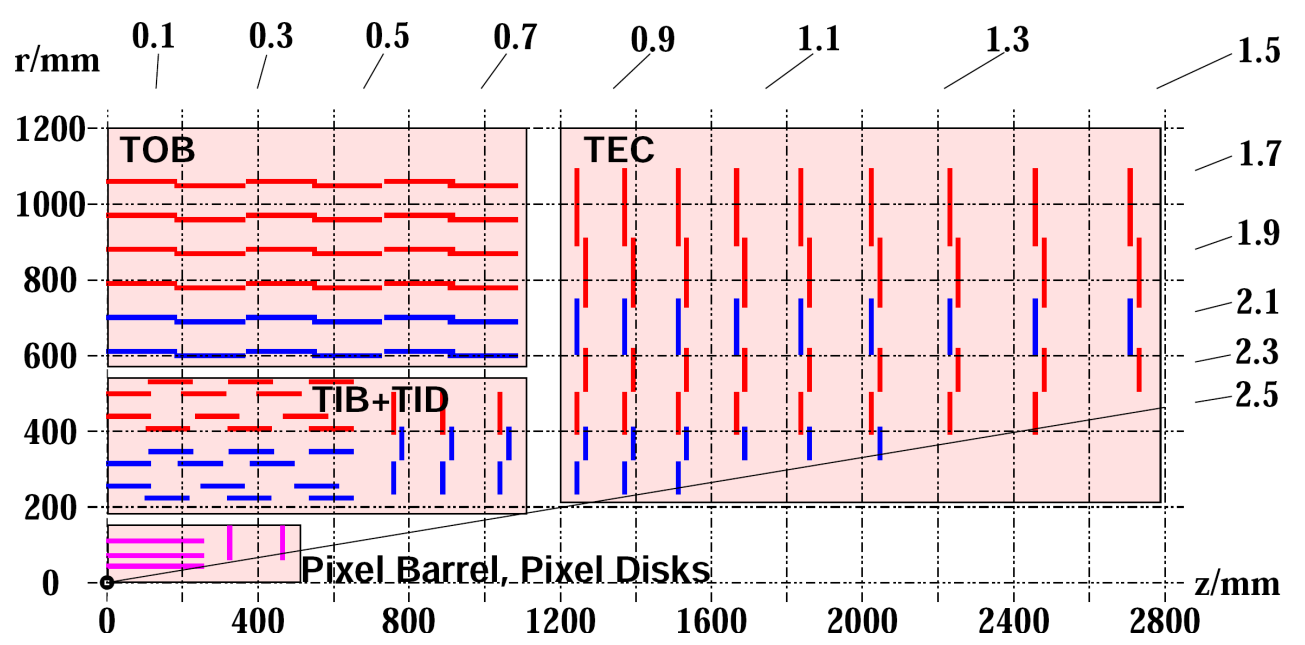

Figure 1.5: Longitudinal view of one quadrant of the CMS tracking system configuration with respect to the collision point. The TIB, TOB, TID, and TED denote the Trigger Inner and Outer Barrels, Tracker Inner Disk, and Tracker Endcap.

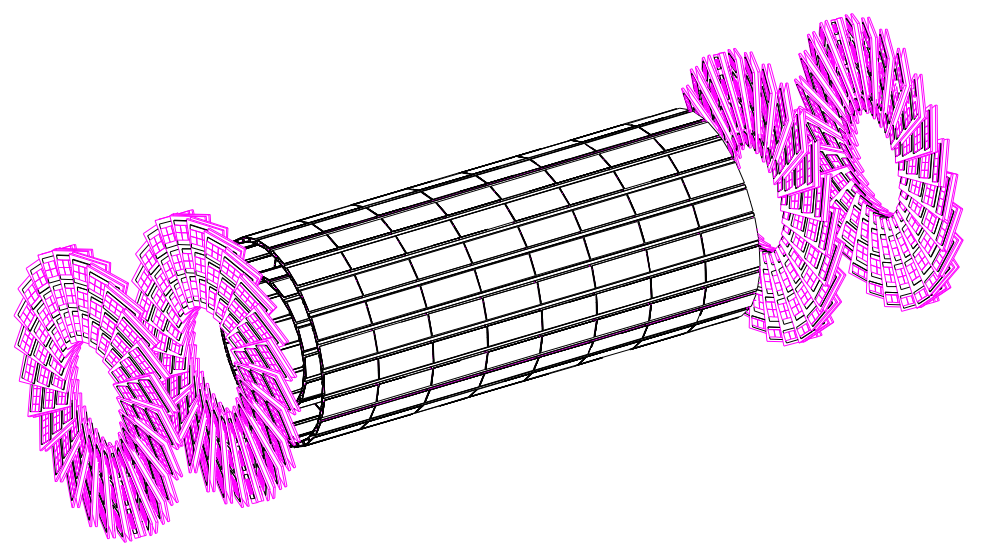

Figure 1.6: An artistic view of the pixel detector showing its barrel and endcap parts. 


\section{Strip detector}

The strip detector, which consists of 15.148 silicon strip modules, is divided into barrel and endcap regions. The barrel is composed from Tracker Inner $(|z|<65 \mathrm{~cm})$ and Outer $(|z|<$ $110 \mathrm{~cm}$ ) parts, with a strip pinch size from 80 to $120 \mu \mathrm{m}$ and from 120 to $180 \mu \mathrm{m}$, respectively. This leads in the inner part to a single point resolution between 23 and $34 \mu \mathrm{m}$ in the $(r, \phi)$ directions and $230 \mu \mathrm{m}$ in $z$, and to $35-52 \mu \mathrm{m}$ in the $(r, \phi)$ and $530 \mu \mathrm{m}$ in $z$ in outer part. The endcaps consist of the Tracker Inner Disk $(|z|<110 \mathrm{~cm})$ and Tracker Endcap $(120 \mathrm{~cm}<$ $|z|<280 \mathrm{~cm}$ ) with a pinch size from 97 to $143 \mu \mathrm{m}$ and from 96 to $183 \mu \mathrm{m}$, respectively.
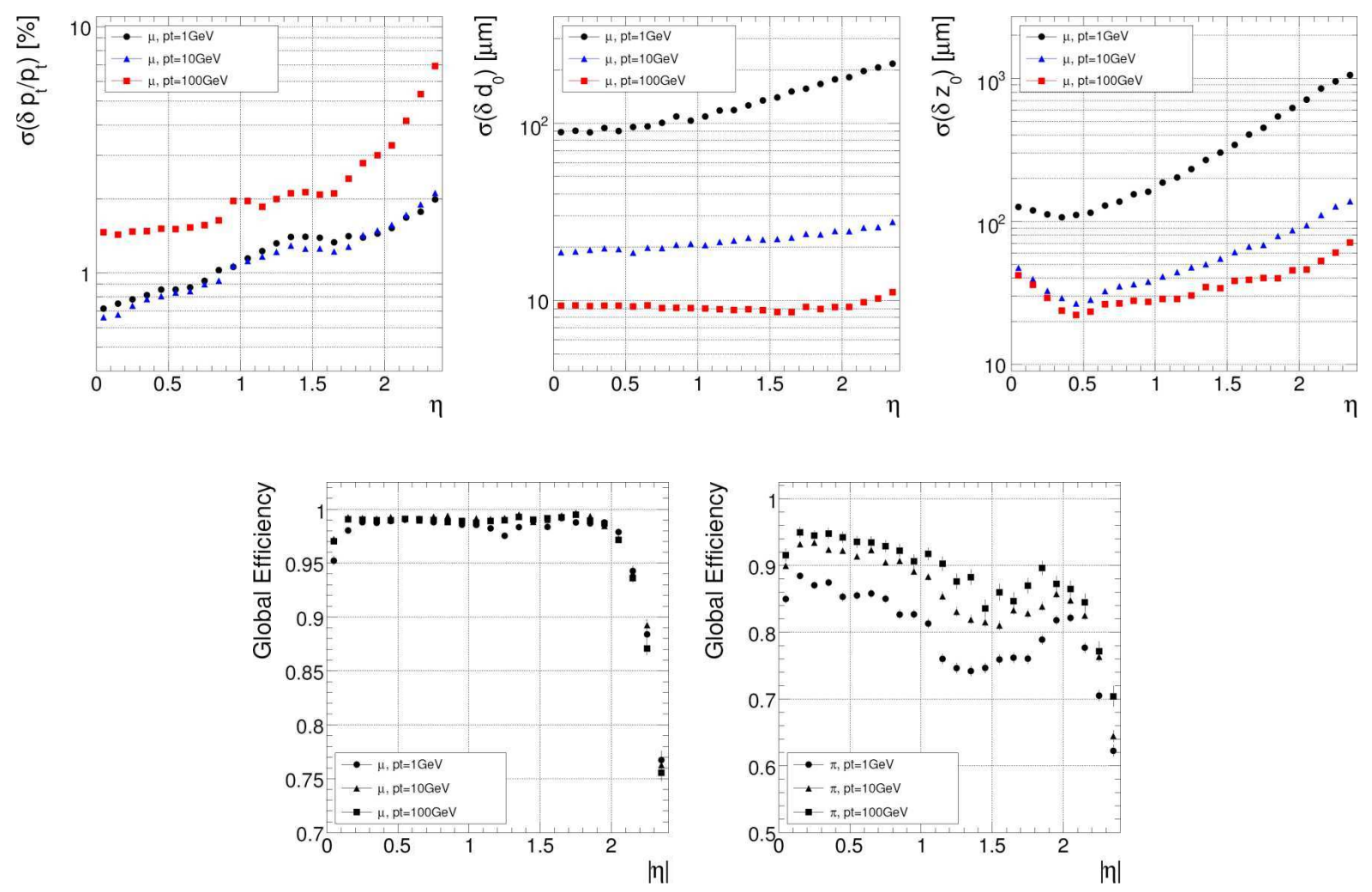

Figure 1.7: Top: Resolution of transverse momentum (left), transverse impact parameter (middle), and longitudinal impact parameter (right) for single muons with transverse momenta of 1, 10 and $100 \mathrm{GeV}$. Bottom: Global track reconstruction efficiency for muons (left) and pions (right) of transverse momenta of 1,10 and $100 \mathrm{GeV}$ [8].

\section{Tracker performance}

The performance of the tracker system (see Fig.1.7 [8]) can be demonstrated by the following parameters: The resolution of transverse momentum $\delta p_{\perp} / p_{\perp}$ and transverse and longitudinal impact parameters for a single muon with transverse momentum between 10 and $100 \mathrm{GeV} / \mathrm{c}$ are better than $4 \%, 30 \mu \mathrm{m}$, and $100 \mu \mathrm{m}$, respectively, for $|\eta|<2$. Track reconstruction efficiency for a single muons for $|\eta|<2$ are larger than $95 \%, 85 \%$ for single pions, and around $80 \%$ for pions in jets [14]. The primary vertex can be determined with $95 \%$ efficiency and, if only the pixel 
detector is used, a position resolution ranging from 20 to $70 \mu \mathrm{m}$, or down to $15 \mu$ if the whole tracker is used [15].

\subsubsection{Electromagnetic calorimeter}

The CMS electromagnetic calorimeter (ECAL) is a homogeneous compact calorimeter using radiation hard lead tungsten $\left(\mathrm{PbWO}_{4}\right)$ scintillation crystals as its active medium. It is intended for precise energy and position measurements of photons and electrons (positrons). A detailed description of ECAL is given in Chap. 2.

\subsubsection{Hadronic calorimeter}

The CMS hadronic calorimeter (HCAL) $[8,16]$ is used to measure the energy and direction of hadron jets and, due to its hermetic coverage, missing transverse energy. The HCAL also helps in the identification of electrons, photons and muons in conjunction with the tracker, electromagnetic calorimeter, and muon systems.

The HCAL is a sampling calorimeter located inside the superconducting coil. It consists of four main parts: barrel (HB), endcap (HE), outer (HO) which is outside the magnet, and forward hadron calorimeters $(\mathrm{HF})$, which provide good containment and cover a large range of pseudorapidity $|\eta|<5$ to guarantee good missing energy resolution. An arrangement of the HCAL calorimeter is depicted in Fig. 1.8 [8].

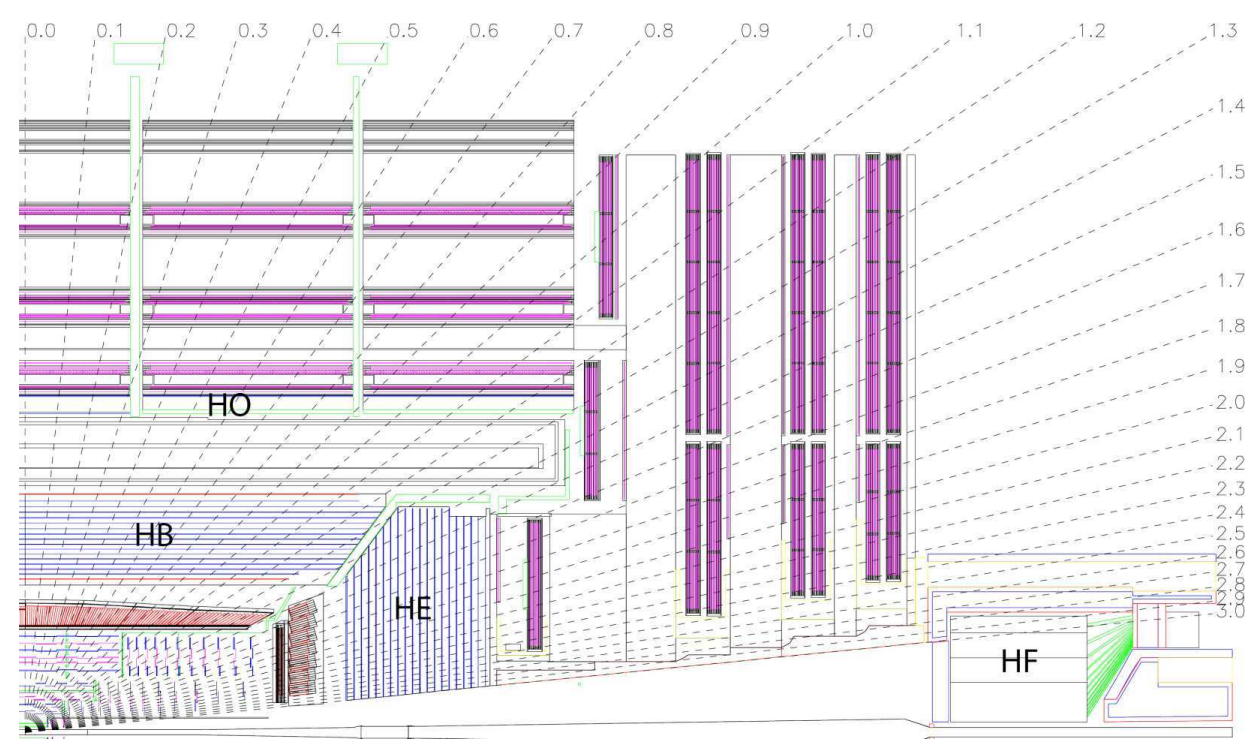

Figure 1.8: Longitudinal view of the CMS detector showing the locations of the hadron barrel (HB), endcap (HE), outer (HO) and forward (HF) calorimeters.

\section{Central hadron calorimeter}

The barrel part of HCAL covers the pseudorapidity region $|\eta|<1.4$ and consists of 2,304 towers with a segmentation of $\Delta \eta \times \Delta \phi=0.087 \times 0.087$, which match the segmentation of the ECAL 
trigger tower $(5 \times 5$ crystal array) and ensures the requiring jet separation. It has a sampling structure with brass as an absorber and plastic scintillator tiles as an active medium. Brass material was chosen because it has a short radiation length, is easy to machine and is nonmagnetic. The innermost layer of the sampling structure is made of $9 \mathrm{~mm}$ scintillators followed by 15 brass plates, each about $5 \mathrm{~cm}$ thick interleaved by $3.7 \mathrm{~mm}$ scintillators. In addition, two external stainless steel plates are added for mechanical support. The scintillator tiles are readout with wavelength-shifting fibres that are spliced to clear fibres that carry the light to the multi-channel hybrid photodiodes.

In order to increase the effective thickness of hadron calorimeter over 10 interaction lengths and thus reduce the tails in energy resolution function and improve missing transverse energy resolution, an additional scintillator (one layer in $|\eta|<1.26$ and two layers in $|\eta|<3.48$ ) is placed outside the solenoid coil that is used as an absorber with 1.4/ $\sin \theta$ interaction length. This part of HCAL is called hadron outer detector (OH).

The endcaps, which are constructed in a similar way as the barrel, are situated at each end of the barrel detector and consist of 2,304 towers. The 5 outermost towers have a segmentation of $5^{\circ}$ in $\phi$ and 0.087 in $\eta$, and the 10 innermost towers have a $\phi$ segmentation of $10^{\circ}$, while the $\eta$ segmentation varies from 0.09 to 0.35 . The endcaps cover the pseudorapidity region $1.3<|\eta|<3.0$.

\section{Forward hadron calorimeter}

In order to guarantee maximum hermeticity, the hadron forward calorimeter, which is located at $11.2 \mathrm{~m}$ from the interaction point, covers the pseudorapidity regions $3<|\eta|<5$. Due to the severe radiation conditions ( $\sim 10^{6}$ Gy after 10 years of LHC operation), the plastic scintillator is not suitable for highest rapidities and radiation hard quartz fibres were chosen as an active medium, and steel as an absorber. Thus, the calorimeter structure is composed of $5 \mathrm{~mm}$ thick steel grooved plates, where the quartz fibres are inserted in these grooves. Each forward calorimeter consist of 450 towers with a segmentation of $\Delta \eta \times \Delta \phi=0.175 \times 0.175$ (sligthly different segmentation for the lowest and highest $|\eta|$ towers). The signal originates from Čerenkov light emitted in the quartz fibres which are then readout by photomultipliers. The total depth of the absorber is $1.65 \mathrm{~m}$ that is about 10 absorption lengths.

\section{Performance of the hadron calorimeter}

The performance of HCAL is demonstrated by the jet energy resolution as a function of transverse energy for barrel, endcap and forward calorimeters are shown as Fig 1.9 [8]. The hadronic energy resolution determined combining the HCAL and ECAL barrels has a $115 \%$ stochastic term and $5.5 \%$ constant term over the beam momentum interval from 20 to $300 \mathrm{GeV} / \mathrm{c}$ [17].

\subsubsection{Superconducting solenoid}

The CMS detector uses a superconducting solenoidal magnet $[8,18]$ providing an uniform magnetic field of $4 \mathrm{~T}$. The high magnetic field is required by the muon detector in order to obtain a good momentum resolution without strict demand on the spatial resolution and the alignment of muon chambers. The main parameters of the magnet are summarized in Tab. 1.2 [8].

The magnet is composed of the superconducting coil and the magnet return yoke. The superconducting coil system consists of the coil winding constructed of four layer conductor and the ancillary subsystems required for its operation and the protection system. The conductor consists of the Rutherford superconducting cable made of NbTi strands co-extruded with high 

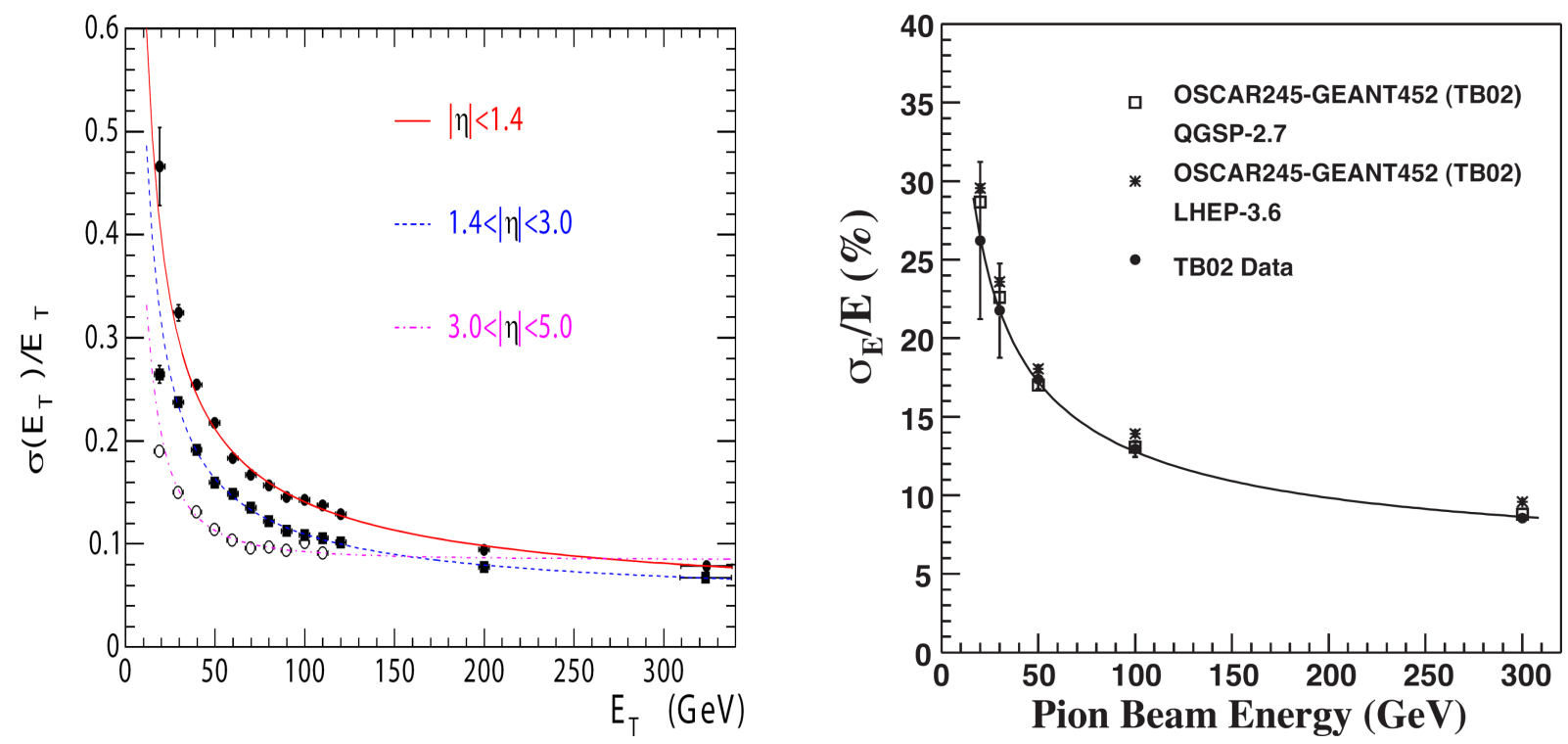

Figure 1.9: Left: The jet transverse-energy resolution as a function of the simulated jet transverse energy for barrel jets $(|\eta|<1.4)$, endcap jets $(1.4<|\eta|<3.0)$, and very forward jets $(3.0<$ $|\eta|<5.0)$. Right: Energy resolution as a function of energy for test beam pions compared to two different GEANT4 simulations.

\begin{tabular}{|l|c|}
\hline \hline Parameter & \\
\hline Magnetic field & $4 \mathrm{~T}$ \\
Inner bore & $5.9 \mathrm{~m}$ \\
Length & $12.9 \mathrm{~m}$ \\
Number of turns & 2168 \\
Current & $19.5 \mathrm{kA}$ \\
Stored energy & $2.7 \mathrm{GJ}$ \\
Magnetic radial pressure & $6.47 \mathrm{MJ}$ \\
\hline \hline
\end{tabular}

Table 1.2: Main parameters of the CMS solenoid [8]. 
purity aluminium stabilizer and external aluminium alloy reinforcing sheath. The coil sits inside a stainless steel vacuum tank and is cooled by the cryogenic system below the critical quench temperature of $4.2 \mathrm{~K}$ by means of liquid helium. The steel vessel also serves as the support structure for barrel parts of HCAL and ECAL, and the tracker.

The magnetic flux generated by the superconducting coil is returned via a $1.5 \mathrm{~m}$ thick saturated iron yoke. The yoke is comprised of 5 barrel wheels and 6 endcap disks. The wheels have a layered structure which allows the insertion of muon chambers that are also put between the 3 endcap disks. The central barrel wheel, as the only stationary part, supports the vacuum tank and the superconducting coil. The total mass of the iron in return yoke is $10,000 \mathrm{t}(6,000 \mathrm{t}$ for barrel and 2,000 $t$ for each endcap).

\subsubsection{Muon system}

The main task of the CMS Muon Detector system [19] is to efficiently identify muons and measure their momenta $\left(\Delta p_{T} / p_{T} \approx 10 \%\right)$ in the range from a few $\mathrm{GeV}$ up to several TeV. Furthermore, the muon system provides the trigger for events where muons or decay into the muons are involved, for precise time measurement of the bunch crossing. The muon detector consists of a barrel and two endcaps, which are integrated into the return yoke of the superconducting magnet. The muon detection system uses three different types of gaseous detectors: Drift Tubes (DTs), Cathode Strip Chambers (CSCs) and Resistive Plate Chambers (RPCs). Fig. 1.10 shows a layout of one quarter of the CMS muon system with deployment of its subdetectors.

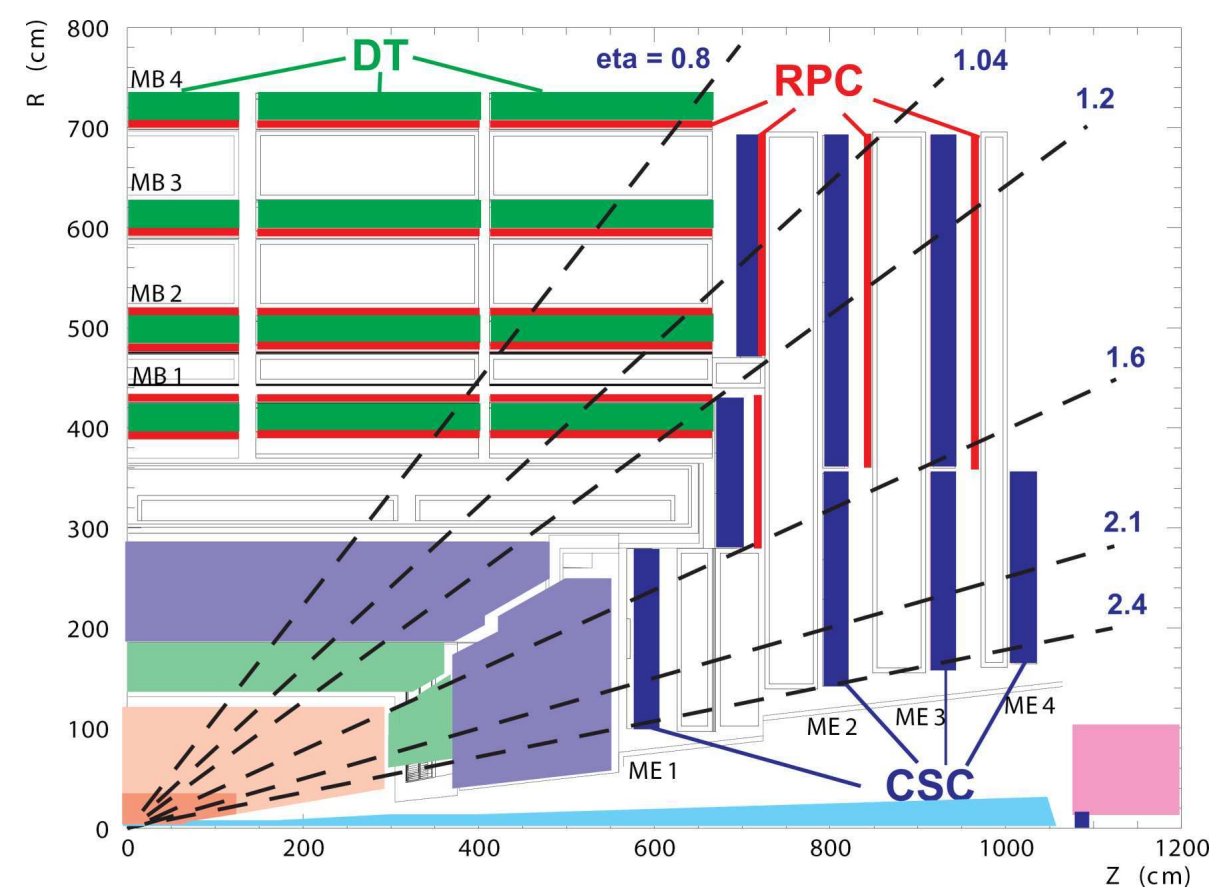

Figure 1.10: Layout of one quarter of the CMS muon system showing deployment of Drift Tubes (DTs), Cathode Strip Chambers (CSCs) and Resistive Plate Chambers (RPCs) integrated into the magnet return yoke. 


\section{The drift tube chambers}

The DT chambers were chosen for use in the barrel region where the muon rates and residual magnetic field are low. 250 DT chambers are organized in 4 concentric cylinders consisting of five wheels along the $z$-axis and cover a pseudorapidity region of $|\eta|<1.3$. Each wheel is divided into the $12 \phi$-sectors $\left(30^{\circ}\right)$ with 12 chambers in each of the 3 inner layers and 14 chambers in the outermost layer. Each chamber is made up of 12 layers of drift tubes organized in three independent units, so called super-layers (SL). Two SLs measure $\phi$-coordinate and the third measures the $z$-coordinate. The drift tube cell is a basic detector element having a rectangular shape and drift length of $2.1 \mathrm{~cm}$ corresponding to $\sim 400 \mathrm{~ns}$ drift time. The chosen gas mixture is a $85 \% / 15 \%$ of $\mathrm{Ar} / \mathrm{CO}_{2}$, which provides good quenching properties and a saturated drift velocity. A single cell has $99 \%$ efficiency with a single point resolution of $\sim 200 \mu \mathrm{m}$. Thus, a typical DT chamber resolution is $\sim 100 \mu \mathrm{m}[20]$.

\section{The cathode strip chambers}

The CSCs are used as a suitable muon detector for the endcap regions $(0.9<|\eta|<2.4)$, where the muon rates as well as the residual magnetic field are high. The CSC are multiwire proportional chambers with good spatial and time resolution. They have trapezoidal shape and are arranged in series of concentric rings centered on the beam line. Each ring consists of 18 or 36 chambers composed of 6 layers, each one consists of an array of anode wires between two cathode planes. One of the two cathode planes is segmented into strips orthogonal to the wires to provide $\phi$ measurement. The region among the cathodes is filled with a $40 \% / 50 \% / 10 \%$ mixture of $\mathrm{Ar} / \mathrm{CO}_{2} / \mathrm{CF}_{4}$ [20]. The spatial resolution provided by each chamber from the strips is typically $\sim 200 \mu \mathrm{m}(\sim 100 \mu \mathrm{m}$ for the innermost chamber $)$ and angular resolution in $\phi$ is of order $10 \mathrm{mrad}$ [21].

\section{The resistive plate chambers}

The RPCs are installed in both the barrel and the endcaps $(|\eta|<2.1)$. They have modest spatial resolution, but provide fast response with excellent time resolution and therefore identify unambiguous bunch crossing. There are 6 layers of rectangular chambers in the barrel and 4 layers of trapezoidal chambers in the endcaps. They are composed of four bakelite planes forming two coupled gaps filled with a $95.4 \% / 4.5 \%$ mixture of $\mathrm{C}_{2} \mathrm{H}_{2} \mathrm{~F}_{4} / \mathrm{i}-\mathrm{C}_{4} \mathrm{H}_{10}$ (freon/isobutane). The time resolution measured in the test beam is better than $1.6 \mathrm{~ns}$ [20].

\subsection{Conclusion}

In the first part of this chapter the Large Hadron Collider and its physics potential has been introduced. The LHC complex is now in the final stage and the first collision is foreseen to occur in fall 2008. In the second part, the individual subdetectors of the CMS experiment have been briefly described in order to show their design solutions and basic performances. The CMS detector has been installed at the Point 5 and its commissioning is under way. In the following chapter the CMS electromagnetic calorimeter will be presented. 



\section{Bibliography}

[1] O. S. Brüning et al., LHC Design Report. Geneva: CERN, 2004.

[2] L. Evans, "The large hadron collider (LHC)," New J. Phys., vol. 9, p. 335. mult. p, 2007.

[3] F. Gianotti, "Physics at the LHC," Phys. Rep., vol. 403-404, pp. 379-400, 2004.

[4] S. Abdullin, "Summary of the CMS potential for the higgs boson discovery," CERN, Geneva, Tech. Rep. CMS-NOTE-2003-033, Dec 2003.

[5] F. Gianotti, "Collider physics : LHC," CERN, Geneva, Tech. Rep. ATL-CONF-2000-001, Apr 2000.

[6] D. Costanzo, "Higgs physics at the Large Hadron Collider," CERN, Geneva, Tech. Rep. ATL-CONF-2001-002. hep-ex/0105033, May 2001, these are the proceedings of the talk I gave in Moriond. Fabiola already proofread it.

[7] CMS Collaboration, The Compact Muon Solenoid - Technical Proposal, ser. Technical Design Report CMS. Geneva: CERN, 1994, no. CERN/LHCC 94-38.

[8] C. collaboration, "The CMS experiment at the CERN LHC," Submitted to the Journal of Instrumentation (JINST), Jan 2008.

[9] ATLAS detector and physics performanceTechnical Design Report, 1, ser. Technical Design Report ATLAS. Geneva: CERN, 1999, electronic version not available.

[10] ATLAS detector and physics performanceTechnical Design Report, 2, ser. Technical Design Report ATLAS. Geneva: CERN, 1999, electronic version not available.

[11] LHCb : Technical Proposal, ser. Tech. Proposal. Geneva: CERN, 1998.

[12] ALICE Technical proposal for a Large Ion collider Experiment at the CERN LHC, ser. LHC Tech. Proposal. Geneva: CERN, 1995.

[13] V. Karimäki, The CMS tracker system project Technical Design Report, ser. Technical Design Report CMS. Geneva: CERN, 1997.

[14] M. Weber, "The CMS tracker," CERN, Geneva, Tech. Rep. CMS-CR-2004-046. CERNCMS-CR-2004-046, Oct 2004.

[15] N. De Filippis, "The performance of the CMS tracking," CERN, Geneva, Tech. Rep. CMSCR-2004-024, Jul 2004.

[16] The CMS hadron calorimeter project Technical Design Report, ser. Technical Design Report CMS. Geneva: CERN, 1997. 
[17] G. Baiatian et al., "Design, performance, and calibration of CMS hadron-barrel calorimeter wedges," CERN, Geneva, Tech. Rep. CMS-NOTE-2006-138. CERN-CMS-NOTE-2006-138, May 2007.

[18] The CMS magnet project Technical Design Report, ser. Technical Design Report CMS. Geneva: CERN, 1997.

[19] The CMS muon project: Technical Design Report, ser. Technical Design Report CMS. Geneva: CERN, 1997.

[20] P. Giacomelli, "The CMS muon detector," Nucl. Instrum. Methods Phys. Res., A, vol. 478, no. $1-2$, pp. $147-52,2002$.

[21] M. Della Negra et al., CMS physicsTechnical Design Report, ser. Technical Design Report CMS. Geneva: CERN, 2006. 


\section{Chapter 2}

\section{Electromagnetic calorimeter}

\subsection{Introduction}

The CMS Electromagnetic Calorimeter (ECAL) [1] consists of 75,848 lead tungstate $\left(\mathrm{PbWO}_{4}\right)$ radiation hard crystals. These crystals are distinguished with their high density $\left(8.28 \mathrm{~g} \mathrm{~cm}^{-3}\right)$, short radiation length $\left(\mathrm{X}_{0}=0.89 \mathrm{~cm}\right)$, and small Molière radius $\left(\mathrm{R}_{M}=2.19 \mathrm{~cm}\right)$ which allows to construct a compact highly granular calorimeter. The scintillation decay time is short (practically all the light is collected in $100 \mathrm{~ns}$ ) and well adapted to the $25 \mathrm{~ns}$ bunch crossing time. On the other hand, the relatively low light yield requires the use of photodetectors with intrinsic gain amplification able to work in a magnetic field of $4 \mathrm{~T}$. Hence Avalanche Photodiodes (APDs) are used in the central barrel region and, due to favorable orientation of the solenoid magnetic fild, Vacuum Phototriodes (VPTs) in the forward endcap regions. In addition, the sensitivity of both the crystal and photodetector response to the temperature fluctuations requires a precise control of the temperature stability. Finally, the small, but not negligible variation of the crystal transparency during irradiation needs a precise light monitoring system.

The first part of this chapter summarizes basic contributions to the energy resolution as a main characteristic of ECAL. In the second part, a short overview of the ECAL is given with respect to its mechanical design, basic properties of the $\mathrm{PbWO}_{4}$ crystals as its active medium, and chosen fotodetectors.

\section{$2.2 \quad$ Energy resolution}

The energy resolution of an homogenous calorimeter is usually parametrized as [1]:

$$
\frac{\sigma_{E}}{E}=\frac{S}{\sqrt{E}} \oplus \frac{N}{E} \oplus C,
$$

where the right-hand side is assumed to be the square root of the quadratic sum of the three terms: stochastic, noise and constant terms with coefficients $S, N, C$, respectively. The energy $\mathrm{E}$ is usually in $\mathrm{GeV}$ units. The relative importance of the various terms depends on the energy of the incident particle as can be seen in Fig 2.1 [1]. The different contributions to each term in Eq. 2.1 are discussed in detail below.

\subsubsection{Stochastic term}

The stochastic term is associated to statistical fluctuation in the number of primary processes that generate the signal and any other process that limits this number, i.e. due to the physical 


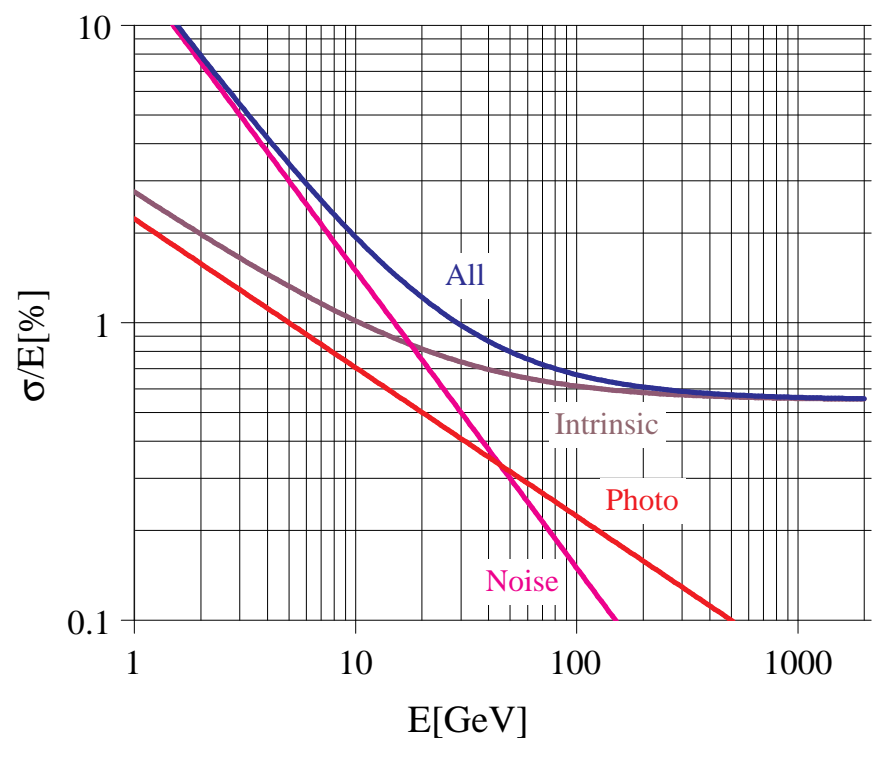

Figure 2.1: Total and different contributions to the energy resolution of the calorimeter. The curve labeled "intristic" includes both the shower containment and the constant term. The curve named "photo" represents stochastic contributions.

development of the electromagnetic shower in the calorimeter and successive scintillation light recollection. Therefore, the contributions to the stochastic term accounts mainly for the fluctuations on the lateral shower containment, fluctuation on the energy deposited in the material prior the calorimeter, and the photo-statistics contribution.

The lateral shower containment is due to the limited number of crystals involved in the energy reconstruction cluster. The cluster size is determined as a compromise between the gain in energy resolution from a large containment and the electronic noise of the read-out chain. For the ECAL the fluctuations of the lateral containment give a contribution of about $1.5 \% / \sqrt{E}$ if a $5 \times 5$ crystal array is considered.

The photo-statistics contribution includes effects related to the number of primary photoelectrons $N_{p e}$ released in the photodetector per unit of energy and the contribution from the multiplication process inside the photodetector, which are described by the excess noise factor $F$. Its values are about 2 and 3 for the APD and VPT, respectively. The $N_{p e}$ is given as a product of the scintillating light yield, light collection efficiency and photodetector quantum efficiency. Thus the photo-statistics contribution $S_{p e}$ can be written as in Eq. 2.2 and contributes about $2.3 \% / \sqrt{E}$ to the stochastic term of the ECAL.

$$
S_{p e}=\sqrt{\frac{F}{N_{p e}}} .
$$

In the case of the endcap, where the preshower detector is installed in front of the calorimeter, the contribution from the fluctuations on the energy deposited in the preshower material becomes dominant and gives a sampling term of about $5 \% / \sqrt{E}$. 


\subsubsection{Noise term}

The noise term accounts for the electronic noise of the read-out chain that depends on the detector technique and on the features of the read-out circuit, the radiation-induced dark current of the photodetectors, which rises with the life time of the experiment, and the physical noise due to the fluctuation in energy carried by particles coming from pile-up events. The contributions vary with pseudorapidty and with the luminosity of the machine. For a $5 \times 5$ crystal array in the barrel $(\eta=0)$, the expected contributions are $150 \mathrm{MeV}$ for the electronics noise, $30 \mathrm{MeV}$ and $110 \mathrm{MeV}$ for the leakage current and $30 \mathrm{MeV}$ and $95 \mathrm{MeV}$ for the pile-up at low and high luminosity, respectively. In the endcap $(\eta=2)$ the contribution to the noise term due to the electronics noise are $750 \mathrm{MeV}, 175 \mathrm{MeV}$ and $525 \mathrm{MeV}$ for the pile-up at low and high luminosity, respectively [1].

\subsubsection{Constant term}

The constant term includes contributions which do not depend on the energy of the particle and is the dominant term at high energies. The most important contributions come from the longitudinal non-uniformity of the light collection, the crystal-to-crystal intercalibration errors, the longitudinal shower containment and the instabilities and non-uniformities in the detector parameters, such as temperatures, high voltage distributed to the photodetectors, response changes induced by radiation, etc.

The longitudinal non-uniformity of the light collection is caused by two competing effects. The first is a light attenuation along crystals due to intrinsic absorption inside the material, diffusion on scatter centers and losses by multiple reflection on crystal faces. The second is due to a focusing effect caused by the tapered shape of the crystal and its high refractive index. This effect is dominant and can be effectively treated by depolishing one lateral face with a proper roughness. In order to keep the contribution to the constant term within $0.25 \%$, a slope of relative light yield variation in the shower maximum has to stay within a range from $-0.35 \% / X_{0}$ to $+0.35 \% / X_{0}[1,2]$.

Since an electromagnetic shower is not contained only inside the central crystal, but also among neighbouring crystals, and the lateral shower shape is nearly independent of energy, any effect due to imperfect relative crystal intercalibration goes directly into the constant term. In ECAL it will contribute $0.4 \%$.

The longitudinal shower containment appears when an energetic shower losses part of its energy beyond the rear face of the crystal. The length of the $\mathrm{PbWO}_{4}$ crystals used in ECAL are $23 \mathrm{~cm}\left(25.8 \mathrm{X}_{0}\right)$ and $22 \mathrm{~cm}\left(24.7 \mathrm{X}_{0}\right)$ in the barrel and endcap regions, respectively. Simulations and test beams studies have shown that this energy leakage together with uncorrected and imperfectly corrected geometrical effects are below $0.2 \%$.

Since the number of scintillation photons emitted by the crystals and the amplification of the APD are both temperature dependent, about $-4 \% /{ }^{\circ} \mathrm{C}$ at $18{ }^{\circ} \mathrm{C}$, the temperature stability within $\pm 0.05^{\circ} \mathrm{C}$ is required to keep the contribution to the constant term below $0.1 \%$. Moreover, as the APD gain strongly depends on the bias voltage $(1 / \mathrm{M} \mathrm{dM} / \mathrm{dV} \approx 3.2 \% / \mathrm{V}$ at gain $\mathrm{M}=50)$, the APDs require a bias voltage supply system with a stability bether than $30 \mathrm{mV}$ in order to give a contribution less than $1 \%$ to the constant term. The test beam experiments with final version of the cooling system and with the power supply regulation have fully met these requirements [3].

The targeted contributions of each term to the energy resolution in barrel and endcap for $5 \times 5$ crystal array at low and high luminosity are summarized in Tab. 2.1 [1]. 

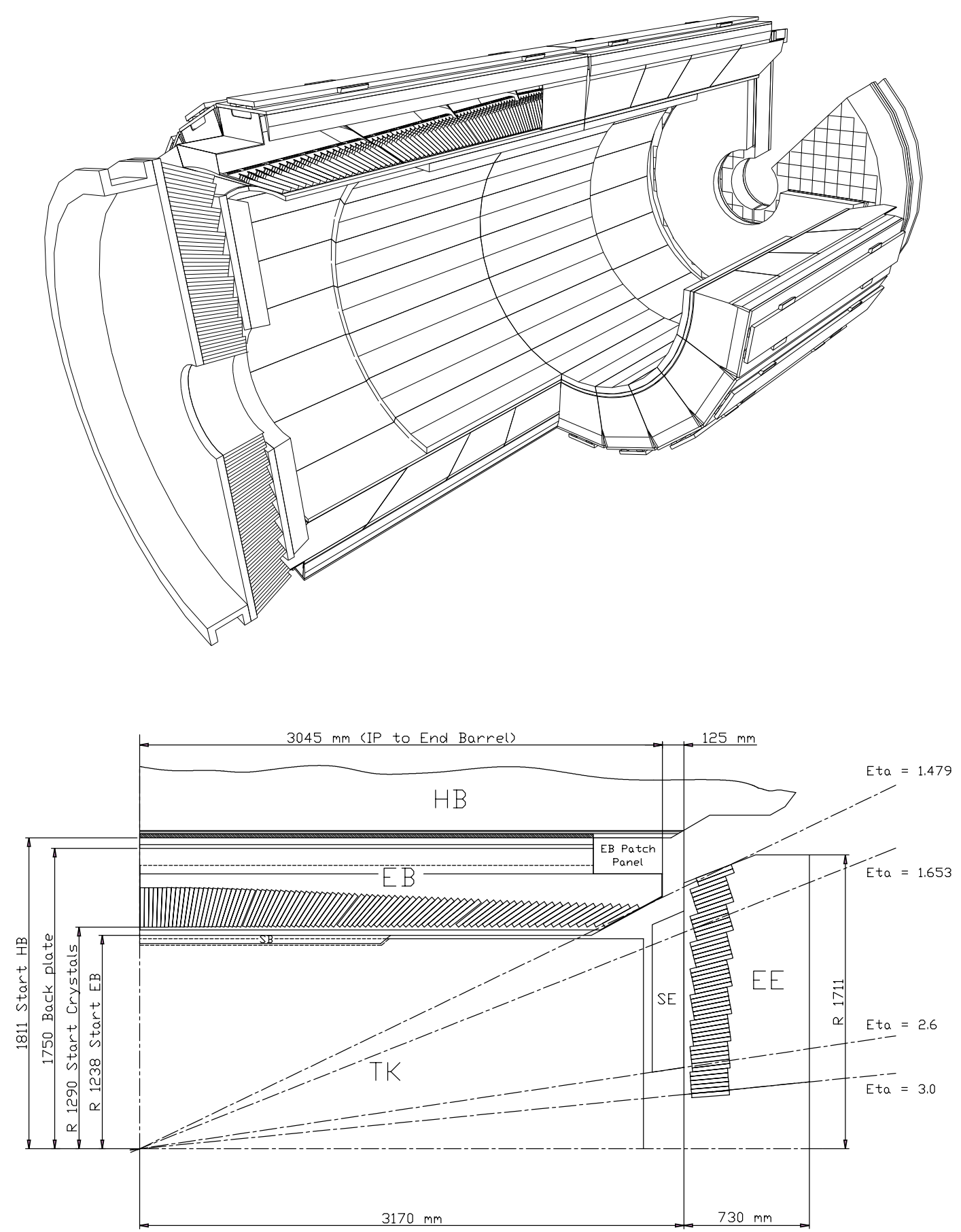

Figure 2.2: Overall view (top) and the one quadrant longitudinal section (bottom) of the CMS ECAL showing the arrangement of crystal modules, supermodules and endcaps. Here SE is the acronym for the pre-shower detector. 


\begin{tabular}{|l|c|c|}
\hline \hline Contribution & Barrel $(\eta=0)$ & Endcap $(\eta=2)$ \\
\hline Stochastic term & $2.7 \% / \sqrt{E}$ & $5.7 \% / \sqrt{E}$ \\
Noise term & $0.155 \mathrm{GeV}(0.210 \mathrm{GeV})$ & $0.770 \mathrm{GeV}(0.915 \mathrm{GeV})$ \\
Constant term & $0.55 \%$ & $0.55 \%$ \\
\hline \hline
\end{tabular}

Table 2.1: The targeted contributions to the energy resolution of the CMS ECAL in barrel and endcap for the $5 \times 5$ crystal array at low (high) luminosity.

\subsection{Calorimeter design}

An overall view of the ECAL is given in Fig. 2.2. It is subdivided in a barrel part (EB) and two endcaps (EE), with in addition a pre-shower detector (ES) in front of the latter.

\subsubsection{Barrel}

The barrel part of the calorimeter is constructed according to an off-pointing geometry of $3^{\circ}$ in each of $\eta$ and $\phi$ towards the interaction point and covers a pseudo-rapidity interval of $|\eta| \leq$ 1.479. It consists of 61,200 crystals of 17 different sizes, which are organized in two rings of 18 supermodules each. The crystals have a tempered shape, slightly varying along the polar angle with respect to the beam axis with a mean dimension of $2.2 \times 2.2 \times 23 \mathrm{~cm}^{3}$ corresponding to $\sim 25.8 \mathrm{X}_{0}$. The crystals are grouped into $2(\phi) \times 5(\eta)$ matrices, held in a glass fiber alveolar submodule that is the smallest unit in the barrel with all crystals of identical shape. The 40 or 50 submodules are then mounted into a module held by an aluminum grid. Four modules, one module containing 50 submodules (500 crystals) and the other three with 40 submodules (400 crystals) each are assembled together to form a supermodule, which thus contains 1,700 crystals. All the barrel supermodules mounted in final position inside the barrel HCAL in the CMS experimental hall in 2006 are shown in Fig. 2.3.

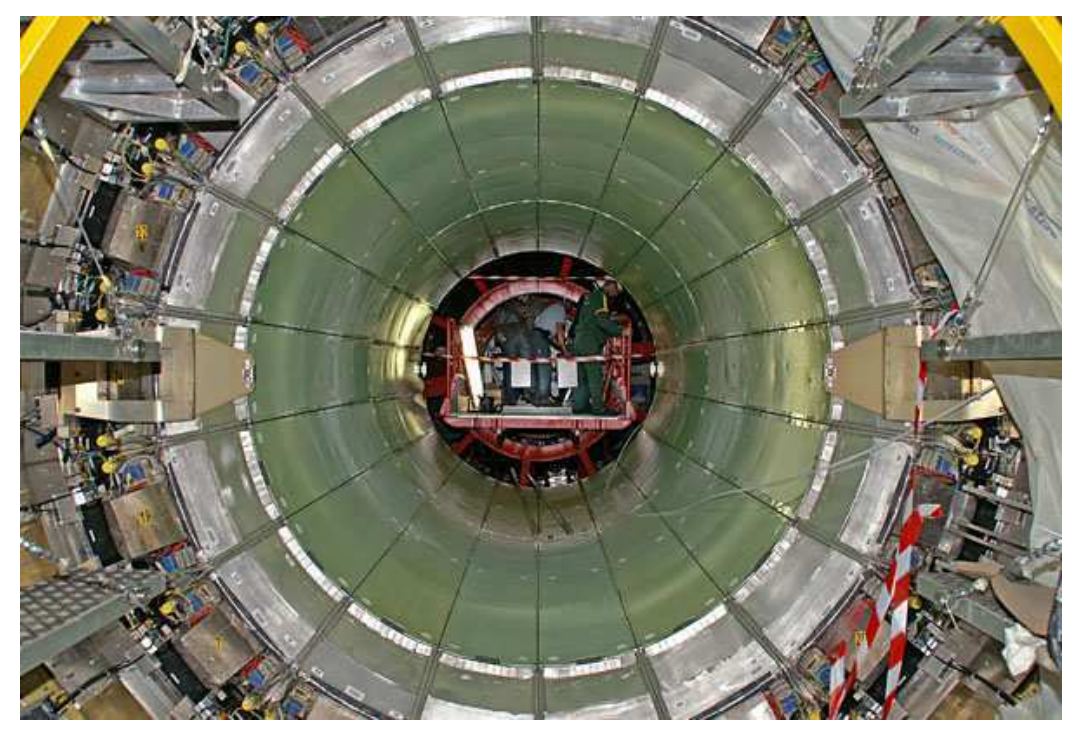

Figure 2.3: All barrel supermodules mounted in their final possition inside the CMS hadron calorimeter. 


\subsubsection{Endcaps}

The endcaps of the calorimeter consist of a total of 14,648 identical crystals organized in four "Dees" containing 3,662 crystals each. $5 \times 5$ crystals that are held in a carbon fiber alveolar form a super-crystal (138 standard super-crystals and 18 special partial super-crystals on the inner and outer circumference). The crystals and super-crystals are arranged in a rectangular $x, y$ grid, with the crystals pointing at a focus $1,300 \mathrm{~mm}$ beyond the interaction point, so that the off-pointing angle varies with $\eta$. The crystals have a slightly larger cross-section $\left(3 \times 3 \times 22 \mathrm{~cm}^{3}\right)$ than the barrel crystals and a radiation length of $\sim 24.7 X_{0}$. The endcap covers a pseudo-rapidity interval of $1.479<|\eta|<3$.

\subsubsection{Pre-Shower}

The main aim of the pre-shower detector is to identify neutral pions $\pi^{0}$ in the endcaps within a fiducial region $1.653<|\eta|<2.6$. It also helps the identification of electrons against minimum ionizing particles, improves the position determination of electrons and photons with its superior granularity and reduces the longitudinal leakage from energetic showers.

The pre-shower is a sampling calorimeter with 2 layers: lead radiators initiate electromagnetic showers from incoming photons/electrons whilst $1.9 \mathrm{~mm}$ pitch silicon strip sensors placed after each radiator measure the deposited energy and the transverse shower profiles. The material thickness of the first and the second radiator are $\sim 2 \mathrm{X}_{0}$ and $\sim 1 \mathrm{X}_{0}$, which gives a total absorber thickness of $\sim 3 \mathrm{X}_{0}$. Fig. 2.42.4 shows the detailed longitudinal structure of the $\mathrm{SE}$ and a detailed description of the pre-shower detector can be found in Ref. $[4,5]$.

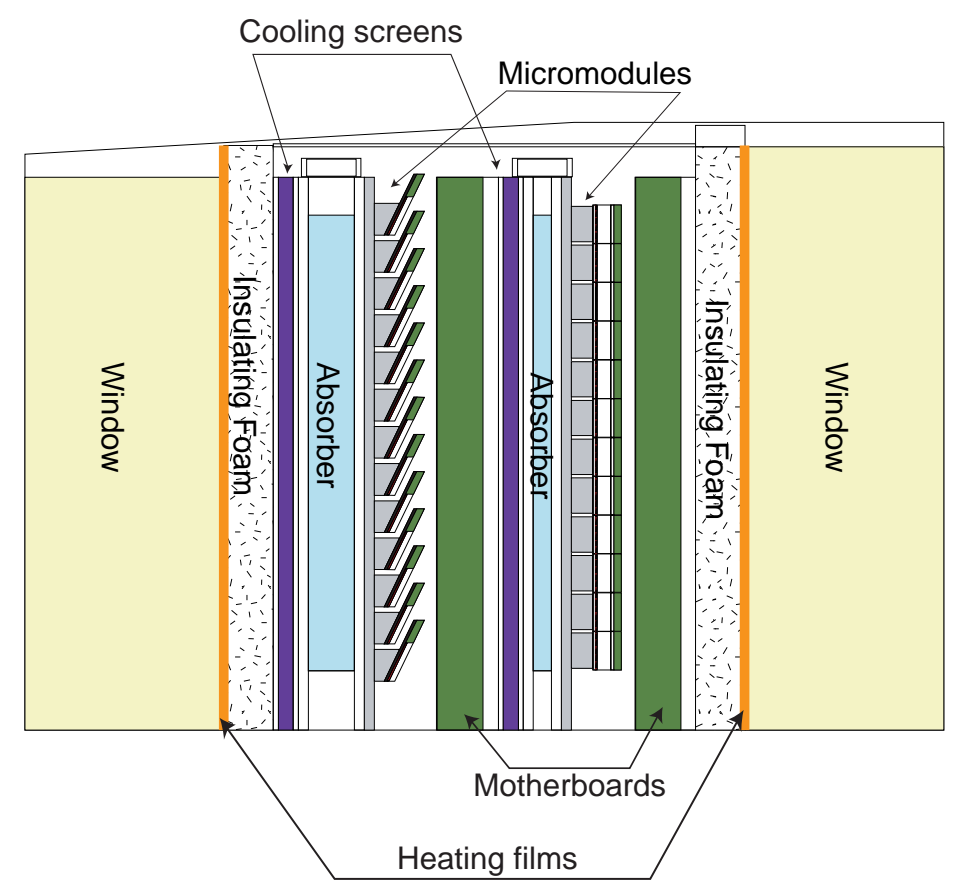

Figure 2.4: The schematic longitudinal cross-section of the SE, with the two $1 \mathrm{~cm}$ thicknesses of foam and the heating films [6]. 
The performance of the preshower detector was evaluated in a test beam on a supercrystal $\left(5 \times 5\right.$ crystal array) prototype with a total absorber thickness of $\sim 3.24 \mathrm{X}_{0}$ [7]. The measured spatial precision as a function of the electron energy is approximated as:

$$
\sigma(\mu m)=\frac{1649}{\sqrt{E(G e V)}} \oplus 193 .
$$

If the preshower is taken into account, the total energy $E_{t o t}$ in $\mathrm{GeV}$ deposited in the endcap is defined as:

$$
E_{\text {Tot }}=E_{X \text { tals }}+E_{S E},
$$

where $E_{X t a l s}$ is the energy deposited in a crystal array $(3 \times 3$ or $5 \times 5)$ and $E_{S E}$ is the energy deposited in preshower and in the case of two planes is given as:

$$
E_{S E}=\gamma\left(E_{1}+\alpha E_{2}\right),
$$

$E_{1}, E_{2}$ (expressed in MIPs) are the charge deposited in each plane of the preshower summed on 5 strips centered on the strip with the highest energy. The $\gamma$ is the coefficient of conversion between $\mathrm{GeV}$ and MIPs and coefficient $\alpha$ is a relative weight of each plane and is adjusted in order to get the best energy resolution. The pre-shower contribution as a function of energy, which should be added in quadrature to the energy resolution of the endcap alone, measured during the test beam is parametrized as:

$$
\sigma_{E}=\frac{3.96 \%}{\sqrt{E(G e V)}} \oplus 0.49 \%
$$

\subsection{Crystals}

The choice of the $\mathrm{PbWO}_{4}$ crystals was mainly driven by their favorable physical properties mentioned above and which are summarized in Tab. 2.2. Since the radiation environment for the CMS ECAL is very hostile (dose rates from 0.15 to $0.3 \mathrm{~Gy} \mathrm{~h}^{-1}$ in the barrel and from 0.3 to $15 \mathrm{~Gy} \mathrm{~h}^{-1}$ in the endcap) the main focus of the $\mathrm{R} \& \mathrm{D}$ was to improve radiation tolerance of the crystals. Improvements in radiation hardness were reached by stochiometric fine-tuning, optimization of crystal growth conditions and doping with yttrium and niobium [8]. It was found that radiation does not affect the scintillation mechanism in the $\mathrm{PbWO}_{4}[9]$ and the loss of the light output is only due to the radiation-induced absorption, i.e. to the formation of color centers, which reduce the crystal transparency. Because only the transparency is affected, it is possible to monitor the loss in transmission due to irradiation using a light injection system in the calorimeter and to apply corrections for it [10]. A brief description of the laser monitoring system is given later in Chap. 2.6.

Moreover, the R\&D has led to a significant improvement in optical properties of the crystals. The optical transmission has been considerably improved in the whole scintillation region (360 - $570 \mathrm{~nm}$ ) and by more than a factor of 2 at the emission peak as shown in Fig. 2.5 (top) for two typical crystals produced in years 1995 and 1997 [1]. The transparency improvement is associated with an increase in the amount of collection light and, as a consequence, the light yield has increased by more than $50 \%$ on average and reached $12 \mathrm{pe} / \mathrm{MeV}$ for the first preproduction batch in 1998 [13].

The main drawbacks of the $\mathrm{PbWO}_{4}$ crystals are their relatively low light yield that is three orders of magnitude less than $\mathrm{NaI}$ and a high temperature dependence of $\sim-2 \% /{ }^{\circ} \mathrm{C}$ at room temperature. Both are due to a strong thermal quenching of the scintillation mechanism, which 


\begin{tabular}{|l|c|}
\hline \hline Parameter & Value \\
\hline Density $\left[\mathrm{g} \cdot \mathrm{cm}^{3}\right.$ ] & 8.28 \\
Radiation length $[\mathrm{cm}]$ & 0.89 \\
Molière radius $[\mathrm{cm}]$ & 2.19 \\
Refractive index $(\lambda=632 \mathrm{~nm})$ & 2.16 \\
Scintillating light emission $[\mathrm{nm}]$ & 420 \\
Light yield [pe/MeV] & 12 \\
Light decay time $[\mathrm{ns}]$ & $\sim 10$ \\
$(80 \%$ of the light is colected in $25 \mathrm{~ns})$ & \\
\hline \hline
\end{tabular}

Table 2.2: Basic properties of the $\mathrm{PbWO}_{4}$ scintillating crystals of the CMSECAL $[1,11]$.

leads to a fast but relatively low light yield and its rather strong temperature dependence. The light yield and its temperature coefficient are shown in Fig. 2.5 (bottom) as a function of temperature in a range from -20 to $50{ }^{\circ} \mathrm{C}$ [12]. The low yield can be compensated by use of photodetectors with intrinsic gain amplification (described in next Chap. 2.5), whilst the temperature dependence requires a cooling system keeping the crystal temperature stable at $18^{\circ} \mathrm{C}$ within $\pm 0.05^{\circ} \mathrm{C}$ to preserve energy resolution.

The Bogorodisk Techno-Chemical Plant $(\mathrm{BTCP})^{1}$ and the Shanghai Institute of Ceramics $(\mathrm{SIC})^{2}$ have manufactured all the $\mathrm{PbWO}_{4}$ crystals for the CMS ECAL. The reproducibility of the crystal characteristics with acceptable values in terms of geometry, optical parameters as well as resistance to radiation is the main goal in the production. To check these parameters and fulfill selection criteria, each single crystal is characterized with the Automatic Crystal COntrol System (ACCOS) $[14,15]$ which was built in two various versions at CERN and INFN/ENEA Rome regional centers. On each crystal the ACCOS measures its dimensions, longitudinal and transversal transmission as a function of wavelength, light yield and uniformity of light collection and compares measured values with specifications set to guarantee the final quality of the calorimeter. All the data collected are registered in the CMS database REDACLE (Relational Ecal Database for Construction Level) [16] and in ECAL global database CRISTAL (Cooperating Repositories and Information System for Tracking Assembly Lifecycles) [17], where they are accessible at any time to control the production status.

A status of the first 15,000 production crystals which were characterized by ACCOS at the CERN Regional Centre can be found in Ref. [14]. From this study the crystal rejection rate is expected at a level of $\sim 1 \%$, that corresponds to following selection criteria:

- The longitudinal light transmission at $420 \mathrm{~nm}$ (the scintillation emission peak value) must be at least $55 \%$ in order not to affect the light collection uniformity in the crystal.

- The light yield front non-uniformity in the region of shower maximum must be less then $0.35 \% / \mathrm{X}_{0}$ in order to achieve the maximal targeted contribution of $0.3 \%$ to the resolution constant term.

- The light yield measured at $8 \mathrm{X}_{0}$ must be at least 8 photo-electron/MeV.

- The light yield loss of the sample-irradiated crystals $\left(0.15\right.$ Gy.h $\left.{ }^{-1}\right)$ must not exceed $6 \%$.

\footnotetext{
${ }^{1}$ Tula, Russia

${ }^{2}$ Shanghai, China
} 

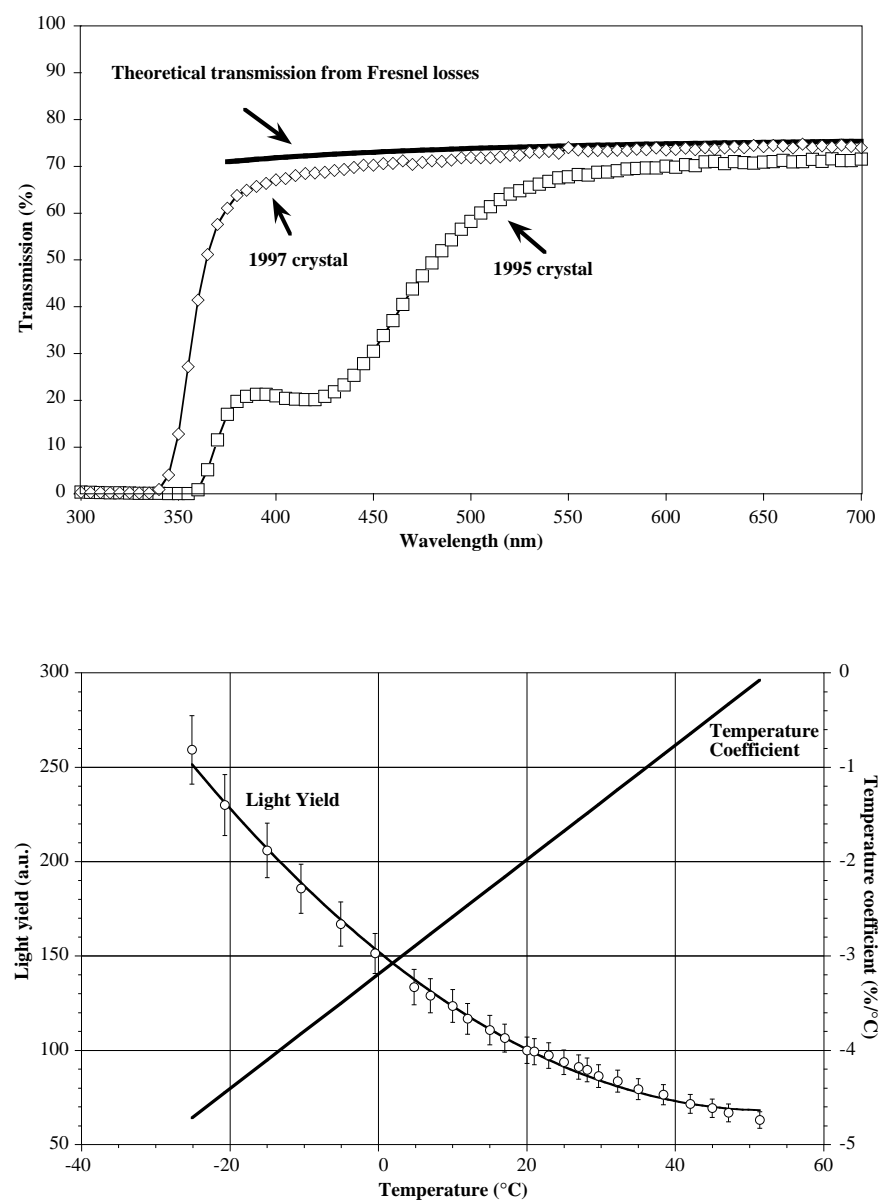

Figure 2.5: Top: Progress in the longitudinal transmission spectrum of the $\mathrm{PbWO}_{4}$ crystal between the years 1995 and 1997 in comparison with maximum achievable transmission [1]. Bottom: Temperature dependency of the light yield and temperature coefficient of the $\mathrm{PbWO}_{4}$ crystal [12].

\subsection{Photodetectors}

\subsubsection{Avalanche photodiodes}

As mentioned earlier, because of the relatively low light yield of $\mathrm{PbWO}_{4}$, photodetectors with intrinsic amplification are needed. In addition, they have to be fast $(\leq 10 \mathrm{~ns})$, radiation hard $\left(2 \times 10^{13}\right.$ neutrons $/ \mathrm{cm}^{2}$ and $\left.2.5 \mathrm{kGy}\right)$ and able to operate in a strong magnetic field of $4 \mathrm{~T}$. For the barrel, these conditions are met by Avalanche Photodiodes (APDs), type S8148 reverse structure, as the fruit of the 10-year R\&D effort between the collaboration and Hamamatsu Photonics ${ }^{3}$.

The principle of operation of the APD is the following: Scintillation photons enter the

\footnotetext{
${ }^{3}$ Hamamatsu City, Japan
} 
device and convert in a $\mathrm{p}++-$ layer; these conversion electrons are then accelerated by a stronggradient electric field in a p-layer. This field peaks at a p-n junction so as to induce avalanche multiplication of the electrons, which then drift under constant electric field in an n-region and are collected in an $\mathrm{n}++-$-region as the photoelectric current.

The important characteristic is the nuclear counter effect or the electrical signal that is generated by ionizing particles traversing the APD, and can be quantified by means of effective thickness $\mathrm{d}_{\text {eff }}$ of the APD as a thickness of a standard silicon PN diode required to give the same signal. Since only the electrons generated in the p-region in front of the p-n junction will produce significant electrical signals, the nuclear counter effect strongly depends on the thickness of the p+ -layer.

The APDs affect all three terms of the energy resolution: the stochastic term through internal avalanche multiplication characterized by the excess noise factor $\mathrm{F}$, the constant term by the gain variation with bias voltage and temperature, and the noise term due to APD capacitance and dark current [18].

The APD capacitance contributes to the serie noise as:

$$
\frac{\sigma(M e V)}{E} \propto \frac{C_{D}+C_{P A} \sqrt{4 k T\left[R_{s}\left(\frac{C_{D}}{M}\right)^{2}\right]+\frac{0.7}{g_{m}}}}{q \sqrt{8} N_{\text {p.e. }} M E}
$$

where $C_{D}$ and $C_{P A}$ are the capacitances of the APD and amplifier, $R_{s}$ is the series resistance, $M$ is the gain, $g_{m}$ is the transconductance, $\tau$ is the shaping time, is the $k$ is the Boltzmann constant, and $T$ is the temperature.

The contribution to the noise term due to the dark current (parallel noise), which becomes more important with increasing radiation level, is:

$$
\frac{\sigma(M e V)}{E} \propto \frac{\sqrt{2 q\left(I_{s}+F M^{2} I_{b}\right) \tau}}{q \sqrt{8} N_{p . e . M E}},
$$

where $I_{s}$ and $I_{b}$ are the surface and the bulk contribution to the dark current. It is clear that to minimize the noise contribution, the primary goals of R\&D were to decrease APD capacitance and to reduce the dark current and the excess noise factor. The final physical and electrical parameters of the APDs are summarized in Tab. 2.3 and a schematic cross-view of the APD is shown in Fig. 2.6 right [19].

Radiation induced changes on the APDs occur through two mechanisms: the surface damage which causes defects in the front layer, and the bulk damage, due to displacements of atoms from their lattice site. The first increases the surface dark current and changes the quantum efficiency, while the second increases the bulk dark current that may change the gain. The APD radiation hardness has been proven for doses two times higher than those expected in the ECAL barrel. For most of the APDs, only the expected increase in the dark current has been seen, whilst any other changes in their electrical properties have not been observed [18]. It is expected that after 10 years of LHC running, the dark current will rise to a noise energy equivalent of $50 \mathrm{MeV}$ for a crystal.

Two APDs connected in parallel constitute a "capsule" (see Fig. 2.6 left) which is glued on the rear face of the crystal and thus provides an active area of $2 \times 25 \mathrm{~mm}^{2}=50 \mathrm{~mm}^{2}$. Because of the strong temperature variation of the APD gain $\left(1 / \mathrm{M} \cdot \mathrm{dM} / \mathrm{dT} \approx-2.4 \% /{ }^{\circ} \mathrm{C}\right.$ for gain $\left.\mathrm{M}=50\right)$, each $10^{\text {th }}$ capsule is equipped with a radiation-hard temperature sensor. Since the capsules, once glued to the crystal and mounted inside the calorimeter, become essentially inaccessible during 


\begin{tabular}{|l|c|}
\hline \hline Parameter & APD value \\
\hline Sensitive area & $5 \times 5 \mathrm{~mm}^{2}$ \\
Charge collection within $20 \mathrm{~ns}$ & $99 \pm 1 \%$ \\
Capacitance & $75 \mathrm{pF}$ \\
Serial resistance & $<5 \Omega$ \\
Quantum efficiency at $430 \mathrm{~nm}$ & $75 \pm 5 \%$ \\
Operating gain $\mathrm{M}$ & 50 \\
Operating voltage $\mathrm{V}_{r}$ & $340-410 \mathrm{~V}$ \\
Dark current $\mathrm{I}_{d}$ at $\mathrm{V}_{r}$ & $\leq 50 \mathrm{nA}$ \\
Breakdown - operating voltage $\left(\mathrm{V}_{b}-\mathrm{V}_{r}\right)$ & $45 \pm 5 \mathrm{~V}$ \\
Voltage sensitivity at $\mathrm{V}_{r}(1 / \mathrm{M} . \mathrm{dM} / \mathrm{dV})$ & $\leq 3.5 \% / \mathrm{V}$ \\
Temperature sensitivity at $\mathrm{V}_{r}(1 / \mathrm{M} . \mathrm{dM} / \mathrm{dT})$ & $-2.4 \pm 0.2 \% /{ }^{\circ} \mathrm{C}$ \\
Excess noise factor $\mathrm{F}$ at $\mathrm{V}_{r}$ and $430 \mathrm{~nm}$ & $2 \pm 0.3$ \\
Nuclear counter effect $\mathrm{d}_{e f f}$ & $<7 \mu \mathrm{m}$ \\
\hline \hline
\end{tabular}

Table 2.3: Main properties of the Avalanche Photodiode developed for the CMS ECAL $[19,20]$.
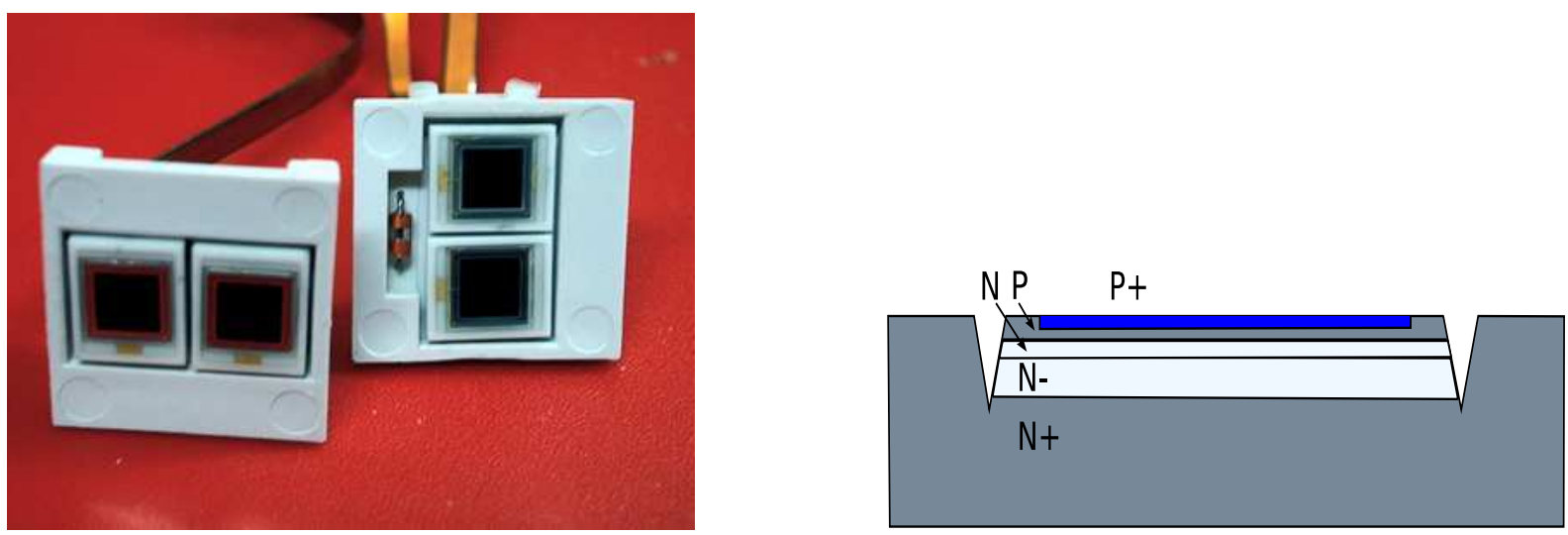

Figure 2.6: Left: A photography of a capsule assembled with two APDs. Right: Schematic view of principle of an APD. 
the whole life-time of the experiment, the crucial QA program is applied in order to guarantee a maximum APD failure rate of $1 / 1000$. To fulfill this criterion, all the APDs have to pass a predictive screaming program $[20,21]$ involving a $5 \mathrm{kGy}$ pre-irradiation at a dose rate of 2.5 $\mathrm{kGy} \mathrm{h}^{-1}$ with a ${ }^{60} \mathrm{Co}$ gamma source at PSI followed by combined annealing at a temperature of $80^{\circ} \mathrm{C}$ for 4 weeks and accelerated ageing under bias at $350 \mathrm{~V}$ at CERN. The APD are also sample-irradiated with a neutron source having a flux of $2 \times 10^{13}$ neutrons. $\mathrm{cm}^{-2}$. Only the APDs which passed pre-defined selection criteria for critical parameters are retained: the difference between breakdown voltage before and after the pre-radiation (maximum tolerance is $5 \mathrm{~V}$ ), dark current $\mathrm{I}_{d}, \mathrm{I}_{d} / \mathrm{M}$ vs. $\mathrm{M}$, where $\mathrm{M}$ is operating gain, and noise.
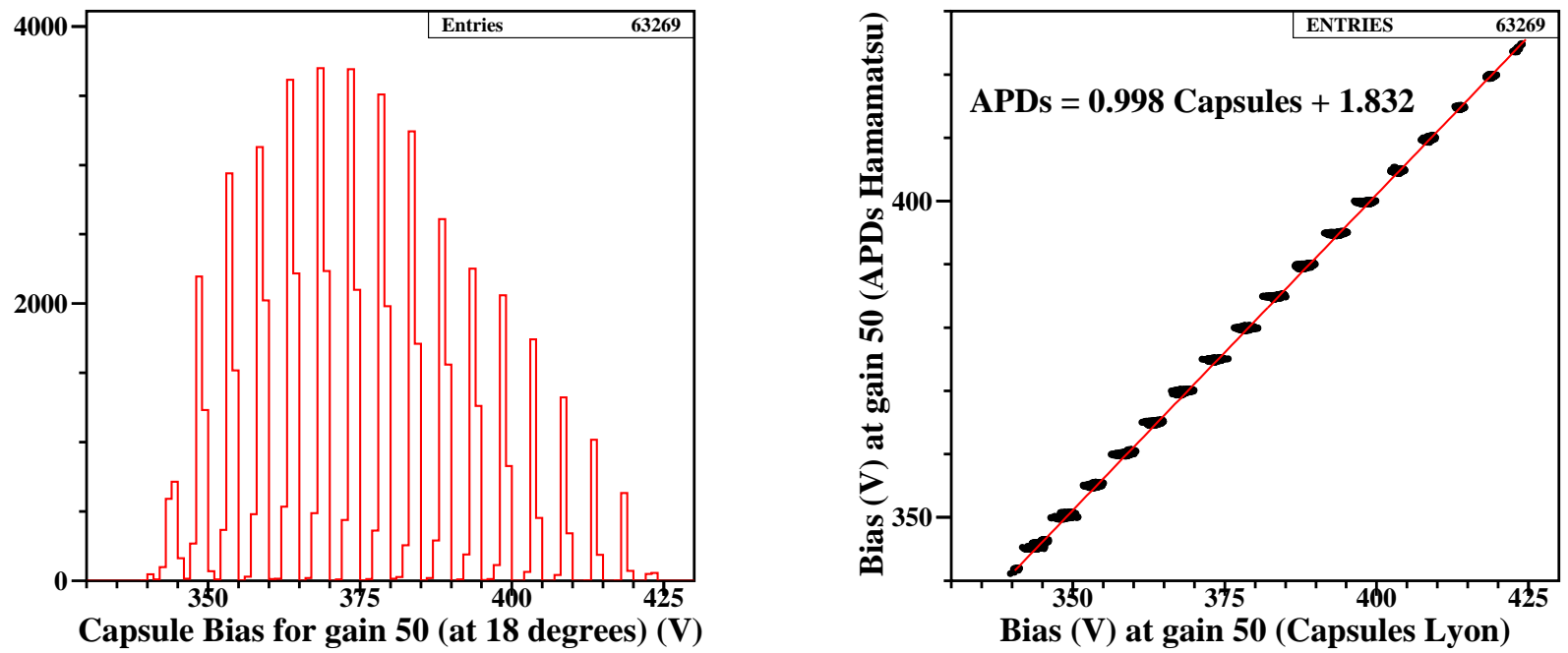

Figure 2.7: Left: Distribution of the APD bias voltage at gain 50. The structure in the distribution is caused by the APD pairing process in $5 \mathrm{~V}$ bins. Right: Correlation between the bias voltage at gain 50 for the ADPs and the capsules.

The retained APDs were sent to IPN Lyon, where, already grouped into pairs according to their values of bias voltage at gain 50, have been assembled into the capsules and calibrated. The grouping of APDs is required by the ECAL high-voltage power supply system. Each module of this system distributes the voltage to 100 APDs (50 capsules). The calibration was performed on a fully automatic calibration bench [22] developed at IPN Lyon, where the main following characteristics were measured: the gain versus bias voltage, the dark current $\mathrm{I}_{d}$ at gain 50 , a low and high frequency noise at different gains $(50,70,150)$. Moreover, a charge pulse was applied in order to check the rise and fall time as well as peak-to-peak signal value. As an example of measured variables for all APDs, a distribution of bias voltage at gain 50 is shown in Fig.2.7 left. Fig.2.7 right depicts a correlation between bias voltage measured on the APDs at the manufacturer and on the capsules at IPN Lyon and showing very good consistency in obtained results. The capsules rejection rate is $0.5 \%$ [23].

\subsubsection{Vacuum phototriodes}

Since the radiation dose and neutron fluences are one order of magnitude higher in the endcaps than in the barrel, silicon detectors are not suitable for use in the endcap region. On the other 
hand, the favorable orientation of the magnetic field in this region leads to the choise of Vacuum Phototriode (VPT) as the photodetector. The VPTs [24], type PMT188, which were developed in collaboration with the Research Institute Electron (RIE) ${ }^{4}$, are practically single-stage photomultiplier tubes with fine-mesh anodes equipped with radiation-hard ultraviolet windows. One VPT is glued to the back of each crystal. They have, as well as APDs, an internal gain (a mean gain of $\sim 10$ at $\mathrm{B}=0 \mathrm{~T}$ and $\sim 8$ at $\mathrm{B}=4 \mathrm{~T}$ ) and an optimized area to cope with the modest light yield of the crystal. The VPTs with an external diameter $25 \mathrm{~mm}$ have about the same order of total efficiency (quantum efficiency $\times$ sensitive photocatode area) for the detection of the crystal scintillation light as $50 \mathrm{~mm}^{2}$ APDs with several times higher quantum efficiency.
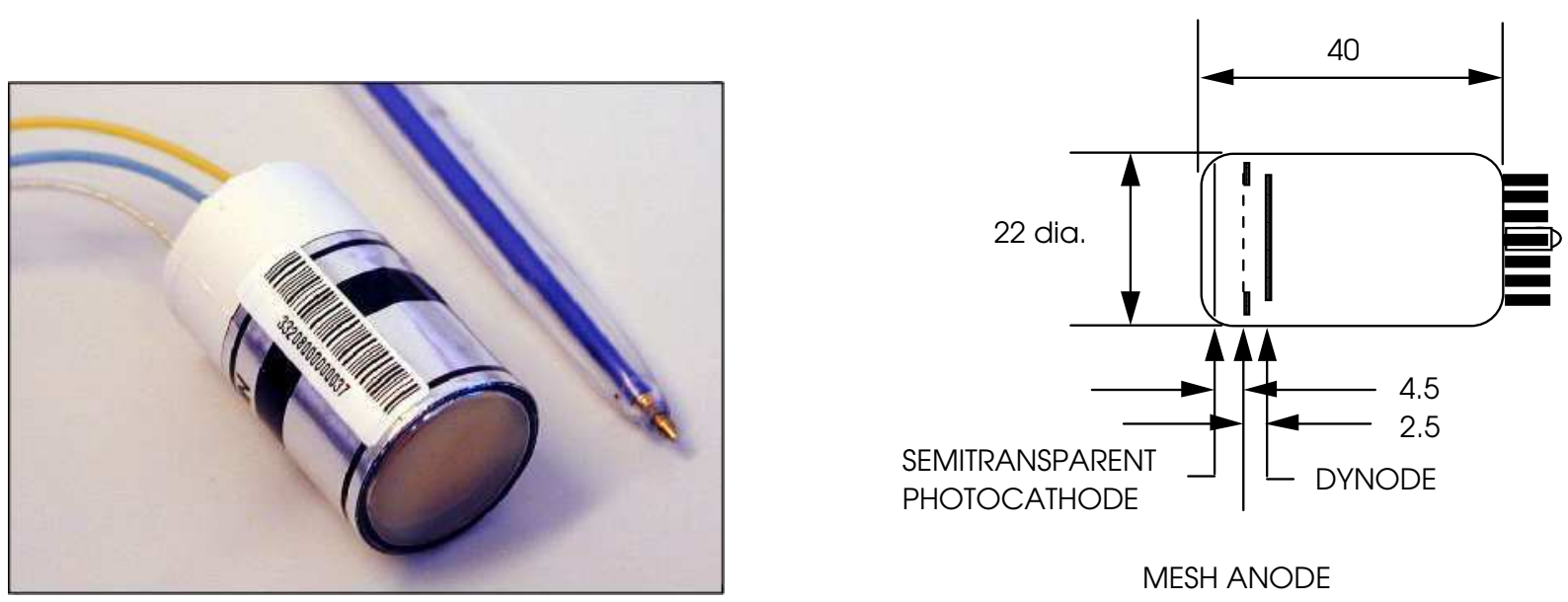

Figure 2.8: Left: A photograph of the CMS vacuum phototriodes (VPT). Right: Schematic view of the VPT.

Fig. 2.8 illustrates schematically the VPT construction (right) and shows a photograph of the VPT (left). Optical photons coming from crystals strike a planar semi-transparent cesiumantimony (SbKCs) photocathode deposited on the inner surface of the glass faceplate. The emitted photoelectrons are then accelerated towards the anode, which consists of a very fine copper mesh $(10 \mu$ pitch $)$ allowing VPTs to operate in a high magnetic field of $4 \mathrm{~T}$. A fraction (typically $\sim 50 \%$ ) of the electrons pass through the anode and strike the solid metal dynode coated with the same bialkali material as the photocathode, located just behind the anode. Secondary electrons ejected from the dynode are accelerated back towards the anode, where most are collected. The dynode under operating conditions $\left(\mathrm{V}_{a}=1000 \mathrm{~V}, \mathrm{~V}_{d}=800 \mathrm{~V}\right)$ has a secondary emission coefficient of approximately 20. Thus, taking into account the transparency of the anode mesh, the effective gain of the VPT is an order of 10. A more complete discussion of the operation is given elsewhere $[24,25]$.

The main operating parameters and properties of the VPT are listed in Tab. 2.4. The VPTs are, contrary to the APDs, very weakly dependent on the electrode bias voltage $(1 / \mathrm{M} . \mathrm{dM} / \mathrm{dV}$ $<0.1 \% / \mathrm{V})$, they are less sensitive to the temperature variations $\left(1 / \mathrm{M} . \mathrm{dM} / \mathrm{dT}<0.1 \% /{ }^{\circ} \mathrm{C}\right)$, and they are much more radiation hard. The radiation hardness of the VPT has been verified in gamma radiation fields up to $50 \mathrm{kGy}$ and neutron fluence of $7 \times 10^{15}$ neutron.cm ${ }^{-2}$ [25]. It

\footnotetext{
${ }^{4}$ St. Petersburg, Russia
} 


\begin{tabular}{|l|c|}
\hline \hline Parameter & VPT value \\
\hline Active area & $280 \mathrm{~mm}^{2}$ \\
Quantum efficiency at $430 \mathrm{~nm}$ & $22 \%$ \\
Operating gain $\mathrm{M}($ at $4 \mathrm{~T})$ & $8-10$ \\
Operating voltages $\mathrm{V}_{a}, \mathrm{~V}_{d}$ & $1000,800 \mathrm{~V}$ \\
Dark current $\mathrm{I}_{d}$ & $<1 \mathrm{nA}$ \\
Excess noise factor $\mathrm{F}$ at $420 \mathrm{~nm}$ & 3 \\
Voltage sensitivity $(1 / \mathrm{M} . \mathrm{dM} / \mathrm{dV})$ & $<0.1 \% / \mathrm{V}$ \\
Temperature sensitivity $(1 / \mathrm{M} . \mathrm{dM} / \mathrm{dT})$ & $<0.1 \% /{ }^{\circ} \mathrm{C}$ \\
Range of spectral response & $300-620 \mathrm{~nm}$ \\
\hline \hline
\end{tabular}

Table 2.4: Main properties of the Vacuum Phototriodes developed for the CMS ECAL $[5,24,25]$.

was shown that anode response variation is fully determined by the loss of the VPT faceplate transmittance during gamma irradiation and does not exceed $7 \%$ at $20 \mathrm{kGy}$ (maximum dose for $\eta=2.6$ ) for the chosen glass faceplate and the excess noise factor increases only by $10-12 \%$ at gamma dose of $50 \mathrm{kGy}(\eta=3.0)$.

Since the VPTs will operate in a high magnetic field, it was necesary to accurately evaluate their performance in a magnetic field up to $4 \mathrm{~T}$. It was found that the changes of the gain and excess noise factor $F$ at $4 \mathrm{~T}$ are around $10 \%$, see Fig. 2.9. The gain and $F$ were also measured for different tilt angles in different magnetic fields. The spread up to $10 \%$ in measured values was observed and explained by different orientations of the fine mesh plane of the VPTs relative to the axis of the device. Because it is not possible to achieve the same VPT orientation during the endcap assambly, this variation has to be considered as a supplementary factor in the energy resolution [25].

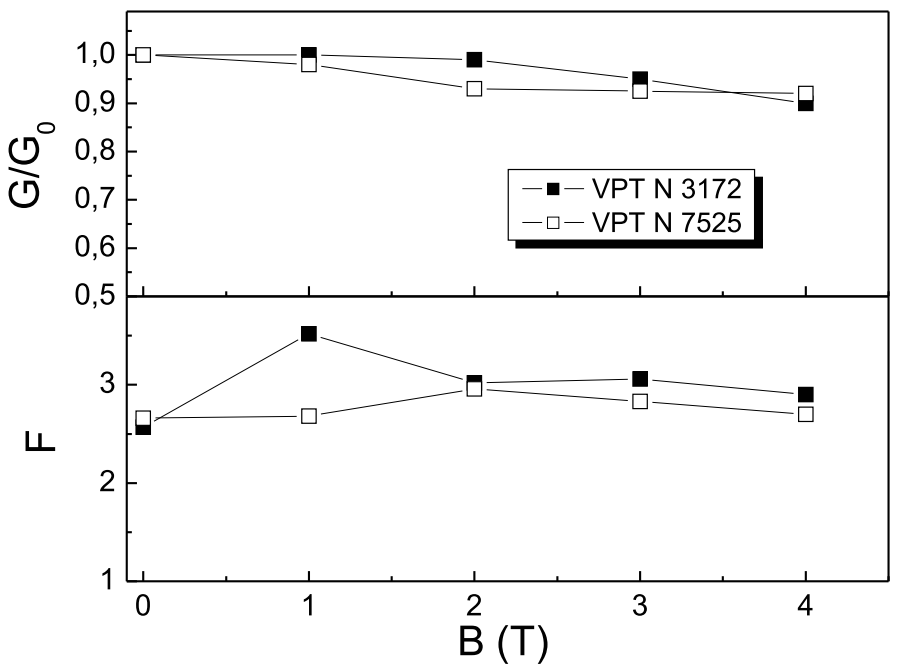

Figure 2.9: Relative gain $G / G_{0}$ and excess noise factor $F$ as a function of the magnetic field [25]. 
The decrease in VPT response due to irradiation over the 10-year life-time of the experiment is anticipated to be less than $10 \%$, which is deemed acceptable. Good correlation has been shown between the anode response of production VPTs (expressed as a light yield) measured in a magnetic field of $1.8 \mathrm{~T}$ (at angular orientations $-30^{\circ}<\theta<+30^{\circ}$ ) under pulsed light and the VPT net gain in zero magnetic field measured by the producer under continuous light. A sub-sample is also scrutinized in a magnetic field of $4 \mathrm{~T}\left(\theta=15^{\circ}\right)$ with an additional faceplate irradiation. The measured performance has matched the endcap design objectives.

\subsection{Crystal transparency monitoring}

Variation in the light transmission of the crystals is expected at the LHC due to radiation damage and recovery, which leads to cyclic transparency behavior between LHC collision runs and machine refills. The damage has been shown to be the creation of color centers, which then partly disappear due to self annealing. The scintillating process itself is not affected. During few hours of operation with colliding beams in the high luminosity regime, the response of crystals is expected to drop by a few percent in the barrel and by tens of percent in the endcaps. Therefore an optical monitoring system consisting of a laser source and an optical fiber distribution system has been developed to obtain short-term corrections of the measured energies of physics events and keep the intercalibration of the crystals within a precision of $0.5 \%$.

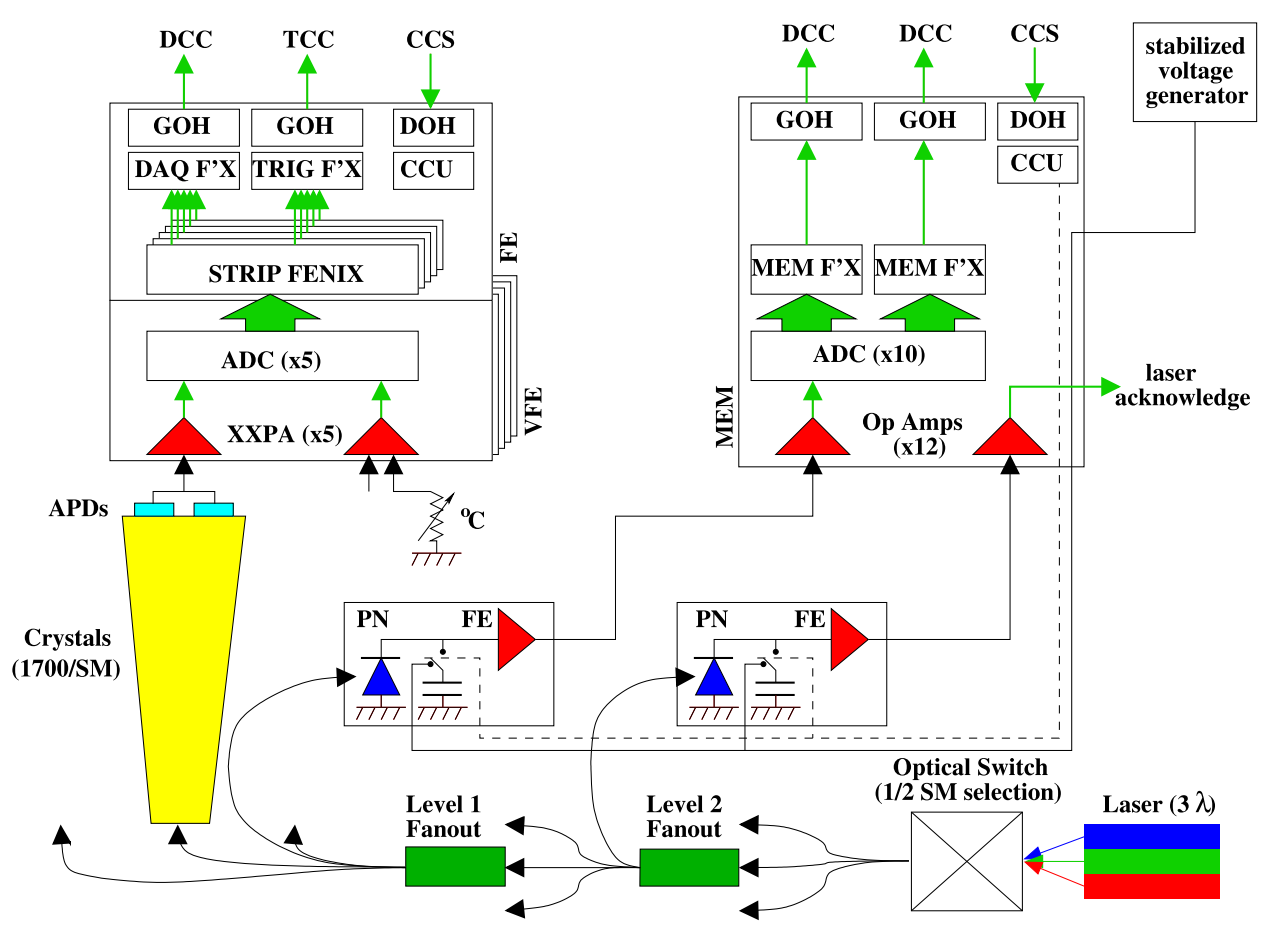

Figure 2.10: A schematic overview of the CMS ECAL light monitoring system.

The system consists of two lasers operating at four different wavelengths, 440 (blue), 495 (green), 709 (red), and $796 \mathrm{~nm}$ (infrared) and one spare laser with 440 (blue) and $495 \mathrm{~nm}$ (green), which distribute the light through optical fibers system. The blue light, which is close to the scintillation emission peak of the crystals, was chosen as the monitoring light. The infrared light, 
which is in a spectral range where the radiation induced changes and consequently the changes in transparency are negligible, is used to independently monitor the gain variations of the readout electronics chain. Light pulses from the laser source are sent by a fiber optic switch to one of the 88 calorimeter regions, i.e. 72 half supermodules and 16 regimes in the endcaps ${ }^{5}$.Then the laser pulses are delivered by a two-stage distribution system mounted on the calorimeter region to each crystal, every LHC beam cycle providing continuous monitoring. PN diodes measure the amplitude of the laser pulses as received by groups of 200 crystals, and provide a normalization of the magnitude of the injected light pulse. The monitoring system, including PN diodes and fibers, is radiation hard for doses up to those expected for 10 years of high luminosity LHC running. The system is described in detail elsewhere $[26,27]$ and a schematic overview is shown in Fig. 2.10.
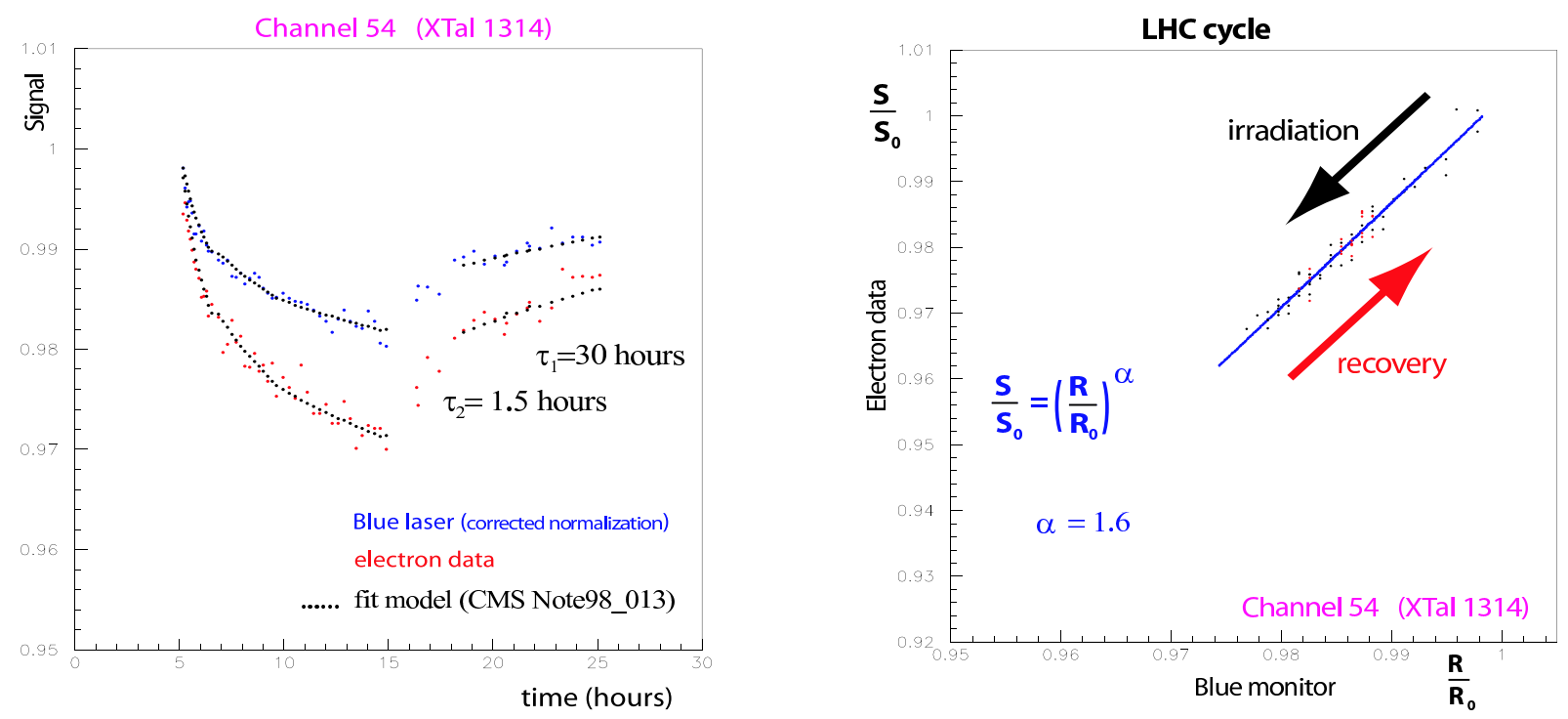

Figure 2.11: Left: The evolution of electron and laser responses as a function of time. Right: Crystal response to electrons $\left(\mathrm{S} / \mathrm{S}_{0}\right)$ versus response to laser light injected $\left(\mathrm{R} / \mathrm{R}_{0}\right)$ during irradiation and recovery phases $[28]$.

Because of the different optical paths and spectra of the injected laser pulses and the scintillation light, the change in response to the laser light is not equal to the change in response to scintillation light. An example of such a behavior is shown in Fig. 2.11 left [28], where one crystal was monitored for 10 hours during the irradiation and recovery periods. The electron response decreases much faster than the laser response as the dose increases (a dose rate of $0.25 \mathrm{~Gy} \cdot \mathrm{h}^{-1}$, same as the maximum dose rate in the barrel at high luminosity). The relation between the changes during the irradiation and recovery, which is displayed in Fig. 2.11 right,

\footnotetext{
${ }^{5}$ In situ, although there are 88 (72 for barrel and 16 for endcaps) light monitoring regions, rather than these 88 regions, 92 (72 for barrel and 20 for endcaps) values are readout. The reason is that for the endcaps there are 2 light monitoring regions which are readout by the 2 Data Concetrator Cards (DCCs).
} 
can be expressed by the power law:

$$
\frac{S(t)}{S\left(t_{0}\right)}=\left[\frac{R(t)}{R\left(t_{0}\right)}\right]^{\alpha},
$$

where $S(t) / S\left(t_{0}\right)$ and $R(t) / R\left(t_{0}\right)$ are the variations of the response to scintillation and laser light, respectively. A value $R(t)=A P D(t) / P N(t)$ is extracted for each crystal every 30 minutes that are needed to scan the whole ECAL. The Eq. 2.9 is determined from radiation damage models and for attenuation less than $10 \%$ describes well the behavior of all the crystals that have been evaluated during test beams, and it is expected to be valid in the ECAL for both low and high luminosity at LHC.

The parameter $\alpha$ was measured, for both crystal manufacturers (BTCP and SIC, see Chap.2.4) in the test beam during the last several years for the barrel as well as for the endcap. In the case of the barrel, the parameter $\alpha$ was found to be 1.53 (1.0) with a dispersion of $5 \%$ (10\%) [5] for crystal produced by BTCP (SIC) [29]. In the case of the endcap, the $\alpha$ values were 1.53 (1.1) with a dispersion of $20 \%(30 \%)$.

It was assumed that for the light transparency corrections only two values of $\alpha$ would be used and applied according to the crystal manufacturer (one value for crystals manufactured by BTCP and one for SIC crystals). However, due to the unexpected dispersion in $\alpha$ among measured crystals and very limited number of crystals for which the parameter was measured, the individual $\alpha$ value needs to be determined for each crystal. This can be done only in situ with physics events. The proposed iterative method for fast and accurate $\alpha$ extraction is based on the minimization of energy resolution with respect to the $\alpha$ value, and is currently under study [29].

The long term stability of the system in a real environment (temperature, high voltage, etc.) was verified during the test beam and is around $0.1 \%$ monitored for 500 channels over a period of 450 hours. This shows that the radiation induced transparency change can be monitored with a precision of typically $0.1 \%$ over several hundred hours [30]. The functionality of the system has been extensively tested for the whole barrel during the present commissioning phase.

\subsection{Conclusion}

The main mechanical parts and construction solutions of the electromagnetic calorimeter have been presented in this chapter. The conception of ECAL needs to stand for very hostile, but also very challenging conditions from an engineering point of view. The huge effort taken to build the hermetic and robust calorimeter with the desired energy resolution is in its finishing phase. Almost all parts of ECAL have been installed in situ. The barrel has been completed, all the 36 supermodules have been mounted at their final position with services that are fully functional and under control. The first cosmic events have been already collected and analyzed. The endcaps, which are still at the assembly center, will be added together with the preshower detector during the year 2008. The ECAL read-out electronics will be discussed in the following chapter. 



\section{Bibliography}

[1] CMS Collaboration, The CMS electromagnetic calorimeter project - Technical Design Report, ser. Technical Design Report CMS. Geneva: CERN, 1997, no. CERN/LHCC 97-33.

[2] E. Auffray et al., "Crystal conditioning for high-energy physics detectors," Nucl. Instrum. Methods Phys. Res., A, vol. 486, no. 1-2, pp. 22-34, 2002.

[3] P. Adzic et al., "Results of the first performance tests of the CMS electromagnetic calorimeter," CERN, Geneva, Tech. Rep. CMS-NOTE-2005-020, Oct 2005.

[4] E. Tournefier, "The preshower detector of CMS at LHC," Nucl. Instrum. Methods Phys. Res., A, vol. 461, no. 1-3, pp. 355-60, 2001.

[5] CMS Collaboration, CMS Physics: Technical Design Report - Detector performance and software, ser. Technical Design Report CMS. Geneva: CERN, 2006, no. CERN/LHCC 2006-001.

[6] CMS ECAL Preshower and Endcap Engineering Design Review v.2 - Preshower, CERN. Geneva: CERN, 2000.

[7] P. Aspell et al., "Results from the 1999 beam test of a preshower prototype," CERN, Geneva, Tech. Rep. CMS-NOTE-2000-001, Jan 2000.

[8] A. Annenkov et al., "Suppression of the radiation damage in lead tungstate scintillation crystal," Nucl. Instrum. Methods Phys. Res., A, vol. 426, no. 2-3, pp. 486-490, 1999.

[9] Z. R. Yuan, "Radiation damage in scintillating crystals," Nucl. Instrum. Methods Phys. Res., A, vol. 413, pp. 297-311, 1998.

[10] L. Zhang et al., "Performance of the monitoring light source for the CMS lead tungstate crystal calorimeter," IEEE Transcations on Nuclear Science, vol. 52, no. 4, pp. 1123-1130, 2005.

[11] CMS Collaboration, The Compact Muon Solenoid - Technical Proposal, ser. Technical Design Report CMS. Geneva: CERN, 1994, no. CERN/LHCC 94-38.

[12] P. Lecoq et al., "Lead tungstate $\left(\mathrm{PbWO}_{4}\right)$ scintillators for LHC em-calorimetry," Nucl. Instrum. Methods Phys. Res., A, vol. 365, no. 2-3, pp. 291-298, Dec 1994.

[13] A. N. Annenkov, M. V. Korzhik, and P. Lecoq, "Lead tungstate scintillator for high energy physics applications," IEEE Transcations on Nuclear Science, vol. 1, pp. 46-51, 1998. 
[14] E. Auffray et al., "Status of PWO crystal production for the electromagnetic calorimeter of CMS and of its construction," Nucl. Instrum. Methods Phys. Res., A, vol. 537, pp. 373-378, 2005 .

[15] S. Baccaro et al., "An automatic device for the quality control of large-scale crystal's production," Nucl. Instrum. Methods Phys. Res., A, vol. 459, no. 1-2, pp. 278-84, 2001.

[16] L. M. Barone et al., "REDACLE database for the workflow management of the CMS ECAL construction," CERN, Geneva, Tech. Rep. CMS-NOTE-2003-022, Aug 2003.

[17] "CRISTAL project," http://proj-cristal.web.cern.ch/proj\%2Dcristal/.

[18] E. Longo, "Avalanche photodiodes for the CMS electromagnetic calorimeter," CERN, Geneva, Tech. Rep. CMS-CR-1998-002, Jan 1998.

[19] "CMS ECAL APD site," http://cms-ecal-apd.web.cern.ch/cms-ecal-apd.

[20] Z. Antunovic et al., "Radiation hard avalanche photodiodes for the CMS detector," Nucl. Instrum. Methods Phys. Res., A, vol. 537, pp. 379-382, 2005.

[21] D. Bailleux et al., "Hamamatsu APD for CMS ECAL: Quality insurance," Nucl. Instrum. Methods Phys. Res., A, vol. 518, pp. 622-625, 2004.

[22] P. Depasse et al., "A validation program for the CMS ECAL photodetection capsules," CERN, Geneva, Tech. Rep. CMS IN-2000/013, Feb 2000.

[23] — - "Final calibration results of CMS electromagnetic calorimeter photodetectors," Nucl. Instrum. Methods Phys. Res., A, vol. 567, pp. 242-245, 2006.

[24] N. A. Bajanov et al., "Fine-mesh photodetectors for CMS endcap electromagnetic calorimeter," Nucl. Instrum. Methods Phys. Res., A, vol. 442, no. 1-3, pp. 146-9, 2000.

[25] Y. I. Gusev et al., "Super radiation hard vacuum phototriodes for the CMS endcap ECAL," Nucl. Instrum. Methods Phys. Res., A, vol. 535, pp. 511-516, 2004.

[26] M. Anfrevilleb et al., "Laser monitoring system for the CMS lead tungstate crystal calorimeter," CERN, Geneva, Tech. Rep. CMS-NOTE-2007-028. CERN-CMS-NOTE-2007-028, Nov 2007.

[27] D. Bailleux et al., "Performance of the monitoring light source for the CMS lead tungstate crystal calorimeter," CERN, Geneva, Tech. Rep. CMS-CR-2005-001. CERN-CMS-CR-2005001, Jan 2005.

[28] A. Van Lysebetten and P. Verrecchia, "Performance and measurements of the light monitoring system for CMS-ECAL from 2002 test beam data," CERN, Geneva, Tech. Rep. CMS-RN-2004-001, Feb 2004.

[29] C. Rogan, Talks presented at ECAL detector performance and test beam meetings, http://indico.cern.ch/contributionDisplay.py?contribId $=2 \&$ confId $=24835$, http://indico.cern.ch/contributionDisplay.py?contribId=1\&confId=23924, http://indico.cern.ch/contributionDisplay.py?contribId=6\&confId $=22391$, http://indico.cern.ch/contributionDisplay.py?contribId=3\&confId=21266, 2007.

[30] A. Bornheim, "The CMS ECAL laser monitoring system," in XII International conference on Calorimetry in high energy physics - CALOR 2006, Chicago, USA, 2006. 


\section{Chapter 3}

\section{Readout electronics}

\subsection{Introduction}

High requirements are put on the ECAL readout electronics in order to fulfill demanding calorimeter physics performances. The electronics must be very fast to match a $25 \mathrm{~ns}$ LHC bunch crossing rate and provide an energy measurement with high precision over a large dynamic range from about $50 \mathrm{MeV}$ to several $\mathrm{TeV}$ corresponding to 16 bit in digitization. Moreover, an electronic noise must be kept less than $\sim 50 \mathrm{MeV}$ per channel. The final electronics architecture $[1,2]$ was defined in 2002 when it was decided to move, in contrary to original ECAL readout electronics strategy, the computation of trigger primitives and the primary event buffers on a front-end level. This decision was driven mostly by high cost of optical data links and by new development of radiation hard Application Specific Integrated Circuits (ASICs) based on $0.25 \mu \mathrm{m}$ technology that became price affordable and which allow to place a part of the readout electronics directly onto the calorimeter. Consequently, the number of the high-speed optical data links, which are in charge of communication between the front-end and the upper level, was decreased by about a factor of eight.

In this chapter, a short description of the ECAL read-out electronics is given. The first half is focused on the front-end read-out system (on-detector electronics), whereas the second part covers the main parts of the upper level electronics system (off-detector electronics).

\subsection{Electronics system layout}

The ECAL electronics can be described as two subsystems: The on-detector electronics located directly inside the calorimeter just behind the crystals and the upper level readout system, so called off-detector electronics, located outside the experimental area. Both systems communicate through 12,500 high-speed optical data links.

The scintillation light produced in a crystal by an electromagnetic shower is captured by a photodetector, converted into an electrical signal and processed by on-detector electronics. The signal is amplified and digitized in the Very-Front-End (VFE) card and then routed to the Front-End (FE) boards, the last step of the on-detector electronics. In the FE boards, the basic elements are partly computed allowing the identification of electromagnetic showers by the Level-1 calorimeter trigger, called trigger primitives. A second phase of the trigger primitive evaluation is realized by the Trigger Concentrator Card (TCC) which belongs to the off- detector subsystem. The crystal data are transmited from the FE board upon level-1 trigger accept signal to the Data Concentrator Card (DCC). The FE boards are connected to the off-detector 
electronics by serial optical links working at $800 \mathrm{Mbits}^{-1}$ over $90 \mathrm{~m}$.

\subsection{On-detector electronics}

Because of the very hostile detector environment the on-detector electronics circuits are designed to be radiation-hard and insensitive to a high magnetic field. In addition, due to the strong crystal and APD temperature dependence, the electronics must have low power consumption $(\sim 2 \mathrm{~W} /$ channel $)$ in order to minimized their thermal input to the system and thus allow the temperature to remain stabilized with a requesting precision of $0.1 \%$. The basic unit of the on-detector readout electronics is composed of groups of four dedicated boards reading and processing a $5 \times 5$ crystal array. In the barrel this corresponds to a trigger tower and in the endcaps to a super-crystal. Each trigger, schematically displayed in Fig. 3.1, is composed of five VFE cards, one FE card, one Low Voltage Regulator (LVR) card and one Motherboard (MB). The trigger tower is connected by two high speed $\left(800 \mathrm{MBit} \mathrm{s}^{-1}\right)$ optical links transferring trigger at $40 \mathrm{MHz}$ and data at $100 \mathrm{kHz}$, and one link (not seen in Fig. 3.1) providing the clock and control.

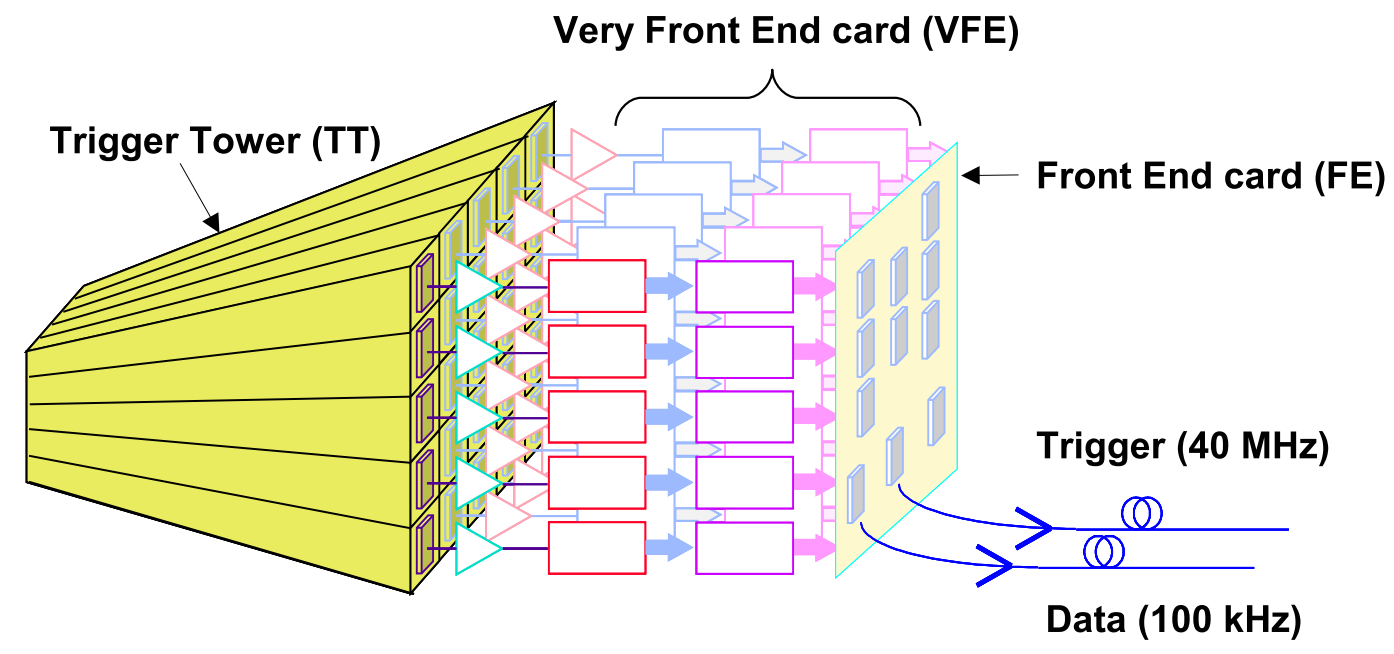

Figure 3.1: Schematic view of one barrel trigger tower as a basic block of the on-detector readout electronics reading and processing a $5 \times 5$ crystal array.

\subsubsection{Motherboard}

The entirely passive motherboards are located beneath the cooling system for the electronics and are used to route the signals from the photodetectors to the VFE cards (the motherboards also arrange the VFE cards along $\phi$ orientation, the submodules having connectors along $\eta$ ), to filter and distribute high voltage to the photodetectors, to distribute low analog voltage to the VFE cards from LVR board, and to provide a ground connection to the grid. Each supermodule contains 68 motherboards which each connect to the photodetectors via kapton flexible-print cables. In addition, signals for temperature monitoring thermistors are routed from the sensor to the VFE cards. 


\subsubsection{Low voltage regulator board (LVR)}

The LVR cards $[3,4]$, which supply the low voltage power to the complete front-end system, are connected through a Low Voltage Distribution (LVD) panel directly to the external Low Voltage Power Supplies (LVPS) sitting in the CMS racks attached to the outside of the CMS iron yoke, more than $20 \mathrm{~m}$ from the calorimeter. There are in total 772 LVPS needed for the whole ECAL (612 for the barrel and $\sim 160$ for both endcaps), whereas one LVPS supplies 100 readout channels. Each LVPS and LVD serve four trigger towers or super-crystals, i.e. provide the power and voltages to the four LVR boards.

The LVR board houses the radiation-hard low voltage regulators LHC4913PDU ${ }^{1}$ [5] and three Detector Control Units (DCUs) monitoring output and input voltages as well as the card temperature. The LHC4913PDU regulator is a positive voltage regulator with an inhibit function which has a maximum output current of $3 \mathrm{~A}$, a load dependent drop-out voltage of $0.5 \mathrm{~V}$ per ampere of output current, an input voltage range from 3 to $12 \mathrm{~V}$, and four fixed output voltages between 2.5 and $8 \mathrm{~V}$. Moreover, it has several protective elements such us an adjustable output current limitation protecting the outputs from damaging short-circuits, over-current, and overtemperature protection.

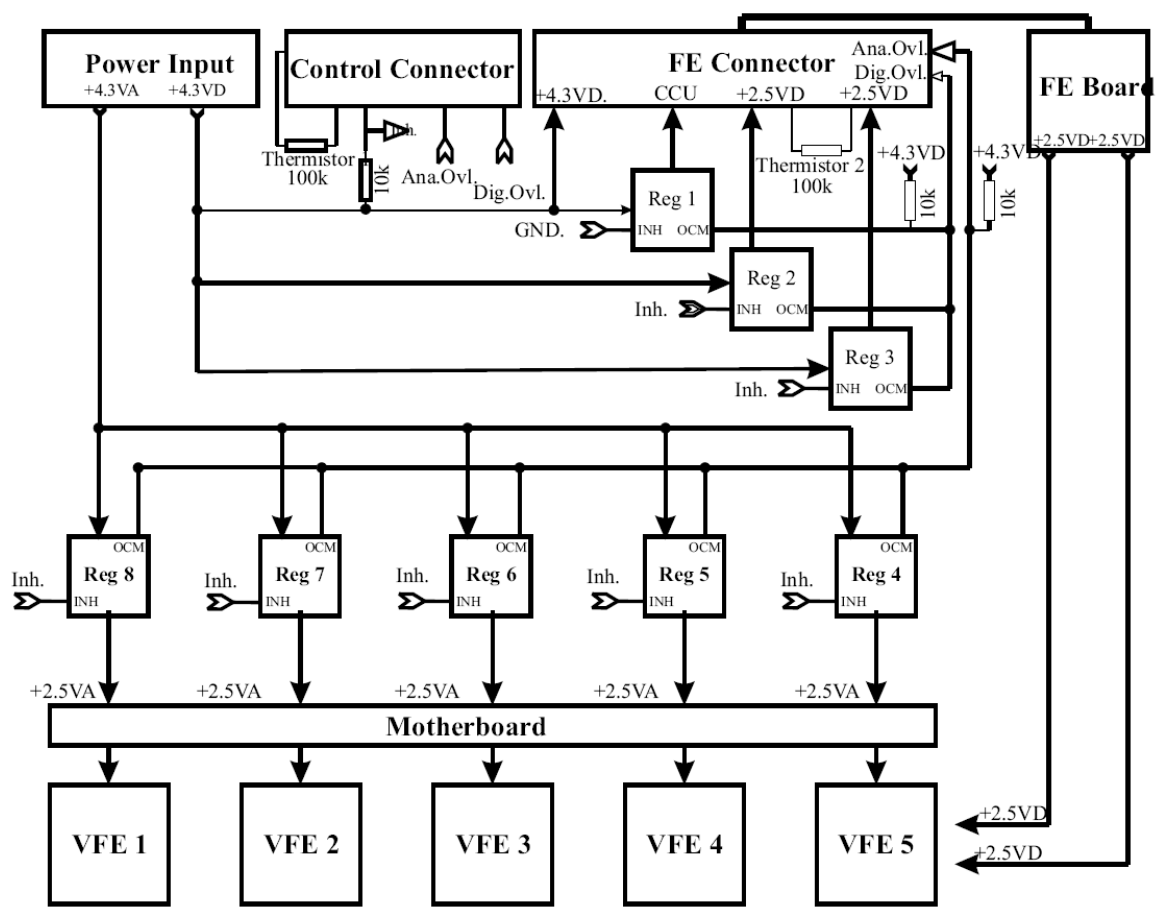

Figure 3.2: Schematic view of the Low Voltage Regulator (LVR) prototype architecture powering five VFE boards and one FE board.

In Fig. 3.2 [3] a schematic drawing of the LVR prototype architecture with eight regulators, a power pipeline, and voltages to the frond-end electronics through the MB and FE is shown. Five

${ }^{1}$ Developed by the CERN/ST microelectronics. 
regulators connected in parallel provide regulated $+2.5 \mathrm{~V}$ analog voltages to the $5 \mathrm{VFE}$ cards distributed via the motherboard and two others provide digital voltages $+2.5 \mathrm{~V}$ to the VFE boards and FE board. The last regulator powers the clock and control system including the token ring board. Its inhibit switch, which is connected to the ground, is turned ON whenever the input power is provided. The inhibit of all other regulators is controlled by LVPS. This allows the power for each trigger tower to be switched ON or OFF individually by the LVPS without affecting the other trigger towers functionality.

In order to achieve required stability of the pedestal (1/10 of a ADC count), the contribution of the low voltage power supply to the noise at the ADC input must be about $40 \mu \mathrm{V}$. Considering that the MGPA power supply rejection ratio PSRR, which describes the amount of noise from a power supply that the MGPA can reject, is better than a factor of 10 and hence the LVR voltage have to be stable within $400 \mu \mathrm{V}$. The stability of the LVPS is required to be about $40 \mathrm{mV}$. For one trigger tower a total current and power consumption is estimated to be $\sim 16 \mathrm{~A}$ and $\sim 68.5 \mathrm{~W}(\sim 2.7 \mathrm{~W}$ per channel $)$, respectively. Approximately same values are expected for a endcap super-crystal. About $46 \%$ of this power is dissipated in the low voltage regulators.

\subsubsection{Very-front-end board (VFE)}

The VFE board $[6,7]$ comprises five identical and independent read-out channels, which shape, amplify and digitalize signals coming from five crystals. In the original readout electronics design, the photodetector signal was processed by a unique integrated circuit called FPPA [8] (Floating Point Pre-Amplifier) which integrates a multi-gain transimpedance amplifier with internal shaping and four-range amplification stages (1, 5, 9, and 33), and an analog multiplexer selecting the gains and multiplexing them to the ADC. Due to the level of electronics noise, higher than that deemed acceptable, it was considered safer to develop an alternative ASIC providing similar functionality - Multi-Gain Pre-Amplifier (MGPA).

Therefore, in the final solution, each channel consists of the MGPA [9] with three different parallel gain stages (1, 6, and 12), a 12 bit multi-channel Analogue-to-Digital Convertor (ADC) AD41240 [10] with an internal logic to choose the highest non-saturated gain as the ADC output, and two level adapters LVDS-RX to transform the low voltage differential signal outputs of the ADC to the single ended inputs of the FE boards and distribute the $40 \mathrm{MHz}$ LHC clock. This configuration, which implements an analog dynamic range compression by digital gain selection (i.e. the gain selection is performed after digitization), allows the dynamic range to expand, keeping the same resolution and speed as a 15 bit ADC which was too expensive. In addition, the barrel VFE board also incorporates a Detector Control Unit (DCU) chip for measuring the APD leakage current and the crystal temperature. A photograph of the VFE card showing five independent channels with the MGPA, ADC chips, and two LVDS-RX buffers is seen in Fig. 3.3.

\subsubsection{Front-end board (FE)}

The FE board $[11,12]$ is a part of the front-end system and serves one trigger tower or supercrystal. The board sits transversely to the LVR and VFE boards (see Fig. 3.1) and is connected with them by two-row connectors located on its back side as is seen in Fig. 3.4 right. From the LVR card, the board receives the regulated power for its internal uses and for distributions to the digital parts of the VFE boards. In addition, the FE board also provides the clock and control to the VFE cards. On the other side, shown in Fig. 3.4 left, all the ASICs, providing the main functionality of the board, as well as connectors to data links and to the token links are placed. 


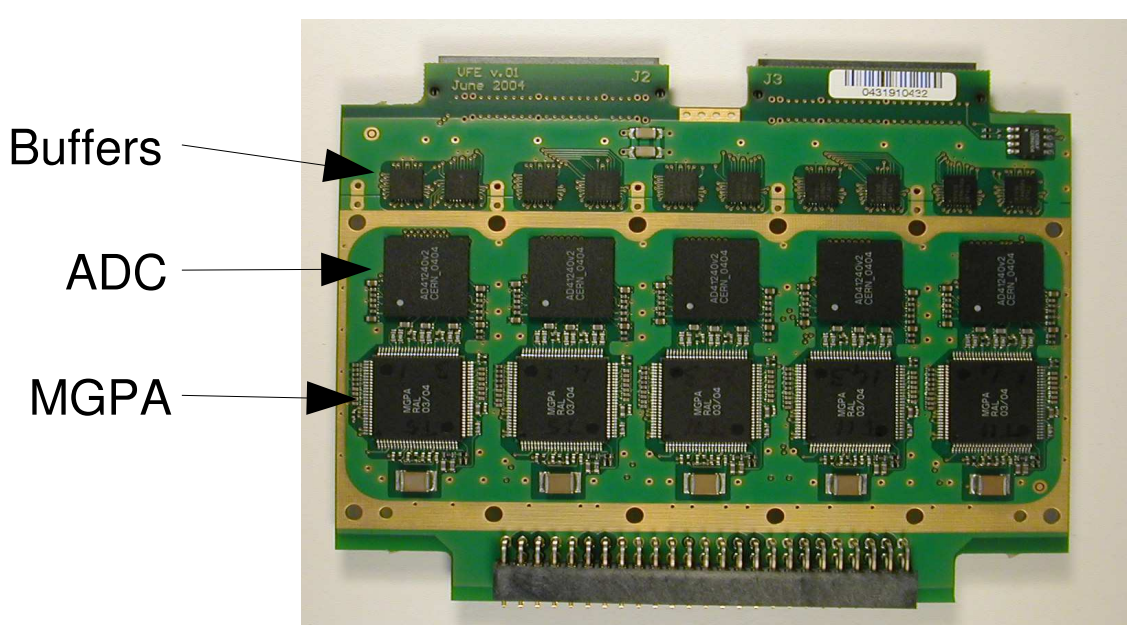

Figure 3.3: Photograph of the Very-Front-End (VFE) card showing the five channels with MGPA, ADC, and two buffers each.
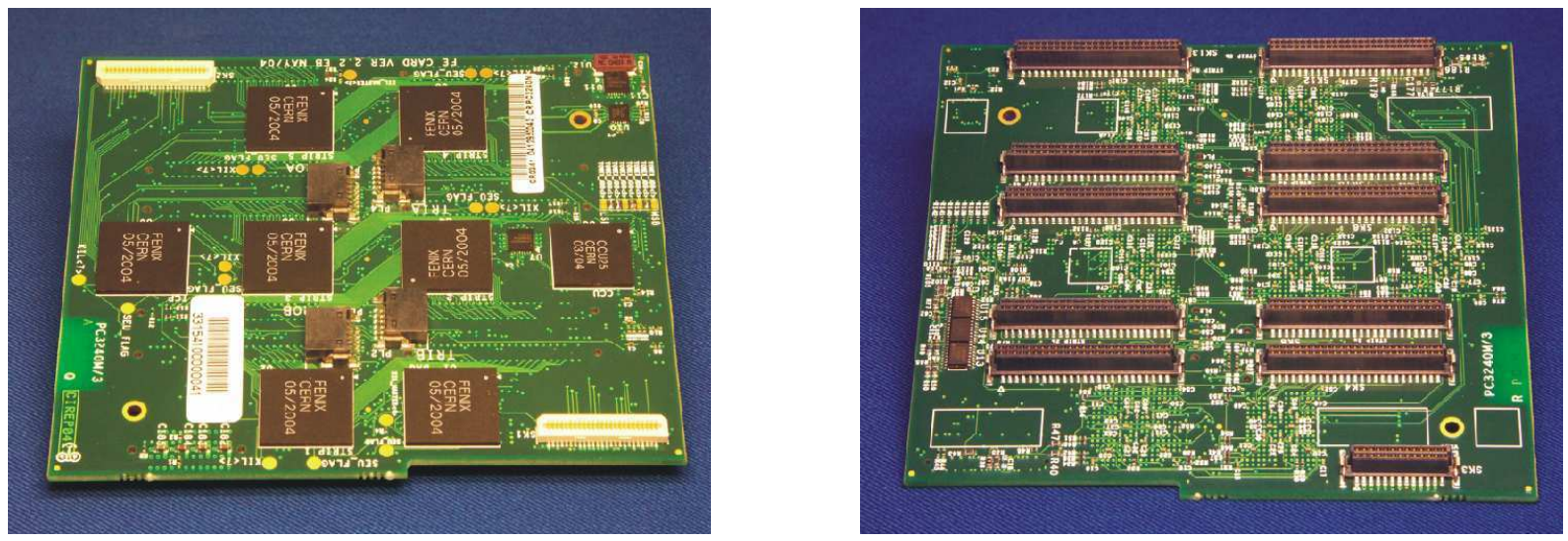

Figure 3.4: Left: Photograph of the barrel FE board front side showing 7 FENIX chips, CCU chip and two GOHs connectors. Right: Back side of the barrel FE board with connectors for five VFE boards (five $\times 2$ connectors) and one for LVR board. 
The principal tasks of the FE board are to generate the trigger primitives for the Level1 trigger processor and to provide the digital pipeline and the primary event buffers. These functionalities are implemented in seven dedicated FENIX ASICs, which provide four different functions within a single chip. The outputs of the FE board correspond to 1 optical link to the DCC (Data path) and 1 or 5 optical links to the TCC (trigger path) in the barrel or the endcaps, respectively. It is obvious that this configuration requires high quality clock and control systems, which were taken over from the well-developed system of the CMS tracker project.

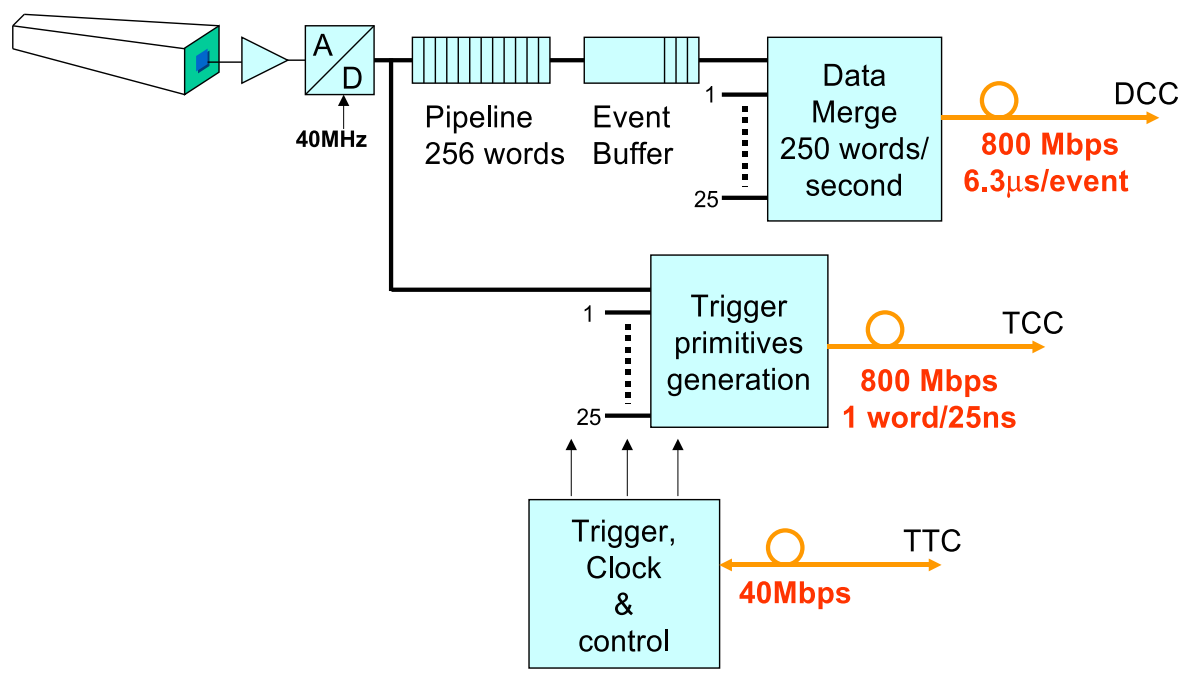

Figure 3.5: Schematic block diagram of the ECAL on-detector readout electronics system.

\section{Readout procedure}

The readout of the data for a physics event satisfying the Level-1 trigger requirements is ilustrated in Fig. 3.5 and carried out in the following three steps: First, the FE board receives the digital data (16 bits per channel at $40 \mathrm{MHz}$ ) from the output of five VFE cards and stores them temporarily into a digital pipeline while the trigger primitive ${ }^{2}[13]$ is calculated for each bunch crossing and, after the conversion into a serial optical signal via the Giga-bit Optical Link (GOL) [14], is sent by a trigger link to the Trigger Concentrator Card (TCC) belonging to the off-detector system. The pipeline capacity is programmable to correspond exactly to the Level-1 trigger latency which can last at most $3.2 \mu \mathrm{s}$ (128 bunch crossings). Second, when the board receives a Level-1 trigger accept signal coming back through the TCC system and through the Control and Clock System (CCS), a time frame of 10 samples for every channel is transferred from the pipeline to the primary event buffers having a capacity of 25 events. Finally, as soon as the readout data link is available, the data for all the 25 channels, together with an event identification and a trailer word, are formatted and transmitted through the GOL data link to the off-detector Data Concentrator Card (DCC) making them accessible for the

\footnotetext{
${ }^{2}$ Trigger primitive comprises an evaluation of the transverse energy $E_{T}$ deposited in a trigger tower and the fine grain veto bit in the barrel and five partial sums of five crystals in the endcaps, and the bunch crossing assignment.
} 
data acquisition (DAQ) system. The readout service time is $7.15 \mu \mathrm{s}$ (286 bunch crossings) and the overflow probability is in order of $10^{-8}$.

\section{FENIX}

As mentioned before the main functionality of the $\mathrm{FE}$ board is engaged in a single multipurpose radiation-hard ASIC named FENIX $[15,16]$ (Front-End New Intermediate data eXtractor) which performs complex digital data treatment of data coming from the VFE cards. Although only one version of the chip has been manufactured for the ECAL, it can work in four different operation modes: Strip ${ }^{3}$, TCP, DAQ, and MEM. The mode is chosen by two external pins and therefore it could not be changed by programing. There are seven FENIX chips placed on the barrel version of the FE board and six on the endcap board as is shown on the block diagrams in Fig. 3.6. In the barrel, the processed data flow, together with appropriate header and trailer containing channel identification, is split into a trigger primitive generation path controlled by FENIX running in TCP mode, and to a data readout path operated by DAQ FENIX, see Fig. 3.6 left. In the endcap, the TCP FENIX is not present and data are sent through the GOL to the TCC board, see Fig. 3.6 right. The functionality of each operating mode is described below.
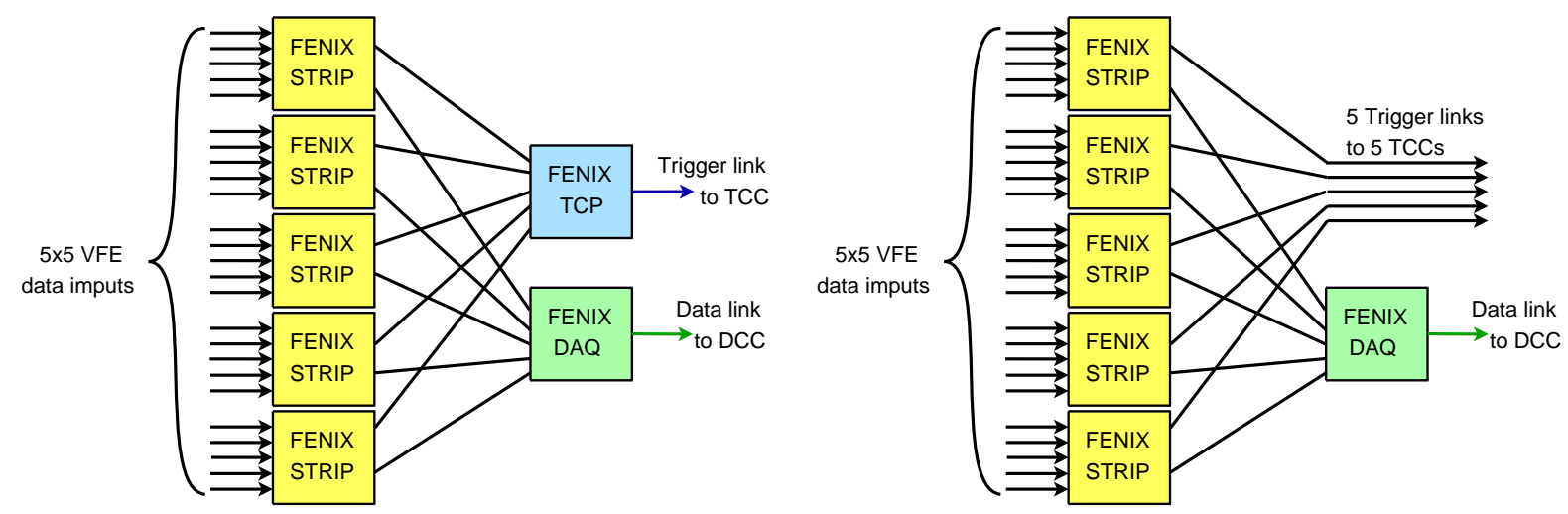

Figure 3.6: Block diagrams of the FENIX chips with indication of their operation modes for the barrel (left) and the endcap (right) versions of the FE board.

Strip mode - Five FENIX chips on the FE board are operated in the Strip mode whereas each chip processes digitalized output from five channels of the VFE card. It computes a total transverse energy $E_{T}$ deposited in one crystal strip in $\phi$ geometry which is needed for the trigger primitive generation and to perform the bunch-crossing assignment. Moreover, the chip also contains the pipeline and the primary event buffers for all five channels. The procedure of computation of the $E_{T}$ starts in a lineariser which reconstructs a signal amplitude from 10 consecutive samples, applies necessary calibration constants and multiplies the signal by a factor $\sin \left(\theta_{\eta}\right)$ in order to determine the appropriate value of the $E_{T}$. This

\footnotetext{
${ }^{3}$ A strip is defined as a five channels (with same $\phi$ geometry in the barral and a more complicated geometry in the endcaps) in one trigger tower. Thus one trigger tower consists of five strips. The signal in one strip is procesed by one VFE card.
} 
part is processed in parallel for each VFE channel. After linearization all five signals are added together and the obtained linearized amplitude is proportional to the deposited energy in one crystal strip. Then the signal is filtered by a finite impulse response (FIR) filter which determines the pulse amplitude using weights delivered from a pulse model. Finally, a peak finder is applied in order to perform the bench crossing assignment [13]. It is clear that the computation of the $E_{T}$ requires the coarse absolute calibration of each VFE channel.

TCP mode - The FENIX chip working in the Trigger Cell Processor - TCP mode is present only in barrel $\mathrm{FE}$ boards, where trigger array $(5 \times 5$ crystals $)$ corresponds physically to one trigger tower ( 5 strips). The TCP FENIX gathers data from the five Strip FENIX chips and finalizes the trigger primitive, which includes the computation of total the energy $E_{T}^{\text {tot }}$ deposited in the whole barrel trigger tower and it also generates a Fine Grain Veto (FGV) bit, which estimates the lateral extension of the electromagnetic shower. The FGV bit is determined by comparison of the maximum energy deposited in two adjacent strips $E_{2}^{\max }$ with $E_{T}^{\text {tot }} \times H T(L T)$, where $L T$ and $H T$ are programmable low and high thresholds, respectively. Finally, the chip is responsible for encoding the trigger primitives and sending them at $40 \mathrm{MHz}$ by a high-speed optical link $\left(800 \mathrm{Mbit}^{-1}\right)$ to the TCC board.

$D A Q$ mode - The FENIX chip running in DAQ mode controls the readout of five FENIX chips operating in the Strip mode and encapsulates the event by adding a trigger tower ID, an event ID, a bunch crossing ID, and a Cyclic Redundancy Check (CRC) in the trailer word. The channels are read and sent to via GOL by an optical link to the off-detector DCC board upon Level-1 trigger accept signal.

MEM mode - The FENIX chip operating in the Monitoring Electronic Module - MEM mode is a mixture between the Strip and DAQ modes and it is used for the readout of digital data of the laser monitoring injection system (see Chap. 2.6).

Although the FENIX chip is based on the radiation tolerant technology, intrinsically it is not protected against the Single Event Up-set (SEU), which is caused by high-energy particle striking to a sensitive node of the chip. The SEU then stands for any error generated on the output or in chip operation. In order to cope with the SEU, the triple redundant techniques have been implemented for the configuration and finite state machine registers, see Fig. 3.7. The Error Correction Code (ECC) has been applied on any data stored in FENIX memories by using a Hamming code [17] after separation of the 16 bit data into two 8 bit words. Hence a single-bit errors can be corrected and double-bit errors can be detected. In addition the Digital Signal Processing (DSP) blocks are doubled and their outputs are compared in order to detect SEU as well as calculation errors. A functionality of the implemented protections has been tested, on a complete front-end at PSI protons source for doses expected for 10 years of LHC operation. The tests have given satisfactory results verifying the robustness against the SEU [16].

The settings of the internal registers of the FENIX as well as the control of the FE board are done via a Token Ring. Reception and decoding of the control parameters is done in a rad-hard ASIC called Communication and Control Unit (CCU). 

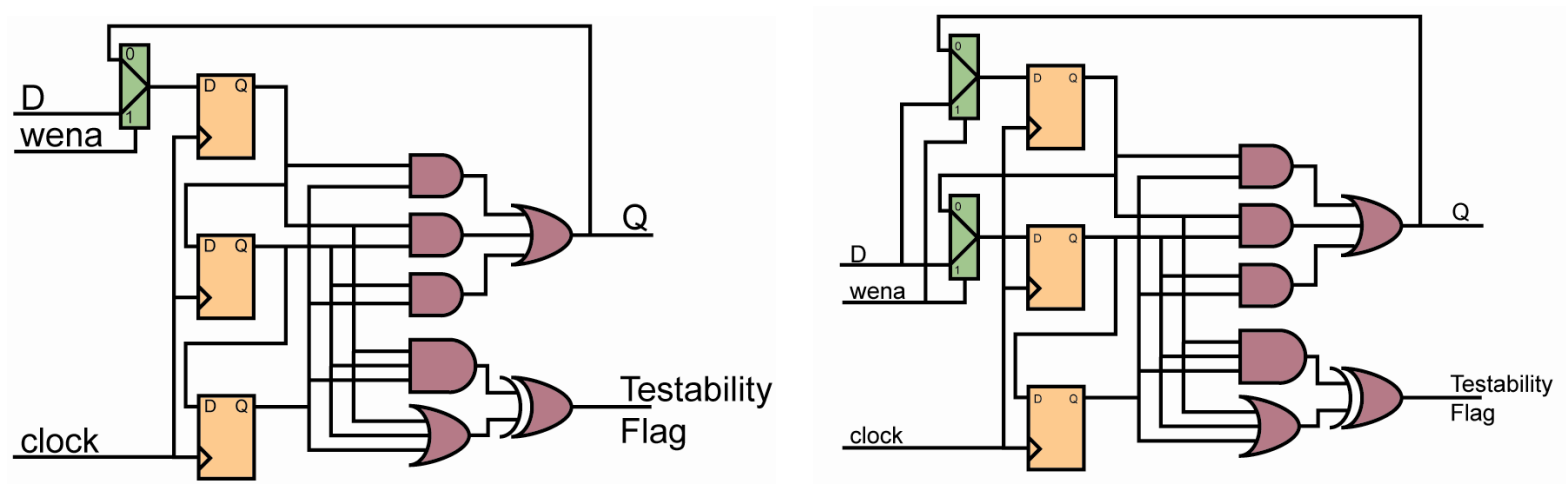

Figure 3.7: Left: Triple redundant configuration register. Right: Triple redundant Finite State Machine register. Both are implemented in the FENIX chips in order to protect against the SEU.

\section{Clock and control}

The FE board hosts the Communication and Control Unit (CCU) type CCU25, the Phase Locked Loop (PLL) and the Crystal Oscillator Phase Locked Loop (QPLL) ${ }^{4}$ which were both originally developed for the CMS tracker detector.

The CCU $[18,19]$ is intended for the clock distribution as well as for monitoring and slow control. It has dual network input and output ports allowing the cabling of the redundant network. Therefore the communication with the CCU can be done via a dual electrical ring controlled through a Token Ring Board (TRB) by the off-detector mezzanine Frond-End Controller (mFEC) placed on the CCS card. The CCU contains several types of peripheral controllers providing interface to the various front-end chips. Its sixteen $\mathrm{I}^{2} \mathrm{C}$ master ports are used for slow control and monitoring of all the FENIX chips, VFE boards, GOL (DAQ and trigger links) as well as for DCU of the LVR and PPL. Four parallel I/O bus controllers are used mainly for monitoring of the optical links and the flags from low voltage regulators [15].

The PLL $[11,12]$ is in charge of the fast control which is distributed by the T1 signal encoded by means of missing rising edges along the global clock distributed via a token ring from the CCS. The signal T1 is detected and extracted by the PLL and, after its decoding implemented in each FENIX, provides $4+2$ broadcast commands listed in Tab. 3.1. The command Lv1 is linked with Level-1 trigger decision. When the signal arrives the data stored in the pipelines are sent for consecutive off-detector data processing. A local bunch crossing counter is the reference counter for the event builder, the BCO command is used to set this counter to 0 and hence stamps the beginning of the LHC orbit for the whole ECAL. The Re_synch signal is sent in order to reset all pointers in the system, i.e. clear all front-end data pipelines and error conditions (e.g. caused by SEUs). In principle, this signal is used for the system resynchronization. If the MGPA testpulse is needed in order to test the VFE electronics or for other purposes, such as the gain ratio determination for high energy signal reconstruction (see Chap. 6.7), the Force_VFE command is applied. By two consecutive T1 signals it is also possible to reset only the FENIX chip.

\footnotetext{
${ }^{4}$ In fact, the QPLL is a modified tracker Phase Locked Loop (PLL) in order to provide high quality clock for the fast optical link and for the high performance sampling of the VFE electronics.
} 


\begin{tabular}{|l|c|l|}
\hline \hline T1 pattern & Command & Description \\
\hline 100 & Lv1 & Readout request \\
101 & BCO & Sets a local bunch crossing counter \\
110 & Re_synch & Reset all state machines \\
111 & Force_VFE & Force gain and test pulse injection \\
$110110^{a}$ & Pwup_reset & Re-initialises FENIX ASIC \\
$1100110^{a}$ & Pwup_reset & Re-initialises FENIX ASIC \\
\hline \hline
\end{tabular}

${ }^{a}$ If two consecutive Pwup_reset broadcasts are received, a power-up reset is performed in the FENIX chip only.

Table 3.1: Fast control encoding of the synchronous broadcast signals.

\section{Gigabit optical Hybrid}

Data and trigger primitives are transferred by serial high speed optical links operating at 800 Mbit s$^{-1}$ to the DCC and TCC boards. Since these data are stored in FE pipelines in an electronic digital parallel form, the FE board employs an optical transmitter named Gigabit Optical Hybrid (GOH) [20], which is responsible for data serialization and their electronic-tooptical conversion. The FE board hosts two transmitters in the case of the barrel (one GOH for the trigger and one for the data links) or six of them in the case of the endcaps (five for the trigger and one for the data links). The GOH receives power, clock and control, and 16-bit parallel data at $40 \mathrm{MHz}$ from the FE board. The data are serialized and encoded by a Gigabit Optical Link $(\mathrm{GOL})^{5}[14,21]$ serializer and laser driver chip. Electrical signals generated by the laser driver are converted into optical signals by the laser diode, and then transmitted via a connected pigtail optical fiber to the distributed patch-panel. The GOL supports two encoding protocols: the G-Link and 8-bit/10-bit protocols, which are both used in the ECAL. The first one is required by the Agilent HDMP-1034A deserializer chip on the TCC board, which performs fast deserialization of the trigger data that is required by a strict time budget for the trigger data processing. The second one is needed for deserializer-encoded Xilinx FPGA on the DCC board, which performs slower deserialization in comparison with the Agilent, but adequate to the slower data path and affordable. The laser transmitter ${ }^{6}$ [22], originally designed for the CMS tracker detector, is an edge-emitting semiconductor diode housed in a custom-built laser-pill placed on the GOH board. Their working parameters $(\lambda=1310 \mathrm{~nm}$ and a rise time of $0.3 \mathrm{~ns})$ are fully compatible with $800 \mathrm{Mbit} \mathrm{s}^{-1}$ digital operation and for optical data transfer and receivers.

\subsubsection{Token ring board (TRB)}

The TRB [23] is the optical-to-electrical token ring converter and main node of the ECAL electrical token ring network which is created by the interconnection network among the CCUs placed on the FE boards providing distribution of the clock and the trigger commands delivered from the off-detector clock and control system. One token ring serves 8, 9, or $10 \mathrm{FE}$ boards, hence 8 TRB are needed for one supermodule. Since one broken node interrupts completely the functionality of the whole ring, the redundancy of the token ring system is needed.

\footnotetext{
${ }^{5}$ Developed by the CERN Microelectronics Group

${ }^{6}$ Developed by ST Microelectronics
} 


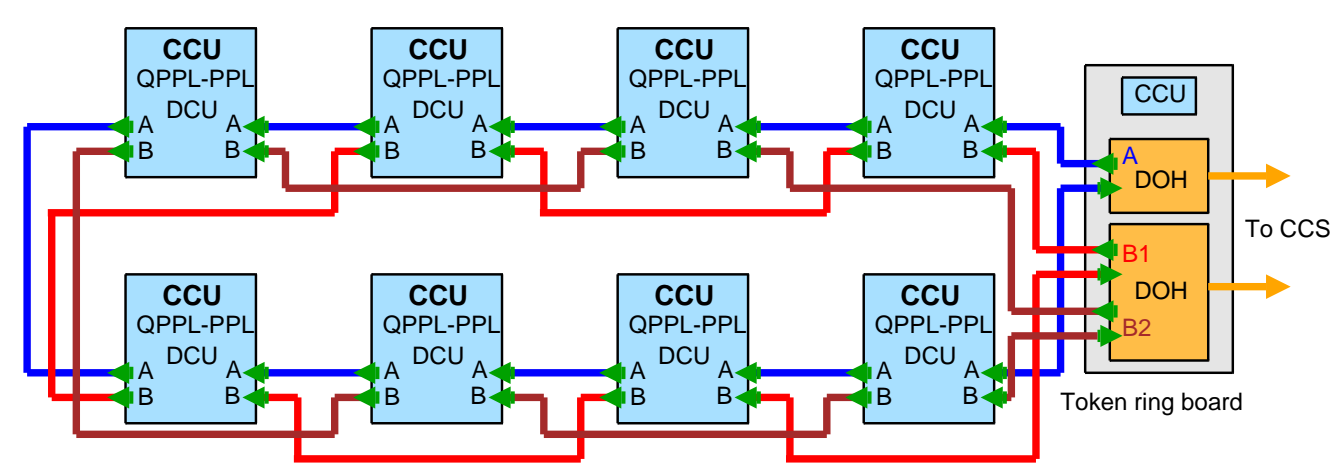

Figure 3.8: Schematic diagram of the token ring architecture serving eight FE boards. The token link board is also shown with $2 \times 2$ optical links to the off-detector CCS mFEC card.

The redundancy schema, as can be seen in Fig. 3.8, is ensured by doubling the signal paths that allow bypassing of a faulty CCU. Therefore, the TRB hosts two Digital OptoHybrids (DOHs), one for the primary and one for the secondary ring network. The DOH [24] is a two channel transceiver, working at $40 \mathrm{Mbits}^{-1}$, which interfaces the optical signals from the mFEC and the electrical signals from the TRB. One of two DOH channels is used for the clock and broadcast commands, and another transmits the control data. Hence the TRB is connected by four optical links to the mFEC card by which the whole token ring is controlled.

\subsection{Off-detector electronics}

The ECAL off-detector electronics $[2,25]$ is composed of four different electronic modules: the Control and Clock System (CCS), the Trigger Concentrator Card (TCC), the Data Concentrator Card (DCC), and the Selective Readout Processor (SRP). The first three modules are sitting in 18 VME-9U crates and the later is mounted in one VME-6U crate, all placed in the underground counting room connected with the on-detector electronics by around $90 \mathrm{~m}$ long high-speed optical links. A schematic view of the off-detector electronics with relations among them is displayed in Fig. 3.9.

The barrel trigger primitives or endcap strip sums are transmitted at $40 \mathrm{MHz}$ to the TCC card, where the trigger primitives are finalized and synchronized. Then the data are sent to the Regional Calorimeter Trigger (RCT), where detection of signatures for isolated and nonisolated $e / \gamma$, jets, $\tau$-leptons, and missing and total transverse energy is performed. The selected candidates along with energy sums are then forwarded via the Global Calorimeter Trigger (GCT) to the Global Trigger (GT) for further sorting [26]. The level-1 trigger accept signal, generated in the GT, is transmitted through the Timing Trigger and Control system (TTC) to the CCS board, which sends the signal to the on-detector electronics. On the signal reception the crystal data are formatted and transmitted to the DCC, where the integrity check and data volume reduction, based on the information given from the SRP, are performed. Finally, the formatted data are sent to the DAQ system using the S-link $64^{7}[27]$.

\footnotetext{
${ }^{7}$ S-link 64 is an extended 64-bit version of the S-link (Simple link interface), which is high-performance data acquisition standard developed at CERN for FIFO-like data links.
} 


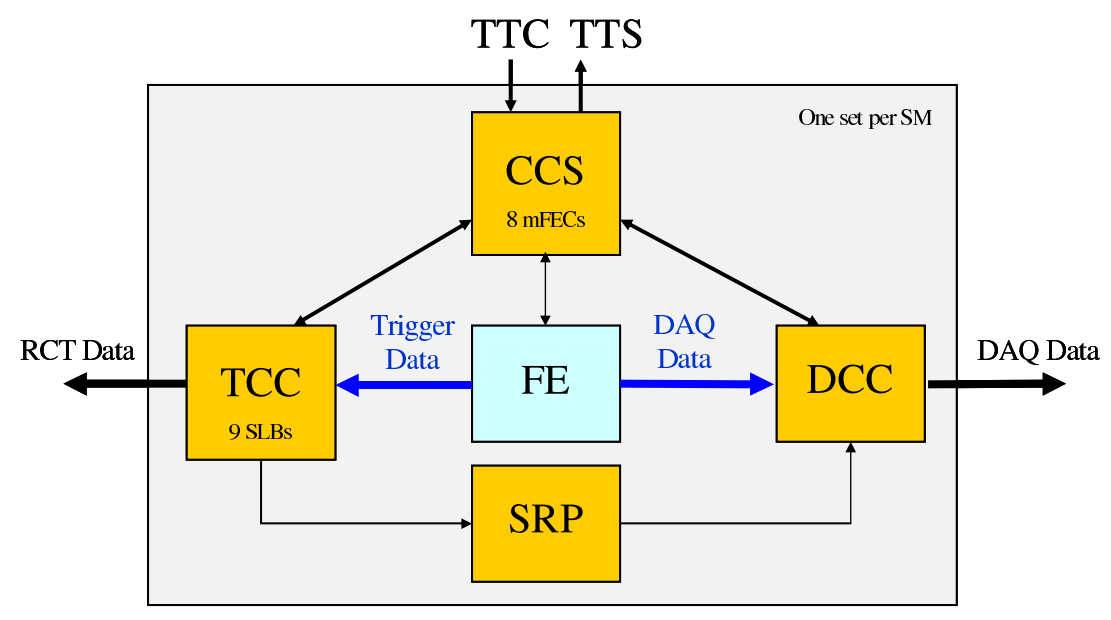

Figure 3.9: Schematic block diagram of the ECAL off-detector electronics system architecture comprising four dedicated boards.

\subsubsection{Clock and control system (CCS)}

The CCS $[28,29]$ performs the configuration and set-up of the on-detector electronics, and distributes the clock, trigger and control signals from the TTC system to the on-detector as well as to the off-detector electronics. Furthermore, it provides interface between the TCC/DCC boards and the Trigger Throttling System (TTS) ${ }^{8}$.

A schematic view of the CCS functionality is depicted in Fig 3.10. First of all, the CCS performs optical-to-electrical conversion of the TTC signals and after their decoding and extraction of the clock and broadcast commands (specified in Tab. 3.1), the signals are routed to eight mFEC cards. Each mFEC contains a transmit FIFO for data to be sent and a receive FIFO for data coming back from the token ring networks through which the signals are distributed to the FE boards. Therefore, one CCS board controls one whole supermodule. Distribution of the TTC signals to the TCC and DCC boards is provided by a short length backplane bus that is also used for reception of the TTS signals from these boards. The TTS signals are collected and controlled by the CCS and then sent to the TTS system.

In addition, the CCS board contains the QPLL chip which reduces the jitter of the system clock and generates clock when the TTC signal is not available. The slow control of the system is provided through the VME backplane bus.

\subsubsection{Trigger concentrator card (TCC)}

The TCC $[25,30]$ collects the trigger primitives transmitted by the optical link at $40 \mathrm{MHz}$ from the trigger primitives generator of the 68 trigger towers (one supermodule) in the barrel or 48 FE boards in the endcaps corresponding to the inner or outer part of a $20^{\circ}$ sector. A schematic block diagram of the endcap TCC is seen in Fig. 3.11 [2].

\footnotetext{
${ }^{8}$ The Trigger-Throttling System (TTS) protects against buffer overflows. It provides fast feedback from any of the sub-detector front-ends to the Global Trigger Processor (GTP) so that the trigger can be throttled before buffers overflow.
} 


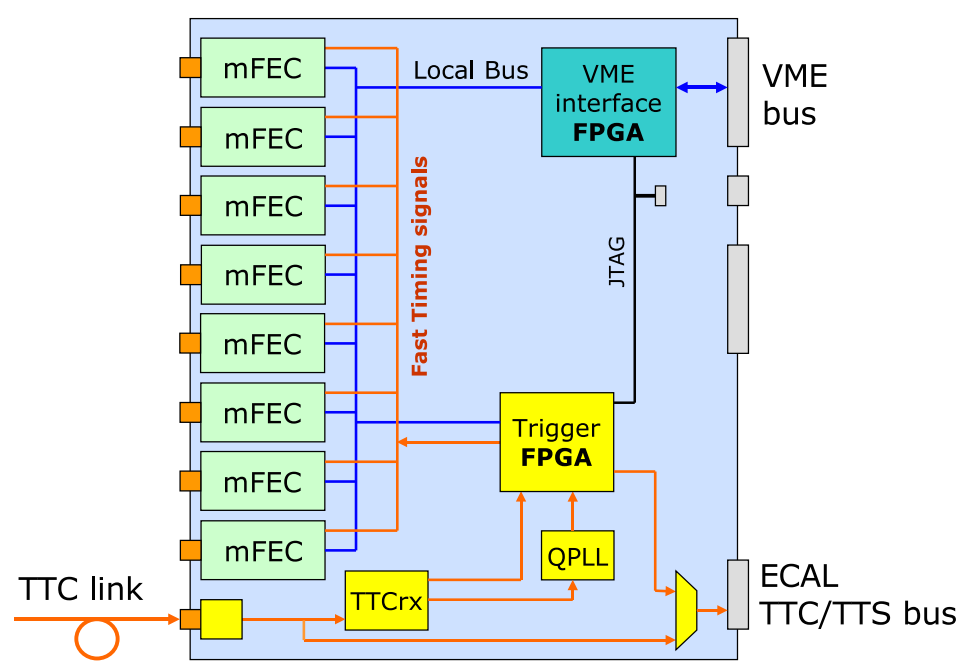

Figure 3.10: Block diagram of the architecture of the Clock and Control System (CCS) board of the ECAL off-detector electronics system.

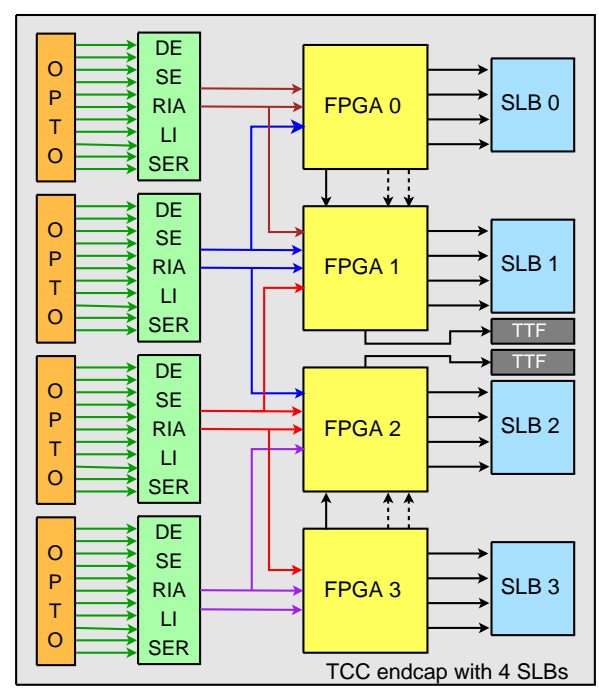

Figure 3.11: Block diagram of the architecture of the endcap Trigger Concentrator Card (TCC) of the ECAL off-detector electronics system. 
After the optical-to-electrical conversion and deserialization (G-Link protocol), data are processed in six or four Xilinx FPGA ASICs in case of the barrel or endcaps, respectively. In the endcap version, the completions of the trigger primitives from the endcap pseudostrips (i.e. the geometrical mapping between the pseudo-strips collected from different supercrystals and the trigger towers and calculation of the FGV bit) is performed. The next steps are common for both the endcap and the barrel. The trigger primitives are finalized by encoding them with a non-linear scale for the total transverse energy $E_{T}^{t o t}$ deposited in the trigger tower. $E_{T}^{\text {tot }}$ is then classified according to adjustable low and high energy thresholds into three categories ${ }^{9}$. The encoded trigger primitives are synchronized with respect to BC0 by the mezzanine Synchronization and Link Board (SLB) [31] and transmitted by a $1.2 \mathrm{Gbit} \mathrm{s}^{-1}$ copper link at $40 \mathrm{MHz}$ to the RCT. When a level-1 trigger accept signal is received, the encoded trigger primitives stored in the TCC are sent together with the tower classification to the DCC. At the same time the classification is also sent to the SRP, which selects the trigger towers that will be readout by the DAQ system.

\subsubsection{Data concentrator card (DCC)}

The DCC $[32,33]$ is in charge of crystal and trigger data collection from up to $68 \mathrm{FE}$ boards and from 1 or 4 TCCs for the barrel and endcap, respectively. The data are received upon level-1 trigger accept signal. The DCC's main functionalities include the optical-to-electrical conversion and deserialisation (8-bit/10-bit protocol) of the input data streams into 16 bit words, the verification of data integrity, the reduction of the data volume, the data event formations and their transmition to the global DAQ.

A schematic block diagram of the DCC architecture is shown in Fig. 3.12 [32]. After the conversion and deserialisation, the data enter to the Receiver Block (RB), which performs the data integrity check, their volume reduction according to the received selective readouts flags from SRP, and data storage in the input iFIFO memories. Then the data are integrated into single packages, called DCC events, by the Event Builder Blocks and stored in the output oFIFO memories. The DDC is designed to store a maximum of 32 events. Now the DCC events are ready for transmission to the global DAQ system by S-link 64 with an average data rate of 200 MByte $^{-1}$ for a level-1 trigger rate of $100 \mathrm{kHz}$. The RB also controls the occupancy of the memories to prevent buffer overflows.

The DCC is also responsible for reading the monitoring data, such as laser pulse, test pulse, pedestals, leakage current and temperature measurements, coming from two monitoring channels (MEMs) and its transmission with spy events to the local DAQ. A VME memory is used for the local DAQ and allows access to physics and monitoring events in spy mode. No data reduction is applied on these events.

\subsubsection{Selective readout processor (SRP)}

In order to accomplish an event size of about $100 \mathrm{kByte}$, which is allocated for the ECAL data in the total CMS event, it is essential to use high level selective readout that reduces an original amount of data by a factor of $\sim 20$ without affecting its physics performance. For this purpose the selective readout processor (SRP) [34] is employed. It applies selective algorithms on level-1

\footnotetext{
${ }^{9}$ Each trigger tower is classified by the TCC as a low $\left(E_{T}^{\text {tot }}<L E T\right)$, medium $\left(L E T<E_{T}^{\text {tot }}<H E T\right)$, or high $\left(E_{T}^{\text {tot }}>H E T\right)$ interest trigger tower for the selective readout. The LET means low energy threshold and HET high energy threshold, and their for the barrel values are about $2.5 \mathrm{GeV}$ and $5 \mathrm{GeV}$, respectively.
} 


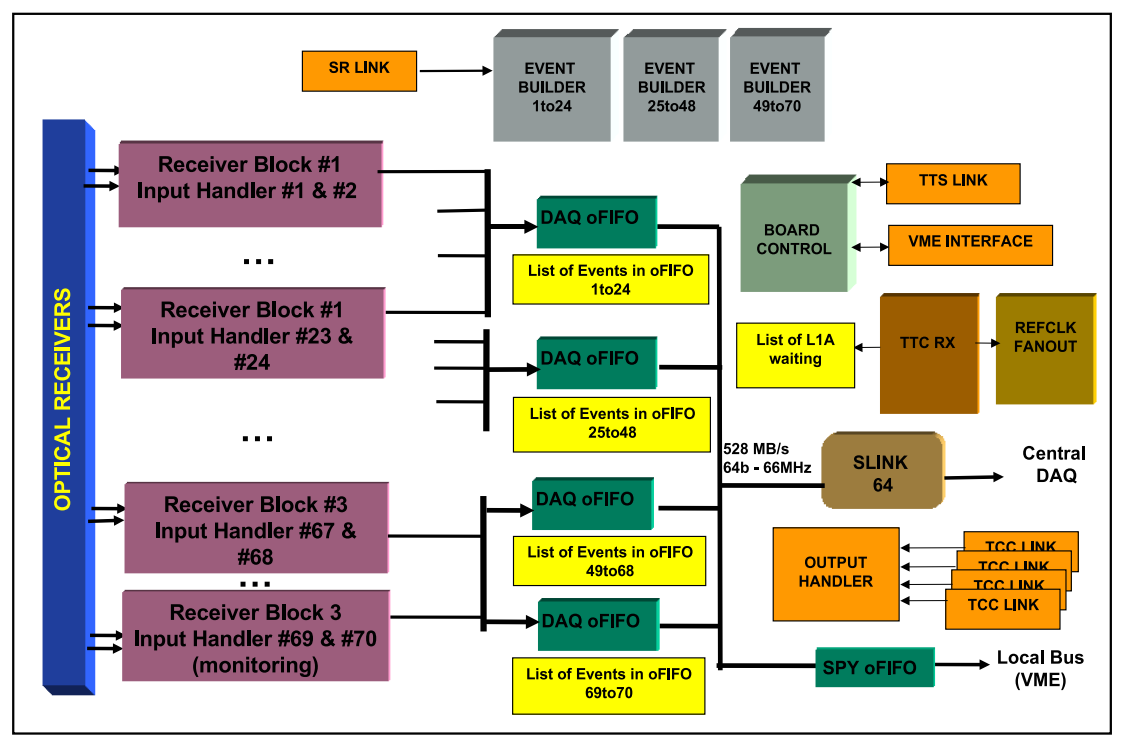

Figure 3.12: Block diagram of the architecture of the Data Concentrator Card (DCC) of the ECAL off-detector electronics system. For the abbreviations see text.

accepted events to determine ECAL areas of interest by evaluating the trigger tower transverse energies $E_{T}^{t o t}$.

The SRP consists of 12 identical Algorithm Boards (ABs) housed in a single 6U VME64x crate and serves the whole ECAL. It receives from the 108 TTCs the classification of trigger towers as described in Chap. 3.4.2 and computes selective readout flags (SRFs) indicating the suppression level that must be applied by the 54 DDCs to the associated readout units. The SRP scans the trigger towers in the whole ECAL in $\phi$ and $\eta$ directions and use the following algorithm to select the appropriate level of suppression: If a trigger tower is flagged as a trigger tower with high interest, then the crystals of this trigger tower and all adjacent trigger towers are read with no zero suppression, hence the DCC reads all the crystals. If a trigger tower belongs to the medium interest, then only crystals of this tower are read without zero suppression. And finally, if a trigger tower is marked as low interest and it is not the neighbour of a high interest tower, then its crystals are read with zero suppression at about $3 \sigma_{\text {noise }}$.

The SRFs must be delivered to the DCC within $6.4 \mu \mathrm{s}$, the maximum time during which the crystal data are stored in DCC input pipelines. Then the DCC uses the SRFs to make on-line data volume reduction [34].

\subsection{Conclusion}

The ECAL electronics, being the most complex system of the calorimeter, have been presented with no respect to the completeness in this chapter. Both, the on-detector and off-detector systems have been described. The new concept of the front-end electronics, for which were decided in 2003, have been successfully adapted and finalized with regard to a very limited time. The very-front-end electronics, which was only briefly outlined in this chapter, will be presented in detail in what follows. 



\section{Bibliography}

[1] H. Rykaczewski et al., Changes to CMS ECAL electronics addendum to the Technical Design Report, ser. Technical Design Report CMS. Geneva: CERN, 2002.

[2] P. Busson, "Overview of the new CMS electromagnetic calorimeter electronics," in $8^{\text {th }}$ Workshop on Electronics for LHC Experiments LECC 2002, Colmar, France, Sep 2002, pp. 324-328.

[3] W. Lustermann et al., "Low voltage supply system for the very front end readout electronics of the CMS electromagnetic calorimeter," in $9^{\text {th }}$ Workshop on Electronics for LHC Experiments LECC 2003, Amsterdam, The Netherlands, Oct 2003, pp. 353-357.

[4] — - "Low voltage supply system for the very front end readout electronics of the CMS electromagnetic calorimeter," in $5^{\text {th }}$ Workshop on Electronics for LHC Experiments LECC 1999, Snowmass, USA, Sep 1999, pp. 397-402.

[5] "ST microelectronics LHC radiation hardened voltage regulator," http://lhc-voltageregulator.web.cern.ch/LHC-Voltage-regulator/default.htm.

[6] J. Blaha et al., "Performance of CMS ECAL very front end electronics," in 12 $2^{\text {th }}$ Workshop on Electronics for LHC Experiments LECC 2006, Valencia, Spain, Sep 2006, pp. 174-178.

[7] — - "Calibration of the very-front-end electronics for the electromagnetic calorimeter of the CMS experiment," Czechoslovak Journal of Physics, vol. 56, no. 8, pp. 879-881, Aug 2006 .

[8] J. P. Walder et al., "Custom integrated front-end circuit for the CMS electromagnetic calorimeter," IEEE Trans. Nucl. Sci., vol. 48, no. LYCEN-2000-110. 6 II, pp. 2375-2379, Oct 2000 .

[9] M. Raymond et al., "The MGPA electromagnetic calorimeter readout chip for CMS," in $9^{\text {th }}$ Workshop on Electronics for LHC Experiments LECC 2003, Amsterdam, The Netherlands, Oct 2003, pp. 83-87.

[10] G. Minderico et al., "A CMOS low power, quad channel, 12 bit, 40MS/s pipelined ADC for applications in particle physics calorimetry," in $9^{\text {th }}$ Workshop on Electronics for LHC Experiments LECC 2003, Amsterdam, The Netherlands, Oct 2003, pp. 88-91.

[11] M. Hansen, Front-End Card version 2.5 (2e) Specification, CERN - The CMS ECAL Front End Electronics Group, Geneva, Jan 2005.

[12] — "The new readout architecture for the CMS ECAL," in $9^{\text {th }}$ Workshop on Electronics for LHC Experiments LECC 2003, Amsterdam, The Netherlands, Oct 2003, pp. 78-82. 
[13] S. Bimbot et al., "Tests of the CMS-ECAL trigger primitive generation," CERN, Geneva, Tech. Rep. CMS-CR-2004-027, Jul 2004.

[14] P. Moreira et al., "A radiation tolerant gigabit serializer for LHC data transmission," in rth Workshop on Electronics for LHC Experiments LECC 2001, Stockholm, Sweden, Sep 2001, pp. 145-149.

[15] M. Hansen, FENIX ASIC version 2.0, HDL description version 1, Specification, CERN The CMS ECAL Front End Electronics Group, Geneva, May 2003.

[16] — - "CMS ECAL FENIX ASIC design methodology," in $10^{\text {th }}$ Workshop on Electronics for LHC Experiments LECC 2004, Boston, USA, Sep 2004, pp. 362-365.

[17] D. J. MacKay, Information Theory, Inference, and Learning Algorithms. Cambridge University Press., 2003.

[18] C. Paillard, C. Ljuslin, and A. Marchioro, "The CCU25a network oriented communication and control unit integrated circuit in a $0.25 \mu \mathrm{m}$ CMOS technology," in $8^{\text {th }}$ Workshop on Electronics for LHC Experiments LECC 2002, Colmar, France, Sep 2002, pp. 174-178.

[19] A. Marchioro, C. Ljuslin, and C. Paillard, CCU25 Communication and Control Unit ASIC for Embedded Slow Control, CERN, Geneva, Jan 2005, cCU25 Specification v2-1, Draf 2.1 .

[20] J. Grahl, "Optical data links for the CMS ECAL," in $11^{\text {th }}$ International Conference On Calorimetry In High Energy Physics - CALOR 2004, Perugia, Italy, Apr 2004, manuscript not submitted to the proceedings.

[21] P. Moreira et al., Gigabit Optical Link Transmitter manual, Version 1.9, CERN, Geneva, Oct 2005, cERN Microelectronics group.

[22] F. Vasey, CMS Tracker Optical Readout Link Specification, Part 2.2: Laser Transmitter, Version 3.7, CERN, Geneva, Oct 2003, CERN EP/CMS, CMS-TK-ES-0004.

[23] A. Dolgopolov and A. Singovski, "Token ring link board - CMS ECAL fronted optical-toelectrical token ring converter," CERN, Geneva, Tech. Rep., April 2005.

[24] F. Vasey and K. Gill, CMS Tracker Optical Control Link Specification, Part 2: Front-end Digital Optohybrid, Version 2.2,, CERN, Geneva, Nov 2003, cERN EP/CMS, CMS-TKES-0019.

[25] R. Benetta et al., "CMS ECAL off-detector electronics," CERN, Geneva, Tech. Rep. CMSCR-2004-022, Jun 2004.

[26] CMS Collaboration, CMS TriDAS projectTechnical Design Report; 1, the trigger systems, ser. Technical Design Report CMS. Geneva: CERN, 2000.

[27] "CERN S-link homepage," http://hsi.web.cern.ch/HSI/s-link/.

[28] K. Kloukinas, "The CMS ECAL readout architecture and the clock and control system," in $11^{\text {th }}$ International Conference On Calorimetry In High Energy Physics - CALOR 2004, Perugia, Italy, Apr 2004. 
[29] K. Kloukinas et al., "FEC-CCS: A common front-end controller card for the CMS detector electronics," in 12 ${ }^{\text {th }}$ Workshop on Electronics for LHC Experiments LECC 2006, Valencia, Spain, Sep 2006, pp. 179-184.

[30] P. Paganini et al., "Tests of the boards generating the ECAL trigger primitive in CMS: From the on-detector electronics to the off-detector electronics systems." in $10^{\text {th }}$ Workshop on Electronics for LHC Experiments LECC 2004, Boston, USA, Sep 2004, pp. 248-252.

[31] N. Almeida et al., "Calorimeter trigger synchronization in the CMS experiment," Nucl. Instrum. Methods Phys. Res., A, vol. 568, pp. 634-641, 2006.

[32] — - "Data concentrator card and test system for the CMS ECAL readout," in $9^{\text {th }}$ Workshop on Electronics for LHC Experiments LECC 2003, Amsterdam, The Netherlands, Oct 2003, pp. 115-118.

[33] R. Alemany et al., "Test results of the data concentrator card of the CMS electromagnetic calorimeter readout system," in $10^{\text {th }}$ Workshop on Electronics for LHC Experiments LECC 2004, Boston, USA, Sep 2004, pp. 217-221.

[34] N. M. Girõ de Almeida et al., "The selective read-out processor for the CMS electromagnetic calorimeter," IEEE Trans. Nucl. Sci., vol. 52, no. 3 pt.2, pp. 772-777, 2005. 



\section{Chapter 4}

\section{Very-Front-End electronics}

\subsection{Introduction}

The very-front-end electronics is an important part of the front-end system integrated on a custom Printed Circuit Board (PCB) named Very-Front-End (VFE) card. Almost 16,000 VFE cards are needed for the whole ECAL, where five cards per a readout unit, a trigger tower, process signals coming from the APDs or VPTs in the barrel or endcap, respectively. A design review of the VFE card and its individual components, their performance characteristics and results of the most relevant tests are given in this chapter.

\subsection{Very-front-end card}

Since physics requirements and photodetectors are different in the barrel and the endcap regions, the VFE card was designed in two types: covering a dynamic range up to $50 \mathrm{pC}$ corresponding to an incident particle energy of $\sim 1.7 \mathrm{TeV}$ for barrel and $16 \mathrm{pC}$ for energy up to $\sim 3.5 \mathrm{TeV}$ for the endcaps. Both types comprise five identical and independent readout channels. Each channel, processing the signal from one crystal, consists of a Multi-Gain Pre-Amplifier (MGPA), a multi channels Analogue-to-Digital Convertor (ADC) AD41240, and two low voltage differential adapters LVDS-RX. The VFE ASICs are identical for both types of the card and the different dynamic ranges are set by external electrical components. A schematic drawing of such a VFE channel is displayed in Fig. 4.1.

The MGPA chip contains a low noise pre-amplifier and three parallel gain stages with nominal gains 1, 6, and 12 that shape and amplify the photodetector signal. The three analogue output signals of the MGPA are then digitalized in parallel by the multi-channel $40 \mathrm{MHz} 12-$ bit ADC AD41240. An ADC internal logic determines whether a gain is saturated and then outputs the data from the highest non-saturated channel. The Low Voltage Differential Signal (LVDS) outputs of the ADC are adapted by two LVDS-RX chips to the single ended inputs of the front-end (FE) board, which thus receives the 12 bit digitalized pulse information and the 2 bit identification of the gain. This configuration allows the digitalization of signal at 40 MSamples $\mathrm{s}^{-1}$ at 16 bit equivalent precision and thus fulfill the ECAL required performance.

The MGPA chip also includes a test pulse unit with an integrated Digital-to-Analogue Converter (DAC) programmed via an $\mathrm{I}^{2} \mathrm{C}$ interface, which allows the channel functionality to be checked by injecting test charges directly into the input of the pre-amplifier. The pedestal value can be set independently for each gain. 


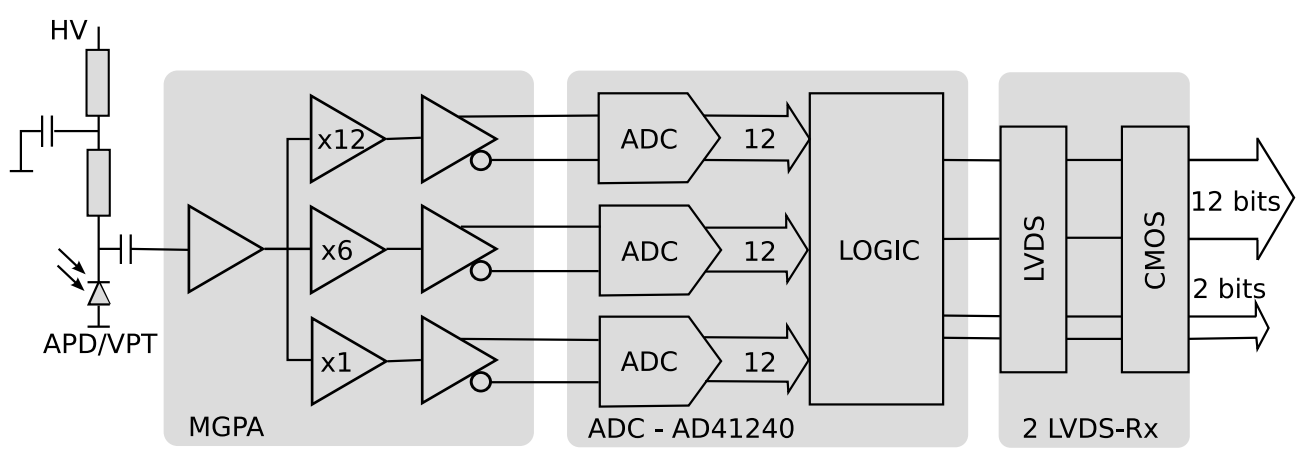

Figure 4.1: Schematic representation of one channel of the Very-Front-End (VFE) architecture comprising the MGPA, 12-bit multi-channel ADC AD41240 and two level adapters LVDS-RX.

In addition, the VFE board also incorporates a Detector Control Unit (DCU) chip for measuring the crystal temperature and, in case of the barrel, also the APD leakage current. All the chips are implemented in $0.25 \mu \mathrm{m}$ IBM CMOS radiation-hard technology [1], which also allows to power all the chips by the same regulated voltages $(2.5 \mathrm{~V})$.

The average power consumption measured for 420 pre-series VFE cards [2] is $\sim 2.3 \mathrm{~A}$ at $2.5 \mathrm{~V}$ corresponding to $1.15 \mathrm{~W} /$ channel. For five channels of a VFE card, $1.2 \mathrm{~A}$ are used by the MGPA and the analog part of the ADC, $0.97 \mathrm{~A}$ by the digital part of the ADC and, $0.12 \mathrm{~A}$ by the LVDS-RX buffers and the DCU.

\subsection{Multi-Gain Pre-Amplifier}

The MGPA chip [3-5], which is designed to be common for the barrel and endcaps regions, meets all the target requirements that are listed in Tab. 4.1 [5]. As is seen in this table, the full-scale signal, noise level and input capacitance parameters have specific values for the barrel and end-cap, which is due to the various full-scale energy requirements, as well as the different detector characteristics of the APDs and VPTs. The three MGPA internal gain stages $(1,6$, and 12) and their ratios are identical for the whole calorimeter and have been chosen according to the targeted ECAL physics performance. On the other hand, the overall gains have been set individually for the barrel and endcap by the external electrical components housed on the VFE PCB. In the case of the barrel, where there are two APDs per crystal, it must be taken into account that the combined APD capacitance, which dominates the overall MGPA input capacitance contrary to the endcap, where interconnect capacitance dominates since the VPT contribution is negligible.

Since the electronics noise increases with the fast shaping time contrary to the pile-up noise which is dominant for slow shaping time, the MGPA CR-RC pulse time constant has been chosen to be $40 \mathrm{~ns}$ as a tradeoff between these two noise contributions. Considering that, the effective output peaking time, which includes the pulse shaping time as well as an average of the $\mathrm{PbWO}_{4}$ crystal scintillation decay time ( $\sim 10 \mathrm{~ns})$, is about $50 \mathrm{~ns}$. The MGPA output signal size is defined to match the ADC input specification. 


\begin{tabular}{|l|c|c|}
\hline \hline Parameter & Barrel & End-cap \\
\hline Full-scale signal & $60 \mathrm{pC}$ & $16 \mathrm{pC}$ \\
Noise level & $10,000 \mathrm{e}, 1.6 \mathrm{fC}$ & $3,500 \mathrm{e}, 0.56 \mathrm{fC}$ \\
Input capacitance & $\sim 200 \mathrm{pF}$ & $\sim 50 \mathrm{pF}$ \\
\cline { 2 - 3 } Output signals to & differential $1.8 \mathrm{~V} \pm 0.45 \mathrm{~V}$ around \\
match ADC & $1.25 \mathrm{~V}$ common mode voltage \\
Gain ranges $( \pm 10 \%$ & \multicolumn{2}{|c|}{$1: 6: 12$} \\
tolerance) & $30 \mu \mathrm{V} \mathrm{fC}^{-1}: 180 \mu \mathrm{V} \mathrm{fC}^{-1}: 360 \mu \mathrm{V} \mathrm{fC}{ }^{-1}$ \\
Integral nonlinearity & $< \pm 0.1 \%$ full-scale (each range) \\
Pulse shaping & \multicolumn{2}{|c}{40 ns CR-RC } \\
Pulse shape matching & $< \pm 1 \%$ (within and across ranges) \\
\hline \hline
\end{tabular}

Table 4.1: Multi gain pre-amplifier (MGPA) specification [5].
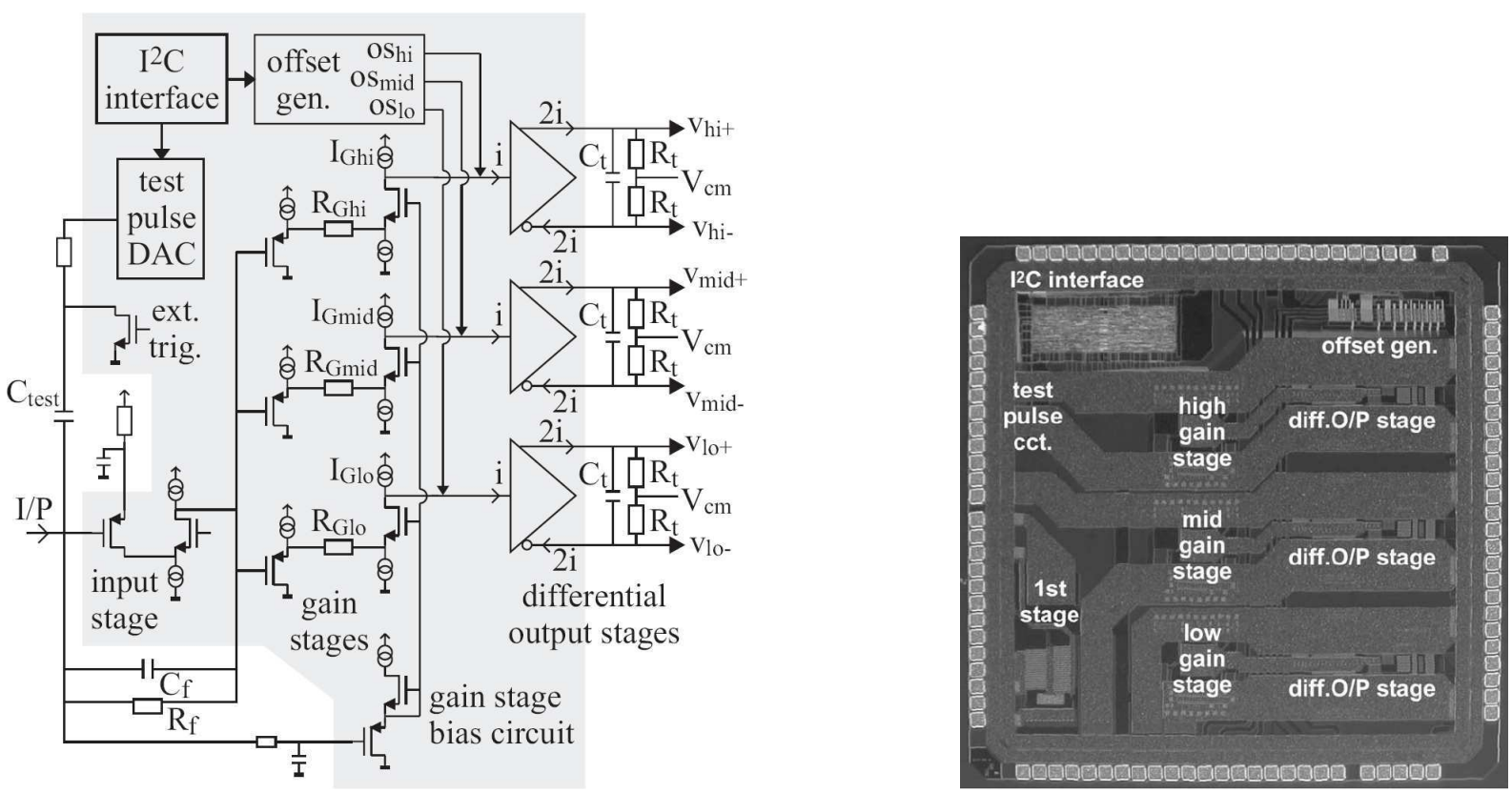

Figure 4.2: Left: Schematic drawing of the MGPA architecture. Components inside the shaded area are on-chip, those outside are external. Right: Photograph of the MGPA die with internal circuitry locations indicated [4]. 


\subsubsection{Electronic architecture}

A schematic drawing of the MGPA chip circuity is displayed in Fig. 4.2 left, where all the internal components are inside the shaded area. Description of the MGPA electronic architecture can be found elsewhere $[4,5]$, here only a short architecture overview is given. The APD or VPT input signals enter the first stage which is a folded-cascade charge sensitive pre-amplifier with an external feedback capacitor $C_{f}$ and resistor $R_{f}$. These components are chosen to match the full-scale signals and their values are $33 \mathrm{pF}, 8.2 \mathrm{pF}$, and $1.2 \mathrm{k} \Omega, 4.7 \mathrm{k} \Omega$ for the barrel and endcap, respectively. The small values of the $C_{f}$ capacitors reduce the noise contributions from subsequent stages.

The output of the first stage is then buffered to the three gain channels, where the internal resistor of each gain channel $\left(R_{G h i}=14 \Omega, R_{G m i d}=34 \Omega\right.$, and $\left.R_{G l o}=240 \Omega\right)$ sets and feeds following common gain stage and provides current to the differential output stage. The final choice of how to distribute the gains between the differential and gain stages was designed as a compromise between performance and power in order to keep low noise and good linearity.

The three differential output stages are for all gain channels identical and created as singleended input to differential output current circuits with output stage terminations resistors $R_{t}=$ $200 \Omega$ providing current-to-voltage conversion and are terminated to a common mode voltage $V_{c m}$ of $1.25 \mathrm{~V}$. To provide $40 \mathrm{~ns} \mathrm{RC}$ integrating time constant of the pulse shaping, the terminations capacitors $C_{t}$ are chosen to be $100 \mathrm{pF}$. The main advantage of the low pass filtering at the end of the signal processing chain is that $C_{t}$ has influence on all the noise sources within the MGPA chip.

An $\mathrm{I}^{2} \mathrm{C}$ (Inter-Integrated Circuit) $)^{1}$ interface is used to program the individual pedestal levels at the outputs by setting the magnitudes of offset currents applied at the differential stage inputs. This is needed because without it the differential output stages will naturally bias up at the midpoint of their dynamic ranges where the magnitudes of the individual output currents are zero. The offset currents for each channel are generated on-chip via the offset generator block, which uses weighted current mirrors and switches to generate an output current according to the value held in the 8-bit $\mathrm{I}^{2} \mathrm{C}$ register. Thus the generator provides the offset currents in range from 0 to $\mathrm{I}_{\max }$ in a 256 -step linear scale.

Fig. 4.2 right shows a photograph of the MGPA die $\left(4 \times 4 \mathrm{~mm}^{2}\right)$ with indication of the main circuit blocks described above. During the design phase, special stress was put on the physical separation of the gain channels to avoid cross-talk when the highest gain range saturates.

\subsubsection{Test pulse unit}

The test pulse unit is integrated in the MGPA chip and allows to inject a test charge into the MGPA input and thus performs a simple functionality test of the chip itself or to verify the complete electronic chain of the ECAL. A test charge is determined by a simple internal DAC circuit using eight serial resistors with switches controlled by $\mathrm{I}^{2} \mathrm{C}$ allowing to select different $\mathrm{dc}$ levels, which are applied on an external charge injection capacitor $\mathrm{C}_{\text {test }}$ (see Fig. 4.2 left) and which is connected to the MGPA first stage input. A test pulse is produced by discharging the capacitor $\mathrm{C}_{\text {test }}$ to the ground at the reception of an external trigger.

\footnotetext{
${ }^{1} \mathrm{I}^{2} \mathrm{C}$ is a serial multi-master bus invented by Philips Semiconductors using bi-directional 2-wire bus for efficient inter-IC control.
} 


\subsubsection{Performance characteristics}

The MGPA chip draws approximately $240 \mathrm{~mA}$ from a single 2.5 Volt rail. The input gain and differential output stages consume approximately 150, 300 and, $150 \mathrm{~mW}$ respectively. The summary of the basic measured characteristics of the final version of the MGPA chip is mentioned below.

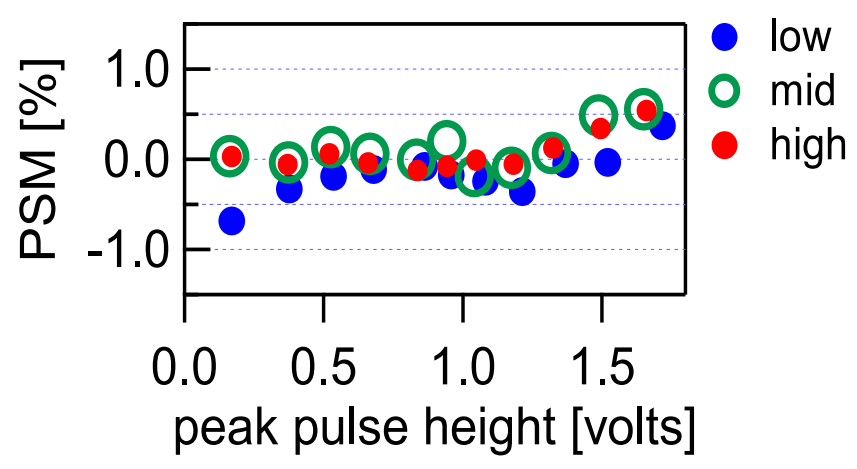

Figure 4.3: MGPA pulse shape matching across and between ranges [4].

\section{Pulse shape}

Pulse shapes were measured for all three gains over the full dynamic range from 0 up to $60 \mathrm{pC}$ for 30 signals not linearly spaced steps with differential signal outputs between 0 and $3 \mathrm{~V}$. It was measured that the high and middle range saturates while the low gain channel remains always in linear range without any visible signs of distortion when the higher ranges are saturated. The average gain ratio measured over 10 chips fulfill well the gain ratio stated in the specification (see Tab. 4.2).

The Pulse Shape Matching (PSM) is given by

$$
P S M[\%]=\frac{P S M F-A P S M F}{A P S M F} \times 100,
$$

where $P S M F$ is the pulse matching factor defined as the ratio of the voltage sample $25 \mathrm{~ns}$ before the peak $\left(V_{\text {peak }}-25\right)$ to the peak voltage sample $V_{\text {peak }}$, and $A P S M F$ is the average over all pulse shapes for all three gain ranges. PSM across and between gain ranges is shown in Fig. 4.3 [5], and agrees well with the $\pm 1 \%$ specification value.

\section{Non-linearity}

Non-linearity (NL) for each value of the input signal amplitude $A$ can be given by

$$
N L[\% \text { fullscale }]=\frac{P H(A)-f i t(A)}{A_{\text {fullscale }}},
$$

where $\operatorname{PH}(A)$ is the measured pulse height of the pulse shape acquired for input signal amplitude $A, f i t(A)$ is the corresponding value from the linear fit to the peak height data set, and $A_{\text {fullscale }}$ 


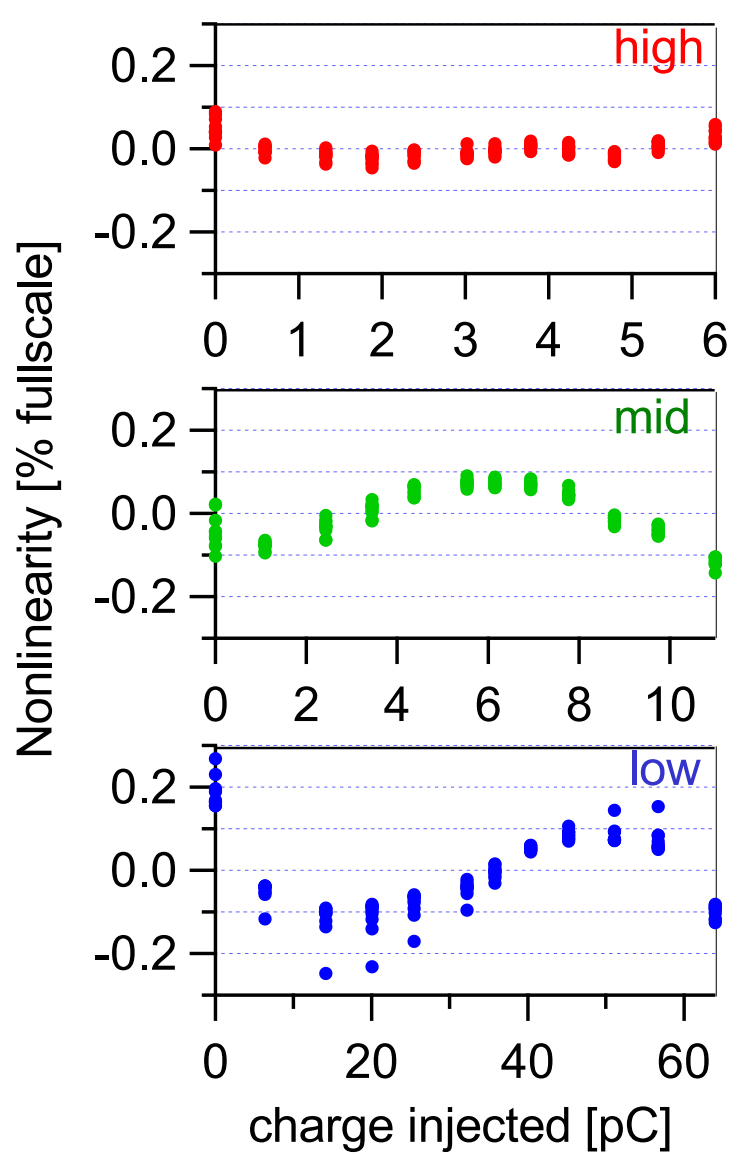

Figure 4.4: MGPA non-linearity measured over the full dynamic range for all three gains [4].

is the signal input amplitude that would generate a full-scale output. The non linearity was measured over the full scale for 10 MGPA chips and is shown for all three gains in Fig. 4.4 [4], where it is clearly seen that for high and middle gain range it is within and, for low range, very close to the $\pm 0.1 \%$ specification. The non linearity stays within the specification also for different values of gain stage bias currents and therefore is not too sensitive to the operating point of the circuit. The non linearity for different values of the bias current is shown for the highest gain in Fig. 4.5 [5].

\section{Noise}

Electronic noise measured for all three gains is listed in Tab. 4.2, which shows that the noise for high and middle gains are within the specifications (see Tab.4.1) contrary to the noise for low gain, which is almost three times higher. It was found that for high and middle gains the input stage noise dominates due to the main contribution from the feedback resistor $R_{f}$ with only small dependence on the input capacitance. Since the gain resistors for both gains are relatively small $\left(R_{G h i}=14 \Omega, R_{G m i d}=34 \Omega\right)$ compared to the low gain resistor $\left(R_{G l o}=240 \Omega\right)$, the overall noise still remains within the specification. Due to the high value of $R_{G l o}$ in case of 


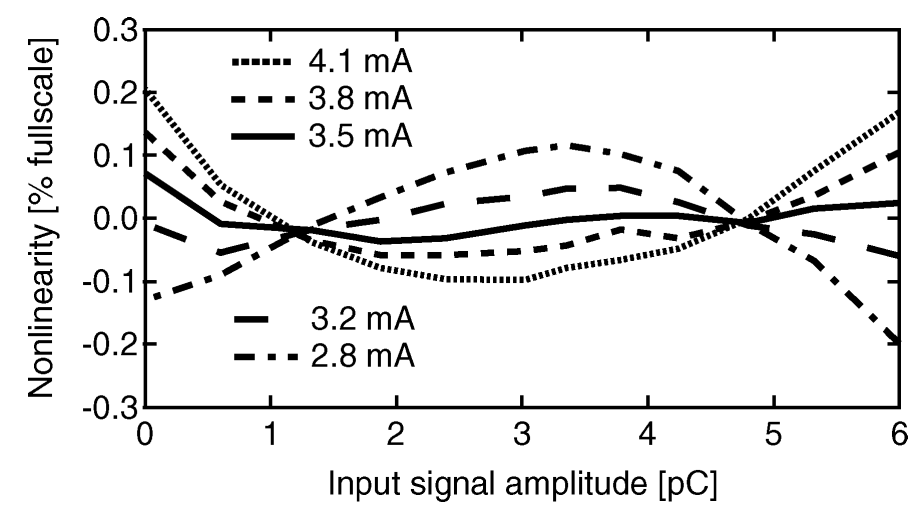

Figure 4.5: MGPA non-linearity measured for different values of gain-stage bias currents for the high gain channel [5]. Similar results were found also for the low and middle gain.

the low gain the gain stage noise is much more significant and becomes the main contribution to the total noise.

The contributions from the gain resistors and common gain stages cannot be reduced due to the limitation of the gain of the input stage to accommodate the large full-scale signals. On the other hand, the higher noise for low gain range, which is used for high energy signals, can be acceptable because its contribution to the overall ECAL energy resolution is negligible.

\begin{tabular}{|l|c|c|}
\hline \hline Gain & Barrel & End-cap \\
\hline high & $8390 \pm 250$ & $3263 \pm 66$ \\
mid & $8850 \pm 265$ & $3496 \pm 122$ \\
low & $27340 \pm 3280$ & $8230 \pm 905$ \\
\hline \hline
\end{tabular}

Table 4.2: MGPA noise expressed as ENC (Equivalent Noise Charge) in electrons measured for all three gains with input capacitances $C_{i n}=200 \mathrm{pF}$ and $C_{i n}=50 \mathrm{pF}$ for the barrel and endcap, respectively [5].

\section{Radiation tolerance}

To confirm radiation hardness, one chip was irradiated with $10 \mathrm{keV}$ X-rays to a dose of $50 \mathrm{kGy}$ $( \pm 10 \%)$ at a dose-rate of $\sim 10 \mathrm{kGy} /$ hour, which is twice the worst case dose expected in CMS after 10 years of LHC operation. The only measurable difference in performance after irradiation was a $3 \%$ reduction in gain for all three channels. No significant degradation of the MGPA performance was observed [5].

\subsubsection{Production procedure}

The production test program of the MGPA [6-8] chips was carried out in collaboration between IPN Lyon and INFN Turin. The preserie sample of the final version of the chips passed an 
initial characterization in IPNL to set out the test production procedure with respect to define appropriate acceptance ranges for specification characteristic (see Tab. 4.1). The test program of 95.000 chips was then performed by $\mathrm{C}^{2} \mathrm{I}^{2}$ using an automatic testing machine with a production rate of 5.000 chips per month. A final production yield of $90 \%$ has been reached.

\subsection{Analogue-to-Digital Converter}

High requirements put on the ECAL read-out electronics led to develop a new Analogue-toDigital Converter (ADC) AD41240 which has a high resolution and sampling speed, low power consumption and an ability to effectively extend the dynamic range of the input signal from the MGPA by the integrated selection logic. In addition, the ADC has to be sufficiently radiation hard. The $\mathrm{AD}_{41240^{3}}[9,10]$ is a 12-bit, four-channel ${ }^{4}$ pipelined ADC capable of running at $40 \mathrm{MHz}$ (an input sampling rate of $40 \mathrm{Ms} \mathrm{s}^{-1}$ and an input signal bandwidth of $5 \mathrm{MHz}$ ) with a power consumption per channel of $150 \mathrm{~mW}$ at $2.5 \mathrm{~V}$.
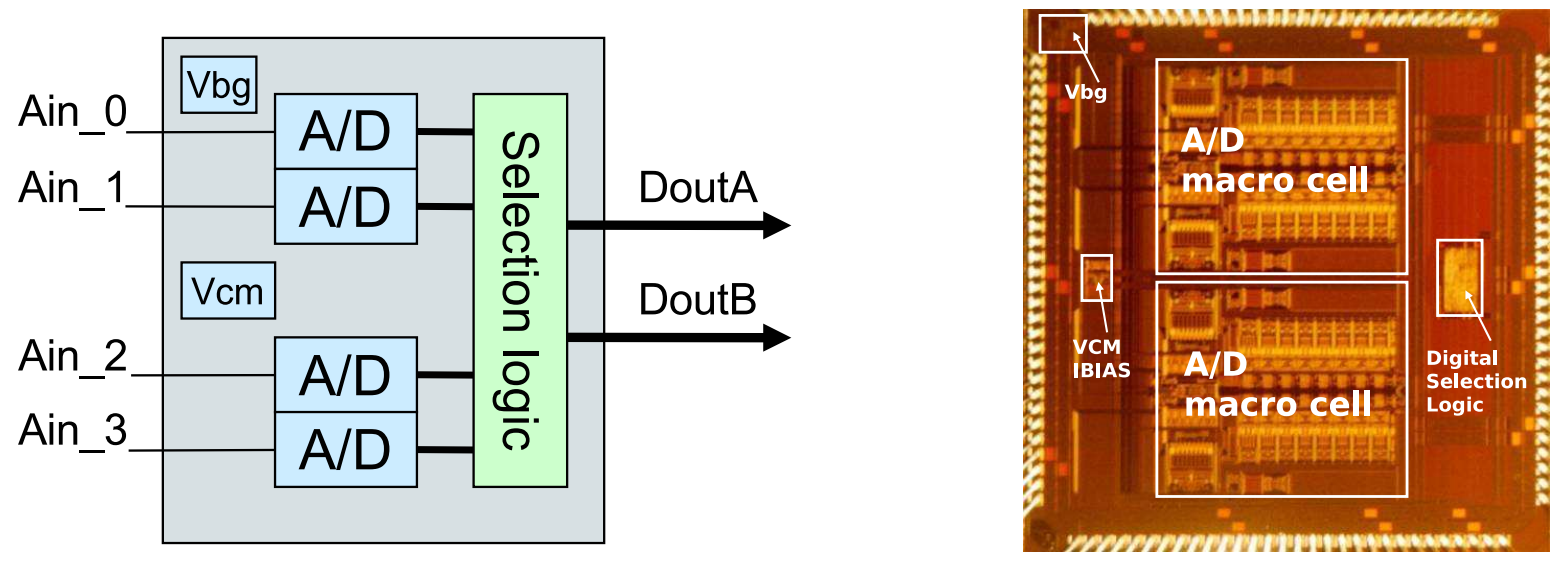

Figure 4.6: Left: Schematic block diagram of the AD41240 showing two ADC macro cells and the range selection logic. $\mathrm{V}_{b g}$ and $\mathrm{V}_{c m}$ blocks represent the bandgap reference generator and the common mode voltage generator, respectively. Right: Microphotograph of the chip with highlighted main functional blocks [10].

\subsubsection{Electronic architecture}

The AD41240 is a quad channel ADC implemented using two dual 12 bit ADC macro cells as is shown in the schematic block diagram in Fig. 4.6. This arrangement allows the best sharing of all the common circuitry parts between channels. Both ADC macro cells thus can use biasing from a common mode voltage generator and current biasing circuit $\left(\mathrm{V}_{\mathrm{cm}}\right)$ and from a bandgap voltage reference generator $\left(\mathrm{V}_{b g}\right)$.

\footnotetext{
${ }^{2}$ C4I (Centre de Compétences en Conception de Circuits Intégrés), Archamps, France

${ }^{3}$ Joint development of CERN and ChipIdea Microelectronica S.A., Lisbon, Portugal

${ }^{4}$ Since the MGPA has only three gain stages, the AD41240 works in a operating mode when only free ADC channels are actively used.
} 
The converter macro cell uses a 10 stages pipeline with multiple resolution and converts in 5 clock cycles. The input signal is directly sampled and quantized in a 2.5 bit front-end stage, which consists of a 2.5 bit MDAC and 2.5 bit flash quantizer, both are fully-differential, and well optimized to match time constants and thus minimize phase shift errors. Then the corresponding residue is propagated in a backend $\mathrm{ADC}$, which is a 10 bit conventional 1.5 bit stage pipeline $\mathrm{ADC}$ as is shown in a schematic block diagram in Fig. 4.7. The resolution per stage in final architecture was carefully chosen as a tradeoff among performance, modularity and complexity of its implementation. It is well-known that large resolution in the front-end stage has better static linearity, distortion and level of generated spurs. On the other hand, the architecture is less modular and more complex due to the amplifiers that are needed for the residue amplification. In case of lower resolutions in the front-end stage, the implementation is simpler and more modular, also it has a faster time response, but it affects achievable performance. A detailed description of the macro cell design can be found in [9].

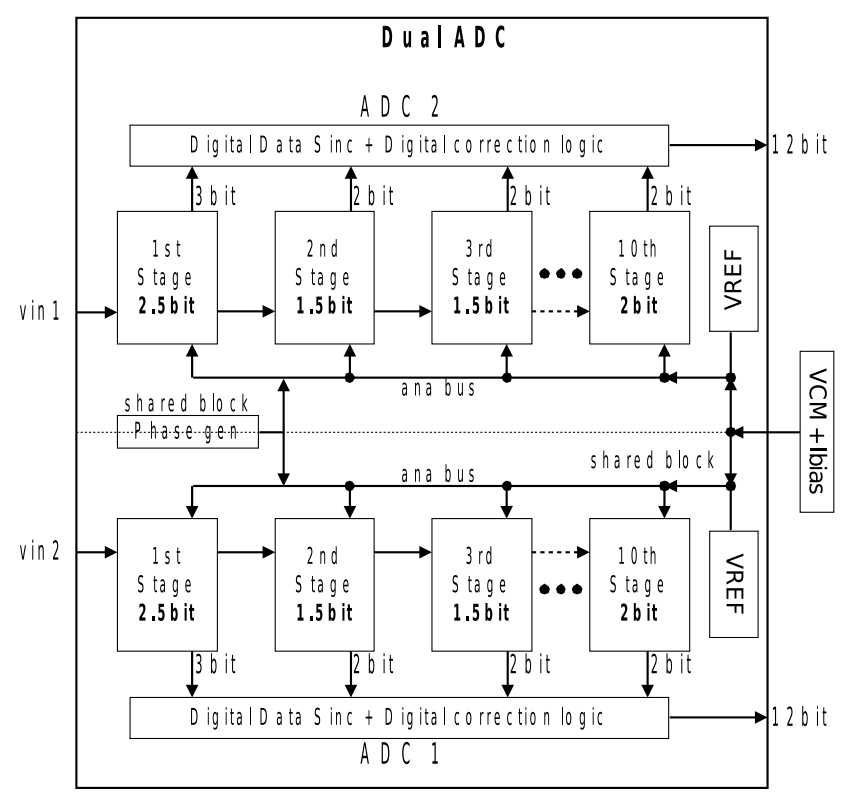

Figure 4.7: Schematic of the ADC macro cell - dual 12 bit data converter [9].

From the last correction stage of the ADC the four digitalized outputs of the ADC macro cell are then routed to a digital selection logic block which performs the choice of the correct input range and thus allows to effectively extend the overall dynamic range of the signal from MGPA. The logic is employed since the multiple gain ranges are used to spam the overall dynamic range of almost 16 bits, digitalizing and transmitting the signal only for the highest unsaturated gain stage. This technical solution does not require a very high precision ADC, and in fact, a 12 bit precision is fully sufficient for this application. The selection logic is implemented as a digital logic which allows more sophisticated channel selection than the analog one. The range selections are performed by digital comparators (one per channel) which decide if the output of the ADC is close to the top of the corresponding range, and in this case it informs an output multiplexer to select the next non-saturated channel. This selection process lasts a very short time and does not add any significant latency to the overall conversion time. Finally, the digitalized signal is 
brought out of the chip by output buses using the LVDS signaling. The LVDS, which was chosen to suppress the noise generated by the digital I/O signals, is also used for reference clock input.

\subsubsection{Operation modes}

The internal selection logic also implements a set of operating modes which permits to configure chip behaviors in terms of defining the width, operation protocol, and speed of the output bus. The modes are listed in Tab. 4.3.

\begin{tabular}{|c|l|l|l|}
\hline \hline Mode & Description & Speed [MHz] & Width [bits] \\
\hline 0 & Quad ADC & 80 DDR & $2 \times 12$ \\
1 & Ecal Direct & 40 & $1 \times 14$ \\
2 & Ecal with Hysteresis & 40 & $1 \times 14$ \\
3 & Ecal Direct & 80 DDR & $1 \times 7$ \\
4 & Ecal with Hysteresis & 80 DDR & $1 \times 7$ \\
5 & Transparent (0-1) & 40 & $2 \times 12$ \\
6 & Transparent (2-3) & 40 & $2 \times 12$ \\
7 & Invalid & & \\
\hline \hline
\end{tabular}

Table 4.3: Valid output bus configurations of the AD41240. The DDR indicated "Double Data Rate" regime, when output clock runs at $40 \mathrm{MHz}$, but data are available on both edges of the clock.

In mode 0 the AD41240 works as a quad ADC with four independent Analogue-to-Digital Converters sharing two multiplexed 12 bit output data buses operating in Double Data Rate (DDR) mode at $80 \mathrm{MHz}$. In fact, the output clock runs at $40 \mathrm{MHz}$, but data are available on both edges of the clock. The AD41240 operating in this quad mode is used in the front-end read-out electronics system of the CMS Preshower detectors [11].

In modes 1 and 2, the AD412140 behaves as a single ADC with a 14 bit width and an output rate of $40 \mathrm{MHz}$, which brings to the output bus only the data from the first ADC channel that is not saturated along with two range selection bits. The ADC channel is considered as a saturated channel if the 8 most significant bits are all equal to "1". The least significant channel is channel 0 and the most significant channel is channel 3. The difference between these two modes is that mode 2 also introduces a digital hysteresis feature. This allows to keep digitalizing a given pulse for 5 clocks on the selected scale for its peak even when the signal decreases. After 5 clock cycles, the ADC restarts selecting its output as usual, i.e. chooses the first unsaturated channel. The main advantage of the hysteresis mode is that it allows to perform the sampling of a pulse within one ADC channel and thus avoid inter-channel calibration problems for signals spanning different scales. Mode 2 is currently used in the ECAL very front end system.

Modes 3 and 4 offer the same functionality as modes 1 and 2, but the output bus is configured to run only on 7 bits at the DDR mode $(80 \mathrm{MHz}$ ). The last two modes (5 and 6) permit to route the ADC buses via selection logic to the output of the chip and thus are used mainly for debugging purposes.

Another important feature of the AD41240 is that the unused channel/channels can be put into the standby operation mode which considerably reduces the power consumption. For instance, if two channels are actively used, the power is reduced almost a factor of two. This 
feature can also be very useful for measurements when only one selected MGPA channels needs to be used, such as the measurements of the MGPA gain ratio, where the the same input signal is measured for each gain separately. This kind of measurement will be here reported as the measurement in forced mode.

\subsubsection{Performance characteristics}

The AD41240 performance parameters were obtained by performing Digital Signal Processing (DSP) techniques on the collected digitalized data. The extracted parameters can be divided into two basic groups: static and dynamic.

\section{Static parameters}

Static parameters quantify errors affecting ADC accuracy when it is converting static (dc) signals. Differential and integral non linearity $[12,13]$ are the two most important static characteristics which represent non linearity errors of an ADC. They can be both delivered by applying the Code Density Test [14], e.g. from the deviation histogram from the probability density function of a sine wave signal converted by an ideal ADC. Generally, the higher values of DNL limit the ADC's performance in terms of signal-to-noise ratio (SNR) and spurious-free dynamic range (SFDR).

Differential Non Linearity (DNL) represents the variation of the any digital code from an ideal 1 LSB (Least Significant Bit) step and is defined as

$$
D N L(D)=\frac{V_{D+1}-V_{D}}{V_{L S B \_I D E A L}}-1, \text { where } \quad 0<D<2^{N}-2 .
$$

$V_{D}$ is the physical value corresponding to the digital output code $D, N$ is the ADC resolution, and $V_{L S B_{-} I D E A L}$ is the ideal spacing for two adjacent digital codes and is equal to $1 \mathrm{LSB}^{5}$. The DNL is specified after the static gain error (deviation from the ideal slope of the transfer function $^{6}$ ) is removed and usually is expressed in fractional bits or as a percentage of the full scale. The differential non linearity error is zero, if the physical width is exactly equal to 1 LSB. If DNL is -1 there is a possibility of one or more missing codes, i.e. one or more of the possible $2^{N}$ binary codes are never output. If DNL exceeds $1 \mathrm{LSB}$, there is a possibility that the converter can be non monolithic. In this case, the magnitude of the output gets smaller for an increase in the magnitude of the input.

Magnitude of the DNL versus digital output code represent the DNL spectrum which is shown for one ADC channel of the AD41240 in Fig.4.8 top [10]. The spectrum was obtained at $40 \mathrm{MHz}$ with an input frequency of $2.5 \mathrm{MHz}$. The maximal value of DNL is $\pm 0.35 \mathrm{LSB}$, which guarantees a monolithic transfer function with no missing codes.

Integral non linearity is the maximum deviation (in LSB or percent of full-scale range) of the transfer function from a straight line between two points along the input-output transfer curve. It is presented as the integral of the DNL errors, so that is why good INL also guarantees good DNL. INL, as a static parameter, relates to total harmonic distortion (THD) which is an

\footnotetext{
${ }^{5} 1 L S B=V_{F S R} /\left(2^{N}-1\right)$, where $V_{F S R}$ is the full-scale range in Volts and $N$ is the resolution of the ADC

${ }^{6}$ The transfer function for an ideal ADC is a staircase in which each tread represents a particular digital output code and each riser represents a transition between adjacent codes
} 

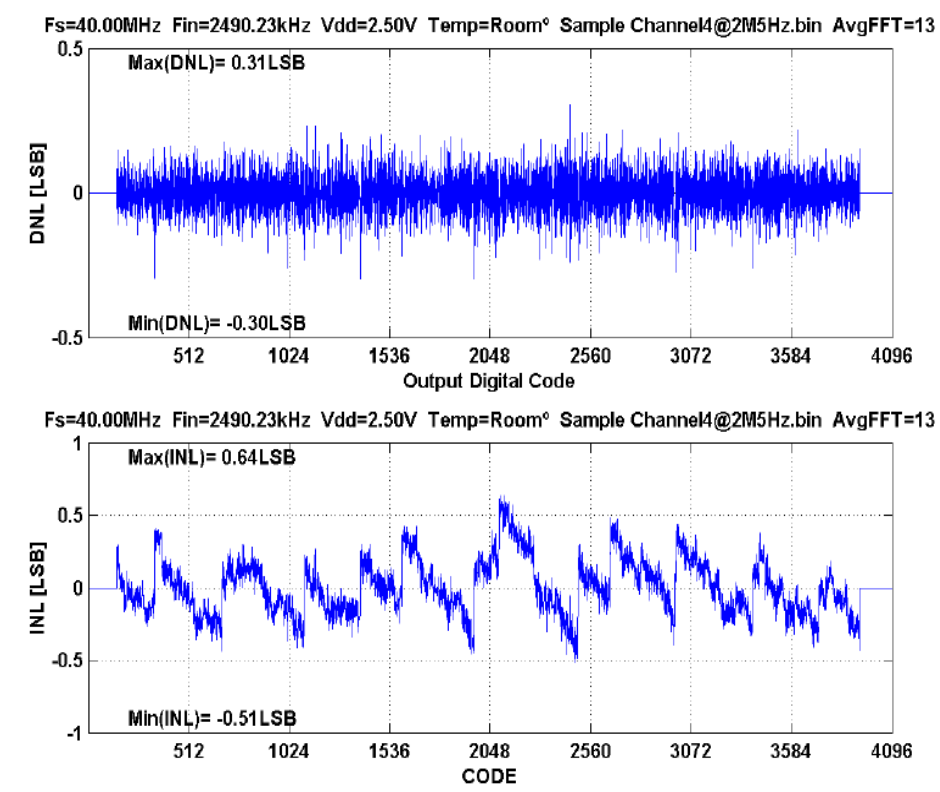

Figure 4.8: Typical spectrum of the differential non linearity (top) and integral non linearity (below) for the input frequency of $2.5 \mathrm{MHz}$ for one ADC channel [10].

important dynamic specification and tends to be worse as the value of INL increases. If both static offset and gain errors are removed, INL can be described as:

$$
I N L(D)=\frac{V_{D}-V_{Z E R O}}{V_{L S B \_I D E A L}}-D, \text { where } \quad 0<D<2^{N}-1 .
$$

$V_{D}$ is the analog value represented by the digital output code $\mathrm{D}, N$ is the resolution of the ADC, $V_{Z E R O}$ is the minimum analog input corresponding to an all-zero output code, and $V_{L S B_{-} I D E A L}$ is the ideal spacing for the adjacent output codes. Typical spectrum at $40 \mathrm{MHz}$ for an input frequency of $2.5 \mathrm{MHz}$ of one AD41240 channel is displayed in Fig.4.8 below [10] which shows a maximal value of INL \pm 0.65 LSB.

\section{Dynamic parameters}

Dynamic parameters of the converter help to evaluate ADC performances when AC signals are considered. The key specifications in this category with their definitions are given below according to ref. [15].

Signal-to-Noise Ratio (SNR) - SNR is the ratio of the RMS signal amplitude for the analog input signal $A_{S I G N A L}$ and the RMS sum of all noise sources $A_{N O I S E}$, excluding harmonics and dc. The SNR is usually expressed in $d B$ and for a sinusoidal input signal can be described as:

$$
S N R=20 \log \frac{A_{S I G N A L}[R M S]}{A_{N O I S E}[R M S]} \approx 6.02 N+1.76,
$$

where $N$ is the resolution of the ADC. 
Total Harmonic Distortion (THD) - THD is the ratio of the RMS sum of all RMS amplitudes $A_{H D(n)[R M S}$ of the harmonics to the RMS value of the fundamental amplitude $A\left[f_{I N}\right]_{R M S}$ and expressed in $d B$ can be written as:

$$
T H D=20 \log \frac{\sqrt{\left.\sum_{n=2}^{N} A_{H D(n)[R M S}^{2}\right]}}{A\left[f_{I N}\right]_{R M S}}
$$

Signal-to-Noise and Distortion Ratio (SINAD) - SINAD is the ratio of the RMS signal amplitude $A_{S I G N A L}[R M S]$ and $A_{N O I S E+H D}[R M S]$ representing the RMS sum of all spectral components below the Nyquist frequency, including harmonics but excluding dc. In $d B$ it is expressed as:

$$
S I N A D=20 \log \frac{A_{S I G N A L}[R M S]}{A_{N O I S E+H D}[R M S]}
$$

Effective Number of Bits (ENOB) - ENOB provides a measure on an ADC's performance. Expressed in bits, it is frequently given by:

$$
E N O B=\frac{S I N A D-1.76}{6.02}
$$

Spurious-Free Dynamic Range (SFDR) - SFDR is the ratio of the RMS value of the fundamental amplitude $A\left[f_{I N}\right]_{R M S}$ to the RMS value of the next-largest spurious component $A\left[f_{I N}\right]_{R M S}$ observed over the entire Nyquist band. In decibels is expressed as:

$$
S F D R=20 \log \frac{A\left[f_{I N}\right]_{R M S}}{A_{S P U R_{M} A X}[R M S]}
$$

The parameters defined above are commonly used for the evaluation of the ADC performance and accuracy at a specific input signal frequency and sampling rate. A Fast Fourier Transform (FFT) based analysis was performed and the dynamic parameters were extracted from the power spectrum plot of the digitalized captured data. The sampling frequency is $40 \mathrm{MHz}$ and several values of input frequency $(1,2.5$, and $10 \mathrm{MHz})$ were applied in these tests. Figure 4.9 shows an example of a FFT power spectrum for one channel of the AD41240 $\left(\mathrm{F}_{s}=40 \mathrm{MHz}, \mathrm{F}_{\text {in }}=\right.$ $2.5 \mathrm{MHz}$ ) together with calculated values of the main dynamic parameters. The fundamental amplitude (indicated as number 1) and its 6 harmonics (numbers 2 - 7) are also clearly seen.

All the most significant parameters obtained with the code density test and FFT spectral analysis for various signal input frequencies are summarized in Tab. 4.4 [10]. One of the most important parameters, which helps to quantify dynamic performance of the ADC is the effective number of bit ENOB already introduced earlier. Its value for the 12 bit AD41240 is 11.1 bit for a sampling and input frequency of $\mathrm{F}_{s}=40 \mathrm{MHz}$ and $\mathrm{F}_{\text {in }}=2.5 \mathrm{MHz}$. Therefore, one can conclude that due to the converter's noise and distortion performance, 1 bit of information is lost, which means that a 12 bit ADC can provide only $0.05 \%$ accuracy at the best.

\subsubsection{Radiation tolerance}

Radiation resistivity was tested on 5 AD41240 samples under bias for doses varying between 0.5 and $100 \mathrm{kGy}$ at a dose rate of $20 \mathrm{kGy} \cdot \mathrm{h}^{-1}$ using CERN microelectronics X-ray source. After an irradiation test, all the samples were found to be functional, but with little impact on the ADC performance parameters. Power consumptions did not increase significantly up to $100 \mathrm{kGy}$ 


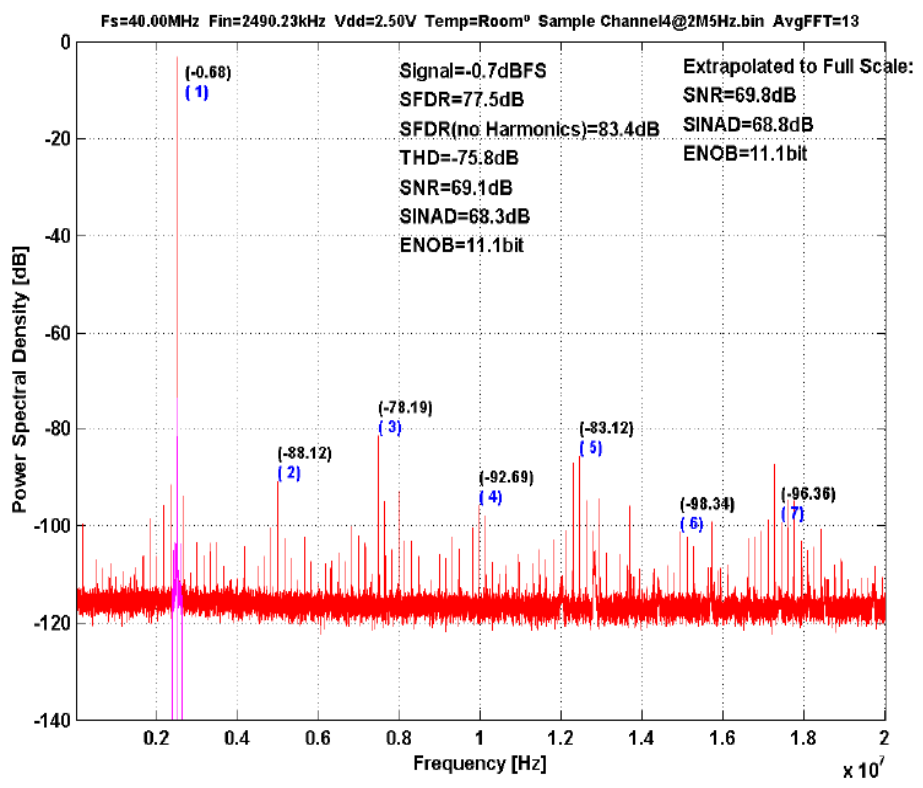

Figure 4.9: Power spectral density versus frequency for the input frequency of $2.5 \mathrm{MHz}$ for one ADC channel [10].

\begin{tabular}{|l|c|c|c|l|}
\hline \hline Parameter & $\mathbf{F}_{i n}=\mathbf{1 ~ \mathbf { ~ M H z }}$ & $\mathbf{F}_{\text {in }}=\mathbf{2 . 5} \mathbf{~ M H z}$ & $\mathbf{F}_{\text {in }}=\mathbf{1 0 ~ \mathbf { M H z }}$ & Units \\
\hline INL & \pm 0.66 & \pm 0.65 & \pm 0.77 & LSB \\
DNL & \pm 0.31 & \pm 0.35 & \pm 0.42 & LSB \\
ENOB & 11.2 & 11.1 & 10.8 & Bit \\
SINAD & 68.9 & 68.8 & 66.9 & dB \\
SNR & 69.3 & 69.2 & 67.5 & dB \\
THD & -76.3 & -75.9 & -72.8 & dB \\
SFDR & 78 & 78 & 74 & dB \\
\hline \hline
\end{tabular}

Table 4.4: Summary of the AD41240 specification parameters extracted from the device characterization [10]. 
dose. The $\mathrm{V}_{b g}$ increased about $11.8 \%$ when a $10 \mathrm{kGy}$ dose was reached, but have not shown any further increase up to a total dose of $100 \mathrm{kGy}$. This consequently affects $\mathrm{V}_{\mathrm{cm}}$, gain and offset, which decreased their nominal values within $0.4 \%, 0.22 \%$, and $<0.7 \%$, respectively. On the other hand, this can be easily corrected during the calibration process [10].

\subsection{LVDS_RX adapters}

The AD41240 has LVDS differential output and FENIX ASIC place on the FE board has single ended LVCMOS input, hence a LVDS-to-LVCMOS adapter had to be involved for each VFE channel. Because it is not practical to carry differential signals through the connectors between the VFE card to the FE board, it was decided that the adapter circuit would be placed on the VFE PCB right next to the ADC chip. Since any radiation resistant adapters were not commercially available, a new ASIC named LVDS_RX, based on the CMOS $25 \mu$, was developed at IPN Lyon.

The adapter performance characteristics are summarized in Tab. 4.5 [16]. It consists of 8 parallel channels with differential inputs $\mathrm{V}_{\text {diff }}$ between -100 and $100 \mathrm{mV}$, each reserved for one bit from AD41240. Therefore two adapters, each with 7 active and one permanently passive channel, are needed for every VFE channel to transfer $12+2$ bits signals.

\begin{tabular}{|l|c|}
\hline \hline Parameter & Value \\
\hline $\mathrm{V}_{d d}$ & $2.5 \mathrm{~V}$ \\
DC current (7 active channels) & $<21 \mathrm{~mA}$ \\
Propagation delay & $<6 \mathrm{~ns}$ \\
Output signal rise and fall time & $\sim 2$ \\
Power consumption (at $40 \mathrm{MHz})$ & $100 \mathrm{~mW}$ \\
\hline \hline
\end{tabular}

Table 4.5: LVDS_RX performance specifications.

Test production program of all 210.000 manufactured pieces was performed by EDGETEK ${ }^{7}$ with a production rate of 20.000 adapters per month. The program, which includes a check of the DC, AC parameters and circuit functionality tests, was finished with a total production yield of $86 \%$ [6].

\subsection{Conclusion}

A brief description of the individual components of the VFE electronics, their electrical architecture and their functionality principles have been given in this chapter. The production procedures and necessary tests on the radiation tolerance have also been introduced. The final design of the VFE electronics with the new MGPA chip has been successfully completed in a very short time. The manufacturing process has been finished without any major difficulties a with high production yield and low price. In the next chapter, a screeming procedure and calibration of all the VFE cards are described and the main results are presented.

${ }^{7}$ EDGETEK, Les Ulis, France 



\section{Bibliography}

[1] E. Noah et al., "Total dose irradiation of a $0.25 \mu \mathrm{m}$ process," in $6^{\text {th }}$ Workshop on Electronics for LHC Experiments LEB 2000, Cracow, Poland, Sep 2000, pp. 555-559.

[2] J. Blaha et al., "Performance of CMS ECAL very front end electronics," in 12th Workshop on Electronics for LHC Experiments LECC 2006, Valencia, Spain, Sep 2006, pp. 174-178.

[3] M. Raymond et al., "The MGPA electromagnetic calorimeter readout chip for CMS," in $9^{\text {th }}$ Workshop on Electronics for LHC Experiments LECC 2003, Amsterdam, The Netherlands, Oct 2003, pp. 83-87.

[4] — "The MGPA electromagnetic calorimeter readout chip for CMS," IEEE Nuclear Science Symposium Conference Record, vol. 1, pp. 156-160, Oct 2004, 16-22 Oct. 2004, Rome, Italy.

[5] — - "The MGPA electromagnetic calorimeter readout chip for CMS," IEEE Transactions on Nuclear Science, vol. 52, no. 3, pp. 756-763, June 2005.

[6] D. Bon et al., "Rapport activité 2004 - 2005 - service electronique," IPN Lyon, Tech. Rep., 2005.

[7] "MGPA pre-production test results and mgpa testing production," http://indico.cern.ch/conferenceDisplay.py?confId=a044247, Oct 2004.

[8] "Status of the industrial MGPA chip tests," http://indico.cern.ch/conferenceDisplay. py? confId=a05341, Jan 2005.

[9] G. Minderico et al., "A CMOS low power, quad channel, 12 bit, 40MS/s pipelined ADC for applications in particle physics calorimetry," in $9^{\text {th }}$ Workshop on Electronics for LHC Experiments LECC 2003, Amsterdam, The Netherlands, Oct 2003, pp. 88-91.

[10] K. Kloukinas, S. Bonacini, and A. Marchioro, "Characterization and production testing of a quad 12 bit $40 \mathrm{Ms} / \mathrm{sec}$ A/D converter with automatic digital range selection for calorimetry," in $11^{\text {th }}$ Workshop on Electronics for LHC Experiments LECC 2005, Heidelberg, Germany, Sep 2005, pp. 241-245.

[11] K. Kostas and B. Sandro, The K-chip reference manual, Version 3B.1 Draft, CERN EP/CME and EP/MIC, Geneva, Jan 2004.

[12] Maxim Integrated Products, "INL/DNL measurements for high-speed analog-to-digital converters (ADCs)," Dallas Semiconductor, Application Note APP 283, Sep 2000. 
[13] G. Nicolas, "ABCs of ADCs - analog-to-digital basics," National Semiconductor, Analog University Rev 3, June 2006.

[14] Maxim Integrated Products, "Histogram testing determines DNL and INL errors," Dallas Semiconductor, Application Note APP 2058, May 2003.

[15] — _ "Defining and testing dynamic parameters in high-speed ADCs, part 1," Dallas Semiconductor, Application Note APP 728, Feb 2001.

[16] "Buffer production testing," http://indico.cern.ch/conferenceDisplay.py?confId=a041933, May 2004. 


\section{Chapter 5}

\section{VFE Calibration and performance}

\subsection{Introduction}

VFE calibration and performance test were carried out as a part of the readout electronics QA program during which the systems for burn-in and precise calibration of the VFE boards were developed and successfully used at IPN Lyon. A brief description of these systems and the main obtained results are given in this chapter.

\subsection{Production program}

The test program of the almost $\sim 16,000 \mathrm{VFE}$ cards consists of an optical inspection, power-on test, and burn-in which is followed by a complete calibration and characterization of each VFE channel. As the other components of the CMS all the VFE cards are fully identified by a bar-code number which allows its unique identification in the CMS and LHC. All relevant test results are stored in a data base. During the construction of the calorimeter the geographical identification of each card is registered associating the VFE calibration data with a given read-out channel, crystal, and APD or VPT. The production program is composed of four consecutive tests:

- Automatic Optical Inspection (AOI) of the card by the manufacturer ASCOM, Schweiz AG.

- Power-on test, including a limited functional test by the manufacturer and at the reception at CERN performed by ETH Zürich.

- Burn-in test for three days at a temperature of $60^{\circ} \mathrm{C}$ at IPN Lyon.

- Calibration and characterization of all the VFE cards at IPN Lyon.

\subsection{Automatic optical inspection}

The Automatic Optical Inspection (AOI) test consists of pattern recognition of the cards. It is performed on the all manufactured cards to prevent systematic production mistakes. It checks the presence of all components, active and passive, and verifies the soldering quality. The AOI test is developed by the manufacturer, using a specialized automatic machine [1]. 


\subsection{Power-on test}

The power-on test $[2,3]$ is the first electrical test of the VFE card and was performed twice, once at the manufacturer before delivery and after reception in the ECAL electronics integration center at CERN. Therefore three identical systems, two placed at the manufacturer and one at CERN were used.The power-on test system, of which a schematic block diagram is depicted in Fig. 5.1 [3], is composed of a programmable power supply, a Power On Test Board (POTB) housing a Xilinx FPGA, and a NI DAQ card. Each VFE card is identified by a barcode reader and the whole system is controlled by a master PC that is connected with POTB via RS232 interface.

Out of the total 12,887 barrel VFE cards which have been verified with the CERN power-on test, only $1 \%$ (99 pieces) of VFE cards have not matched the specification criteria and have been consequently removed from the production program. The power-on test focused on the two main aspects, the simple functional test of each channel, and slow control of the VFE and on its power consumptions.

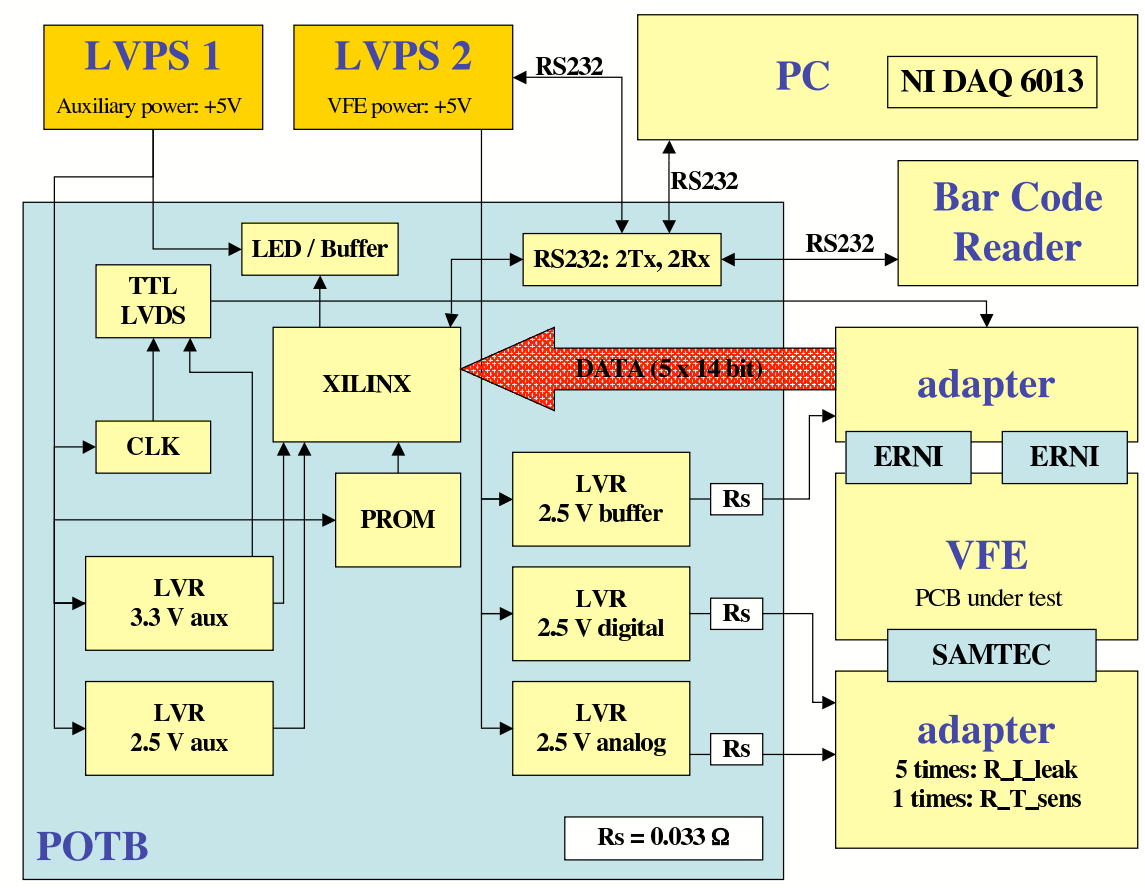

Figure 5.1: Block diagram of the power-on test system showing Power On Test Board (POTB) and plugged VFE card under test [1].

\subsubsection{Functional tests}

A functional test is performed by use of the Xilinx FPGA which processes digitalized data at $40 \mathrm{MHz}$. It reads simulated APD leakage currents and crystal temperatures from the DCU chip, sets the MGPA and ADC modes, and records and analyzes the data generated by the MGPA 
test pulse facility.

In order to check the correct functionality of each VFE channel, one MGPA test pulse $(4.1 \mathrm{pC})$ is applied to every gain channel and a subsequent ADC digital output is measured. All the required parameters: pedestal, noise, amplitude pulse height, and relative gain are measured and compared with specification values. For each gain, VFE cards with pedestal value greater than 1,000 ADC counts or with pulse height outside the specification limits [3] have been considered as bad ones and have been rejected from the production program. The VFE cards which fulfill this selection criteria show distributions with a clear Gaussian shape. Mean values and corresponding dispersions are summarized in Tab. 5.1. The small dispersion indicates good production uniformity. One have observed a large dispersion for pedestal distribution. This dispersion is due to the fact that the DAC pedestal were not set to a specific value as in case of the calibration tests of the VFE cards.

Due to the construction solution of the power-on system, which was designed only for a fast test of the basic VFE functions, the noise could not be evaluated accurately and it was decided to leave the identification of possibly noisy channels for the consecutive calibration measurement in IPN Lyon, where all the channels are precisely characterized.

\begin{tabular}{|ll|ccc|}
\hline \hline & & Gain x1 & Gain x6 & Gain x12 \\
\hline Pulse height & Mean [ADC counts] & 262.5 & 1446 & 2813 \\
& RMS/mean [\%] & 1.51 & 1.39 & 1.55 \\
Pedestal & Mean [ADC counts] & 248.3 & 283.4 & 441.9 \\
& RMS/mean [\%] & 21.32 & 26.58 & 22.74 \\
\hline \hline
\end{tabular}

Table 5.1: Pulse height and pedestal values for all tested VFE barrel cards which fulfill specifications measured for the free gains 1,6 , and 12 .

\subsubsection{Power consumptions}

The VFE card requires three separate voltage supply: $2.5 \mathrm{~V}$ analog to power the MGPA chip and analog part of the ADC, and $2.5 \mathrm{~V}$ digital to power the digital part of the ADC, and the third one that is dedicated for the LVDS to single ended CMOS buffer LVDS-RX and buffer LVDSbuff. These three input and output voltages of the power supply are measured by the NI DAQ card. The corresponding currents are determined by measuring voltage drop over calibrated shunt resistances with a nominal value of $0.033 \Omega$ ( $1 \%$ precision).

Figure 5.2 [3] shows the distributions of voltages and currents which were measured separately for: the analog and digital part of the VFE card as well as for buffers which includes both LVDSRX and LVDS-buff. The mean values with corresponding dispersions are listed in Tab. 5.2. The distribution of the power supply voltages has two peaks due to the variation of the power supply voltages during the testing period. It was assumed that the left hand tail in the distribution of the buffer voltage are not due to malfunction of the VFE, but because of an error in the device measuring the buffer voltage. The other distributions have a Gaussian shape as expected.

Table $5.3[3]$ summarizes the expected and measured currents for the individual component and for a complete VFE card. The measured values are given as a mean value over 12,887 barrel VFE cards measured by the power-on system. Since the system measures the analog, digital, 

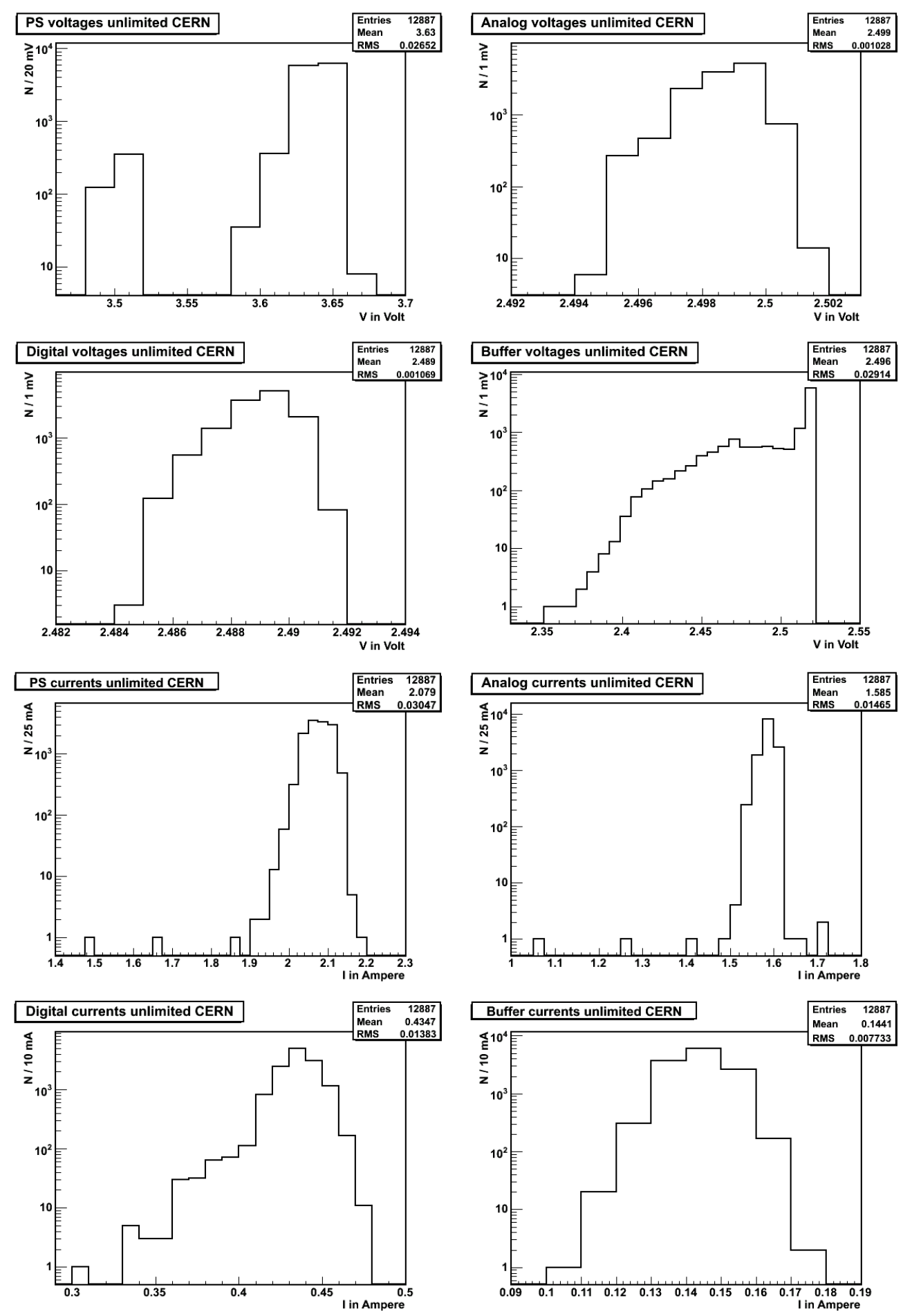

Figure 5.2: Distributions of the power supply, analog, digital and buffers voltage and currents measured for all 12,887 tested barrel VFE cards [3]. 


\begin{tabular}{|l|cc|}
\hline \hline Voltage & Mean [V] & RMS/mean [\%] \\
\hline PS & 3.63 & 0.73 \\
Analog & 2.499 & 0.041 \\
Digital & 2.489 & 0.043 \\
Buffer & 2.496 & 1.17 \\
\hline Current & Mean[A] & RMS /mean [\%] \\
\hline PS & 2.07 & 1.47 \\
Analog & 1.585 & 0.92 \\
Digital & 0.435 & 3.18 \\
Buffer & 0.144 & 5.37 \\
\hline \hline
\end{tabular}

Table 5.2: The mean values of the power supply: analog, digital, buffers voltages and currents measured for all 12,887 tested barrel VFE cards.

buffers voltages and currents separately, the power consumptions of each individual components specified cannot be specified. Also the currents of the LVDS-buff can only be estimated to be $10 \mathrm{~mA}$ each. Therefore, the resulting currents of the ADC and the LVDS-RX were calculated using this estimation. The total mean value of the $\mathrm{VFE}$ current is $2.17 \mathrm{~A}$, which corresponds to 5.5 Watts which is fully in accordance with CMS specifications.

\begin{tabular}{|l|c|c|c|c|}
\hline \hline Component & \# per VFE & $\mathrm{I}[\mathrm{mA}]$ analog & $\mathrm{I}[\mathrm{mA}]$ digital & $\mathrm{I}[\mathrm{mA}]$ buffers \\
\hline MGPA & 5 & 232 & & \\
AD41240 & 5 & 101.6 & $110.8(85)$ & \\
LVDS-RX & 10 & & & $50(13.4)$ \\
LVDS-buff & 1 & & & 10 \\
DCU & 1 & & 10 & \\
\hline VFE & 1 & $1668(1585)$ & $564(435)$ & $510(144)$ \\
\hline \hline
\end{tabular}

Table 5.3: Expected (measured) values of the analog, digital and buffers currents for each individual component and for a fully functional barrel VFE card. Measured values are the mean values over all the VFE cards measured in power-on test.

\subsection{Burn-in}

\section{Burn-in overview}

The burn-in technique [4] is a widely used engineering method for screening out early failures in a group of components or systems prior to their introduction into general service. It is based on the fact that a disproportionate amount of failures occur in the early period of their service.

This behavior is described by the time-to-failure model of the reliability theory which assumes that the components' strength deteriorates with time and that the weak components deteriorate faster than the strong components. This model can be then mathematically de- 
scribed by the probability time-to-failure density function $f(t)$ computed from the number of component failing per unit of time, and corresponding to the feature distribution function or cumulative distribution function $F(t)$ which quantifies the probability of failure. It was proved that the distribution of the probability density function follows a bimodal pattern with the main population centered around expected value of the component lifetime and smaller population which belongs to freak failure components. Another useful quantity is the hazard rate $h(t)$ or so called failure rate that is the frequency with which the component fails and is computed as $h(t)=f(t) / R(t)$, where $R(t)=1-F(t)$ is the probability of survival, i.e. the reliability at time $t$. The failure rate versus operational time is well-know as a bathtub curve (see Fig. 5.3) which shows high failure rate at the beginning of the curve which drops as the weak components fail early in their lifetime and stays nearly constant with the main contribution coming from random failures. The time period when the failure rate is constant characterizes a useful lifetime of the component. Finally, the failure rate increase steeply due to wear-out failures as the product exceeds its design lifetime. The reason of the large number of the early failures is attributed to the fact that variability in the production processes, which also includes screening and ultimate handing of the product, introduces weaknesses in some parts and not in others.

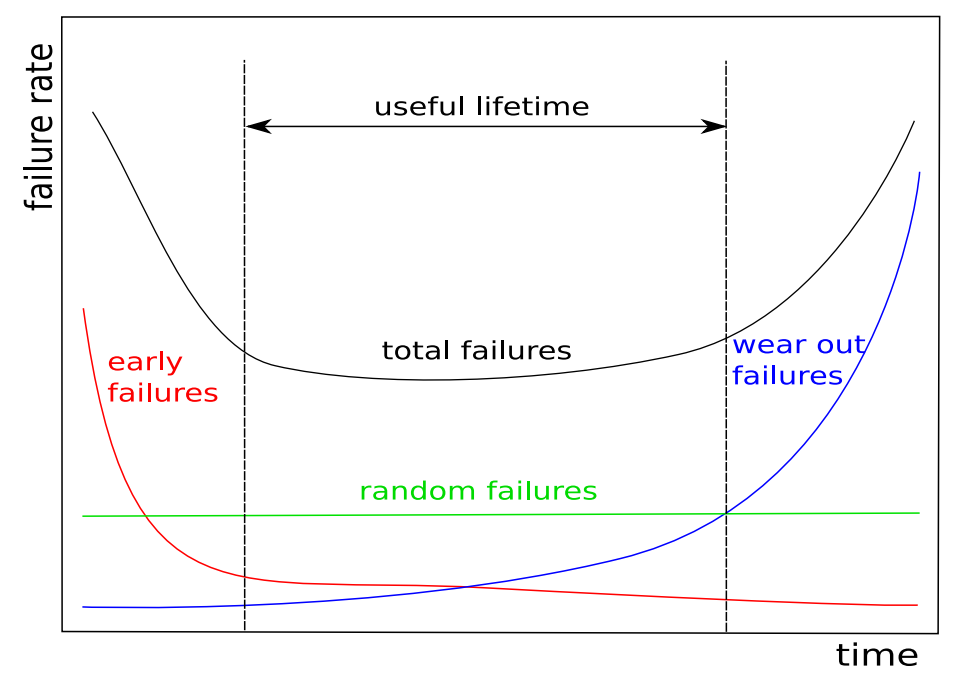

Figure 5.3: The bathtub curve.

Other types of early failures are those pertaining to infant mortality, created during the manufacturing process of the components into a more complex system. Contrary to the freak failures, which have the same failure mechanism as the main population, but occur earlier in time, the infant mortality failures can be caused by different types of defects. Therefore, the complete burn-in process to eliminate all early failures needs to be carried out in two steps. In the first step, the freak failures can be screened out by components burn-in and in the second step the infant mortality failures can be screened out by a system burn-in.

\section{Accelerated burn-in}

Acceleration of the burn-in test is used to effectively find freak and infant mortality failures in a reduced time period based on the fact that the reliability of electronic components decreases 
when increasing temperature. This can be described by the Arrhenius model. In this model, the strength degradation leading to component failure is governed by chemical and physical processes with a reaction rate given by equation [4]:

$$
r=A \exp \left(-E_{A} / k T\right)
$$

where $A$ is a constant, $E_{A}$ is the activation energy $(\mathrm{eV}), k$ is the Boltzmann's constant $(8.617343 \pm$ $0.000015) \times 10^{-5} \mathrm{eV} \cdot \mathrm{K}^{-1}$ ), and $T$ is the absolute temperature $(\mathrm{K})$. The activation energy $E_{A}{ }^{1}$ is determined experimentally. For instance if $E_{A}=1 \mathrm{eV}$, which is the widespread use value (but may differ for some manufactures), and the burn-in temperature is around $65^{\circ} \mathrm{C}$ then the acceleration factor relative to a normal operation temperature $25^{\circ} \mathrm{C}$ is equal to 100 . In other words, the early failures occur after a period 100 times shorter than if observed in normal operational conditions.

\subsubsection{VFE burn-in procedure}

It was decided to perform an accelerated burn-in test for the fully assembled VFE cards produced after the power-on test and prior to calibration and characterization measurements. The burnin procedure was chosen to identify infant mortality and freak failures, therefore the burn-in consists of a sufficient heat soaking period of 72 hours at a temperature of $60^{\circ} \mathrm{C}$. Due to the large quantity of VFE cards, the stress was also put on the production burn-in rate, where at least $600 \mathrm{VFE}$ cards had to be burned out during one week.

The burn-in system, of which the functional block schema is depicted in Fig. 5.4 and photographs of the system during its manipulation are shown in Fig. 5.5, was designed to handle up to $300 \mathrm{VFE}$ cards simultaneously. A burn-in temperature of $60^{\circ} \mathrm{C}$ is reached within an hour after the beginning of the test and is kept constant by a temperature sensor Pt100 which monitors it with $0.1 \%$ precision. To avoid an unwanted temperature gradient in the hot zone, continuous air circulation is provided by an electrical fan. To be as close to the VFE final operation conditions as possible, the cards are powered with both $2.5 \mathrm{~V}$ analog voltage and $2.5 \mathrm{~V}$ digital voltage and also receive the $40 \mathrm{MHz}$ clock. No input signal, as in the case of power-on and calibration tests, is injected and their inputs are simply loaded with $180 \mathrm{pF}$ capacitors. An active motherboard monitors the current consumption of each card and checks if it remains in a predefined window, i. e. between 0.7 and $3 \mathrm{~A}$. In case of under-consumption, a digital signal is stored in a single bit local register. In case of over-consumption the card is immediately (less than $100 \mu \mathrm{s}$ ) disconnected from the power supply and a digital signal is stored in a second single bit local register. Periodically, a PC workstation running labview application checks these registers and logs the status of the VFE card. Thus a report file showing continuous behavior of the card is available at the end of the burn-in procedure. Current status of the burn-in process is regularly checked via remote connection to the master PC by the person in charge of the test.

The first burn-in was performed with a pre-serie of $420 \mathrm{VFE}$ cards and was intended to verify both the quality of the card and the VFE card production program itself, i. e. in case of burn-in if the procedure is set correctly. There is one delicate question, especially important in the case of accelerated burn-in tests:" Do we degrade the surviving products with the burn-in test?" The correct answer is yes, the components are degraded somewhat, but the extent of degradation is

\footnotetext{
${ }^{1}$ The activation energy is connected primarily with a failure mechanism (oxide defects, contamination, electron migration, etc) in semiconductors. Its value is determined experimentally by observing time-to-failure of different components at different temperatures.
} 


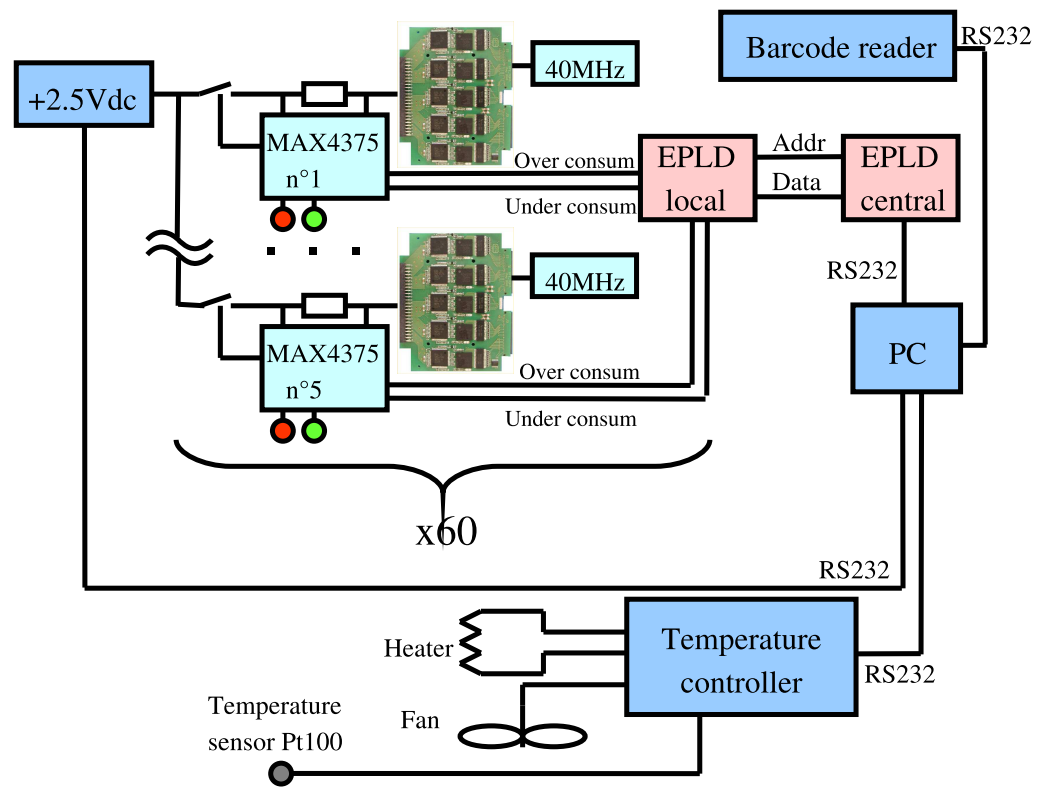

Figure 5.4: Block diagram of the burn-in system showing two (out of 300) VFE cards under test.
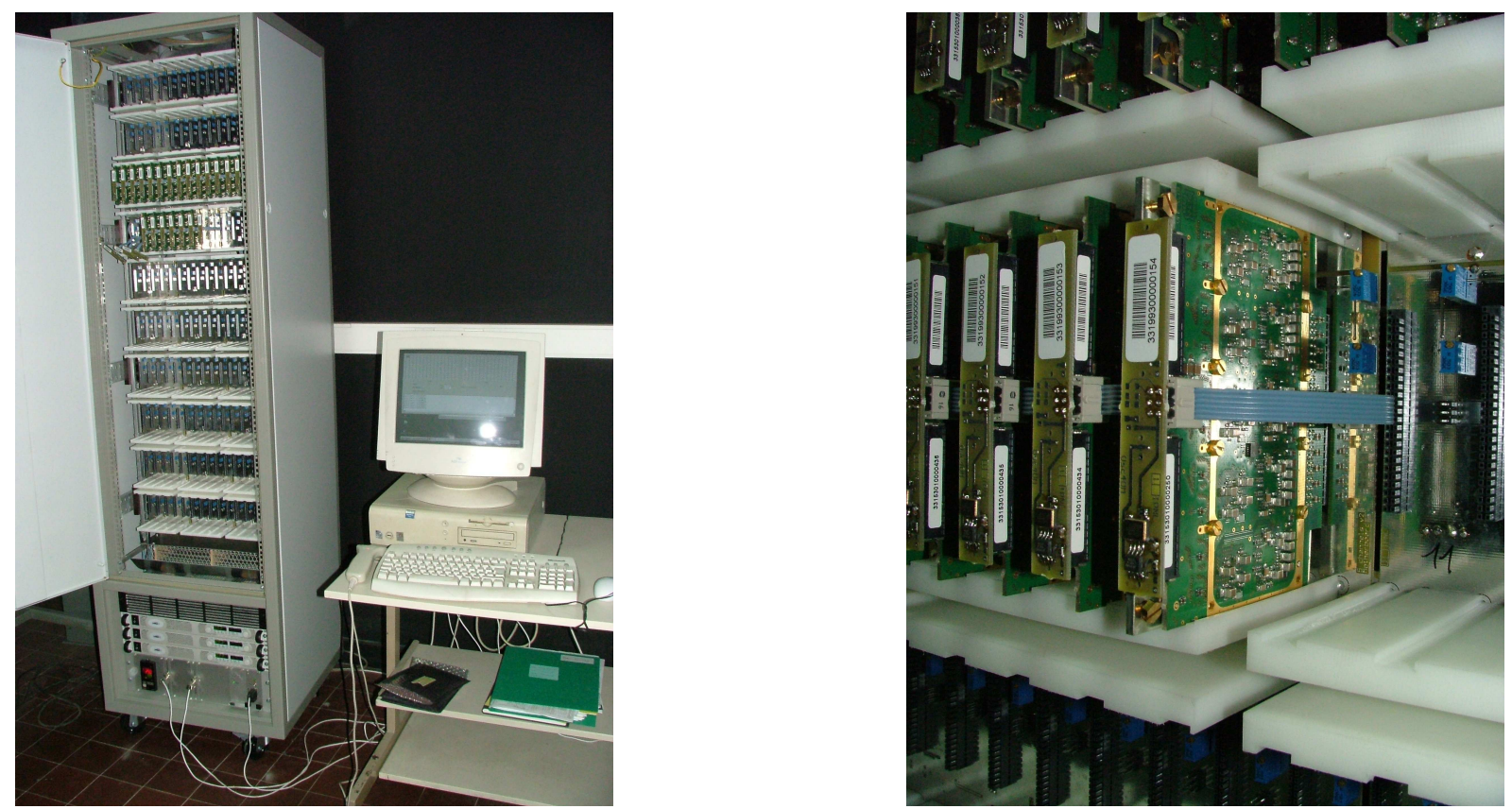

Figure 5.5: Left: The set-up of the burn-in system at IPN Lyon. Other 150 VFE cards can be plugged in the back of the system. Right: Detailed view of the plugged VFE cards. 
usually not severe. To verify if there is any influence of the burn-in, all possible characteristics of the pre-production cards were evaluated twice, before and after burn-in test. The results of those tests proved that there is no visible sign of distortion in measured parameters.

The burn-in test for 12,788 barrel and 3000 end-cap VFE cards has been completed. Only three barrel cards have failed the burn-in test. These cards were also subsequently calibrated and fully characterized. Since all their parameters have been found within specifications, it was considered that they didn't failed the burn-in test for reason of early failures but due to accidental malfunction of the burn-in system. It has to be noted that the burn-in test where no infant mortality or freak failures were found is not at all uncommon. It indicates a very high quality of the VFE card with careful planning of their production and a high effective screening program which were applied prior to the burn-in test and where the main portion of the defected VFE cards were already recognized and rejected from the production queue.

\subsection{VFE calibration and performance}

The calibration test $[2,5-7]$ is intended to check all the operational parameters of the VFE card and builds a calibration database for each channel which will serve for the first calibration of all the channels of the ECAL. The physics requirements on the testing of VFE electronics as stated by CMS collaboration [8] in 2004 determining the main aims of the laboratory calibration, are listed below with values of the maximum allowed dispersions between channels in order to fulfil desired ECAL physics performance:

- Absolute calibration of the three gains for each channel in $\mathrm{ADC} / \mathrm{pC}$ with dispersion less than $5 \%$.

- Relative calibration between channels. In order to fulfil the target precision for intercalibration using laboratory measurement (4\%), the required dispersion has to be better than $2 \%$.

- Gain ratios between the gain ranges: gain 12/gain 6 and gain 12/gain 1 . There are no strict requirements, because it is expected that the gain ratio values will be determined precisely with the laser system. Due to the cross-checking purpose the dispersion in gain ratios should not exceed $2 \%$.

- Linearity studies. In order to accurately evaluate the linearity of the VFE electronic, the injected charge $\Delta Q / Q$ have to be know with a precision better than $0.5 \%$.

\subsubsection{Test bench}

A complete test bench has been designed at IPN Lyon. Up to 6 test boards can be installed in a 19 inche $6 \mathrm{U}$ crate. A schematic block diagram of the system functionality is shown in Fig. 5.6 and a photograph of the actual test board with a VFE card under the test and the whole calibration system at IPN Lyon are shown in Fig. 5.7. Each test board is divided into an analog and a digital part. The analog part is composed of an analog power regulation for the VFE $+2.5 \mathrm{~V}$ analog, a charge generation (buffering and derivation of the input square pulse coming from the attenuator), leakage current generation (DAC and voltage to current converter) and thermistor emulation, which is in fact a digitally programmable potentiometer. The digital part of the board is composed of a digital power regulation for the VFE $+2.5 \mathrm{~V}$ digital, VFE 


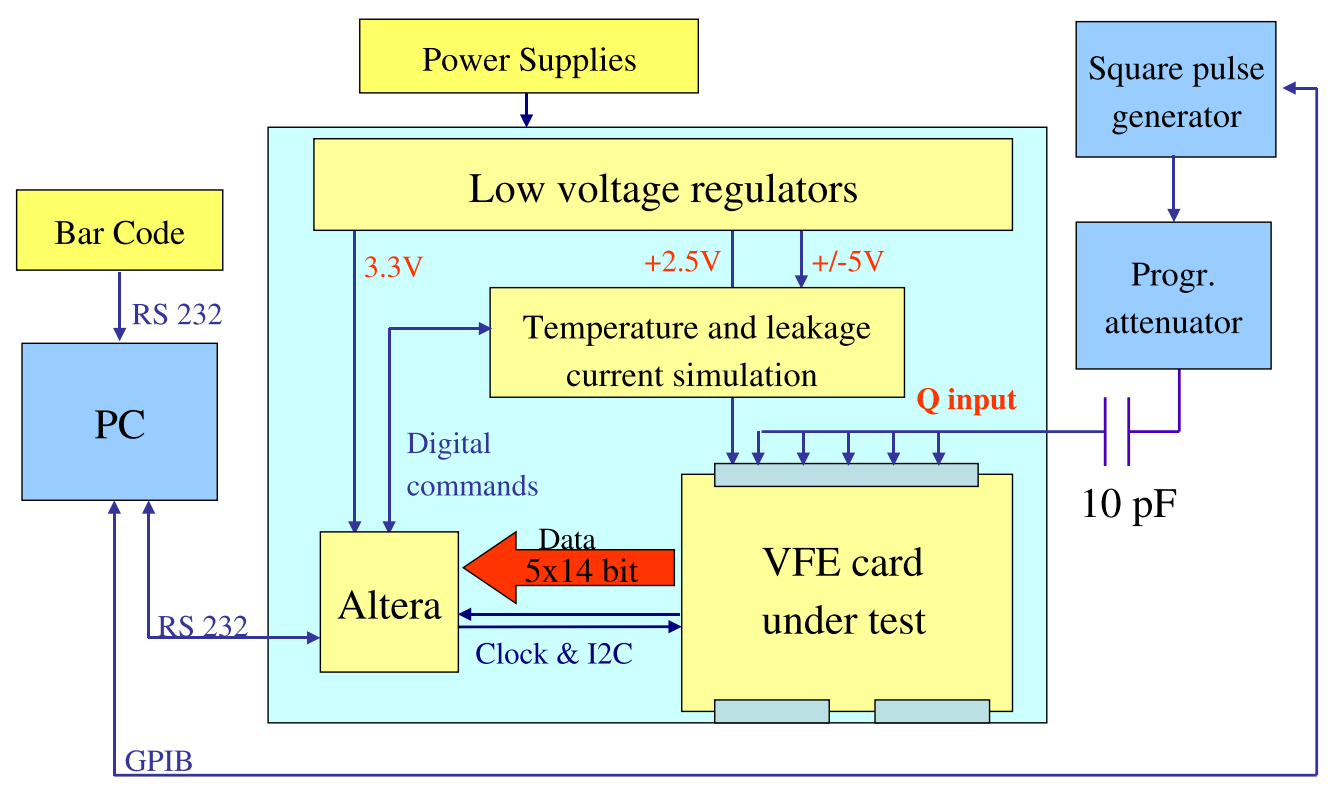

Figure 5.6: Schematic block diagram of the calibration test system developed and used at IPN Lyon and the test board with its internal main functionality parts.
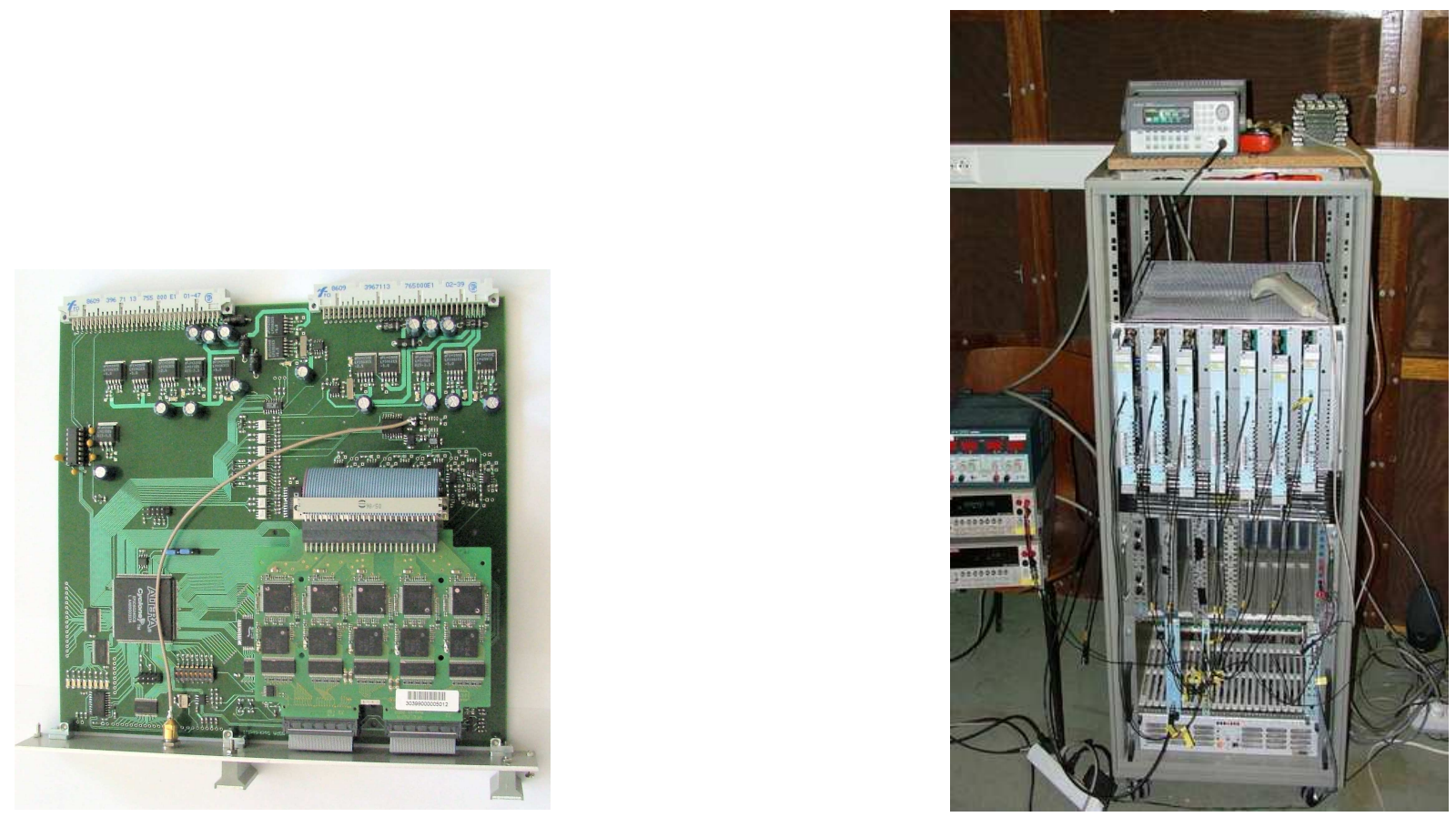

Figure 5.7: Photograph of the test board card (right) and the calibration system (left) for the VFE cards developed and used at IPN Lyon. 
data recorder and one Altera FPGA that is in charge of the communication with the master PC, the stimuli generators and the VFE card (see Fig. 5.6).

\subsubsection{Calibration procedure}

Due to the large amount of the VFE cards $(\sim 16,000)$ which have to be tested, the same test system was build for four regional centers: IPN Lyon, INFN Turin, ETH Zürich (the system placed at CERN) and UC Nicosia. However, for homogeneity reasons, only the system in Lyon has been in use for the calibration.

The different steps of the calibration test, for each VFE card and channel, are the following:

- Verify the communication with the chips via the $\mathrm{I}^{2} \mathrm{C}$ bus.

- Set the pedestal and measure the electronic noise at the three different gains.

- Vary the charge pulse injection over the full dynamic range, measure the response in ADC counts (12 bit) and verify the linearity.

- Verify the good behavior of the test pulse functionality of the MGPA.

Furthermore the following characteristics are:

- Simulate the leakage current of the APDs and compute the corresponding linearity.

- Test the temperature read-out channel on its complete dynamic range.

In order to check the linearity, a set of pulses of a precise charge are injected into the VFE card. The system consists of an Agilent square pulse generator with a pulse height of $5 \mathrm{~V}$, a rise- and fall-time of $25 \mathrm{~ns}$ and a voltage attenuator. This allows to obtain different voltage values that are applied on a calibrated $10 \pm 0.01 \mathrm{pF}$ capacitor which produces charges in a range from 0 up to $40 \mathrm{pC}$ for the barrel and up to $9 \mathrm{pC}$ for the endcap VFE card. Different values of charge are injected into the VFE card and a digital output of the card is recorded over the above mentioned dynamic range for all three gains and five channels. The main specification limits and typical values of corresponding parameters for barrel and endcap VFE card are summarized in Tab 5.4.

The stability of the leakage current is tested using a current source which simulates a variation of the leakage current in a range between $0-200 \mathrm{nA}$ to the input of the VFE. The data are collected by the DCU chip placed on the VFE and are sent to the data acquisition system via the $\mathrm{I}^{2} \mathrm{C}$ interface. For the temperature read-out channel of a crystal, the test board uses a digital potentiometer that changes its value to simulate a temperature variation of the crystals. The DCU reads and sends the data to the data acquisition system. All data are sent to an ALTERA FGPA placed on the test board and then via a RS 232 bus to the master PC (see Fig. 5.6) where they are stored in a data base.

\section{Calibration curve}

The calibration curve describes the relation between the analog input on the VFE channel, which can be an injected calibration charge as well as the energy deposited in the crystal by an impinging particle, and the digital output of the VFE card measured in ADC counts.

Every $25 \mathrm{~ns}$ which corresponds to the $40 \mathrm{MHz}$ LHC running frequency one sample is recorded. Fig. 5.8 shows the measured output pulse shapes in ADC counts for a typical barrel (top) and 


\begin{tabular}{|l|c|ccc|ccc|}
\hline \hline & & \multicolumn{3}{|c|}{ Barrel } & \multicolumn{3}{c|}{ End-cap } \\
\hline Parameter & Gain & Min & Typical & Max & Min & Typical & Max \\
\hline Slope & 1 & 55 & 65 & 75 & 270 & 310 & 350 \\
{$[$ ADCcounts $/ p C]$} & 6 & 310 & 350 & 395 & 1450 & 1620 & 1800 \\
& 12 & 620 & 685 & 760 & 2800 & 3120 & 3500 \\
\hline Offset & 1 & -12 & -6 & 2 & -35 & -20 & -5 \\
{$[$ ADCcounts $]$} & 6 & -8 & 2 & 12 & -35 & -9 & 20 \\
& 12 & -8 & 2 & 12 & -35 & -6 & 20 \\
\hline Noise & 1 & 0 & 0.5 & 2 & 0 & 0.6 & 3 \\
{$[$ ADCcounts $]$} & 6 & 0 & 0.7 & 5 & 0 & 2.9 & 10 \\
& 12 & 0 & 1 & 10 & 0 & 5.9 & 15 \\
\hline Relative gain & $12 / 1$ & 9.5 & 11 & 12 & 9.5 & 11 & 12 \\
& $12 / 6$ & 1.8 & 2 & 2.1 & 1.8 & 2 & 2.1 \\
& $6 / 1$ & 5 & 5.5 & 6 & 5 & 5.5 & 6 \\
\hline Non Linearity $[\%]$ & all gains & 0 & 1 & 2 & 0 & 1 & 2 \\
\hline Pedestal & all gains & 0 & 160 & 1000 & 0 & 160 & 1000 \\
{$[$ ADCcounts $]$} & & \multicolumn{7}{|c|c|c}{} \\
\hline \hline
\end{tabular}

Table 5.4: The specification values for VFE cards. The VFE card with measured values outside of these specification limits was considered as bad and rejected from the production queue. The slope and offset are related to the linear fit (see Par. Calibration curve and Fig. 5.10) of the VFE response versus injected charge.

end-cap (bottom) VFE card. A peak maximum is held at a constant time and fitted for each injected charge, by the use of five points around maximum, with an analytic function of form [9]:

$$
f(t)=\left\{\begin{array}{ll}
\left(\frac{t-T_{0}}{\beta}\right)^{\alpha} \exp \left(-\alpha \frac{t-T_{0}-\beta}{\beta}\right) & \text { if } t>T_{0} \\
0 & \text { otherwise }
\end{array},\right.
$$

where $T_{0}$ is a time value for the beginning of the fit curve and the parameters $\alpha$ and $\beta$ describe the shape of the pulse. A pedestal value, which is set to be around 160 ADC counts by $\mathrm{I}^{2} \mathrm{C}$ bus and is estimated for each injected charge separately using a set of pre-samples (the first 20 samples), is subtracted from the pulse amplitude.

In order to check the linearity, a set of pulses of precise charges are injected into the VFE card and a digital output of the card is recorded over the full dynamic range for all three gains and five channels. The calibration ranges of the injected charges were chosen to be below saturation for all three gains and to provide sufficient amount of points for the calibration as well as for linearity studies. Therefore 8, 9, and 10 injected charges are used, see Tab. 5.5. The charge steps are not linearly spaced because of the logarithmic nature of the attenuator.

The attenuator, type $50 \mathrm{P}-1202^{2}$, is a programmable attenuator that provides attenuation in a range from 0 up to $63 \mathrm{~dB}$ with an accuracy of $2 \%( \pm 0.3 \mathrm{~dB})$. Since such a precision is not sufficient for the VFE calibration, for which the knowledge of the delivered voltage pulses

\footnotetext{
${ }^{2} \mathrm{JFW}$ Industries
} 

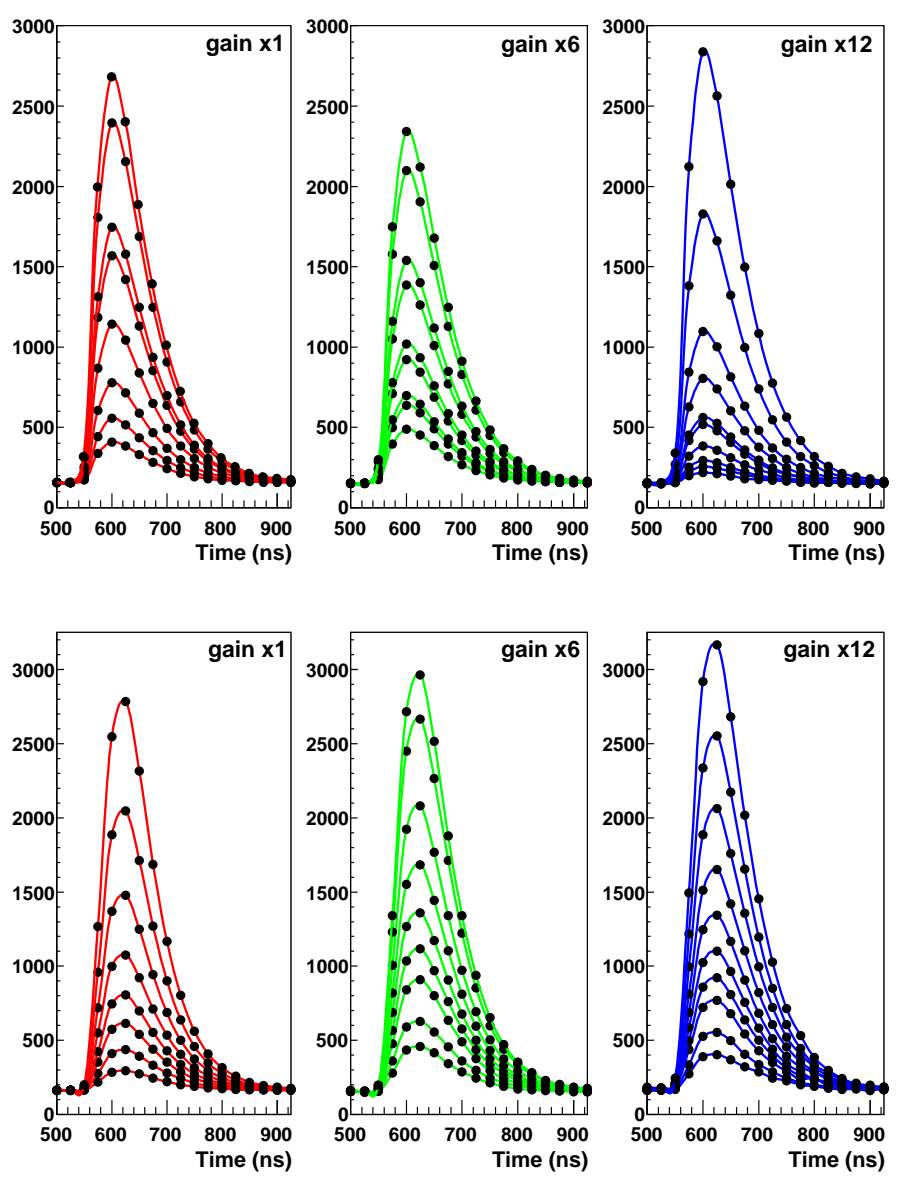

Figure 5.8: Typical injected charge pulses as VFE output in ADC counts for barrel (top) and endcap VFE (bottom) cards for gain 1, 6, and 12. The lines are only to guide the eye.

\begin{tabular}{|l|cccccccccc|}
\hline \hline Barrel & 1 & 2 & 3 & 4 & 5 & 6 & 7 & 8 & 9 & 10 \\
\hline Gain 1 & $\mathbf{4}$ & 6.32 & 9.85 & 15.56 & 22.26 & 25.02 & 35.15 & 39.51 & - & - \\
Gain 6 & 0.96 & 1.4 & 1.58 & 2.21 & 2.49 & 3.56 & $\mathbf{4}$ & 5.62 & 6.32 & - \\
Gain 12 & 0.01 & 0.16 & 0.21 & 0.34 & 0.54 & 0.61 & 0.96 & 1.4 & 2.49 & $\mathbf{4}$ \\
\hline Endcap & 1 & 2 & 3 & 4 & 5 & 6 & 7 & 8 & 9 & 10 \\
\hline Gain 1 & $\mathbf{0 . 4 8}$ & $\mathbf{0 . 9 8}$ & 1.6 & 2.24 & 3.17 & 4.52 & 6.41 & 8.84 & - & - \\
Gain 6 & 0.2 & $\mathbf{0 . 4 8}$ & $\mathbf{0 . 4 8}$ & 0.62 & 0.78 & $\mathbf{0 . 9 8}$ & 1.23 & 1.6 & 1.78 & - \\
Gain 12 & 0.08 & 0.12 & 0.2 & 0.24 & 0.31 & 0.38 & $\mathbf{0 . 4 8}$ & 0.62 & 0.78 & $\mathbf{0 . 9 8}$ \\
\hline \hline
\end{tabular}

Table 5.5: Experimental values of the injected charges in $\mathrm{pC}$ for the barrel and endcap VFE cards for gains 1,6 , and 12 . 
is crucial, a precise calibration of attenuator is needed. The attenuator was calibrated by a precise digital multimeter Keythley KE 2000, which allows to measure voltages with a precision of $0.02 \%$.

The voltage pulses were measured on the output of the attenuator 50 times with a step of $1 \mathrm{~dB}$. The average values were normalized to an input voltage of $5 \mathrm{~V}$ in order to determine the attenuation factors. These factors were then compared to theoretical ones to determine correction constant for each attenuation. Thus, the obtained precision of the attenuator is less than $0.05 \%$. The relative difference between measured and theoretical attenuation as a function of attenuation in displayed in Fig. 5.9, where one can see how the individual attenuation values reflect the internal structure of the attenuator circuits. The calibration of the attenuator was checked periodically during the whole VFE calibration and shows extremely stable behaviour.

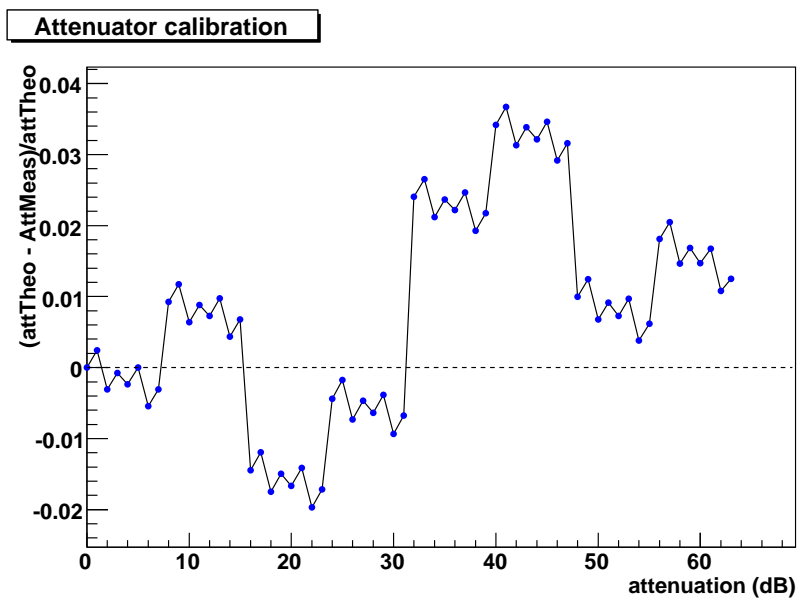

Figure 5.9: Relative difference between the theoretical and measured attenuation for each $1 \mathrm{~dB}$ step of the attenuator. The internal structure of the attenuator, where every attenuation is made as a combination of $1,2,4,8,16$, and $32 \mathrm{~dB}$ attenuation units.

Linear dependence of the ADC VFE response on injected charges is shown for all three gains in Fig 5.10. The saturation levels, which are not reached, are around $60 \mathrm{pC}$ and $16 \mathrm{pC}$ for barrel and endcap VFE cards, respectively (see Chap.5.6.8). Several charges were chosen as overlap points to verify a good link between different gains and are highlighted in Tab. 5.5 using bold font.

\subsubsection{Calibration results}

Measurements of the calibration curves, shown for one channel in Fig. 5.10, have been completed for all VFE cards needed for the barrel and endcaps. Parameters of a linear least square fit of the calibration curve describe the main properties of each gain channel. Average values of the slopes, which represent the gains, measured in ADC counts per $\mathrm{pC}$ over all calibrated channels are listed together with the corresponding dispersions in Tab. 5.6. Very small differences $(\sigma /$ mean $\sim 1 \%$ ) among measured channels prove the high quality and homogeneity in the VFE board production. Slope distributions of the injected charge versus pulse height in ADC counts/pC for all the barrel and endcap VFE cards are displayed in Fig. 5.11. The offset value for all barrel 
and endcap VFE cards are displayed in Fig. 5.12. One can see from these plots the mean offset values are not zero as would be in ideal case. This is due to non linearity of the whole electronic chain for small charges. The mean value of the offset as well as dispersions are much more larger and less gaussian for endcap cards than for barrel cards which is caused by different external input components which set the different dynamic range for those cards. The deformation from the gaussian distribution in the gain 1 in endcap can be explained by the non-linearity at small charges (see Fig. 5.19).
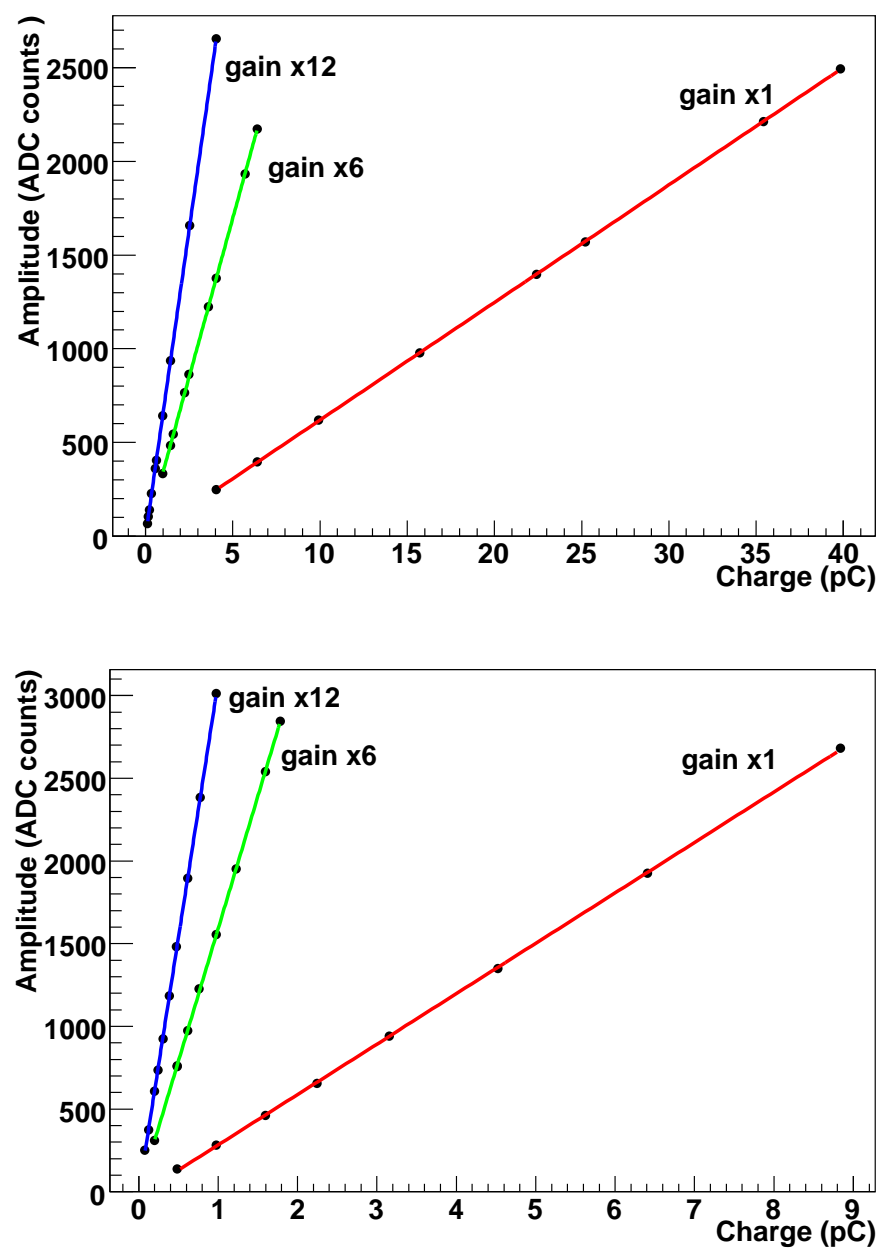

Figure 5.10: VFE outputs in ADC counts as a function injected charge for a typical channel of the barrel (top) and endcap VFE (below) cards representing calibration curves for the three gains 1,6 , and 12. A charge of $4 \mathrm{pC}$ for barrel and 0.48 or $0.98 \mathrm{pC}$ for endcap VFE cards are common for all three gains and allows to perform a direct gain ratio measurement. 

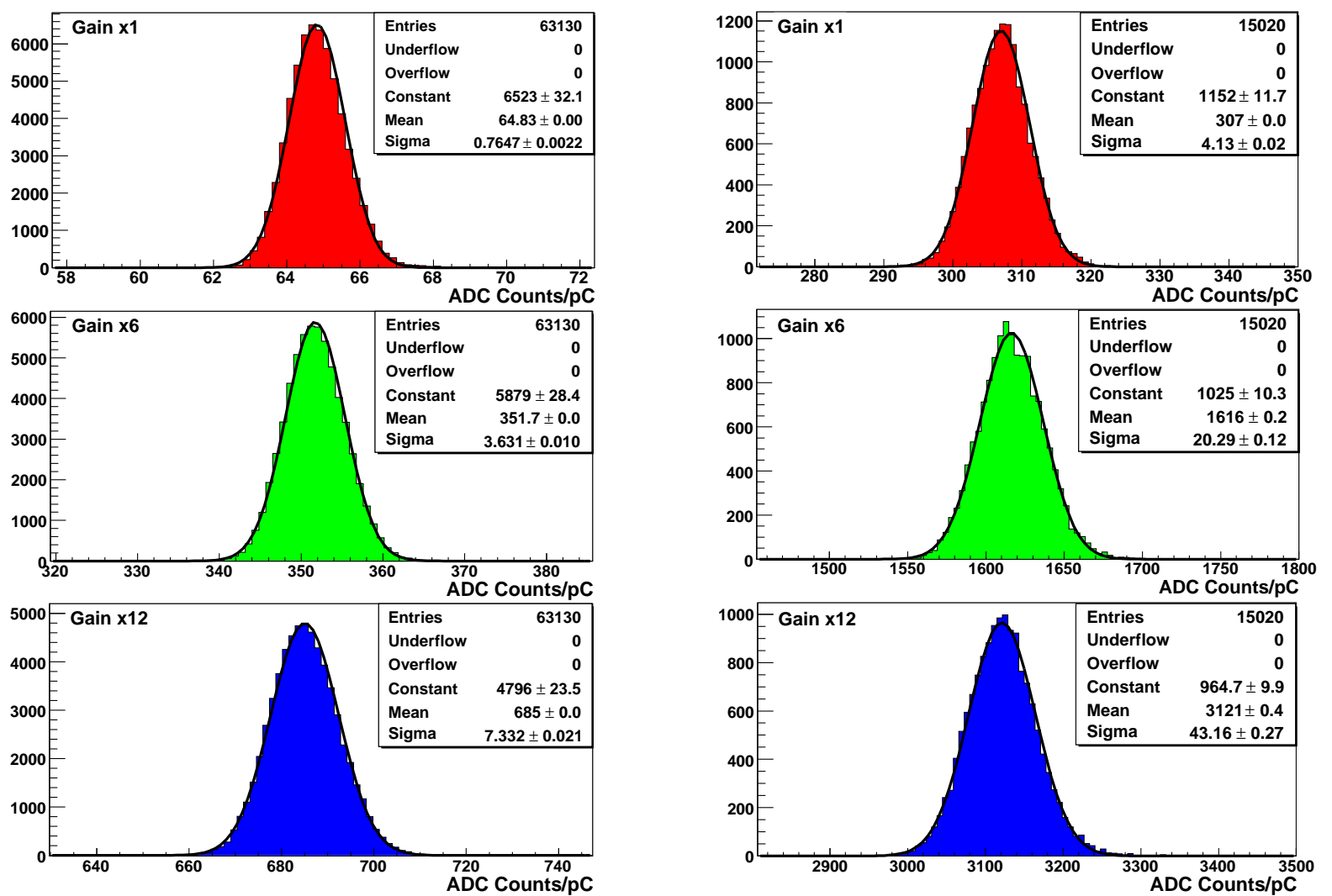

Figure 5.11: Slope distributions of the injected charges versus pulse height in ADC counts/pC for all the barrel (left) and all the endcap (right) VFE cards and the three gains 1, 6, and 12 .

\begin{tabular}{|c|c|c|c|c|}
\hline \hline & \multicolumn{2}{|c|}{ Barrel } & \multicolumn{2}{c|}{ Endcap } \\
Gain & Slope & $\sigma /$ mean (\%) & Slope & $\sigma /$ mean (\%) \\
\hline 1 & 64.83 & 1.18 & 307 & 1.35 \\
6 & 351.7 & 1.03 & 1616 & 1.26 \\
12 & 685 & 1.07 & 3121 & 1.38 \\
\hline Gain & Offset & $\sigma /$ mean (\%) & Offset & $\sigma /$ mean (\%) \\
\hline 1 & -6.15 & 14.97 & -21.49 & 11.28 \\
6 & 2.39 & 46.21 & -12.19 & 44.14 \\
12 & 1.75 & 55.99 & -7.12 & 77.69 \\
\hline \hline
\end{tabular}

Table 5.6: Mean value of the slopes (ADC count/pC) and offsets (ADC count) of the injected charges versus pulse height and its dispersions (\%) for all the barrel and endcap VFE cards for three gains 1,6 , and 12 . 

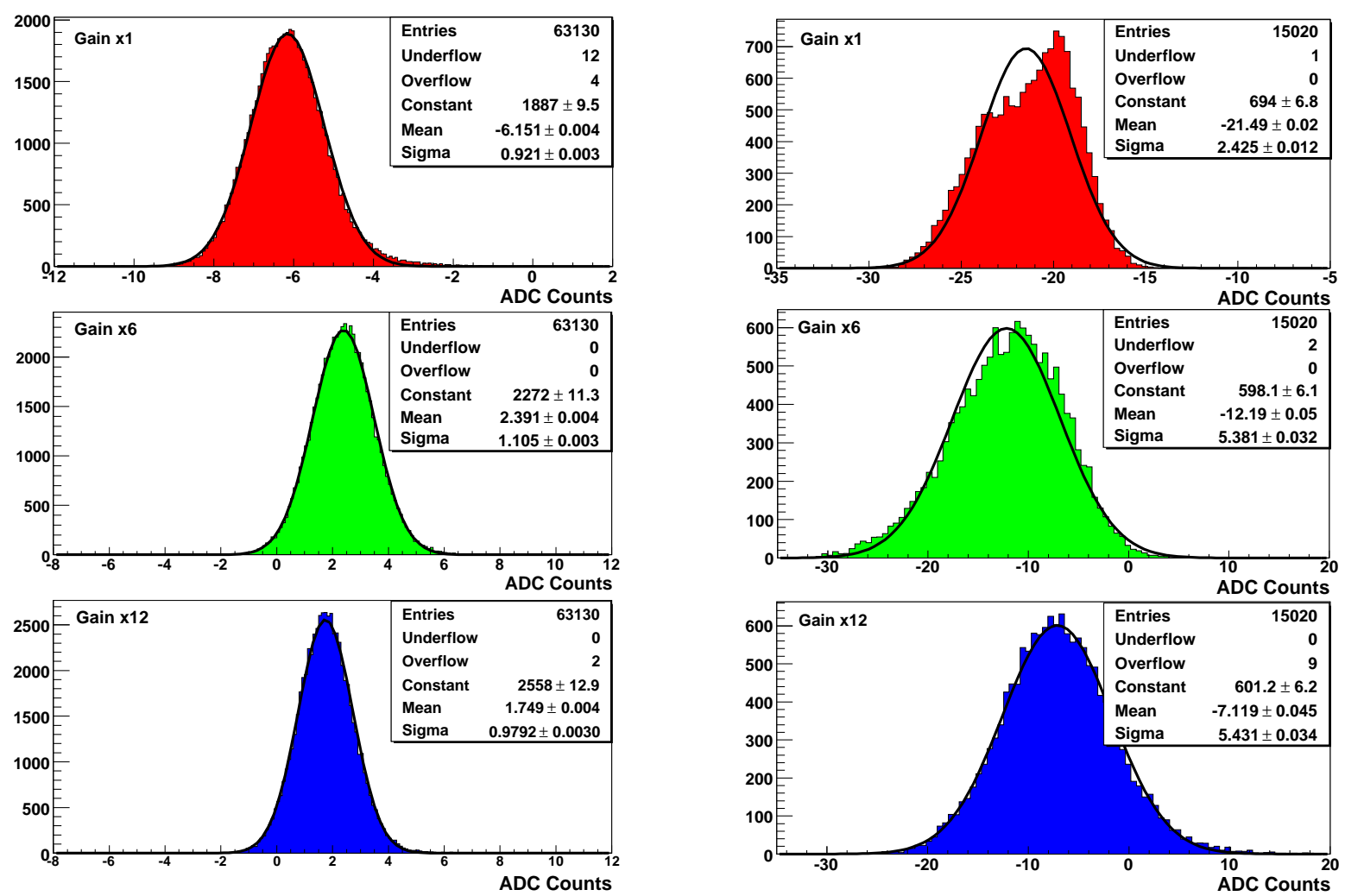

Figure 5.12: Offset distributions of the injected charges versus pulse height in ADC counts for all the barrel (left) and all the endcap (right) VFE cards and the three gains 1, 6, and 12.

\section{Intercalibration}

Since each VFE card is measured in any of the 6 different test boards, it was necessary to introduce an intercalibration among the boards in order to suppress their slight construction differences. In addition, the correction on time variations of the calibration system, which were monitored by periodic measurements of 6 reference VFE cards on the same test board during the whole calibration period, were also included in the intercalibration. A measured slope of the injected charges set for each channel and gain is normalized to the test board which was chosen as a reference. The intercalibration coefficient is then computed for each gain separately. Contributions of the differences of the test boards and time variation to the slope dispersions for gain 12 are around $20 \%$ and $10 \%$ for the barrel and endcap, respectively. Smaller improvement for the endcap VFE cards is due to the fact that the calibration system was already well tuned and understood. The values of the slope dispersion before and after intercalibration are summarized for all three gains in Tab. 5.7 and an example of the slope distributions before and after intercalibration is shown in Fig. 5.13.

\subsubsection{Gain ratios}

The laboratory gain ratio can be determined as the ratio of slopes of the calibration curve between the gains. Mean values and corresponding dispersions over all the measured channels 


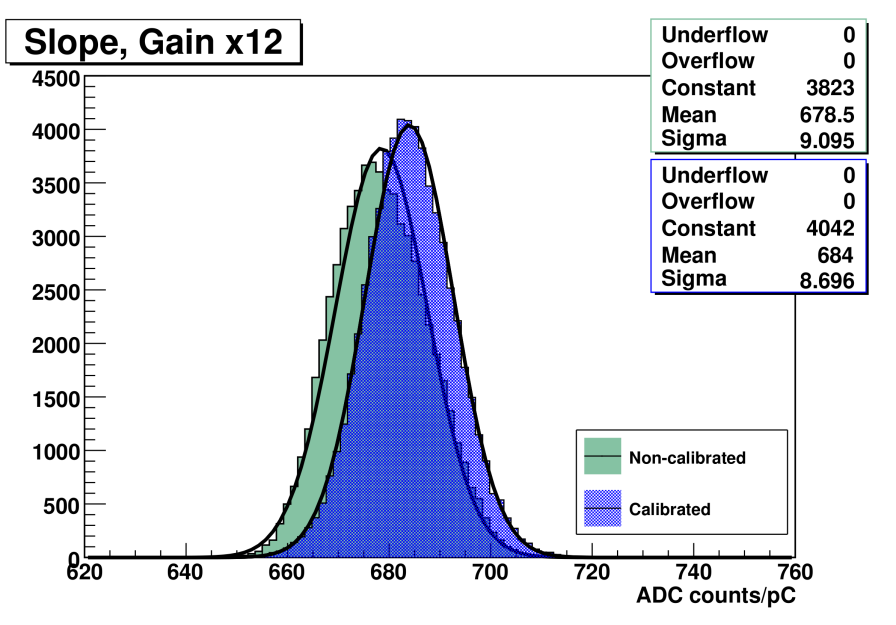

Figure 5.13: Slope distributions of the injected charges versus pulse height in ADC counts/pC for gain 12 for all the barrel VFE cards before and after the test board and time intercalibration.

\begin{tabular}{|c|c|c|c|}
\hline \hline \multirow{2}{*}{ Gain } & \multicolumn{3}{|c|}{ Barrel } \\
& $\begin{array}{c}\sigma / \text { mean (\%) } \\
\text { Non-calibrated }\end{array}$ & $\begin{array}{c}\sigma / \text { mean (\%) } \\
\text { Calibrated }\end{array}$ & Difference (\%) \\
\hline 1 & 1.35 & 1.18 & 12.59 \\
6 & 1.21 & 1.03 & 14.88 \\
12 & 1.27 & 1.07 & 18.34 \\
\hline \multirow{3}{*}{ Gain } & $\sigma /$ mean (\%) & $\sigma /$ mean (\%) & Difference (\%) \\
& Non-calibrated & Calibrated & \\
\hline 1 & 1.42 & 1.35 & 4.92 \\
6 & 1.38 & 1.26 & 8.70 \\
12 & 1.53 & 1.38 & 9.75 \\
\hline \hline
\end{tabular}

Table 5.7: Dispersions of the slopes for gain 1, 6, and 12 before and after the test board and time intercalibration. 
are summarized in Tab. 5.8 and depicted in Fig. 5.14 for the barrel as well as for the endcap VFE cards. The gain ratio show small dispersion among the channels and fully comply the specifications.
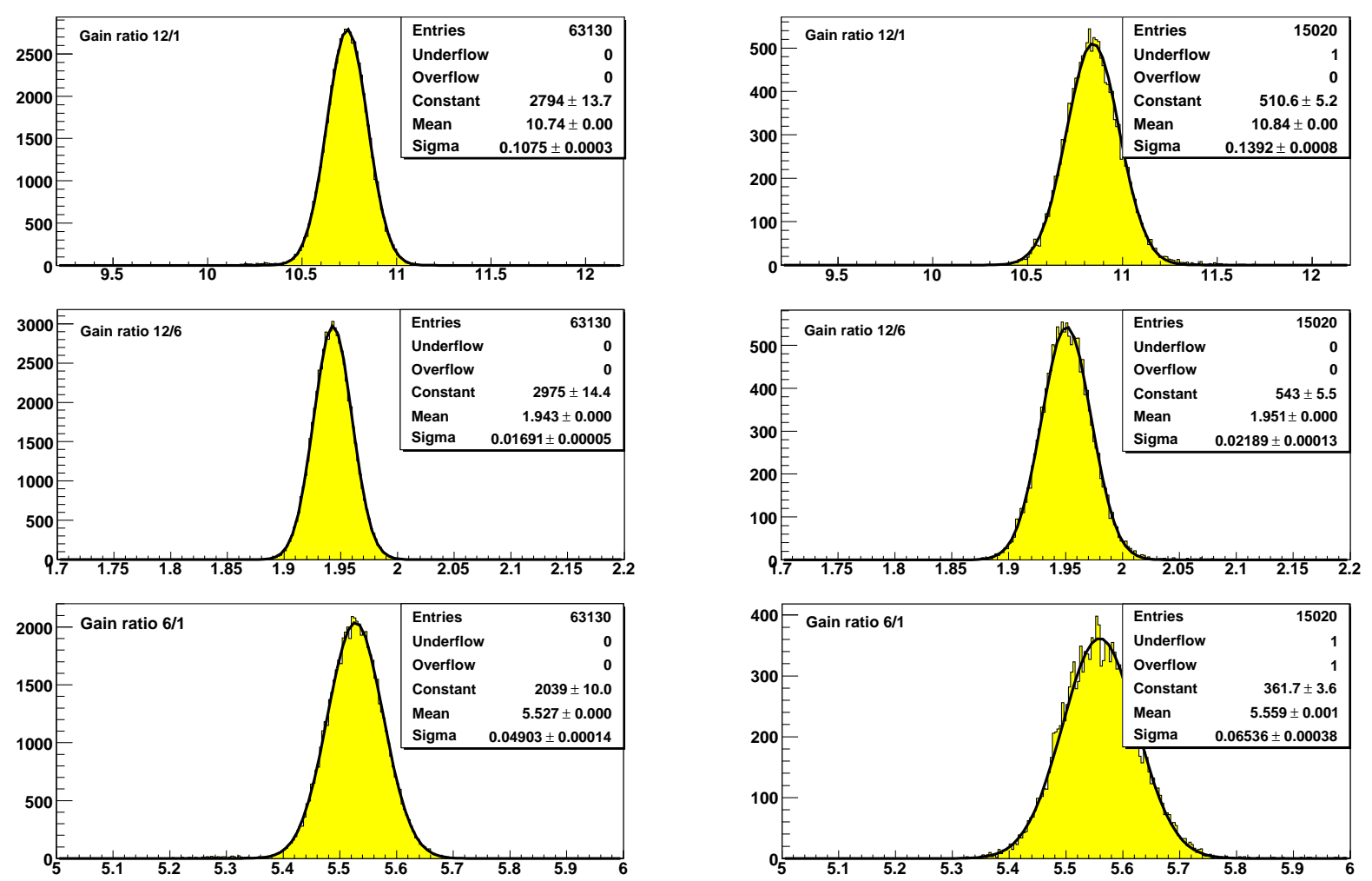

Figure 5.14: Gain ratio (12/6, 12/1, and 6/1) distributions for all the barrel (left) and endcap (right) VFE cards.

Precision of knowledge of the gain ratio plays a very important role for high or extremely high energies where switching to gains 6 or 1 occurs. For this purpose, it was necessary to develop an alternative technique for the gain ratio determination which could also be used in situ during ECAL operations. Hence, the MGPA test pulse unit was used for gain ratio computation. Through this unit it is possible to inject a test charge directly into the input of the MGPA pre-amplifier. Because the gain path can be forced to a particular choice by stopping one or more ADC channels, it is possible for the same injected charge to produce signals from different gain stages. Thus a ratio of the reconstructed signal amplitudes is equal to the gain ratio.

The gain ratio delivered by the test pulse and extracted from the slopes were compared and it was found that both methods give comparative results, well-correlated among channels, but with a small systematic shift. Fig. 5.15 shows a distribution of the relative gain 12/6 extracted from the slope of the calibration curves and measured by a test pulse of $4.1 \mathrm{pC}$ for the 340 VFE cards which are now assembled in the supermodule SM17. The correlation and relative dispersion for the same gain ratios are depicted in Fig. 5.16. The mean difference is around $1 \%$, which is due to different circuitry for the injected charge (different injected capacitors are used) and the contribution of the different main sources: the external charge used for slope 


\begin{tabular}{|c|c|c|c|c|}
\hline \hline & \multicolumn{2}{|c|}{ Barrel } & \multicolumn{2}{c|}{ Endcap } \\
Ratio & Mean & $\sigma /$ mean (\%) & Mean & $\sigma /$ mean (\%) \\
\hline $12 / 1$ & 10.74 & 1.001 & 10.84 & 1.284 \\
$12 / 6$ & 1.943 & 0.87 & 1.951 & 1.121 \\
$6 / 1$ & 5.527 & 0.887 & 5.559 & 1.175 \\
\hline \hline
\end{tabular}

Table 5.8: Mean values of the gain ratios and corresponding dispersions for all the barrel and endcap VFE cards.

determination and MGAP internal DAC in case of test pulse.

From the above mentioned results, one can conclude that the gain ratio can be precisely measured only directly on the fully equipped supermodule, i.e. during the test beam or in situ at point 5. For this reason a special study of the gain ratio in order to evaluate accurately its behaviour were performed during the test beam and discussed in detail in Chap.6.7.

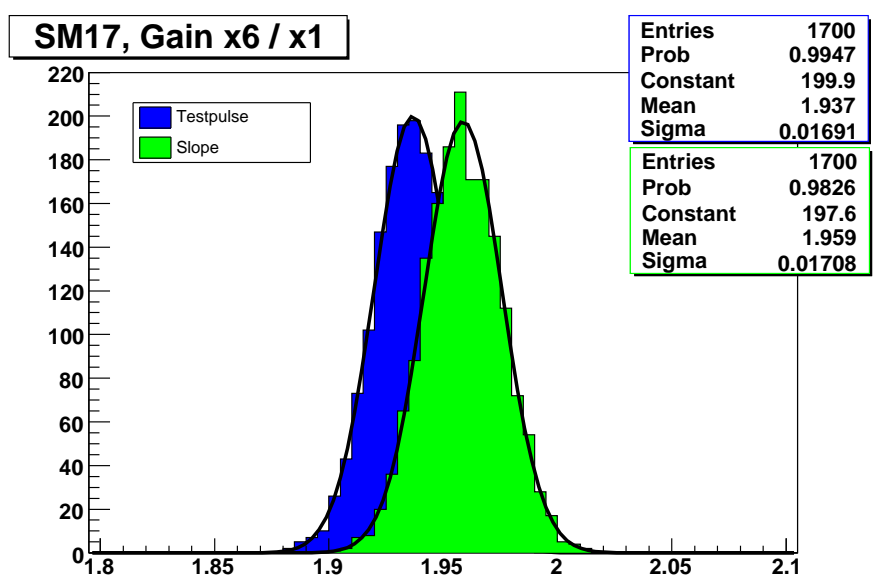

Figure 5.15: Distribution of the gain ratio 12/6 extracted from the slope (green) and measured using a test pulse charge of $4.1 \mathrm{pC}$ (blue) on the calibration bench at IPN Lyon for $340 \mathrm{VFE}$ cards (1,700 channels) which are now assembled in the supermodule SM17.

\subsubsection{Pedestal and electronic noise}

Pedestal is the electronic baseline on which an electrical signal is carried. Its value is programmable using a simple DAC circuit internal to the MGPA which is controlled externally by an $I^{2} C$ interface. Correct functionality of the pedestal setting was verified in two different but complementary ways. In the first case, special pedestal measurements for each gain were performed. The DAC value is varied from 0 up to 100 and corresponding pedestal values are recorded. Fig.5.17 shows pedestal versus DAC values for one VFE card.

After setting the pedestal, fluctuation was evaluated from dedicated pedestal runs when no charge was injected. In addition, the pedestal was also computed from pre-samples taken ahead 

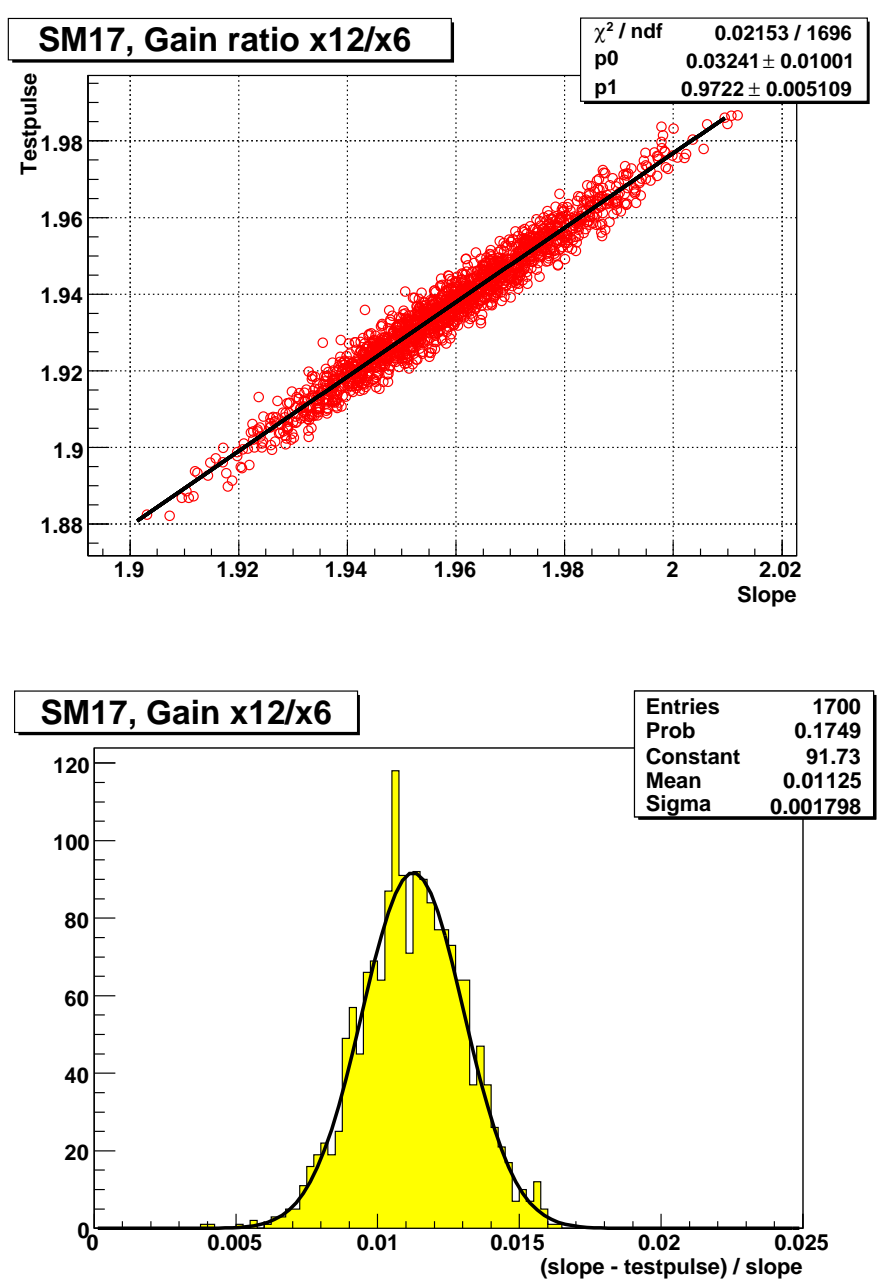

Figure 5.16: Correlation (top) and relative difference (bottom) between the gain ratio 12/6 extracted from the slope and measured by the test pulse. The gain ratio was determined on the calibration bench at IPN Lyon for 340 VFE cards (1,700 channels) which are now assembled in the supermodule SM17.

of the injected charge. These tests were performed for each gain separately.

An example of the noise distribution measured directly on the calibration bench for barrel VFE cards is displayed in Fig. 5.18. VFE cards for which the pedestal could not be set correctly or with RMS noise greater than $10 \sigma$ of the mean noise distribution for all measured channels were rejected. The noise values for the calibration bench are greater than for the ECAL due to the important contribution from calibration system itself.

In situ, electronic noise was measured during the test beam on fully assembled supermodules by applying the amplitude reconstruction weight method [10] on the pedestal runs. A typical mean value of the electronic noise for a single channel over an entire barrel supermodule $(1,700$ channels) is around $1 \mathrm{ADC}$ count $(\sim 37 \mathrm{MeV})$ for gain 12 . This value includes all contributions to the noise over the whole ECAL electronics chain. Evaluation of the electronics noise performance is given in detail in Chap. 6.6. 


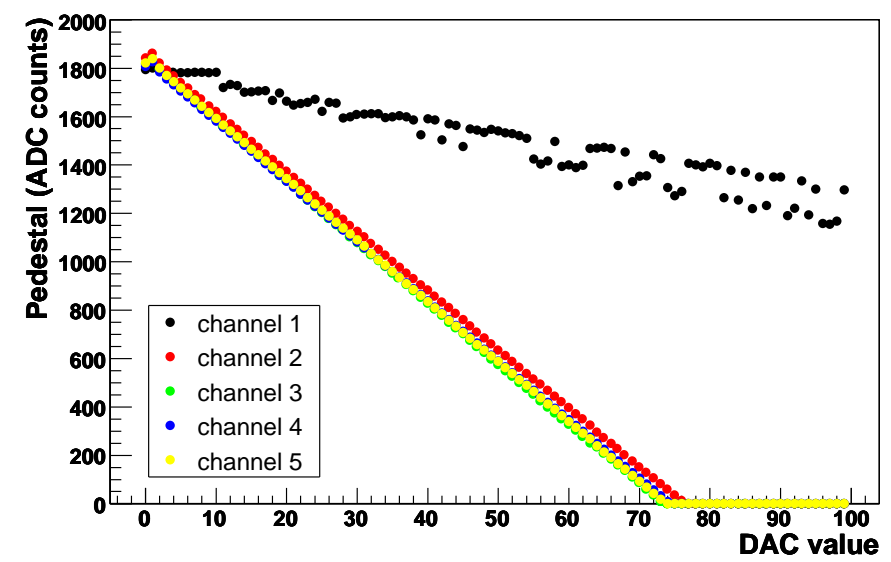

Figure 5.17: Pedestal versus DAC value for five channels of a barrel VFE card, gain 1 . The channel 1 does not work correctly, hence this VFE card has been rejected.

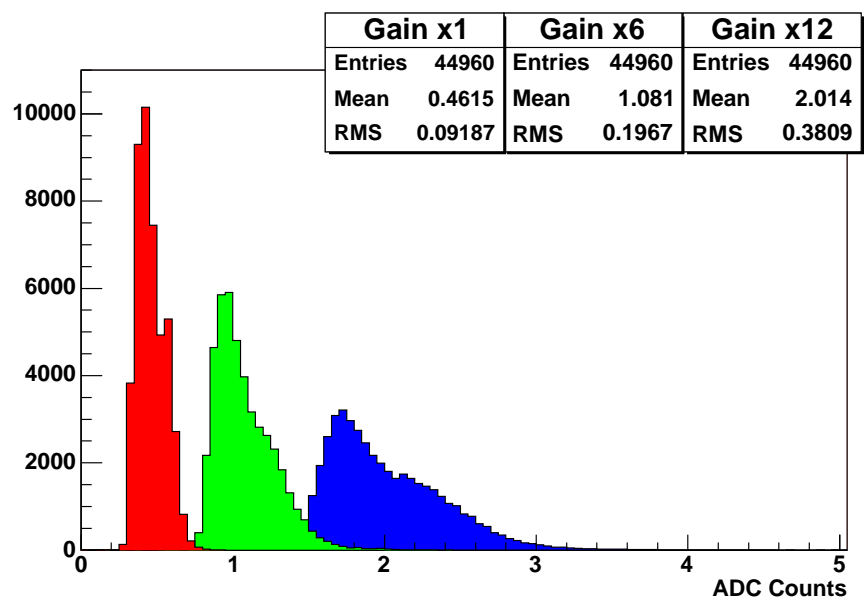

Figure 5.18: The noise distribution measured on the calibration bench for 8,992 barrel VFE cards in ADC counts for gains 1, 6, and 12. 


\subsubsection{Linearity}

The linearity of the VFE card response, i. e. the linearity of the calibration curve fitted by a straight line was verified for each channel and all three gains by the investigation of its residuals. In Fig. 5.19, where the residual values are plotted as a function of the injected charge for all three gains and all measured barrel and endcap VFE cards. The similar behaviour is for the highest and middle gain and slightly different in the lowest gain 1 especially in the case of the endcap. The non-linearity was quantified according to Eq. 4.2 and its values for all the three gains and for the barrel and endcap VFE cards are summarized in Tab. 5.9.
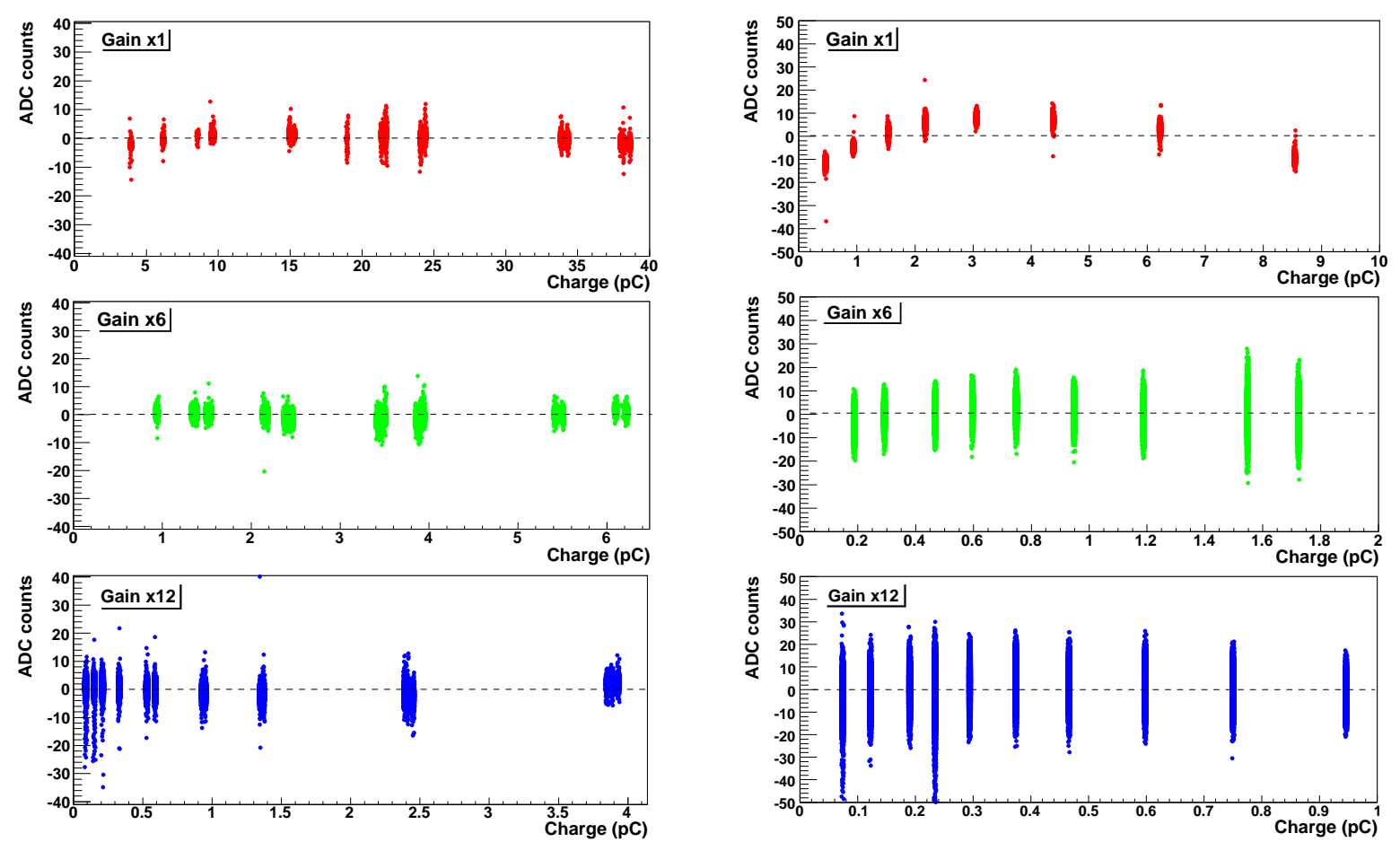

Figure 5.19: Residuals value delivered from the linear fit of the VFE response on the injected charge versus the injected charge for all the three gains for all the barrel (left) and endcap (right) VFE cards.

\begin{tabular}{|c|c|c|}
\hline \multirow[t]{2}{*}{ Gain } & \multicolumn{2}{|c|}{ Non linearity (\% fullscale) } \\
\hline & Barrel & Endcap \\
\hline 1 & 0.1 & 0.45 \\
\hline 6 & 0.1 & 0.14 \\
\hline 12 & 0.12 & 0.14 \\
\hline
\end{tabular}

Table 5.9: Non-linearity of all the barrel and endcap VFE cards measured over a full dynamic range for all three gains. 
As was shown above, the VFE cards fulfill the settled specification requirements on the linearity. Nevertheless, the study of the gain ratio measurement, which is described in detail in Chap.6.7, needs to be determined at the permille level. Such a precision of the gain ratio requires additional study of the VFE linearity to elaborate the effect of the electronics non-linearity on its measurement.

\subsubsection{Precise linearity study}

A precise linearity study cannot be performed with the limited number of injected charges that are obtained in the experimental set-up with the attenuator as it is used for the VFE calibration procedure. Therefore the configuration of the VFE calibration system was modified in the following way to obtain an experimental set-up allowing the fine scan of the VFE response. The attenuator was set to a fixed attenuation of $0 \mathrm{~dB}$ and the Agilent pulse squared generator was programed to generate series of voltage pulses in a range from 0 to $1.5 \mathrm{~V}$. So a set of injected charges from 0 to $15 \mathrm{pC}$ with $0.1 \mathrm{pC}$ steps were generated via the precise $10 \pm 0.01 \mathrm{pC}$ capacitor as in the standard configuration. A schematic drawing of the experimental set-up for the above described study of linearity is shown in Fig. 5.20.

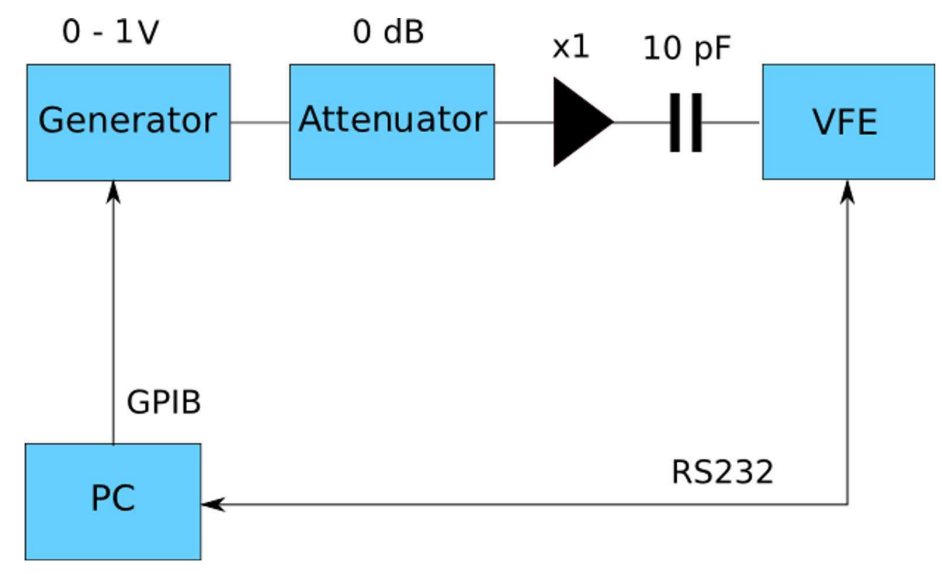

Figure 5.20: Experimental setup for the precise linearity study.

The VFE response as a function of the injected charge over the full measured range $(0-$ $15 \mathrm{pC}$ ) is shown for one VFE channel in Fig. 5.21 left. Fig. 5.21 right shows the residuals of the linear fit for each injected charge. The sudden change at $10 \mathrm{pC}$ (see Fig. 5.21 right) is due to the switching of the internal gain of the generator at $10 \mathrm{~V}$ and thus strongly affects the linearity measurement. The manufacturer of the Agilent generator guarantees its integral linearity only better than $1 \%$. On the other hand, much better linearity can be achieved with this apparatus if the measurement is performed without generator internal gain switching. In this case, the linearity measurements have to be performed only for injected charges smaller than $10 \mathrm{pC}$. In addition, an independent study of the generator linearity must be also performed.

In order to evaluate the reliability of the measurement and to avoid possible non-linearity which can come from the system (i.e. generator) and not from the card itself, the six barrel reference cards (30 channels) were measured in both configurations: with the set of charges delivered from the generator as described above and with the standard VFE calibration con- 

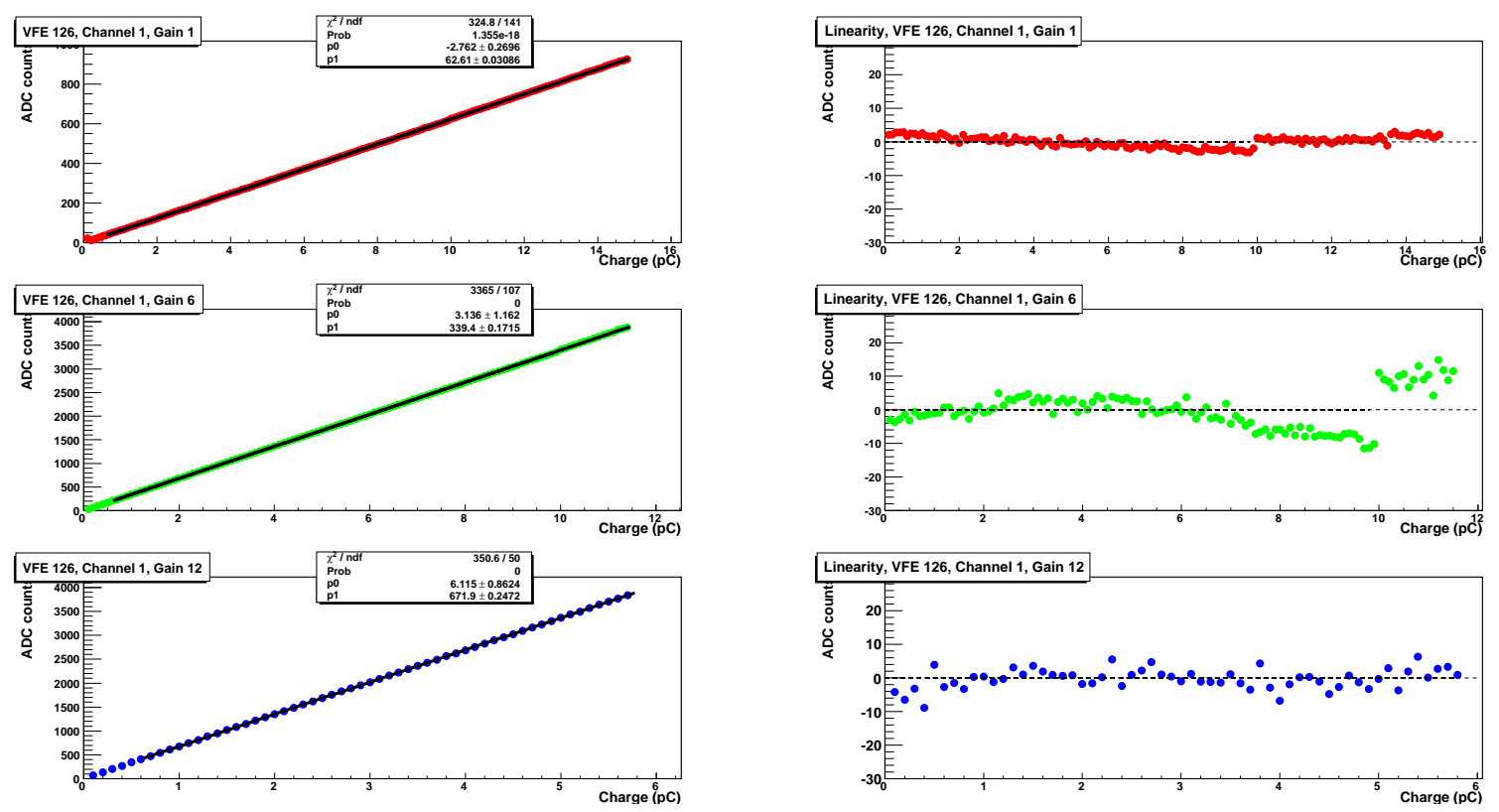

Figure 5.21: Left: VFE response as a function of the injected delivered by the pulse squared generator for all the three gains. Right: The residuals from the linear fit versus injected charge.

figuration, i.e. with the set of charges generated by the calibrated attenuator. The values of the measured charge for the same pulse height were compared and their relative differences are plotted as a function of the charge for the gain 6 in Fig. 5.22. From the figure it is clear that all the measured channels behave similarly and the average difference in the VFE response is on the permille level. Nevertheless, the small observed differences in the pulse height were also introduced in the linearity study to verify its influence on the results. Since no significant change in the VFE response was found, one can conclude that the generator is linear in the range from 0 to $10 \mathrm{pC}$. Thus, the non-linearity effects measured with this configuration come from VFE electronics and not from the measurement system.

Due to the reason described above, the charges for gains 1 and 6 were limited to $10 \mathrm{pC}$ and an upper threshold for the highest gain 12 was set at $6 \mathrm{pC}$ to avoid the saturation as is shown in Fig. 5.23 left. Thus, the dynamic range of gain 6 (up to $12 \mathrm{pC}$ ) is almost completely covered while the low gain 1 is covered only partly and therefore cannot be included in this linearity study. For this reason linearity was investigated only for gains 12 and 6 and the results are displayed in Fig. 5.23 right. From the plot, one can conclude that gain 12 can be assumed as linear over its whole dynamic range. On the other hand, the effect of non-linearity in gain 6 is clearly shown. Nevertheless, the effect is small (the full scale non-linearity is about $0.3 \%$ in gain 6), but will lead to the underestimation of the measured gain ratios, which have to be know with an accuracy of few permille (see Chap.6.7). 

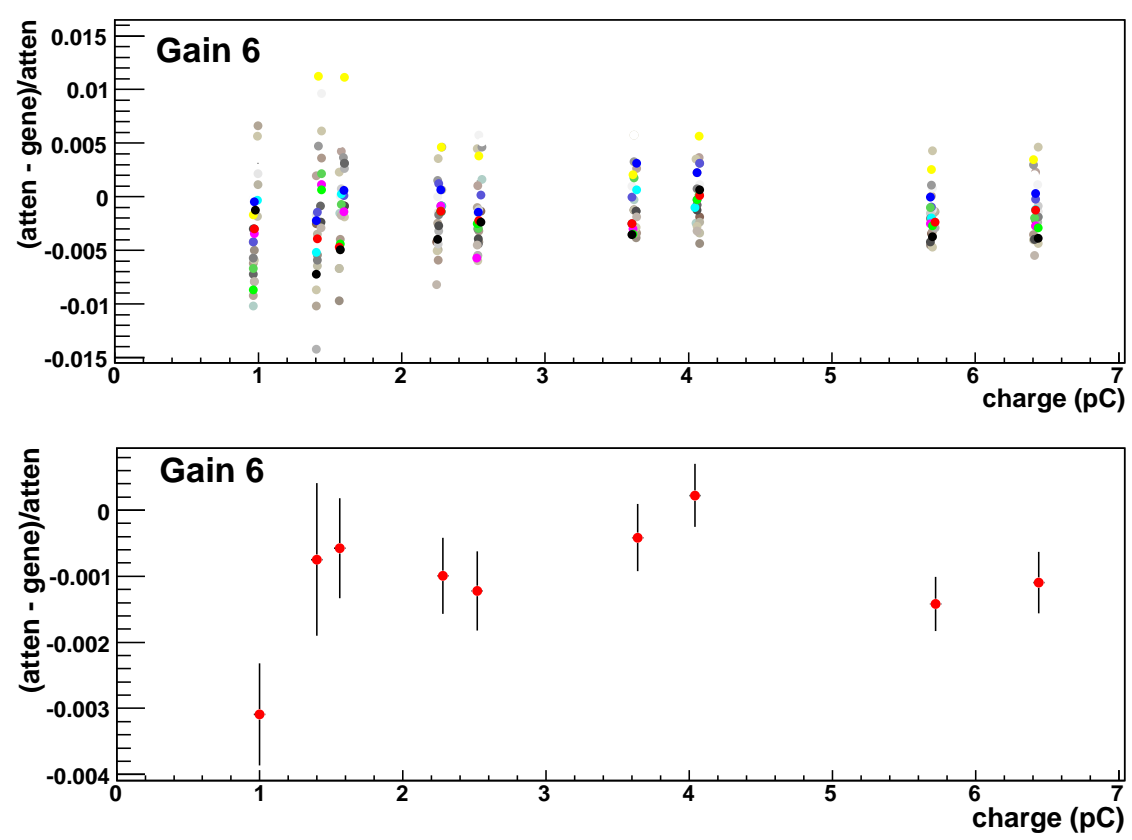

Figure 5.22: Relative difference between pulse height (as a response of the injected charge delivered from the generator and by help of the attenuator) as a function of the charge for 30 measured channels (top) and for the mean over these channels (bottom).
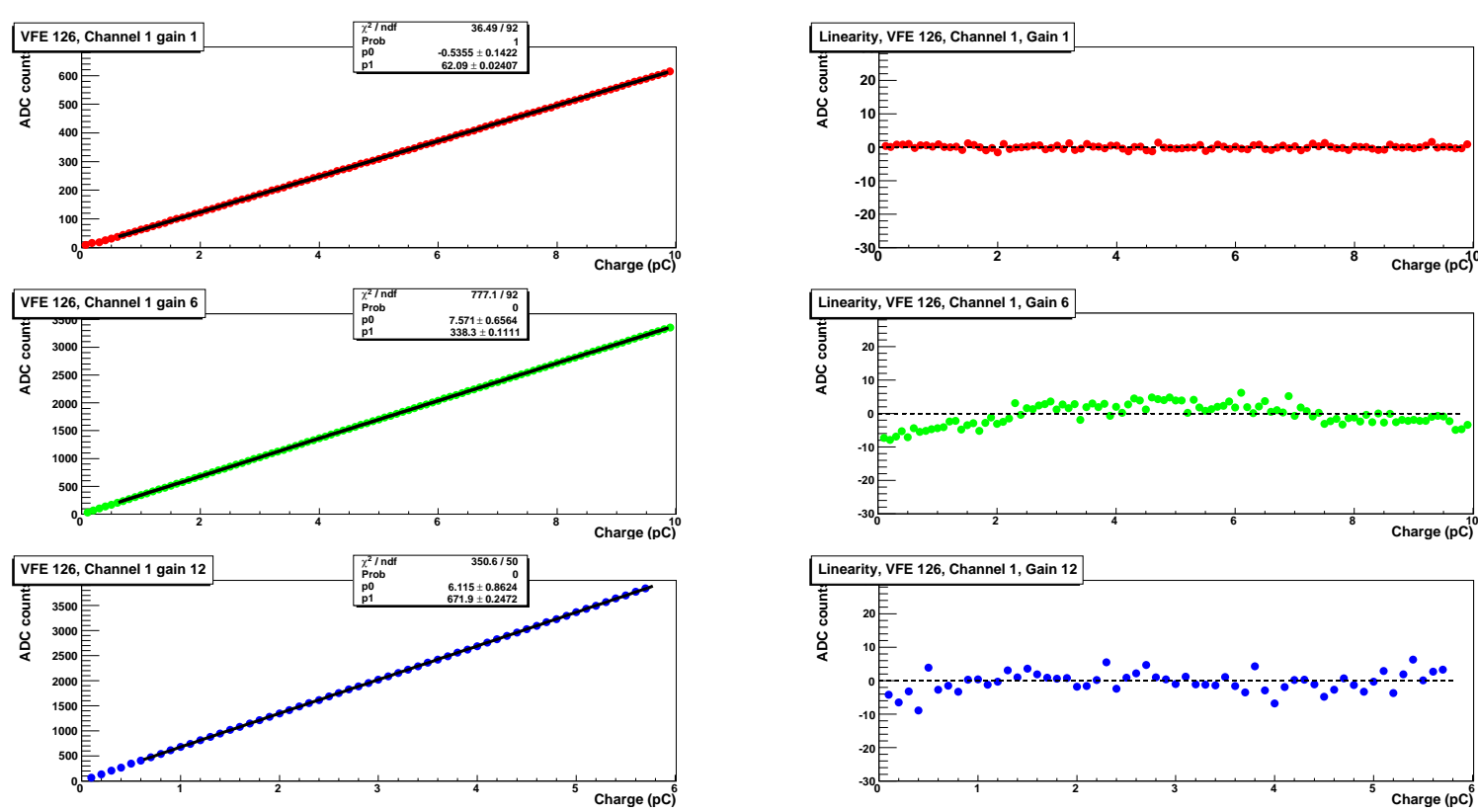

Figure 5.23: Left: VFE response as a function of the injected delivered by the pulse squared generator for all the three gains. Right: The residuals from the linear fit versus injected charge. 


\subsubsection{Electronic saturation}

The saturation of the electronic occurs at about $1.5 \mathrm{TeV}$ and $3 \mathrm{TeV}$ in the barrel and endcap. Nevertheless, some physics processes (beyond the Standard Model) can exceed these energies in a crystal and hence the dynamic range of gain 1 . If this happens, the information in a saturated channel is unuseful and the energy is estimated by help of adjacent crystals. For that reason, it is necessary to investigate the behaviour of the VFE channels in case of its saturation.

Since the highest and middle gains in case of saturation are not used, the saturation effect was studied only in gain 1 using the six barrel reference cards. Since the dynamic range of a barrel VFE card ends around $60 \mathrm{pC}$, a set of 22 different injected charges was chosen to cover a range between 53 and $66 \mathrm{pC}$, where the upper limit is well above the specification and corresponds to $>2 \mathrm{TeV}$ energy.
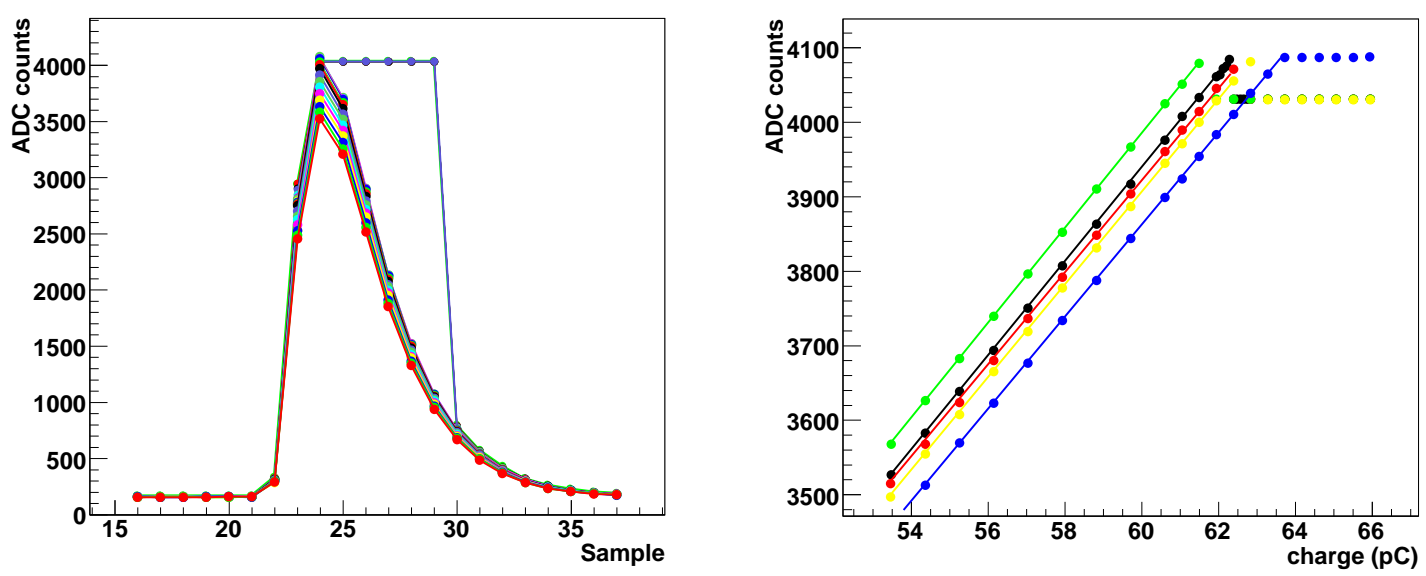

Figure 5.24: ADC output for different injected charge in a single channel (left) and linearity plot for five channels of one barrel VFE board (right).

In Fig. 5.24 left, the behaviour of the ADC is shown. The hysteresis mode (see Chap. 4.4.2) leads to the return of five consecutive samples with the same gain, so five saturated samples. The saturation value can be different from channel to channel, but is always higher than 3900 ADC counts and is characteristic for a given channel. This effect is clearly seen for one VFE card in Fig. 5.24 right, where the VFE response is plotted as the function of the injected charge. In this plot, the points determined as the maximum of the signal pulse shape fitted be the analytic function are defined in Eq. 5.2.

As we do not measure any non-linearity before saturation, it can be concluded, that for all the studied channels, the ADC saturates before the MGPA and that once the saturation is reached, the ADC value does not change with increasing injected charge. Different channels saturate for different charges, but always higher than $61 \mathrm{pC}$. The MGPA appears to be linear, within a few ADC counts, up to the saturation point.

It was also proved that saturation does not affect the timing corresponding to the maximum of the signal collection needed for L1 triggering and thus does not distort the LHC bunch crossing identification [11]. 


\subsubsection{Temperature dependence measurement}

A study of the temperature dependence was carried out during the preparation phase for the VFE calibration to evaluate the influence of the environmental condition on the performance of the VFE card. Before the beginning of the calibration program it was foreseen that the VFE cards would be calibrated at four regional centers where the temperature in the laboratory can strongly vary within several degrees due to its localisation as well as during year.

To evaluate the influence of the temperature fluctuations on the calibration, a precise temperature dependence measurement was performed. The experimental area was equipped with a powerful air-conditioning system that allows to vary the room temperature in a range of $\sim 14^{\circ} \mathrm{C}$ $\left(13.25-27.5^{\circ} \mathrm{C}\right)$. The 6 barrel reference VFE cards were repeatedly measured as during the standard calibration procedure for each $0.5{ }^{\circ} \mathrm{C}$ stabilized temperature of the thermal interval.
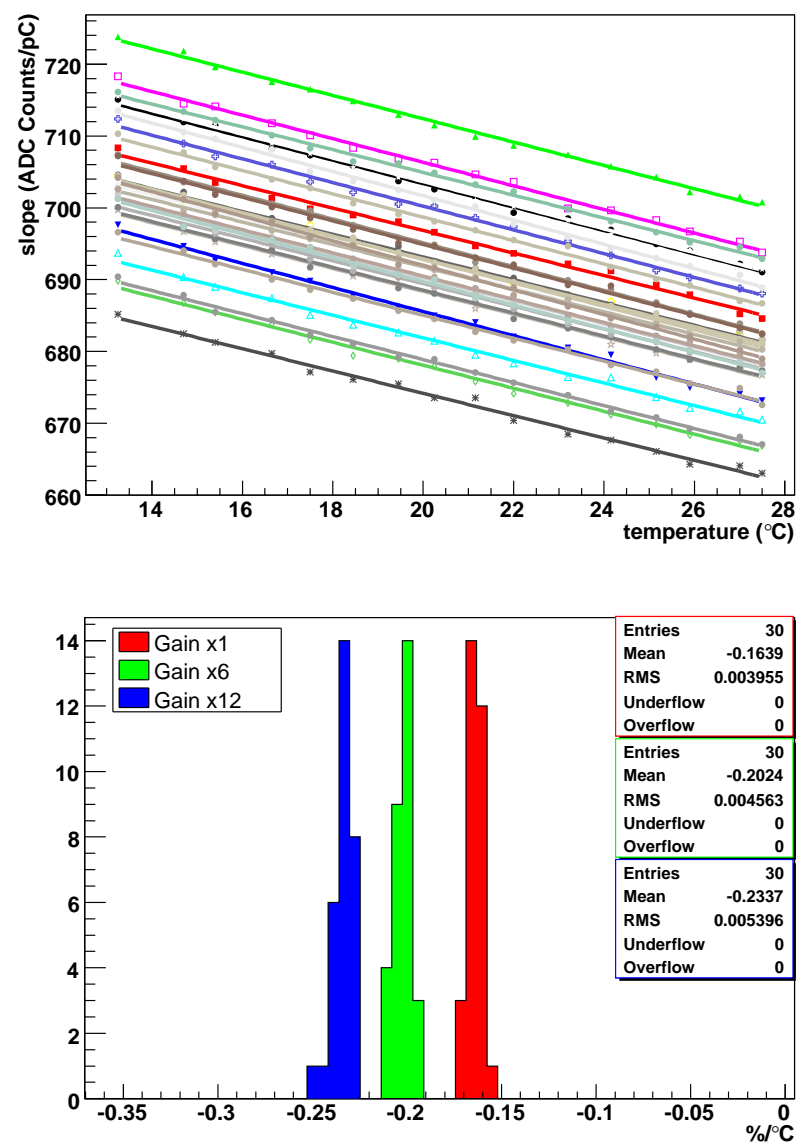

Figure 5.25: Top: The slope as a function of temperature in gain 12. Bottom: Coefficient of the temperature dependence for all three gains. The measurement performed on 6 reference VFE cards (30 channels).

It was observed, that in the measured thermal interval, the pulse height and slope values decrease linearly with increasing temperature. Coefficients of the temperature sensitivity depend on the gain and they are $-0.16,-0.2$, and $-0.23 \% /{ }^{\circ} \mathrm{C}$ for gain 1,6 , and 12 , respectively. The results 
are depicted for all the measured channels in Fig. 5.25. In order to minimize this dependence, the calibration measurement was performed as close as $18^{\circ} \mathrm{C}$ which is the working temperature of the ECAL. Therefore, each VFE card were thermalized for several hours prior to its calibration and all the measurements were performed in a temperature stabilized room $\left(18 \pm 0.3^{\circ} \mathrm{C}\right)$ that was continuously monitored. In situ, the ECAL cooling system also provides a precise stable temperature.

\subsection{Conclusion}

The individual steps of the VFE card QA programme and its main results have been presented in this chapter. The test program for all the 12,240 barrel and the $\sim 3,000$ endcap VFE boards has been completed. The dispersion in the gains is found to be about $1.2 \%$ for the barrel and $1.4 \%$ for the endcap, which well fulfill the specification (4\%). Noise, linearity and gain ratios are also complying with the CMS detector criteria. Only around $2 \%$ of them failed the tests and have been rejected, the rest have been assembled into barrel supermodules and endcap supercrystals. The results are registered in a database and can be used for a first intercalibration of the ECAL. The results obtained during the QA program were verified in summer 2006 and 2007, when several fully equipped supermodules and supercrystals were tested and calibrated with high energy electrons during the test beam campaign that is described in the following chapter. 



\section{Bibliography}

[1] C. Biino et al., "The CMS ECAL very front end electronics production and test," in $10^{\text {th }}$ Workshop on Electronics for LHC Experiments LECC 2004, Boston, USA, Sep 2004, pp. 107-111.

[2] J. Blaha et al., "Performance of CMS ECAL very front end electronics," in 12th Workshop on Electronics for LHC Experiments LECC 2006, Valencia, Spain, Sep 2006, pp. 174-178.

[3] "Power on test for the VFE electronics," http://wwweth.cern.ch/integration/PowerOnTest/.

[4] Burn-in - An Engineering approach to the design and analysis of burn-in procedures, ser. A Wiley - Interscience publication. John Wiley \& Sons, 1990, no. ISBN 0471102156.

[5] J. Blaha et al., "Calibration of the very-front-end electronics for the electromagnetic calorimeter of the CMS experiment," Czechoslovak Journal of Physics, vol. 56, no. 8, pp. 879-881, Aug 2006.

[6] — "Calibration and performance test of the very-front-end electronics for the CMS electromagnetic calorimeter," Nuclear Physics B - Proceedings Supplements, vol. 172, pp. 168-170, October 2007.

[7] — "Calibration and performance tests of the very-front-end electronics for the CMS electromagnetic calorimeter," CERN, Geneva, Tech. Rep. CMS-CR-2007-065. CERN-CMSCR-2007-065, Oct 2007.

[8] C. Seez, "Physics requirements for electronics calibration," Talks presented at VFE Calibration Meeting, http://indico.cern.ch/contributionDisplay.py?contribId=s1t0\&confId=a045625, Dec 2004.

[9] P. Paganini and I. Van Vulpen, "Pulse amplitude reconstruction in the CMS ECAL using the weights method," CERN, Geneva, Tech. Rep. CMS-NOTE-2004-025. CERN-CMS-NOTE2004-025, Oct 2004.

[10] CMS ECAL Group, "Reconstruction of the signal amplitude of the CMS electromagnetic calorimeter," Eur. Phys. J. C, vol. 46 S1, pp. 23-35, 2006.

[11] J. Blaha et al., "Effect of ECAL electronics saturation on L1 trigger and reconstruction," CERN, Geneva, Tech. Rep. CMS IN-2007/056, Oct 2007. 



\section{Chapter 6}

\section{ECAL test beam performance}

\subsection{Introduction}

During the past several years the functional properties of the individual parts of the electromagnetic calorimeter were tested in various high energy particle beams (electrons, muons and pions). Results of the analysis of the test experiments have served to elaborate the general engineering-technical solutions of the electromagnetic calorimeter construction and optimize its spectrometric and operating properties.

The first performance tests of a large-scale system were carried out in the summers 2002 and 2003 when several parts of the barrel system were tested in the H4 test beam facility at CERN. In 2002, a submodule (M0') consisting of 400 crystals, partially equipped with the previous version of the electronics ${ }^{1}$ with 100 active channels. During the year 2003 the first supermodule SM0, containing 1700 crystals with 100 crystals read out channels with FPPA, and the second supermodule SM1 with 50 read out channels equipped with a prototype of the new on-detector electronics with the MGPA chip, were detailly investigated [2]. In the autumn of 2004 the first fully equipped barrel supermodule SM10 with almost final technical solutions underwent beam tests in high energy electrons, muon and pion beams to check reliability of the Data Acquisition (DAQ), the Detector Control System (DCS) and evaluation of the detector performance and the precalibration strategy. These tests were especially important because of the SPS maintenance shutdown scheduled for the following year, it was the last opportunity of having the system in data taking conditions with particle beams before the beginning of the barrel commissioning.

During the summer and fall of 2006, 9 out of total 36 barrel supermodules undergone systematic beam tests and were precalibrated with high energy electron beams before their installation in the experiment at Point 5. The head objectives of these tests were the supermodule precalibration, system validation, special studies and its comparison with simulation data. The main aim of the precalibration is to obtain calibration constants for each channel of all the supermodules that have been exposed to $120 \mathrm{GeV}$ electrons beam. In addition, one supermodule has been re-calibrated in order to quantify the reproducibility of the procedure.

\footnotetext{
${ }^{1}$ A signal coming from photodetectors was amplified by a Floating Point Pre-amplifier (FPPA) [1] instead a MGPA that is currently used in CMS ECAL.
} 


\subsection{Test beam objectives}

The major objectives of the test beam are summarized below. The main results concerning the first mentioned item, where an important contribution have been done as a part of this work, are presented in the following sections.

- Amplitude reconstruction procedure which includes timing and phase measurements with asynchronous and synchronous running, gain ratio measurements intended to find a reliable way of its determination that give the best energy resolution, and study of the electronics noise (spatial and time correlated noise).

- Intercalibration of nine supermodules in a electron beams of 90 and $120 \mathrm{GeV}$ and its comparison with laboratory and cosmics intercalibration.

- Systems validation, e.g. cooling systems and its control, understanding of the laser monitoring system and its long term stability, low and high voltage systems, data acquisition system (DAQ), data quality monitoring (DQM) software, and preparation for off-line data analysis.

- Energy resolution and linearity studies over a wide range of energies $(15-250 \mathrm{GeV})$, uniformity of response and energy containment correction study and its comparison with MC simulations.

- Irradiation study (usually at $90 \mathrm{GeV}$ ) - measurements of the crystal behavior under radiation when subjected to LHC-like environmental condition and comparison of the crystals' response to laser light and beam particles.

- Test of the new CMSSW software that is going to be used at LHC. The same software environment is used for reconstruction as well as for simulation.

- Combined test of the ECAL supermodule with a part of hadron calorimeter. This test has been performed in the neighbouring H2 CERN beam line area.

\subsection{H4 test beam line}

The ECAL test beam experiments use an $\mathrm{H} 4$ beam $\operatorname{line}^{2}[3]$. which is an extraction line of the Super Proton Synchotron (SPS) accelerator providing secondary or tertiary particle beams of hadrons, electrons, and muons of energies between 10 and $360 \mathrm{GeV} / \mathrm{c}$. The beam line can also be used for transport of primary protons with a maximum energy of $400 \mathrm{GeV} / \mathrm{c}$ and a typical intensities of $10^{12}$ protons per burst. The unique feature of the $\mathrm{H} 4$ beam line is the possibility to obtain a pure high resolution electron beams in a wide range of energies which are required for ECAL test beam studies.

To obtain desired particle beams with narrow momentum bites and high rates for required energies, several different configurations of the $\mathrm{H} 4$ beam line setting were used during the test beam campaigns. The first one (see Fig. 6.1 top) allows to use all types of secondary particles (electrons, pions, protons, muons, kaons, as well as low energy hadrons obtained from decays of kaons and lambdas) produced after interaction of the primary protons on the beryllium target

\footnotetext{
${ }^{2}$ The CMS H4 area is situated at the SPS North Area in building no. 887, CERN - Prevessin, France.
} 

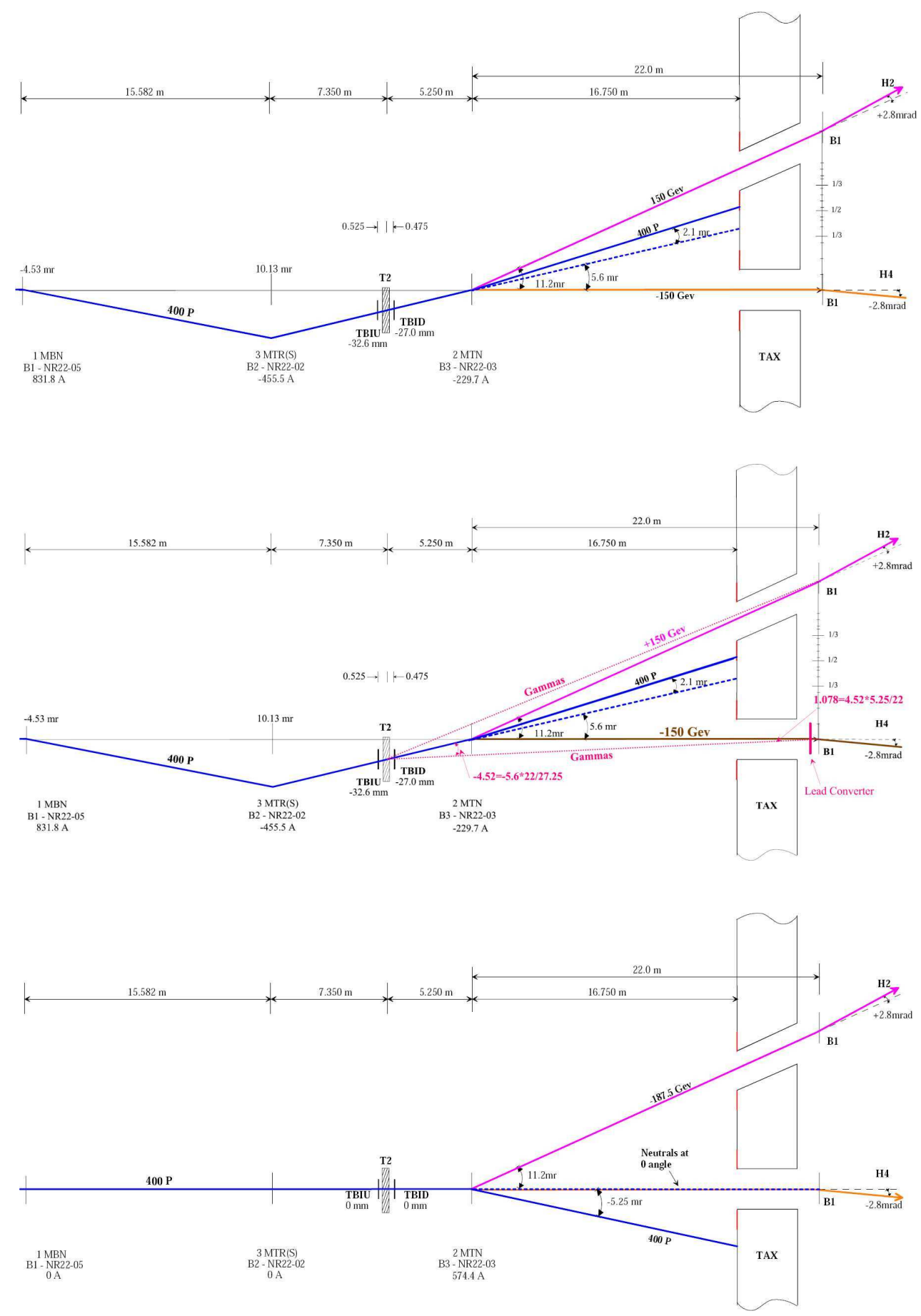

Figure 6.1: Three different settings of the $\mathrm{H} 4$ beam line configuration at CERN which were used during ECAL beam campaigns [3]. 
T2. The produced particles are separate according to their charge by the bend magnet B3, thus the particles with a negative charge are directed towards the $\mathrm{H} 4$ extraction line and particle with a positive charge to the $\mathrm{H} 2$ line. The sign of the beam can also be changed simply by inverting the polarity of the B3 magnet. The type of the secondary beam is chosen according to the particle production angle and by help of other beam optics elements along the line.

The second beam line configuration (see Fig. 6.1 middle) is similar to the first one, but especially intended for the production of pure electron beams. The neutral pions produced on the target T2 decay into photons which are then converted into electron-positron pairs by the lead converter placed between the mobile dump attenuator Target Attenuator eXperimental areas (TAX) and bend magnet B1 of the H4 extraction line. Obtained electron beams are well defined and therefore this beam configuration was used as the major one throughout the test beam campaign and particularly for intercalibration at the energy of $120 \mathrm{GeV}$. On the other hand, the configuration, which uses tertiary particles and an impinging angle to the primary particles, does not provide high rate electrons beams at higher energies.

For this reason, for test beam studies which require high statistic runs at an electron energy higher than $\sim 170 \mathrm{GeV}$, the third configuration using secondary electrons generated on the primary beam is available. Typical for this option (see Fig. 6.1 bottom) is a zero production angle for electrons which gives high electron fluxes to $\mathrm{H} 4$ and thus also provides electron beams at high energies $280-300 \mathrm{GeV}$ with a rate from several hundreds up to a thousand electrons per spill. The disadvantage of this configuration is not being able to also have high electron beams at H2. But, at the same time, pions or muons can be used alternatively.

The above mentioned configurations provide electrons beams with a fixed momentum between 20 to $300 \mathrm{GeV} / \mathrm{c}$. The intensity and momentum bite is determined by slits of collimators $\mathrm{C} 3$ and $\mathrm{C} 8$. The bending magnets (B6 and B7) which are placed between them serve as a spectrometer to measure the momentum defined by the collimator C3. An intristic and maximum momentum dispersion is $0.05 \%$ and $1.4 \%$. For energies range in ECAL test beam the momentum spread thus vary between 0.02 to $0.24 \%$. Other contributions to the beam momentum resolution came from a bremsstrahlung due to material in the beam and for higher energy $(\sim 100 \mathrm{GeV} / \mathrm{c})$ the contribution of the synchrotron radiation.

\subsection{Test beam set-up}

Each supermodule that underwent the test beam studies was fully equipped with the final design of the front-end electronics, the High Voltage (HV) and Low Voltage (LV) distributions systems, the cooling systems, and the laser monitoring system.

After the initial system test during the cosmic intercalibration, each supermodule was mounted on a calibration table installed just in front of the $\mathrm{H} 4$ beam telescope output. The table (see Fig. 6.2), remotely controlled from an operation room situated outside of the radiation zone, can rotate in both $\eta$ and $\phi$ directions and thus allows the beam to be directed towards of the any selected crystals. The crystals in each supermodule are numbered from 1 to 1700 corresponding to their arrangement in an array with 20 vertical rows (covering $\phi$ form $0^{\circ}$ to $20^{\circ}$ ) and 85 horizontal columns ( $\eta$ from 0 to 1.479). On an exposed crystal, the particles were incident at an angle of $\sim 3^{\circ}$ with respect to the direction of the crystal axis in both transverse directions, reproducing the average incident angle particles emerging from the collision region as it will be in the CMS.

As a trigger for the DAQ system, the system of six trigger scintillating counters ( $S 1$ to $S 6$, where the trigger $S 1$ is the farthermost and the $S 6$ is the closest one with respect to the 


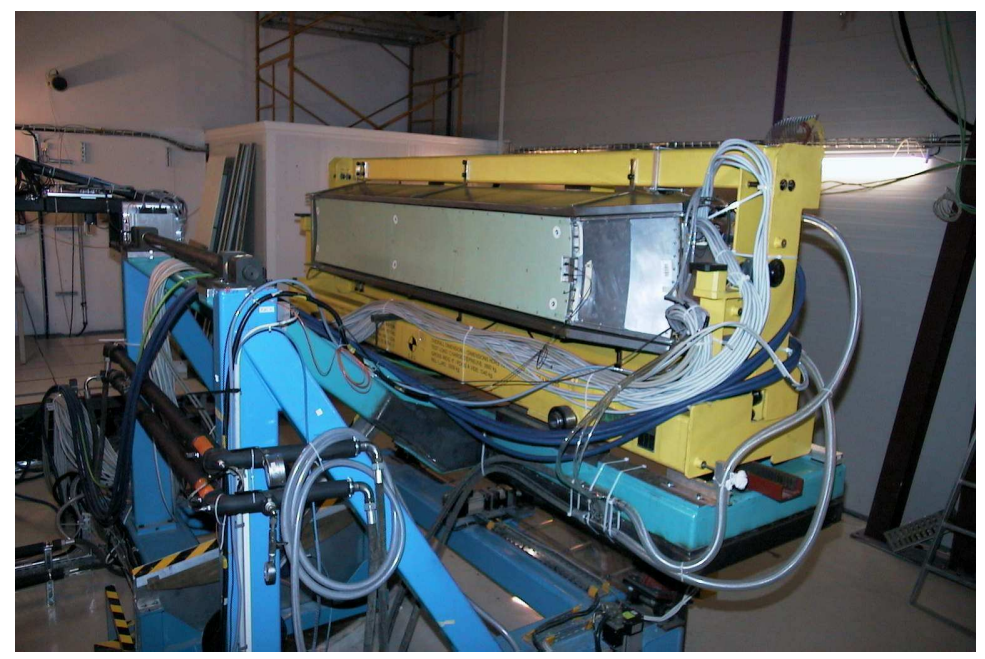

Figure 6.2: A supermodule mounted on the remotely controlled movable table allowing to direct the high energy electrons beams to the chosen crystal. The table was installed at H4 SPS beam area at CERN.

table) are placed along the beam line. In the standard configuration, the triggers select events corresponding to an area of $20 \times 20 \mathrm{~mm}^{2}$, which practically covers the whole front-face of a crystal. The number of events in each spill can be changed by setting the collimators.

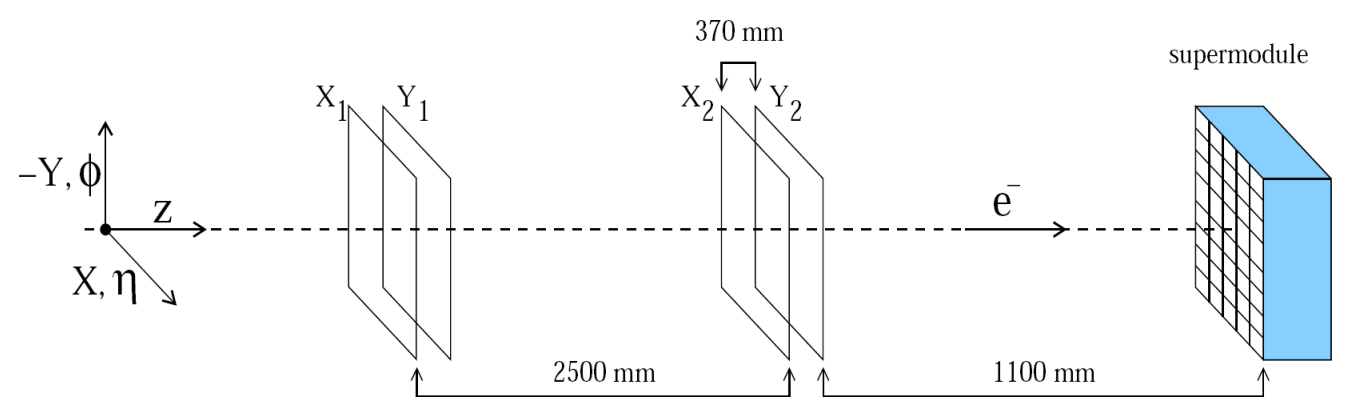

Figure 6.3: A schematic view of the position of the hodoscope planes within the experimental set-up in the $\mathrm{H} 4$ beam line at CERN.

To measure the transverse beam position and thus to precisely determine the position of the incident particle on the front-face of the crystal, a scintillating fiber hodoscope system was installed between the beam line output and the supermodule. The system consists of four measurement transversal planes (each composed from two layers of $1 \mathrm{~mm}$ semi-overlapping scintillating fibers), which were placed in two stations at a distance of $2.5 \mathrm{~m}$ along the beam axis as is seen in Fig. 6.3 [4]. Each hodoscope station provides the measurement of the beam position in both $x$ and $y$ coordinates with a precision of $\sim 145 \mu \mathrm{m}$, which allows to obtain an impact point resolution on the crystal of $250 \mu \mathrm{m}$ [4]. The hodoscope planes are oriented such, 
that $x$ and $y$ coordinates correspond to the $\eta$ and $\phi$ directions. Typical beam intensity profiles (reconstructed true impact point on the front-face of the crystal) for both coordinates are shown in Fig. 6.4 for an electron beam of $120 \mathrm{GeV}$. The shape of the profile distributions reflects the strip structure in the hodoscope construction and the difference between $x$ and $y$ directions clearly shows non-uniformity of the beam profile.
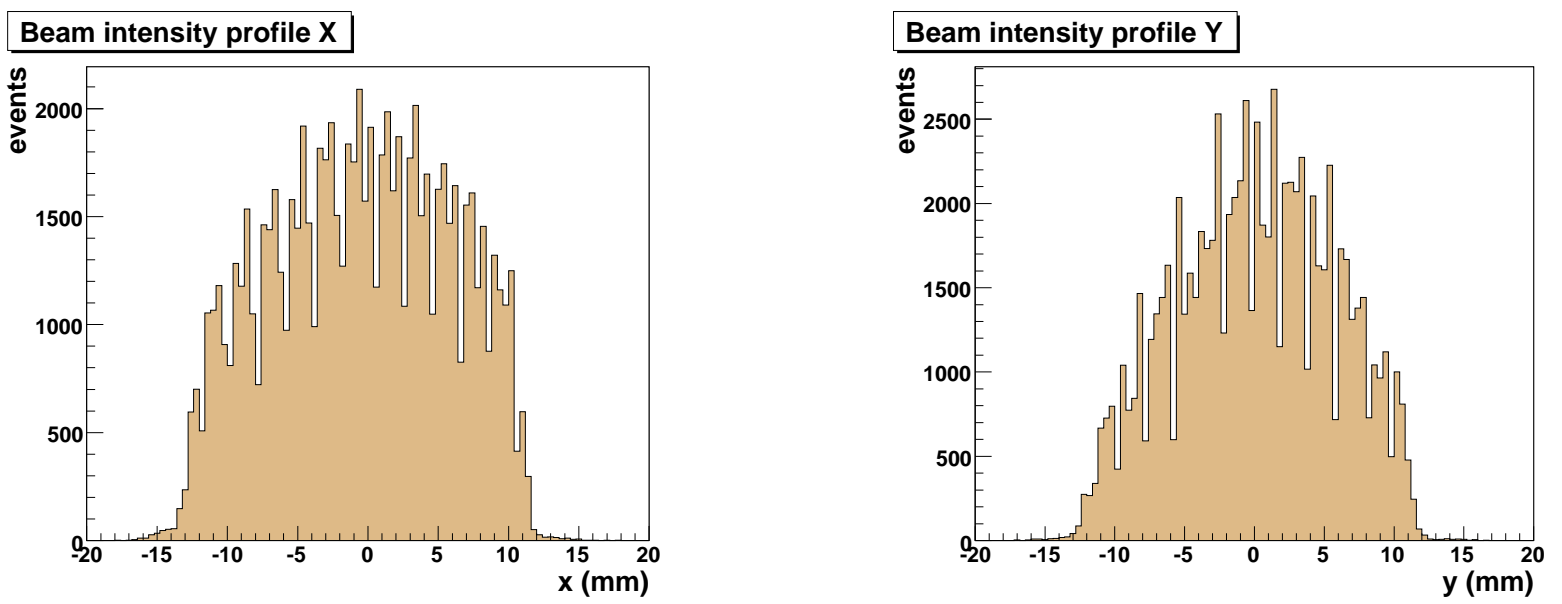

Figure 6.4: Beam intensity profiles along $x$ (left) and $y$ (right) directions measured by the scintillating fiber hodoscope for an electron beam of $120 \mathrm{GeV}$.

It must be noted that the main difference of the $\mathrm{H} 4$ test beam setup with respect to the CMS experiment is the absence of the $4 \mathrm{~T}$ strong magnetic field of the CMS solenoid and the absence of the material (vacuum tube and tracker) in front of the crystals. The later leads to additional corrections on the effects of bremsstrahlung, scattering and energy losses in this material.

Another important difference is that the ADC digitization clock is not synchronized with the APD signal of the interacting particles as it is in the case of LHC, where the signal phase is constant with respect to the bunch crossing. Therefore a Time to Digital Converter (TDC) was used to measure the phase between triggers and the ADC clock.

\subsection{Reconstruction of the signal amplitude}

As described earlier, the electrical signal from the photodetectors, which is a measure of energy deposited in the crystal, is amplified, shaped, and digitalized by VFE electronics at a frequency of $40 \mathrm{MHz}$. Thus, for each trigger the consecutive digitalizations within a defined time frame of $250 \mathrm{~ns}$, resulting in a series of 10 recorded samples, is read out for each event, see Fig. 6.5 left. Several amplitude reconstruction methods were developed to obtain an unbiased and precise estimation of the signal amplitude over the whole energy range, and tested during the last several test beam campaigns. Among them, a so called weights method has been chosen as the optimal one and now is used as a standard method in the ECAL. 

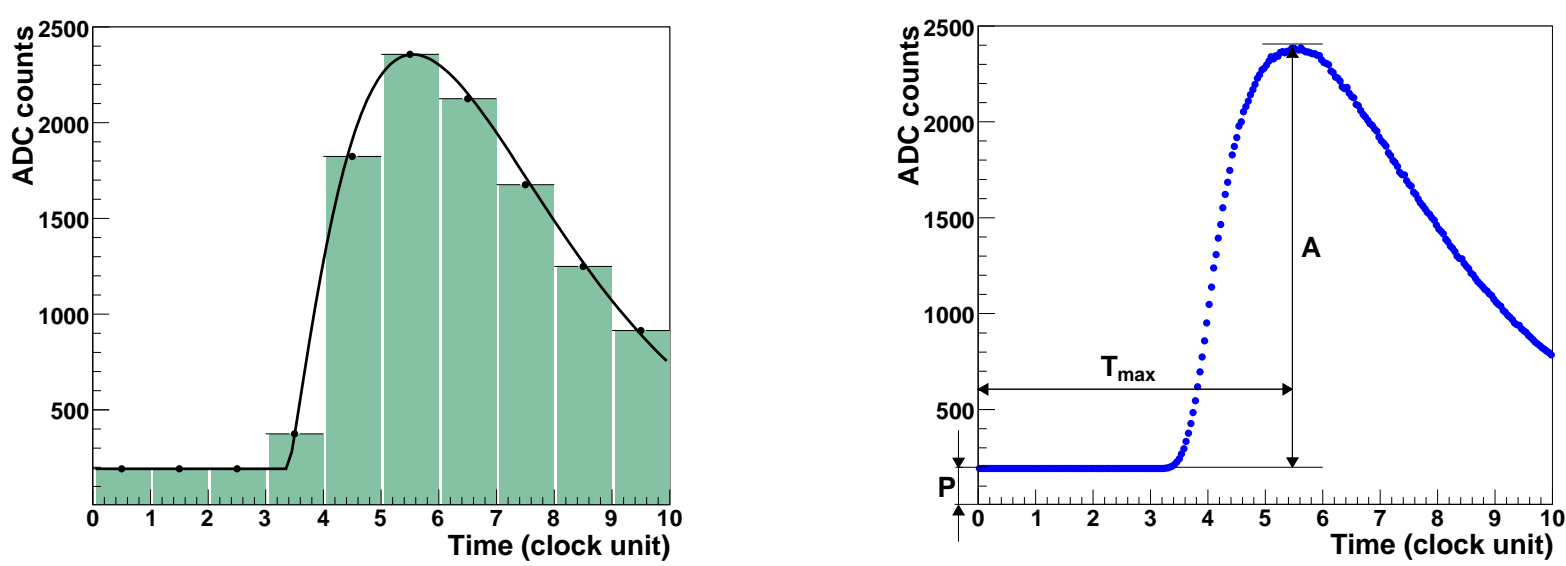

Figure 6.5: Left: Digital representation of the electronics pulse as a series of 10 samples. Each sample is recorded every $25 \mathrm{~ns}=1$ clock unit. The pedestal was not subtracted and the analytic fit represents a true shape of the signal pulse. Right: Profile histogram of the signal pulse obtained from a $120 \mathrm{GeV}$ electron run. The peaking time $T_{\max }$, the pedestal $P$, and the amplitude $A$ of the signal are also highlighted.

\subsubsection{The weights method}

The weights method [5] is based on a standard digital filtering technique that minimizes the noise contribution in the reconstructed amplitude and thus improves the signal to noise ratio. The amplitude is estimated as a linear combination of $N$ discrete time samples:

$$
\hat{A}=\sum_{i=0}^{i=N} S_{i} \times w_{i},
$$

where $\hat{A}$ is the estimate for the amplitude, $S_{i}$ are the individual time samples values in ADC counts composed by the signal, the noise and the pedestal, $w_{i}$ are the corresponding weights at time $t_{i}$, and $N$ is the number of samples used in filtering. The weights $w_{i}$ are determined by minimizing the expected variance of the reconstructed amplitude $\hat{A}$. Postulating that the estimator of the amplitude is equal to the amplitude $(E[\hat{A}]=A)$, which corresponds to an unbiased estimation of the true amplitude $A$, leads to the following constrains:

$$
\sum_{i=0}^{i=N} w_{i} f_{i}=1 \quad \text { and } \quad \sum_{i=0}^{i=N} w_{i}=0
$$

where $f_{i}=f\left(t=t_{i}\right)$ are the expected pulse heights. The amplitude is normalized to 1 . Therefore the determination of an optimal set of weights requires knowledge of a function $f(t)$ describing the time development of the signal pulse.

The function $f(t)$ is delivered from a digital representation obtained from test beam data. An example of such a profile histogram of the pulse shape build from a $120 \mathrm{GeV}$ electron data is displayed in Fig. 6.5 right. The weights are then extracted from the minimization of the expected 
variance estimated by the $\chi^{2}$ from observed and expected pulse samples:

$$
\chi^{2}=\sum_{i, j}\left(S_{i}-G_{i}\right) \times \operatorname{Cov}_{i, j}^{-1} \times\left(S_{j}-G_{j}\right)
$$

where $S_{i}$ is a measured time sample in ADC counts recorded at the time $t_{i}, G_{i}\left(A, P, T_{\max }\right)$ is the function that depends on different parameters: $A$ the true amplitude, $P$ the pedestal, and $T_{\max }$ the peaking time (see Fig. 6.5). Cov $_{i, j}$ is the noise covariance matrix, which represents the noise correlation between the time samples.

In the simplest case, when there is no correlated noise, the pedestal and the peaking time are known, so that $G=G(A)=A f(t)$ and the second condition in Eq. 6.2 can be omitted, the optimal weights, which give the best estimation of the amplitude $A$, are:

$$
w_{i}=\frac{f_{i}}{\sum_{j}^{N} f_{j}^{2}} .
$$

However, the test beam proved that also other parameters such as a signal shape and timing, depending on the type of the analysis and required precision of the energy resolution determination, play an important role and different degrees of the weights optimization are needed.

\subsubsection{Weights optimization}

The optimization of the weights method also includes the determination of how many samples and which ones should be used to obtain an optimal noise reduction factor and the most precise determination of the energy amplitude. Since the noise increases with the number of samples and, generally, the best noise reduction factor is achieved if the samples around the signal peak (the samples that carry most of the amplitude information) are considered. The amplitude in the ECAL will be reconstructed using 5 consecutive samples in the filtering: one sample before, one on the peak, and three after the peak. This configuration is here called the 5 weights method and can be applied in all three gains. In the highest gain 12 three pedestal pre-samples can also be taken and this configuration is called as the $3+5$ weights method with dynamic pedestal extraction. More information about noise optimization is given in Chap. 6.6.6.

Another important constraint in the reconstruction of the signal amplitude in the test beam condition, in comparison with the one in $\mathrm{LHC}^{3}$, comes from the fact that the test beam running is asynchronous, e.g. the phase between the signal of the interacting particle and its digitization is random. According to the type of the test beam study and its required precision, two different sets of weights were calculated and available: the standard and optimised weights [7] that will now be briefly described.

\section{Standard weights}

As a consequence of the asynchronous running, the signal pulse is sampled in different times with respect to the average $T_{\max }$ within a $25 \mathrm{~ns}$ phase. To correct this effect, 25 sets of weights are computed for each $1 \mathrm{~ns}$ bin of the phase and then an appropriate set is applied according to the information from the TDC. Thus, the time of the peak $T_{\max }$ becomes one of the most important parameters in weights optimization.

\footnotetext{
${ }^{3}$ If the setting of the channel timing is set correctly and the particle time-of-flight is taken in account, the synchronous running will require only one series of weights for an accuracy reconstruction of the energy over the whole ECAL. The small timing differences between channels will be absorbed by intercalibration [6].
} 
A considerable reduction of the set of weights can be achieved by means of an iterative timing alignment technique performed by laser measurement and timing offset determination from electron data. Laser dedicated runs were taken in order to determine the peaking time of each trigger tower which can be then adjusted iteratively until the dispersion among trigger towers timing is minimized. After the trigger tower timing alignment by the laser, the horizontal scan $(\eta=$ conts. $)$ using an electron beam with fixed energy across the center of 17 trigger towers was performed to determine the average timing offset in order to reach the average extracted peaking time.

This method allows to obtain the reduction of the trigger timing dispersion to less than $1 \mathrm{~ns}$ corresponding to a $6 \mathrm{~ns}$ peak-to-peak spread over all 1700 supermodules channels. This allows for the channels to be separated into one of three \pm 1 ns groups with a similar pulse profile for which only 3 different set of weights are then needed to be produced. The technique is fast and can allow to obtain the energy resolution $(\sigma / E(120 \mathrm{GeV}) \approx 0.48 \%)$ sufficient for the supermodule intercalibration. An example of the reconstructed amplitude in the $3 \times 3$ crystal array of the supermodule SM16 around 25 different crystals for $120 \mathrm{GeV}$ electrons using $3+5$ configuration with standard weights is shown in Fig. 6.6 left.
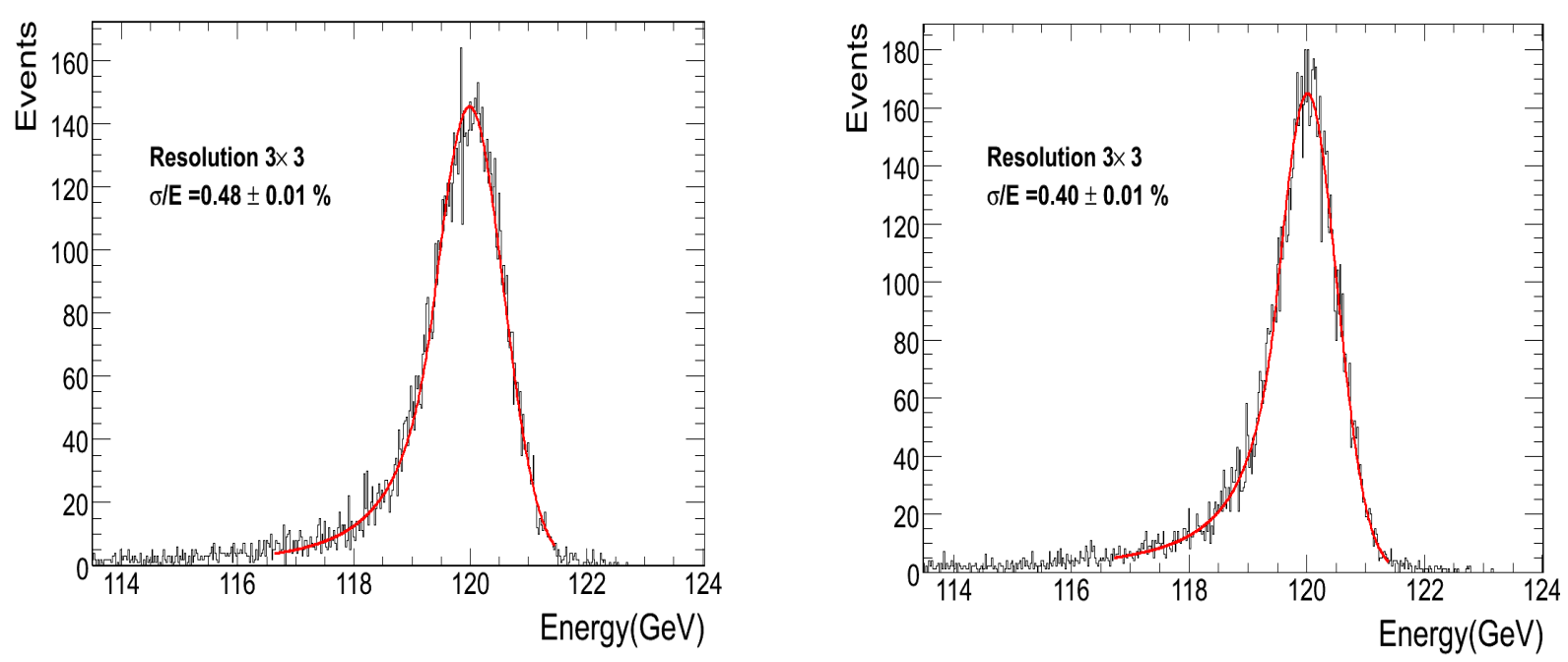

Figure 6.6: Energy reconstructed in the $3 \times 3$ crystal arrays with the beam centered on each of the 25 crystals computed by the use of standard (left) and optimized (right) weights.

\section{Optimized weights}

The optimized set of weights taking into account the slight differences between single crystals and thus giving the best energy resolution can be computed only for crystals for which a signal pulse profile can be precisely determined. To obtain the best performance, the weights are determined for each $1 \mathrm{~ns}$ phase bin. Due to the drastic reduction in the statistic (up to $93 \%$ of the origin of number of events) when only events satisfying a narrow hodoscope window (usually $4 \times 4 \mathrm{~mm}^{2}$ ) are considered for analysis, the high statistic electron run $(>100,000$ events $)$ is needed. This requirement highly limits the number of channels for which the profile and consequently the weights can be precisely determined. 
Therefore, a new technique which allows to produce a precise determination of the signal amplitude with a limited statistic was introduced in the 2006 test beam campaign. The method is based on the normalization of each entry of the profile histogram to obtain sufficiently smooth signal representation which is then used for the weights computation. The renormalization is performed by the use of the analytical function on each 1 clock profile interval to determine bias and thus correction factors for each entry over this $25 \mathrm{~ns}$ range. The corrections are applied for each event and the residual bias is removed by subsequent shape smoothing. The method was proved as robust and can be successfully used to obtain the optimized weights using less statistics $(\sim 30,000$ events per run) or even with intercalibration electron data $(\sim 2,000$ events per run). The performance of the method is clearly demonstrated in Fig. 6.6 right, where the amplitude for $120 \mathrm{GeV}$ is reconstructed as in the previous case, but using the optimized weights. The obtained energy resolution $\sigma / E(120 \mathrm{GeV})=0.40 \pm 0.01 \%$ instead of $0.48 \pm \%$ determined with the standard weights [6].

The energy resolution as a function of energy was estimated on a same matrix of 25 crystals and applying the same optimized weights computed at $120 \mathrm{GeV}$ electrons on several energies from 20 to $120 \mathrm{GeV}$ as is shown in Fig. 6.7. The parameter of the fitted function (see Fig. 6.7) demonstrates an excellent intristic energy resolution which can be obtained in the ECAL.

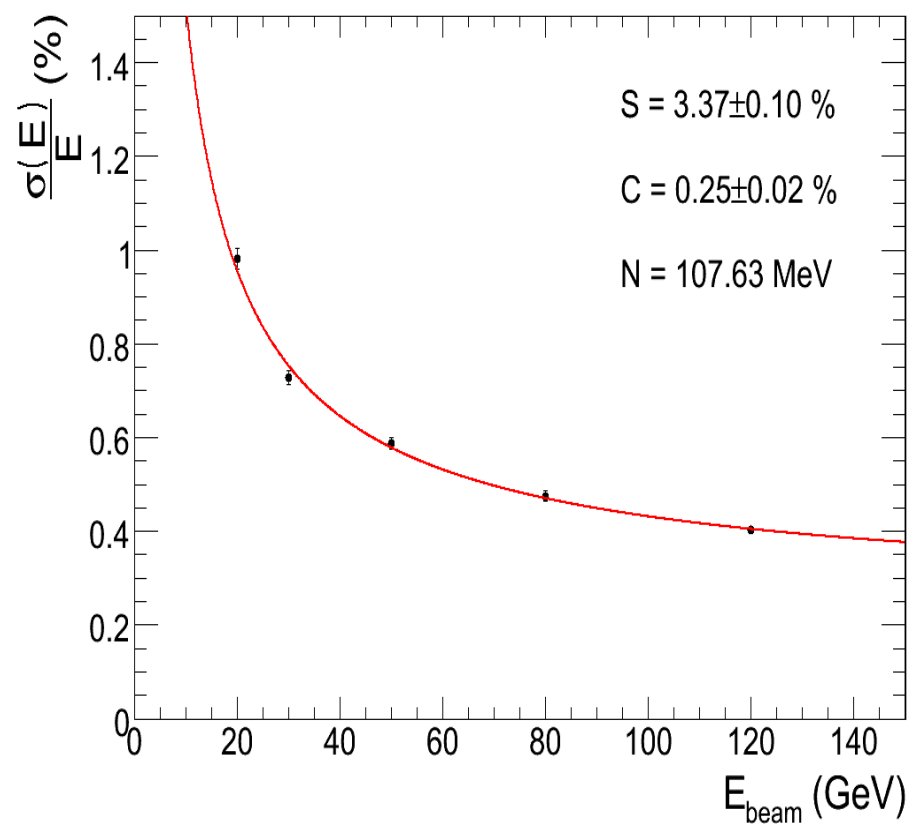

Figure 6.7: Energy resolution as a function of energy measured with an optimal set of weights in the crystal arrays $3 \times 3$ with the beam centered of each of 25 crystal arrays on the supermodule SM16 during the testbeam campaign 2006 [6].

\subsection{Noise Performance}

The most stringent physics requirements are imposed by the promising $H \rightarrow \gamma \gamma$ decay channel, which demands a superior energy resolution of the ECAL also for a relatively low energy range 


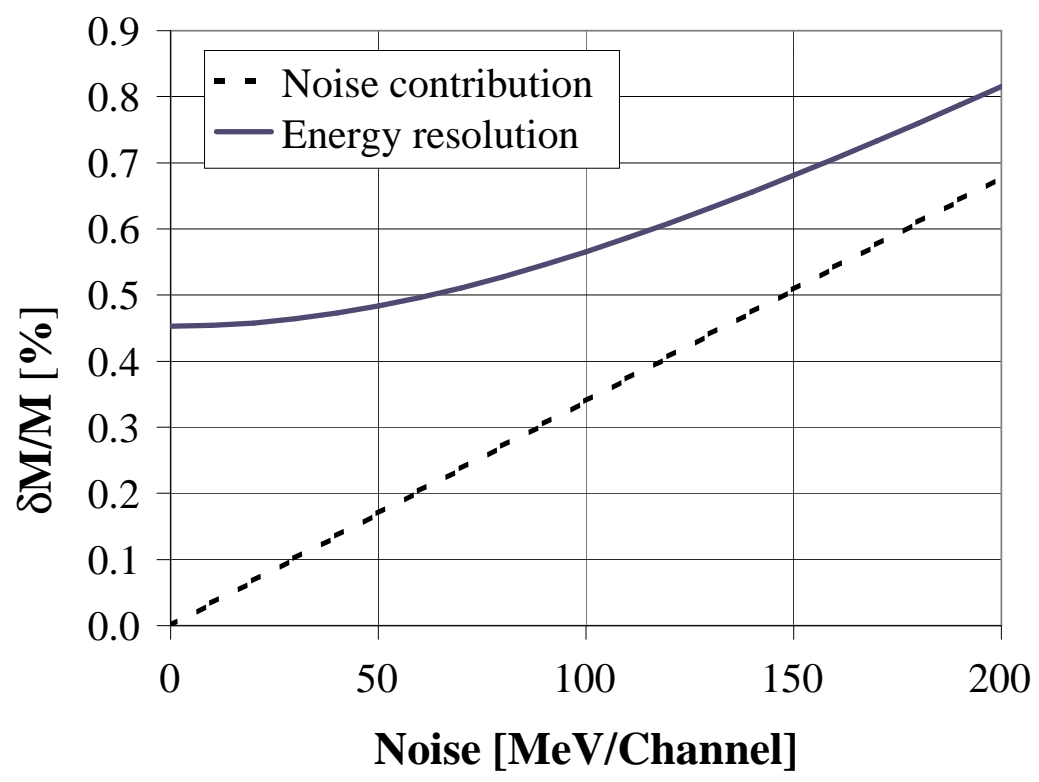

Figure 6.8: Noise contribution to the $H \rightarrow \gamma \gamma$ mass resolution for $m_{H}=100 \mathrm{GeV}$ [1].

$(\sim 50 \mathrm{GeV})$, where the noise contribution, including the electronics and pile-up noises, becomes quite important. Fig. 6.8 [1] demonstrates degradation of the $\gamma \gamma$ mass resolution that begins to be significant for noise greater than $\sim 50 \mathrm{MeV}$ per channel. This requires to keep the noise as low as possible and to monitor it regularly during the ECAL operation.

Electronics noise and pile-up noise are two main contributions to the noise term of the energy resolution expressed by Eq.2.1. The pile-up noise becomes significant only for the highest $\eta$ regions and at high luminosities and is mentioned here only for completeness.

\subsubsection{Pile-up noise}

The pile-up noise is due to the so called pile-up events, which occur if additional particles are recorded together with the signal of desired particles. Unwanted particles can be high energy particles generated with low probability which are shifted by a fixed multiple of a bunch crossing time $^{4}$ with respect to the amplitude signal, or low energy particles that can be recorded at any time because of the spiral inside the magnet.

The later are difficult to distinguish from the electronics noise and have a tendency to slightly increase the global noise. On the other hand, the high energy pile-up events form tails (see Fig.6.9) and therefore can be identified and reduced by an appropriate selection performed by cuts on the fit $\chi^{2}$ and $\Delta P$ which corresponds to the difference between the reconstructed and expected baseline evaluated from pedestal runs.

The effect of the pile-up noise was simulated using minimal bias events generated between -5 and +3 bunch crossing before and after signal with an average number of minimum bias events per bunch crossing of 3.5. The reconstructed amplitude for any signal without and with the

\footnotetext{
${ }^{4}$ The shaped signals of the VFE electronics is extended over several bunch crossing.
} 
pile-up noise at low luminosity $\left(\mathcal{L}=2 \times 10^{33} \mathrm{~cm}^{-2} \mathrm{~s}^{-1}\right)$ is shown in Fig.6.9 which demonstrates that its contribution to the noise at low luminosity is small [5].

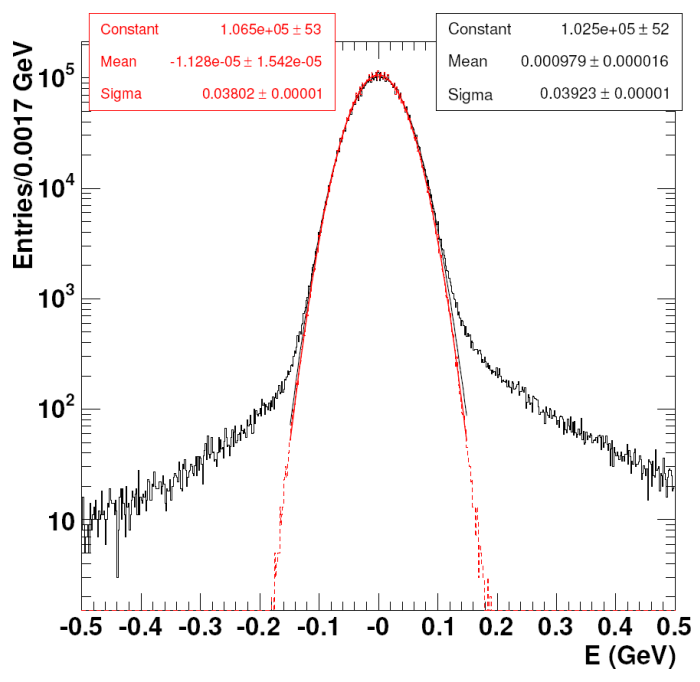

Figure 6.9: Reconstructed amplitude in ECAL barrel channels in the absence of a signal, without pile-up (red dashed distribution) and with pile-up (black distribution). A Gaussian of width of $40 \mathrm{MeV}$, representing the electronic noise, is superposed on the dashed distribution [5].

\subsubsection{Electronics noise}

The electronic noise in this study is taken as the total noise of the complete readout chain that can only be measured in real data taken conditions of the large scale system, such a supermodule or in large part of the endcap. Therefore the accurate measurements of the noise have been done during the last test beam campaigns 2006 and 2007 for the barrel and the endcap, respectively.

Since the contribution of the electronic noise ${ }^{5}$ is the main part in the noise term of the energy resolution, it was necessary to perform an accurate noise analysis study including the development of a fast and efficient evaluation technique, which provides identification of possible noise problems and thus allow to reduce their influence on the ECAL performance.

The noise performance was measured by means of pedestal runs, e.g. special runs intended to measure the electronic baseline (pedestal) on which an electrical signal is carried. The precise knowledge of the pedestal is required by the amplitude reconstruction procedure in which the pedestal has to be extracted from the signal amplitude. The pedestal is set for each gain separately and its value is programmable using a simple DAC circuit internal to the MGPA which is controlled externally by an $I^{2} C$ interface. Usually, several hundreds of pedestal events are used for each gain with a mean value set to be around $200 \mathrm{ADC}$ counts. Once the pedestal is set, its actual value must be measured.

There are two ways to measure the pedestal in situ. Generally, the pedestal can be estimated by means of pre-samples (so called dynamic determination of the pedestal) recorded before the

\footnotetext{
${ }^{5}$ The electronic noise will be referred in this chapter simply as noise.
} 
rising edge of the signal amplitude. This method allows to extract precise pedestal value for each event, but only for the highest gain 12. Due to the automatic gain switching for higher energy, there are no pedestal samples for the middle and low gains. The second method of pedestal determination uses dedicated pedestal runs, which allows to estimate pedestal value separately for each gain and thereby to also measure the electronic noise for each gain.

For this reason, the accurate analysis of the pedestal measurement is very important for ECAL amplitude reconstruction for high energy events because the dynamic extraction of pedestal can be used only for the highest gain 12, the pedestal value for gain 6 and 1 will be estimated from a periodically measured pedestal for each channel.

\subsubsection{Noise evaluation definitions}

The total noise is the measure of the fluctuation of each sample value with respect to the mean pedestal value and can be defined as a standard deviation $\sigma_{T o t}$ of the reconstructed amplitude when no signal is input, it is computed as:

$$
\sigma_{T o t}=\sqrt{\frac{1}{N} \sum_{i}^{N}\left(S_{i}-\frac{1}{N} \sum_{i}^{N} S_{i}\right)^{2}}
$$

where $S_{i}$ are individual measured time sample values in ADC counts and $N=n \times m$, where $n$ is the number of recorded events in each gain and $m$ is number of time samples per event.

The total noise consists of two comparable components: low frequency noise and high frequency noise. Low frequency noise $\sigma_{L F}$ represents fluctuation between events (mean value of pedestal for each event with respect to the mean value of the pedestal over all recorded samples) with a period greater than a high frequency noise $\sigma_{H F}$ that corresponds to fluctuations between samples of the same event having a period of $250 \mathrm{~ns}$. Then the total noise can be expressed as quadratic summation of its individual contributions:

$$
\sigma_{T o t}=\sigma_{L F} \oplus \sigma_{H F},
$$

where low and high frequency noises are defined as:

$$
\begin{gathered}
\sigma_{L F}=\sqrt{\frac{1}{n} \sum_{j}^{n}\left(\frac{1}{m} \sum_{k}^{m} S_{j k}-\frac{1}{N} \sum_{i}^{N} S_{i}\right)^{2}} \\
\sigma_{H F}=\sqrt{\frac{1}{N} \sum_{j}^{n} \sum_{k}^{m}\left(S_{j k}-\frac{1}{m} \sum_{k}^{m} S_{j k}\right)^{2}}
\end{gathered}
$$

An example of the noise evaluation in accordance with above mentioned definitions with its typical values measured for a single channel in highest gain 12 are displayed in Fig. 6.10.

\subsubsection{Systematic studies of pedestal end electronic noise}

Systematic studies of all pedestal runs taken during the test beam campaigns in 2006 on 9 supermodules and 2007 on one endcap array containing 500 crystals were performed. In Fig. 6.11 presents the results for the total noise performance for one typical pedestal run taken on barrel supermodule SM17 and the endcap matrix, both for gains 12, 6, and 1, respectively. 

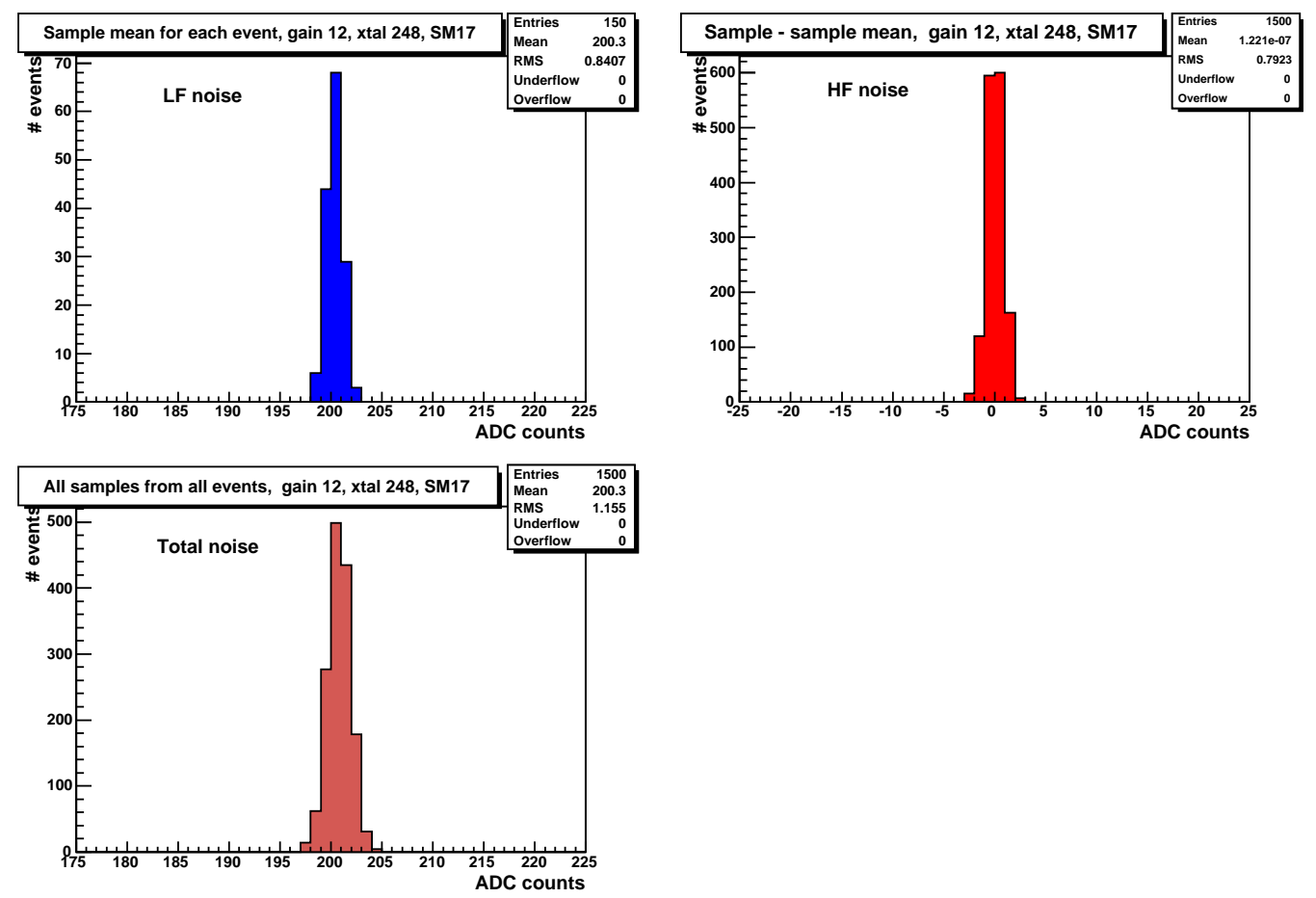

Figure 6.10: Top left: Low frequency noise $\sigma_{L F}=0.84 \mathrm{ADC}$ counts as a standard deviation of the distribution of the mean sample values of each event (150 entries). Top right: High frequency noise $\sigma_{H F}=0.79 \mathrm{ADC}$ counts as a standard deviation of the distribution of the difference of each sample value and the mean sample value over the 10 samples in the event (1500 entries). Bottom left: Total noise $\sigma_{T o t}=1.16 \mathrm{ADC}$ counts as a standard deviation of the distribution of the all time sample values of all the events $(10 \times 150=1500$ entries $)$.

\section{Total noise}

In Fig. 6.11, the noise shows similar behaviour over all the channels with dispersion of several percent. The noise for the highest gain is around 1 and 2 ADC counts for the barrel and endcap, respectively. The difference in noise magnitude is due to different dynamic ranges of VFE electronics for the barrel and endcaps. The different in the mean value of the noise for each gain is due to the external components which set a dynamic range of the readout electronics.

One has found in the barrel a channel (for instance a channel 934 in Fig. 6.11) whose pedestal as well as noise are equal to zero. If such a channel doesn't respond to laser impulsion or impinging particles or it responds with unpredictable values, it is marked as a dead channel in the database and it is excluded from the analysis. It needs to be noted that in spite of an extensive screening procedure, by which the reliability per channel is close to $100 \%$, a modest number of dead channels are expected $(0.1 \%)^{6}$.

\footnotetext{
${ }^{6}$ At present, in situ (36 supermodules), around 80 barrel channels have been found noisy or dead.
} 

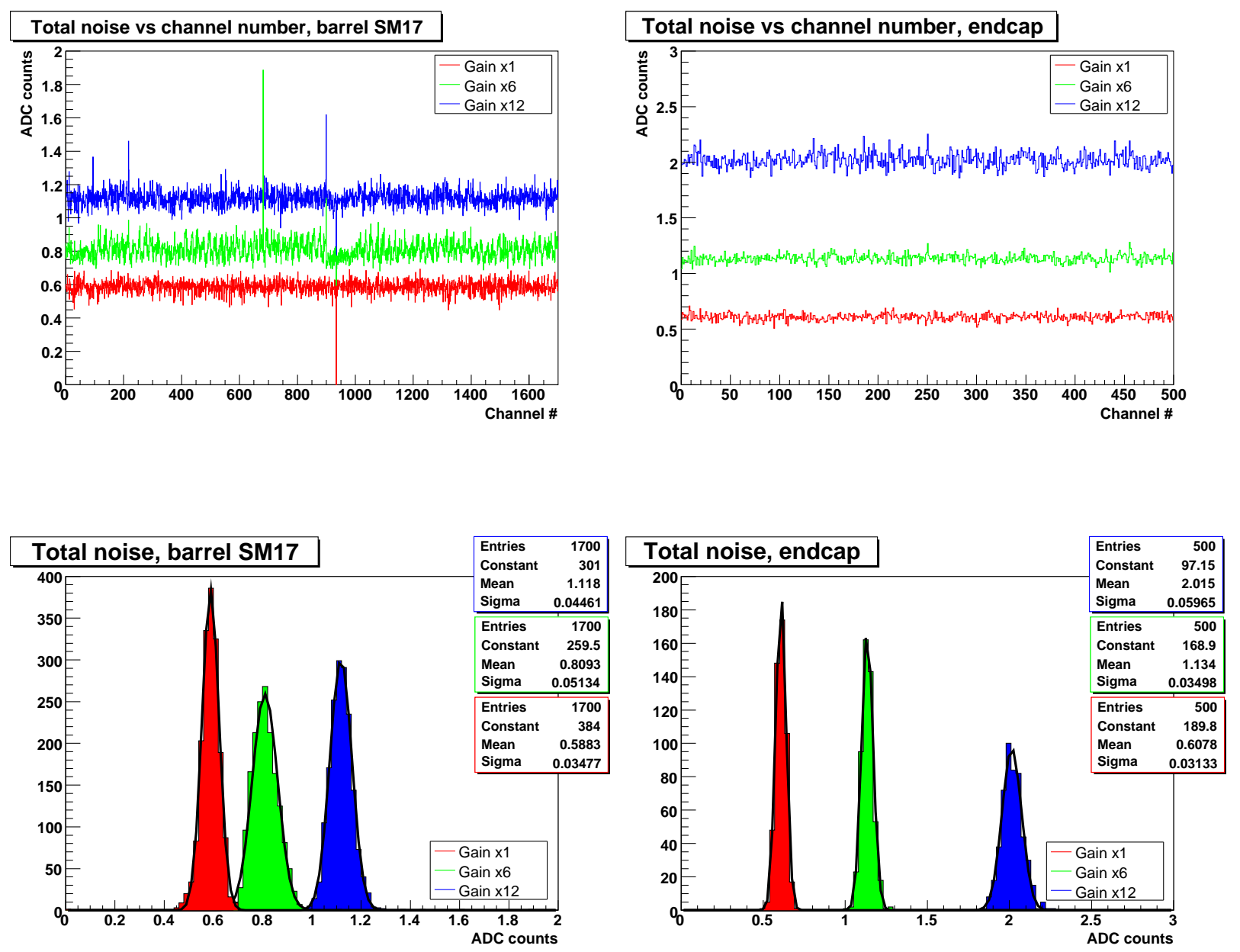

Figure 6.11: Top figures: Total noise in ADC counts as a function of channel number for the three gains 12, 6, and 1 for the barrel supermodule SM17 (left) and the endcap 500 crystal array (right). Bottom figures: Distribution of the total noise in ADC counts for the three gains for all the SM17 channels and 500 channels of the endcap.
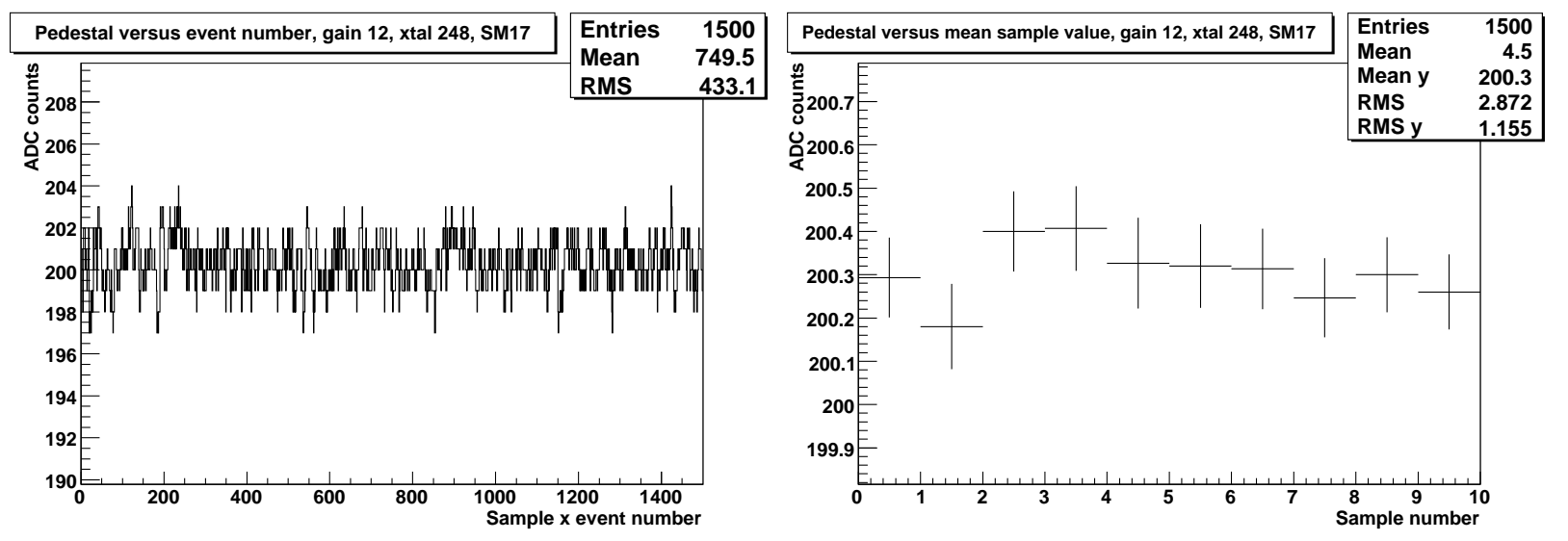

Figure 6.12: Left: Mean pedestal value as a function of the time sample for all the events. Right: Mean pedestal value over all events as a function of time sample. Both figures are for gain 12, channel 248, supermodule SM17 and show normal behaviour. 
Moreover, in the barrel, several channels with slightly evaluated noise values for middle and highest gain are present. It clearly proves that abnormal behaviour of the noise needs to be evaluated for each gain separately. In such a case, the problematic channels are identified and undergo a detailed study to find the origin of the problem. This step includes a check if the sample values for all events remain in a predefined window, or if the problem is related to particular time samples (see Fig. 6.12). The main aim of this analysis is to decide if the problem is significant for the amplitude reconstruction or not. If the amplitude reconstruction is affected, it has to be checked if the channel behaves abnormally only for particular pedestal run/runs or if a problem has a long term character. The temporary pedestal problem can be, for example, due to a corrupted run during the data acquisition or data storage, etc. The long term problems are more serious and in this case, the channel and its behaviour needs to be reported in the database for possible special treatment in other test beam studies.

\section{Low and high frequency noise}

Analysis of the low and high frequency components gives a direct indication to know if the possible noise problem is on the sample or on the event base. As an example of total noise distribution and its two components for the supermodule SM17 and the part of the endcap for gain 12 is displayed in Fig. 6.13, where one can see that the mean values of low and high frequency noise is around the same value for the barrel contrary to the endcap, where the high frequency noise is a bit higher. The same effect is also seen for the middle gain (see Fig. 6.14), in gain 1 both components behave similarly.
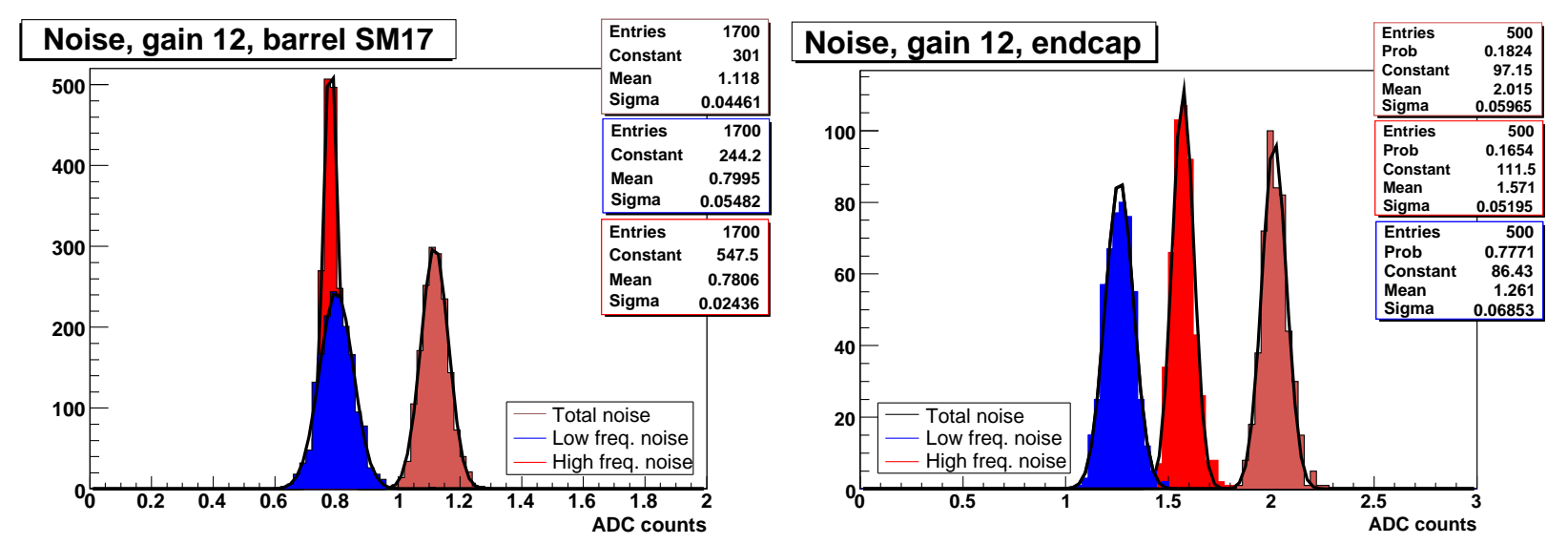

Figure 6.13: Distribution of the total, low and high frequency noise for gain 12 measured for all the 1700 channels of the supermodule SM17 (left) and 500 endcap crystal array (right).

\subsubsection{Correlated noise}

Two different correlations were studied during the test beam campaigns. The first type is the spatial correlated noise between channels. Its evaluation is important because of the energy reconstruction algorithm which computes the deposited energy as a sum in $3 \times 3$ or $5 \times 5$ crystal arrays. The second type is the time correlated noise which is seen between amplitude time samples of the same channel. 

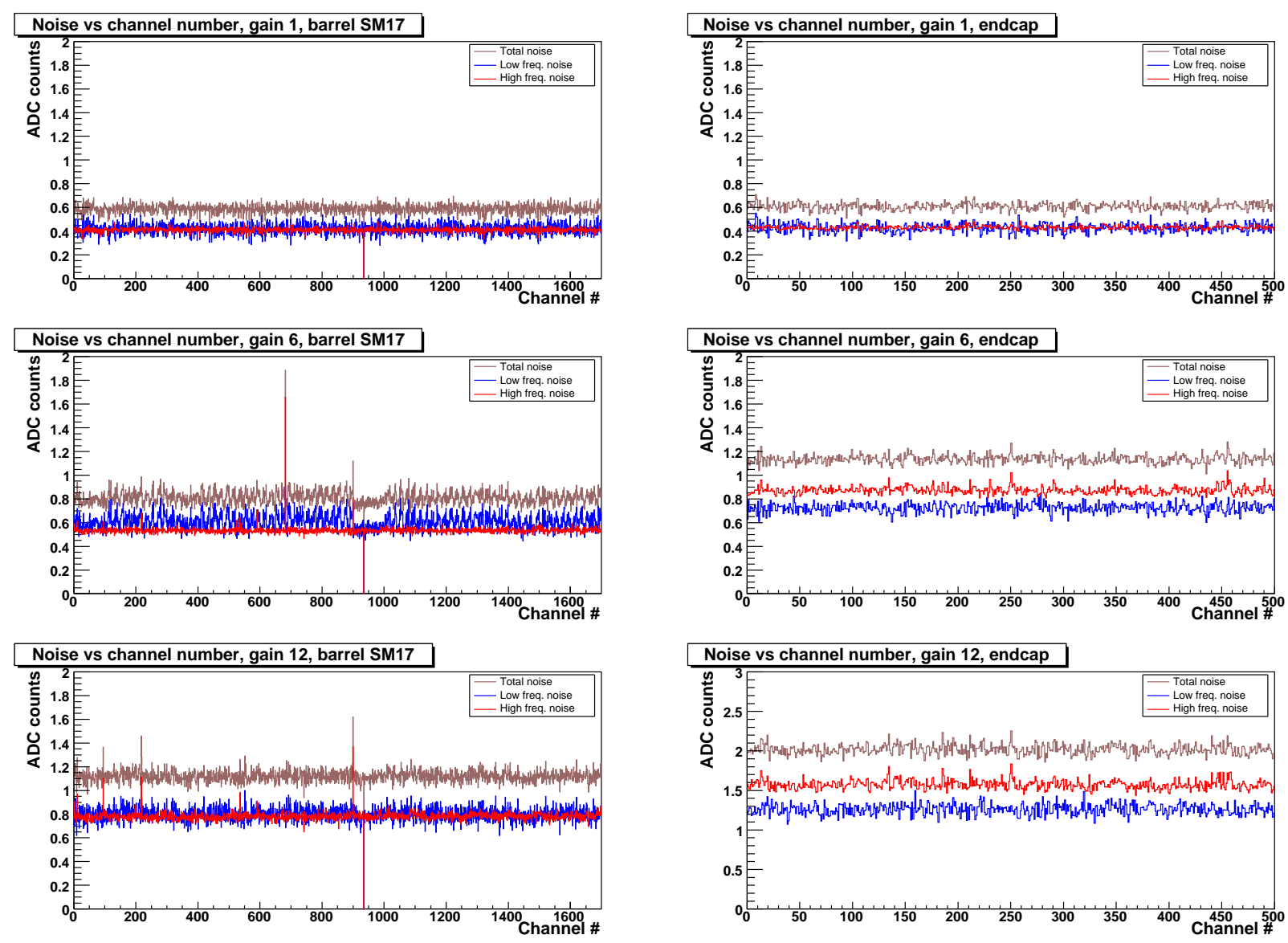

Figure 6.14: Total, low and high frequency noise for three gains 12, 6, and 1 as a function of channel number measured on the supermodule SM17 (left) and on the 500 endcap crystal array (right).

\section{Spatial correlated noise}

Strong correlated noise between channels has been observed on the supermodule prototypes SM1 and SM10 during the previous test beam campaigns $[8,9]$. The spatially correlated noise was uniform over the studied channels and related only to low frequency components. Therefore, it was necessary to remove it effectively by estimating of the low frequency noise evolution from the first two samples of each event taken in pedestal run and subsequently applying the correction on all samples [9].

In the definitive version of the supermodule with the final read-out electronics and due to different grounding, the coherent noise has been improved and can be completely suppressed by the amplitude reconstruction method with dynamic pedestal subtraction. The effectiveness of this procedure is clearly demonstrated in Tab.6.1 (first line), where the total noise in the sum of $25(5 \times 5)$ or $9(3 \times 3)$ channels is almost exactly 5 or 3 times the single channel noise, respectively.

The robustness of the above mentioned method was proved for all the barrel supermodules 
and the endcap crystal array by evaluating the noise for each channel computed as a sum of its neighbouring channels, for both $3 \times 3$ and $5 \times 5$ crystal arrays. An example of the noise in the sum of 9 and 25 channels over whole supermodule SM17 is shown in Fig. 6.15. In comparison with the single channel noise (see. Fig $6.11 \mathrm{left}$ ) only a small contribution of the channel-to-channel correlated noise is seen.
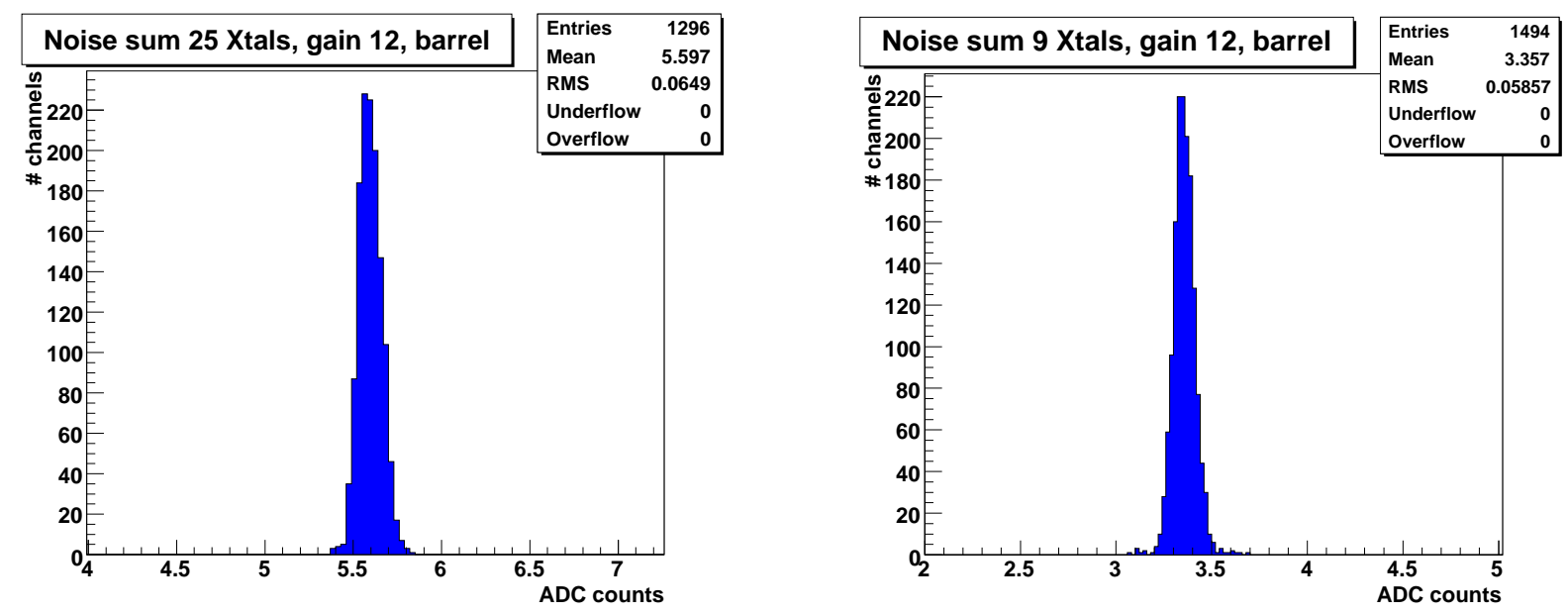

Figure 6.15: Distributions of the total noise in ADC counts in the sum of 25 (left) and 9 (right) over all channels in supermodule SM17 measured in gain 12.

\begin{tabular}{|c|c|c|c|c|c|c|}
\hline \hline Method & \multicolumn{3}{|c|}{ Barrel } & \multicolumn{3}{c|}{ Endcap } \\
$n+m$ & $1 \times 1$ & $3 \times 3$ & $5 \times 5$ & $1 \times 1$ & $3 \times 3$ & $5 \times 5$ \\
\hline 1 Sample & 1.12 & 3.36 & 5.62 & 2.00 & 6.02 & 10.04 \\
\hline $0+5 \mathrm{~W}$ & 1.14 & 3.43 & 5.75 & 1.91 & 5.73 & 9.57 \\
$3+5 \mathrm{~W}$ & 1.05 & 3.15 & 5.26 & 2.40 & 7.22 & 12.06 \\
\hline $3+1 \mathrm{~W}$ & 1.07 & 3.21 & 5.36 & 2.31 & 6.93 & 11.57 \\
$4+1 \mathrm{~W}$ & 1.05 & 3.16 & 5.27 & 2.27 & 6.84 & 11.41 \\
$5+1 \mathrm{~W}$ & 1.04 & 3.12 & 5.21 & 2.26 & 6.76 & 11.29 \\
$6+1 \mathrm{~W}$ & 1.03 & 3.10 & 5.16 & 2.23 & 6.71 & 11.18 \\
$7+1 \mathrm{~W}$ & 1.02 & 3.07 & 5.12 & 2.20 & 6.61 & 11.03 \\
\hline \hline
\end{tabular}

Table 6.1: Electronic noise in ADC counts measured in a single channel and arrays of $3 \times 3$ and $5 \times 5$ crystals as an average value over the entire supermodule SM17 and the endcap 500 crystal array for a given pedestal run. $n+m$ describes the weights method configuration, where $n$ is the number of weights for pre-samples and $m$ is a number of weights applied to the peak samples. 


\section{Time correlated noise}

In order to quantify the time correlation, the noise correlation matrix $\operatorname{Corr}_{i, j}=\frac{\operatorname{Cov}_{i, j}}{\sigma_{i} \sigma_{j}}$ was determined for every single channel. To reduce statistical error, each matrix element represents a mean value over all events in a given pedestal run. The covariance can be written as:

$$
\left.\operatorname{Cov}_{i, j}=E\left[n_{i} \times n_{j}\right]=E\left[\left(S_{i}-P_{i}\right)\right)\left(S_{j}-P_{j}\right)\right],
$$

where $n_{i}$ is the difference between the sample value $S_{i}$ and its pedestal $P_{i}$. It is clear that the diagonal elements are equal to the squared single sampling noise $\operatorname{Cov}_{i, i}=\sigma^{2}$.
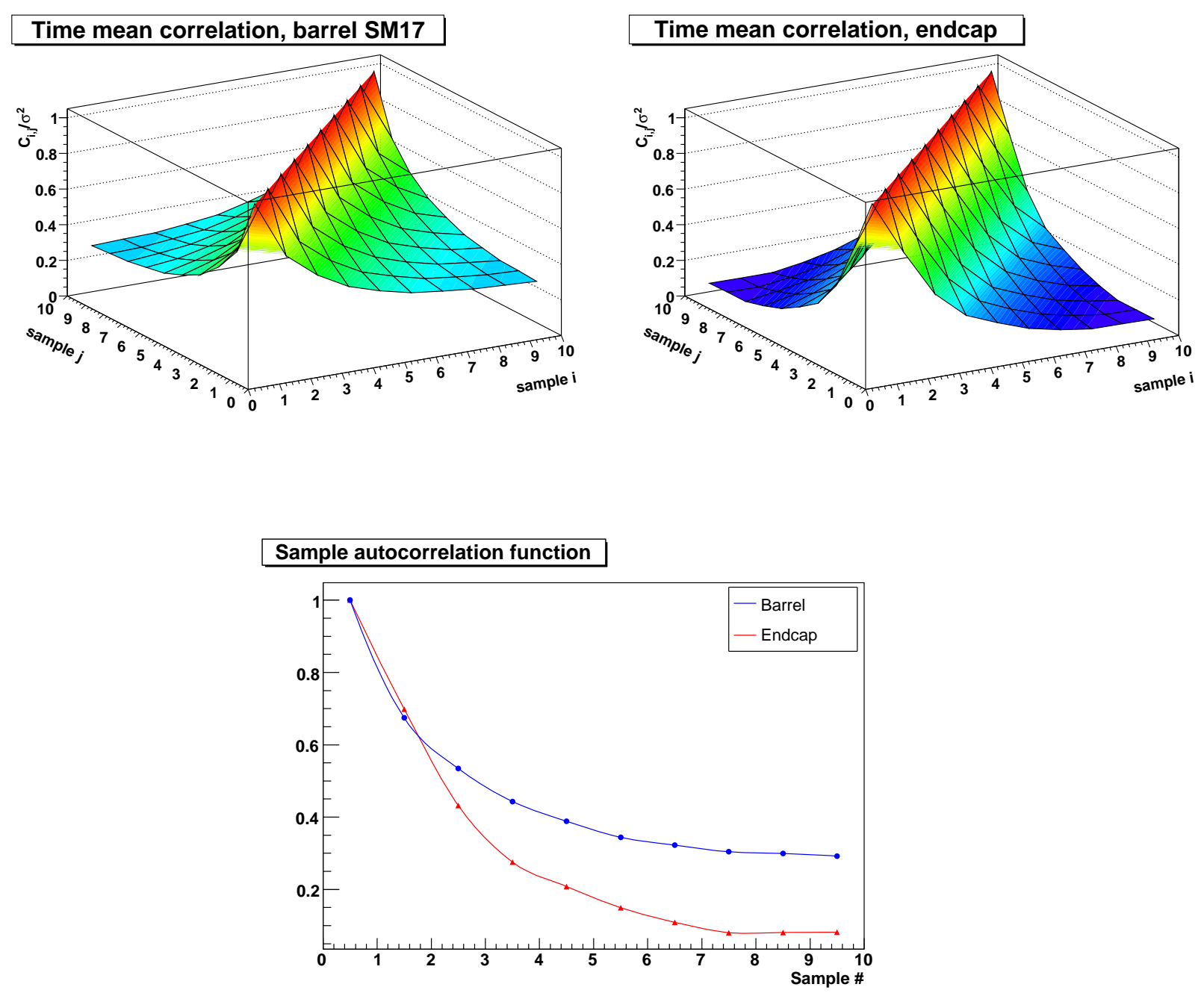

Figure 6.16: Noise correlation matrix showing the correlation of the noise between samples as a mean value over all channels of the barrel supermodule SM17 (top left) and the endcap 500 crystal arrays (top right). The bottom figures represent the corresponding autocorrelation function for the barrel as well as the endcap. The lines are only to guide the eye.

The matrices for all channels are very similar, thus it is possible to compute a mean correlation matrix over all the channels. Fig. 6.16 (top figures) shows the mean correlation matrices for 
the supermodule SM17 and the 500 endcap crystal array and (bottom figure) the corresponding autocorrelation functions. In both cases, one can see strong correlations between samples close in time which is due to the sampling period that is shorter than the electronics shaping time. Thus the correlated noise decrease with increasing times interval between the sample pair as the influence of the parallel noise, which is the main part of the MGPA preamplifier noise in the highest gain 12. Finally, the correlated noise drops to the residual value which corresponds to the low frequency noise, which is around $40 \%$ greater in endcap than in barrel for the highest gain 12 as is shown in Fig. 6.13. The less correlated noise in the endcap results in a stronger high frequency noise.

The measured noise covariance matrix can also be used with the weights method of the amplitude reconstruction to compute an optimized set of weights which will thus take into account the noise correlated between the samples. It was found [10] that a better noise reduction factor is obtained when the covariance matrix is used, but the benefit is relatively small compared to the significant improvement obtained using the pedestal subtracting weights method without the matrix implementation. For that reason and to keep the application of the weights method simpler and faster, the noise covariance matrix is not used in the standard amplitude reconstruction.

\subsubsection{Noise reduction in the amplitude reconstruction}

The amplitude reconstruction method that uses a digital filter is an efficient method to precisely determine signal amplitude and to minimize noise contribution. To obtain the maximal benefit of the method, the optimal set of weights have to be determined as described in chap. 6.5.2. This also includes optimization on the noise reduction.

The noise reduction in the amplitude reconstruction depends on the precision of the pedestal determination and also of the number of samples used in the filtering. As already shown, the pedestal can be precisely determined on the event basis using several samples ahead of the signal (pre-samples). Thus the study of a different number of samples used in the filter was performed for both the barrel and endcap. In this study the time correlated noise was not taken into account and the noise covariance matrix was defined to be $\operatorname{Cov}_{i . j}=\mathbf{1} \sigma^{2}$.

The results, which are summarized in Tab. 6.1, are presented only for the highest gain 12 because the pre-samples can only be measured for this gain. The configuration is described as $n+m$ weights, where $n$ is the number of weights for pre-samples and $m$ is a number of weights applied to the peak samples. Two samples were considered between the last pre-sample and peaking samples. The most significant noise reduction is observed when more samples are used. But this is not true for the endcap, where the best noise reduction factor was obtained when no pre-sample was used as an effect of higher high frequency noise.

The impact on the noise reduction when the number of the considered pre-samples increased is evident for both the barrel as well as for the endcap. The noise reduction is due to increasing precision in pedestal determination and thus better reduction of the low frequency component of the electronics noise. Therefore, as an optimal configuration for the ECAL, giving the marginal noise reduction, the $3+5$ weights configuration has been chosen.

\subsubsection{Noise stability over time}

Stability of the pedestal and electronics noise over time has been monitored throughout all the test beam campaign for all nine supermodules and for the endcap crystal array, and found very stable at a percent level in both cases. One has removed the corrupted runs as well as those used 
for special studies. The evolution of the total noise and its two components for one supermodule SM6, the supermodule that was studied for the longest time during the test beam campaign, and the endcap 500 crystals array are displayed for all three gains in Fig. 6.17, where the time scale is represented by the run number.
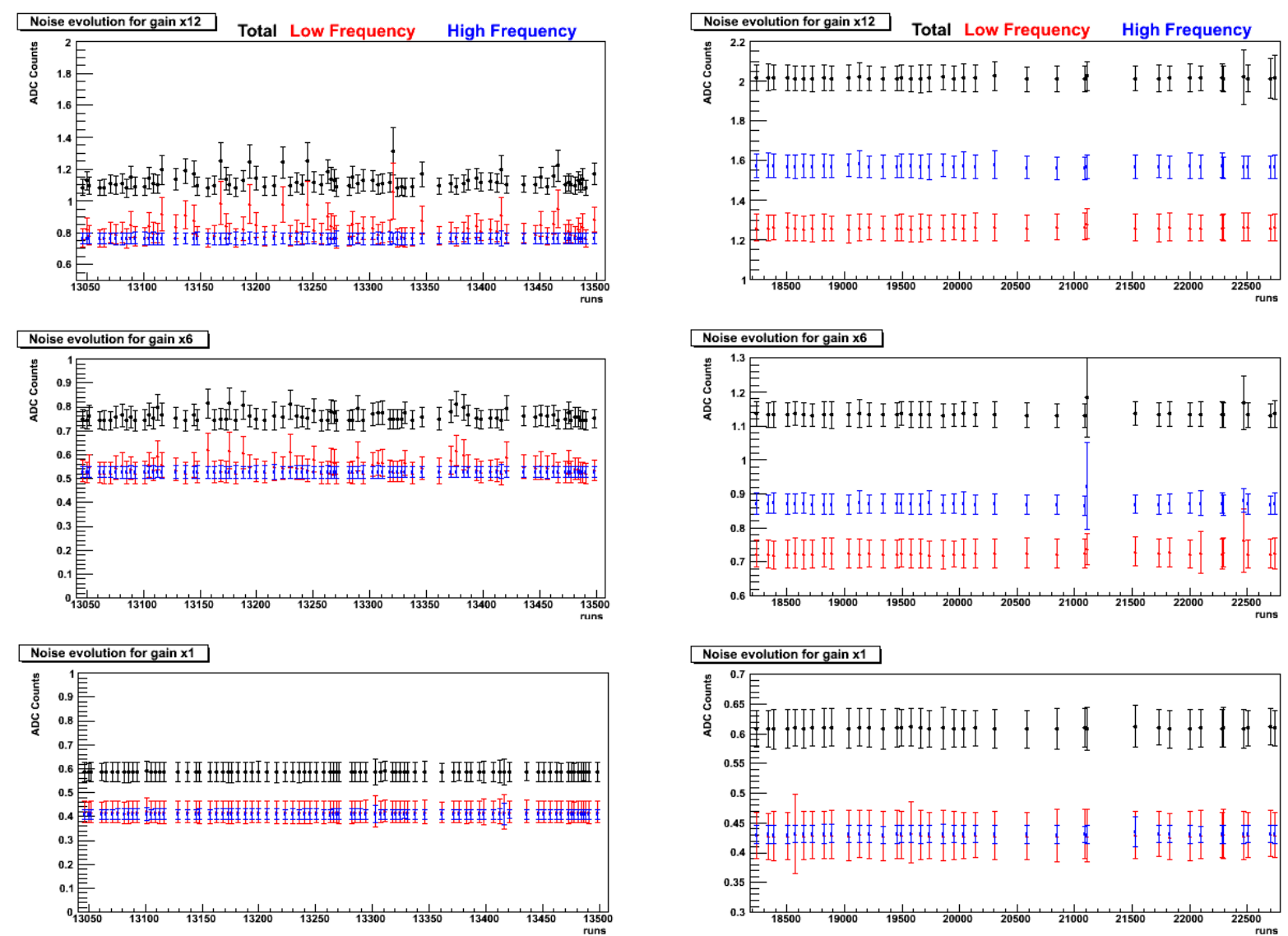

Figure 6.17: The total, low and high frequency noise for all three gains as a function of time (represented by a run number and cover a period of 3 weeks) for barrel supermodule SM16 and the endcap 500 crystal array. Each point is calculated as the average over all channels and error bars corresponds to the RMS value of this average.

In total, 82 and 39 pedestal runs were recorded over a period of 3 weeks for the barrel and endcap. The final results averaged over the entire considered period are summarized in Tab. 6.2. In the case of the barrel, the total noise for gain 12 is stable within $4 \%$ over the whole period. This relatively large number is due to a $7 \%$ fluctuation of the low frequency noise. The stability of the high frequency noise is better than 1 permille. A better stability was observed with the endcap, where the dispersion of total noise over all the studied period is within $0.2 \%$. 


\begin{tabular}{|ll|c|c|c|c|c|c|}
\hline \hline \multicolumn{2}{|c|}{$\begin{array}{c}\text { Noise } \\
{[\text { ADC counts] }}\end{array}$} & Gain 1 & Gain 6 & Gain 12 & Gain 1 & Gain 6 & Gain 12 \\
\hline Total & Mean & 0.59 & 0.76 & 1.13 & 0.61 & 1.14 & 2.02 \\
& RMS [\%] & 0.15 & 2.51 & 4.06 & 0.19 & 0.85 & 0.24 \\
\hline Low freq. & Mean & 0.42 & 0.55 & 0.82 & 0.43 & 0.76 & 1.26 \\
& RMS [\%] & 0.29 & 4.74 & 7.30 & 0.36 & 0.91 & 0.53 \\
\hline High freq. & Mean & 0.41 & 0.53 & 0.76 & 0.43 & 0.87 & 1.57 \\
& RMS [\%] & 0.11 & 0.08 & 0.07 & 0.17 & 1.01 & 0.31 \\
\hline \hline
\end{tabular}

Table 6.2: Average values of the total, low and high frequency noise measured in all three gains on the supermodule SM16 and the endcap 500 crystal array. The average noise was calculated over the whole studied period, see text.

\subsubsection{Conclusion on noise analysis}

The noise performance study during the test beam campaign shows that the electronic noise is very stable over the whole analyzed period and well under control. Efficient and robust noise evaluation tools were also developed and integrated into the CMSSW software and are now in common use. The total electronics noise in energy units ${ }^{7}$ in the barrel is about $39 \mathrm{MeV}$ for a single channel, $117 \mathrm{MeV}$ for the sum of 9 crystals and $194 \mathrm{MeV}$ for the sum of 25 crystals. This excellent noise performance fully satisfy the ECAL specifications. Moreover, the noise performance has been well optimized by using the appropriate amplitude reconstruction algorithm with the pedestal-subtracting weights method which efficiently reduces the low frequency noise to an insignificant level.

The noise performance and its stability was also evaluated in the endcap. The total electronics noise is higher by a factor of $\sim 3$ than in the barrel, its values are about $118 \mathrm{MeV}$ for a single channel, $355 \mathrm{MeV}$ for the sum of 9 crystals and $593 \mathrm{MeV}$ for the sum of 25 crystals. The higher values in the endcap are acceptable because the contribution of the noise term in higher energy is much less important.

\subsection{Gain ratio measurement}

The three analog output signals of the MGPA are digitalized in parallel by the ADC. Digital logic internal to the ADC determines whether a gain is saturated and then outputs the data from the highest non-saturated gain. In the barrel (endcap), the saturation of the highest gain occurs for a signal of approximately $150 \mathrm{GeV}(250 \mathrm{GeV})$. In order to reconstruct the amplitude at a higher energy, the relative gain of the two gain stages must be precisely determined. Fig. 6.18 [11] demonstrates the impact of the gain ratio on the energy resolution, where a $1 \%$ change in the gain ratio value results in a deterioration of almost $25 \%$ in energy resolution. This example demonstrates the importance of the gain ratio and thus the precision of its measurement.

For example, in the barrel, for energies above $180 \mathrm{GeV}^{8}$ the first sample, as in any case, is

\footnotetext{
${ }^{7} 1 \mathrm{ADC}$ count is approximately $37 \mathrm{MeV}$ and $62 \mathrm{MeV}$ in the barrel and endcap, respectively. These values can vary strongly according to the light yield value of the crystal considered.

${ }^{8}$ Due to the energy leakage, the energy deposited in a single crystal is smaller than the energy of impinging
} 


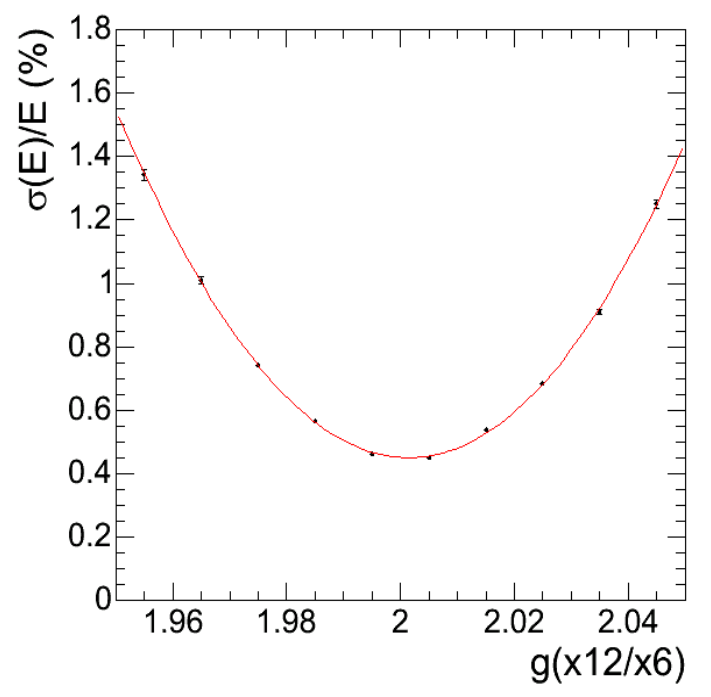

Figure 6.18: Energy resolution as a function of the gain ratio using test beam data with a $180 \mathrm{GeV}$ electron beam. The energy is reconstructed for a $3 \times 3$ crystal array [11].

taken in the highest gain 12 and following samples are recorded in the same gain until one sample reaches the saturation level of the ADC. Then the internal ADC logic switches to the middle gain 6 where the other consecutive samples are taken. If the deposited energy in the crystal is high enough, the gain 6 is switched to the low gain 1 for the rest of the samples.

An example of the signal pulse recorded over the highest and middle gains before and after gain ratio multiplication is demonstrated in Fig. 6.19 [13]. It is clear, that for amplitude reconstruction of energy deposited in the crystal, the gain ratios between two different gains must be known to provide a precise amplitude reconstruction.

Intercalibration of the ECAL is made at 90 or $120 \mathrm{GeV}$ and thus in the gain 12 range. If the precision of the measurement of the gain ratio can be made small compared to the target intercalibration precision of $\sim 0.5 \%$ then the impact on performance of the gain change would be negligible. Therefore, several methods of gain ratio measurement were developed and extensively tested on the fully-equipped supermodules during the 2006 test beam campaign at CERN. The results have been compared with those obtained from laboratory measurements performed on the VFE calibration bench at IPN Lyon where all the VFE cards have been calibrated and fully characterized [14].

All these techniques of the gain ratio measurements compute the gain ratio as the ratio of the mean value of signal amplitudes reconstructed in different gains for a same input pulse signal. For instance, the ratio of the amplitude reconstructed for a given input signal in gain 12 and gain 6 is equal to the relative gain between these two MGPA gain channels, and here will be reported as the gain ratio $12 / 6$. The amplitude can be measured by means of a MGPA test pulse charge, a laser light pulse of the optical monitoring system or scintillation light generated in the electromagnetic shower in a crystal.

particles. Fractions of the deposited energy are around 78, 94 and $97 \%$ for a $1 \times 1,3 \times 3$ and $5 \times 5$ crystal array, respectively [12]. 

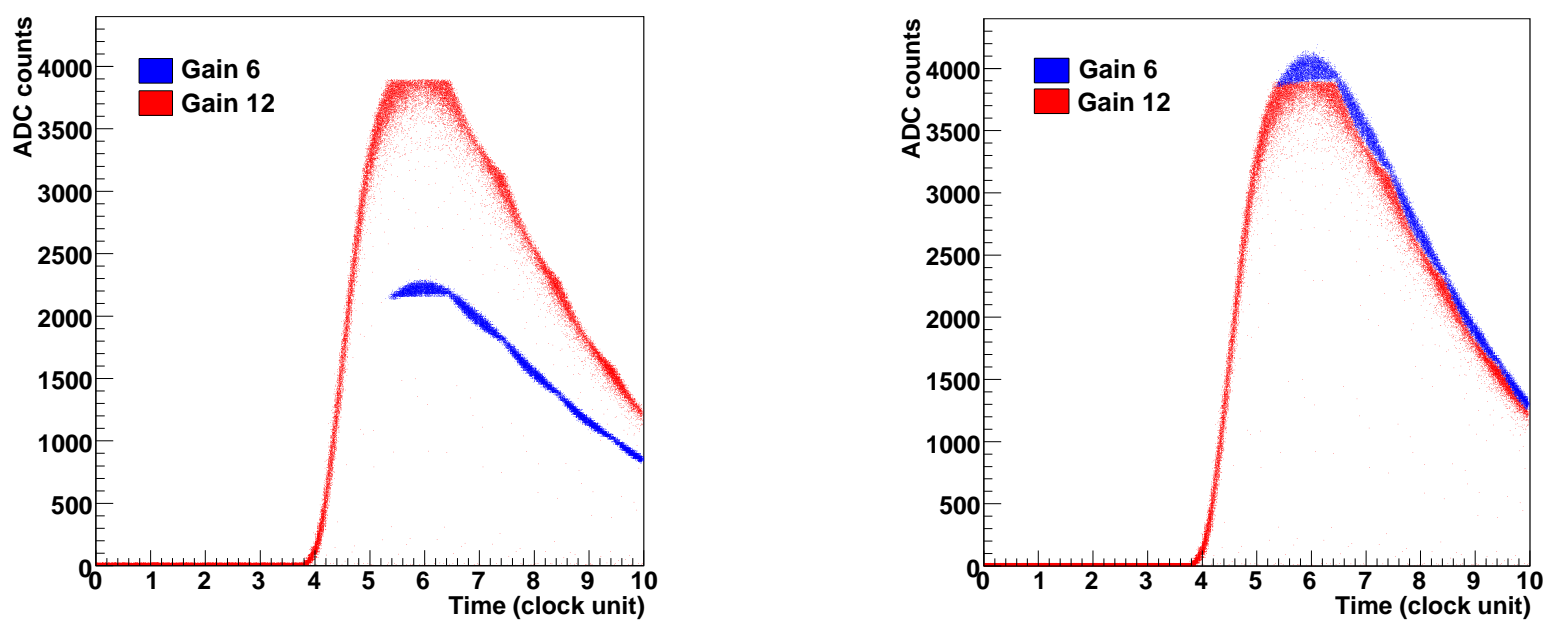

Figure 6.19: Profile of the signal pulse for a crystal exposed to an electron beam of $180 \mathrm{GeV}$, before (left) and after (right) applying the gain ration multiplication. Samples which are recorded in highest gain 12 (red) and in middle gain 6 (blue) are shown [13].

\subsubsection{Check on systematic effects}

The gain ratio have to be known at a few permille precision, which requires the measurement of the gain ratio in situ for each single channel. Therefore, a study on the systematic effects has been performed.

\section{Free and fixed gain mode}

It is obvious that contrary to the normal VFE electronic operation mode where the gain path is chosen automatically for each sample by the selective logic internal to the ADC, the gain ratio measurement requires processing a signal in fixed gain mode, i.e. for a particularly selected gain stage. In principle, there is no way to force the gain of the MGPA directly, but it is possible to stop powering one or two ADC channels of the three that are used and thereby allows the electronics to behave as if the gain was fixed. In this case the data can be recorded only for this particularly chosen gain path. This procedure requires the signal amplitude to be low enough to avoid saturation.

Since the gain ratios are measured from fixed gains and their values will be applied for the reconstruction of the 'true' amplitude, which is recorded with a free gain, it is highly advisable to verify if the use of the fixed gain does not change the output of a VFE channel and thus evaluate any systematic bias which could come from this effect. The verification was performed for one VFE card of the calibration facility at IPNL where $4.1 \mathrm{pC}$ and $11 \mathrm{pC}$ were measured in free and fixed modes for low gain 1 and middle gain 6 , respectively. The results for gain 1 and gain 6 for all five channels of the tested VFE card show that a systematic bias is less than $\sim 0.2 \%$. as is depicted in Fig. 6.20. 


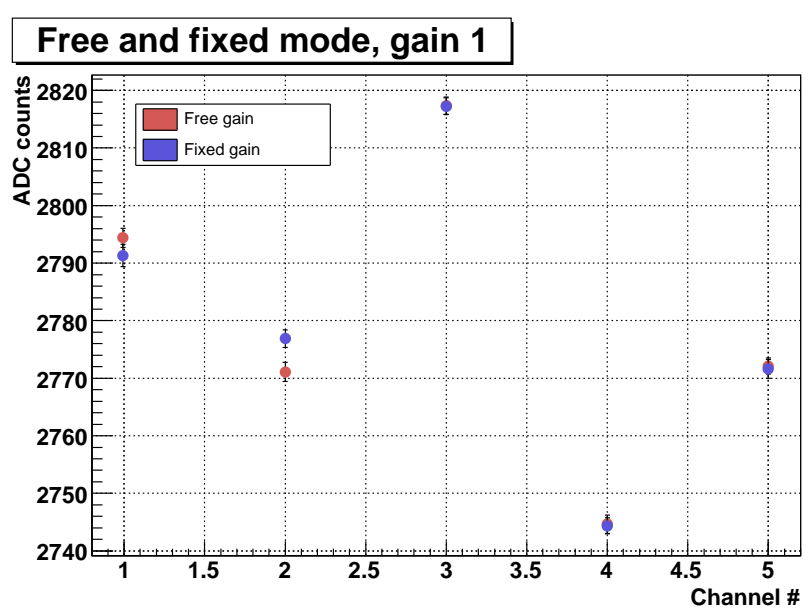

\section{Free and fixed mode, gain 6}

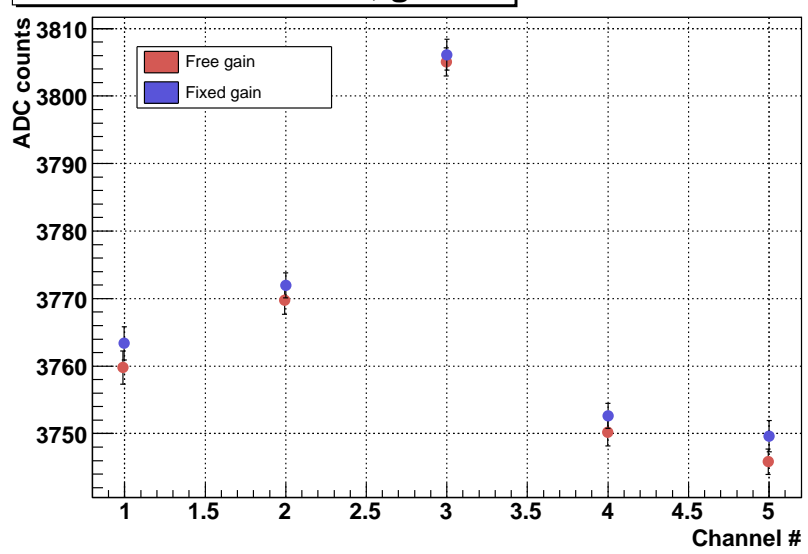

Figure 6.20: Mean value of the the reconstructed amplitude for low gain 1 (top) and middle gain 6 (bottom) measured for one VFE card (5 channels) in free and fixed gain modes. Errors bars indicate the RMS value of the computed amplitudes. Measurements were performed on the VFE calibration bench at IPN Lyon. 


\section{Amplitude reconstruction}

Another contribution to the inaccuracy of the gain ratio determination comes from the systematic errors of the amplitude reconstruction itself. The signal amplitude is generally reconstructed using the weights method which minimizes the contribution of the electronics noise or with an analytic function method. Both techniques were applied on the same data set and it was observed that the difference in computed amplitudes using the weights method and the amplitude reconstruction using the analytic fit method is around $1 \%$ for all the gains. This difference in the ECAL is corrected by a different set of calibration constants. Since the gain ratio is computed as the ratio of the reconstructed amplitudes, the final difference in the gain ratio computation is negligible $(<\sim 0.03 \%)$ as is shown in Fig. 6.21. Therefore, only the weights method was chosen as the main amplitude reconstruction method for all measurements performed in this study.

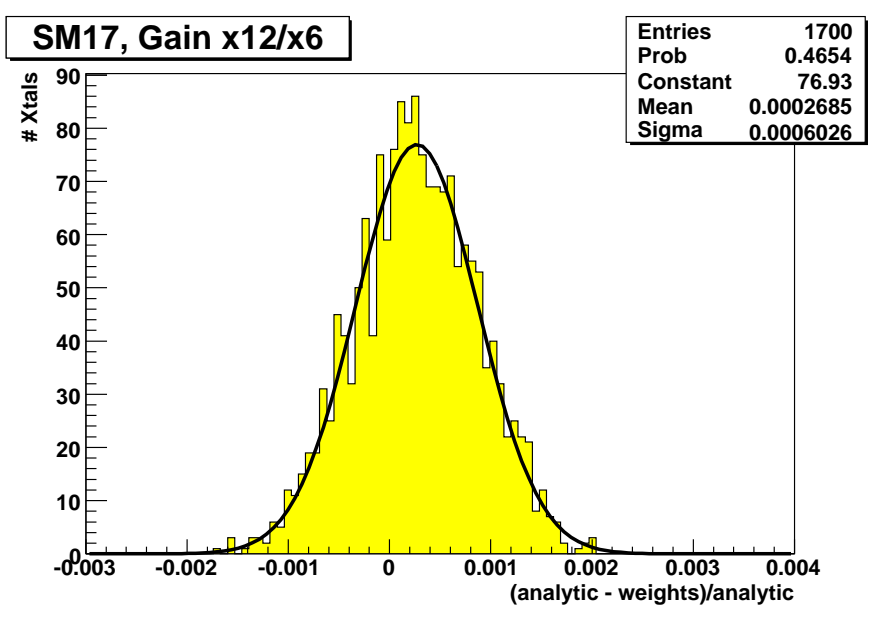

Figure 6.21: Distribution of the relative difference between the gain ratio $12 / 6$ computed using the weights method and analytic fit method. The gain ratios were measured by the test pulse (4.1 pC) on the supermodule SM17 at CERN.

The weights method is used to reconstruct the amplitude which is implemented on 10 digitized samples produced on the output of the ADC. A set of weights, which was used throughout this study consists of $3+7$ weights for gain 12, where the first 3 weights measure the pedestal and another 7 weights are used for samples around the maximum to determine the signal amplitude. The weights were obtained from profile amplitudes for the highest gain 12 and were also used for amplitude reconstruction in middle gain 6 or low gain 1, where only 7 weights around the maximum were used. The pedestal for gains 6 and 1 were determined from dedicated pedestal runs. This supposes that the pulse shape is identical for all three gains. In fact, the pulse shape very slightly differs between the gains which is caused by construction differences among gain stage circuits (a separate amplifier is used for each gain channel). For this reason a cross check study, where the set of weights was determined in gain 6 and then applied on gain 12, was performed. A systematic difference of $1.2 \%$ between the pulse shape profile determined in gain 12 and gain 6 is shown in Fig. 6.22. The bias in the pulse profile results in a $0.1 \%$ shift in the gain ratio measurement as is shown in Fig. 6.23.

Beside the set of weights mentioned above, several other configurations of the weights, such as standard or optimized weights were tested during the test beam campaign. These sets used 


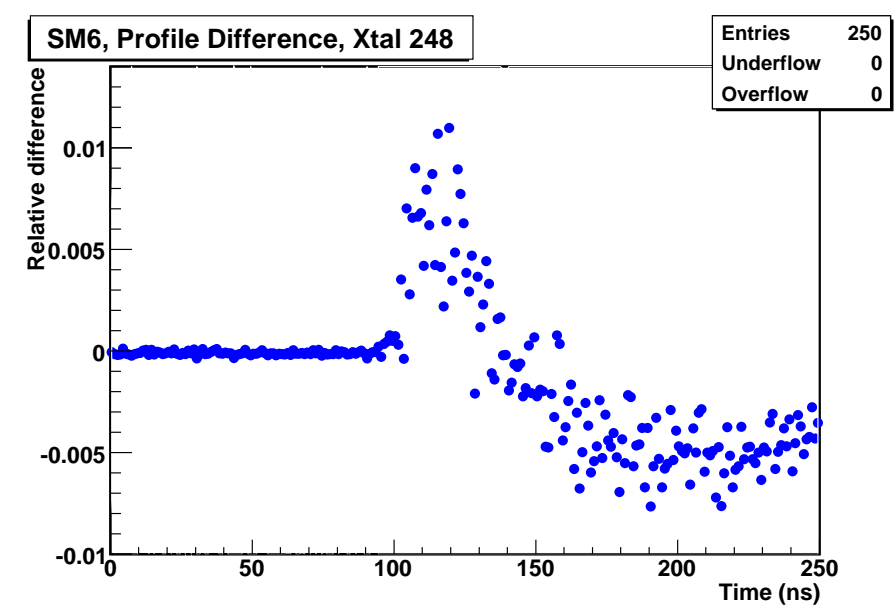

Figure 6.22: Relative difference of the signal pulse profiles determined in gain 12 and gain 6 . The profiles were built for the crystal 248 (supermodule SM6) using electron data.

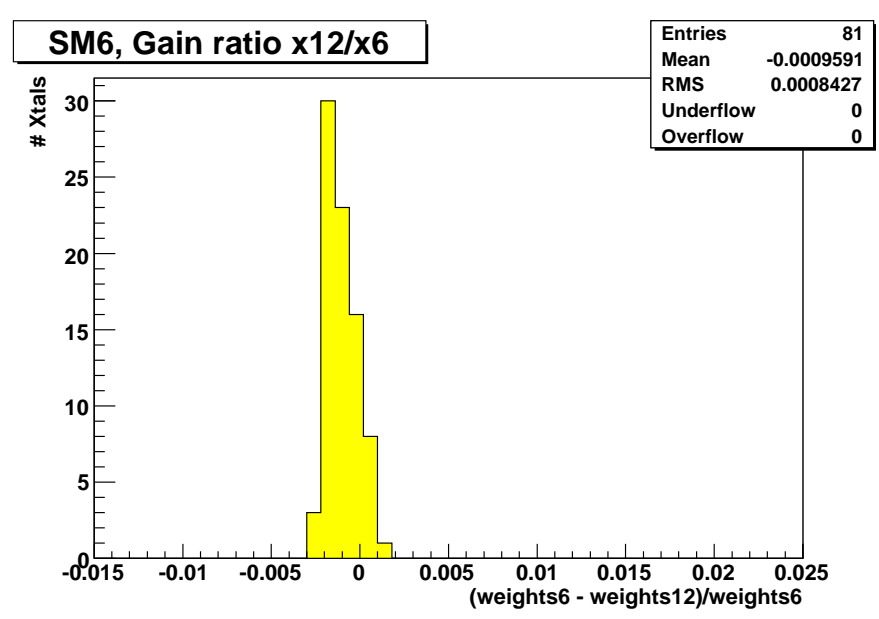

Figure 6.23: Distribution of the relative difference between the gain ratio $12 / 6$ computed using weights determined in gain 12 (weights12) and in gain 6 (weights6). The gain ratios were measured using the electron beam for $9 \times 9$ crystal array on the supermodule SM6 at CERN. 
$3+5$ samples in highest gain 12 and 5 or 4 samples in low gain 6 . To extract the pedestal in gain 6 , the information from presamples in gain 12 or the dedicated pedestal run ahead to the beam run were used. The difference in reconstructed amplitudes using various sets of weights leads to a bias in the gain ratio measurement (see Fig. 6.24) which is, for all the tested cases, smaller than $0.2 \%$.

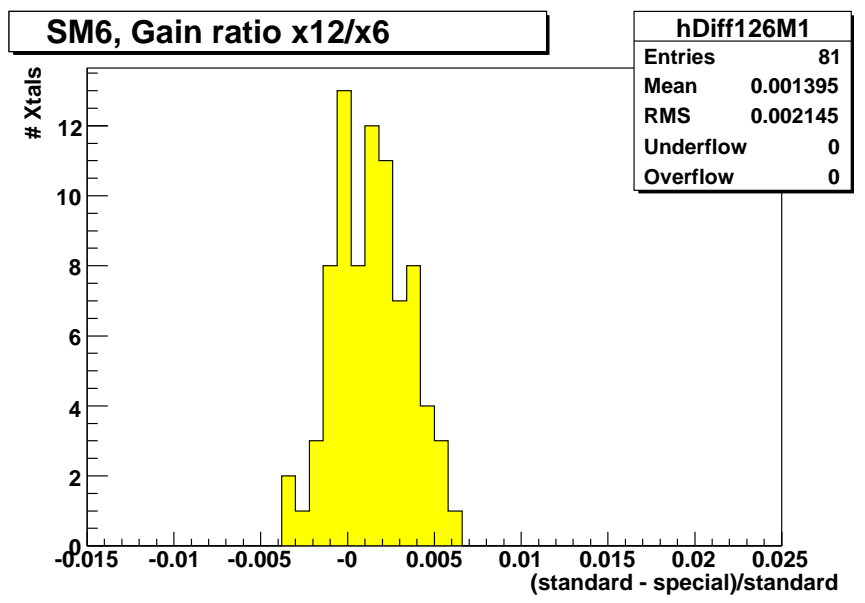

Figure 6.24: Distribution of the relative difference between the gain ratio $12 / 6$ computed using the weights method with $3+7$ weights (special) and with the $3+5$ weights method (standard). The gain ratios were measured using the electron beam for $9 \times 9$ crystal array on the supermodule SM6 at CERN.

Based on the results summarized above, one can conclude that the best way to reduce the bias between the measured and expected gain ratio in the ECAL, is to stay as close as possible to the standard amplitude reconstruction procedure of the ECAL. In the following chapters, different techniques of the gain ratio determination will be presented.

\subsubsection{Gain ratio measured with test pulse}

Two different measurements of the gain ratio have been performed:

- On the fully assembled supermodule SM17 several input charges in a range from 0.6 up to $4.1 \mathrm{pC}$ (corresponding approximately to an energy range from 15 to $105 \mathrm{GeV}$ ) were injected into each channel. Therefore, a test pulse run contains 600 events for each gain. It was found that for all the channels of the supermodule SM17 the mean gain ratio 12/6 is 1.965 with a dispersion within $0.9 \%$.

- On the same channels, but only for one injected charge of $4.1 \mathrm{pC}$, the gain ratio has been determined with a similar level of precision by laboratory test pulse measurements performed on the VFE calibration bench at IPN Lyon. The mean value of the gain ratio $12 / 6$ is 1.938 with a dispersion within $0.9 \%$.

The distribution of the gain ratio for all the 1,700 channels of the supermodule SM17 for an injected charge of $4.1 \mathrm{pC}$ is displayed in Fig. 6.25 for the assembled supermodule and for the test bench results. 


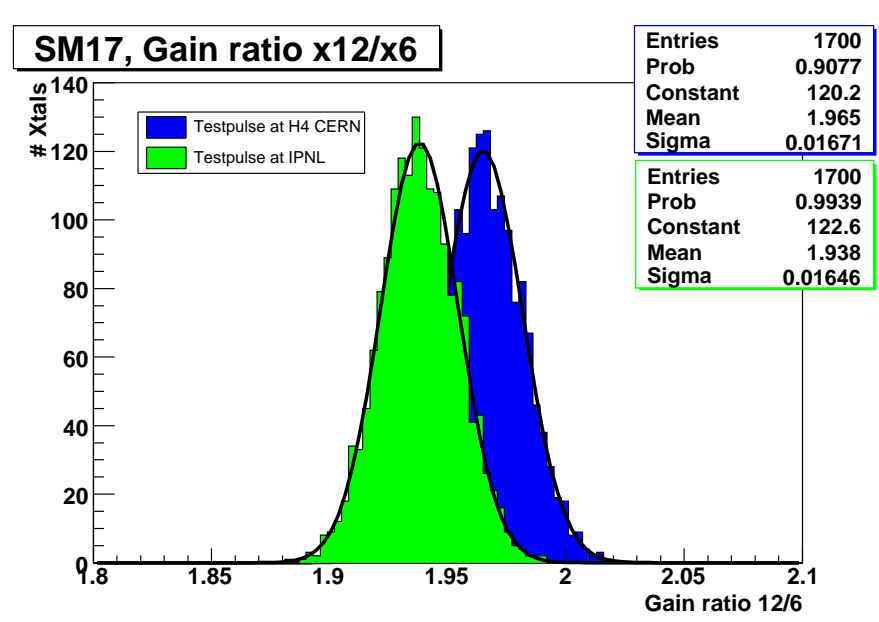

Figure 6.25: Distribution of the gain ratio $12 / 6$ measured using the test pulse on $(4.1 \mathrm{pC})$ the fully equipped supermodule SM17 at CERN (blue) and on the VFE calibration bench at IPN Lyon (green).
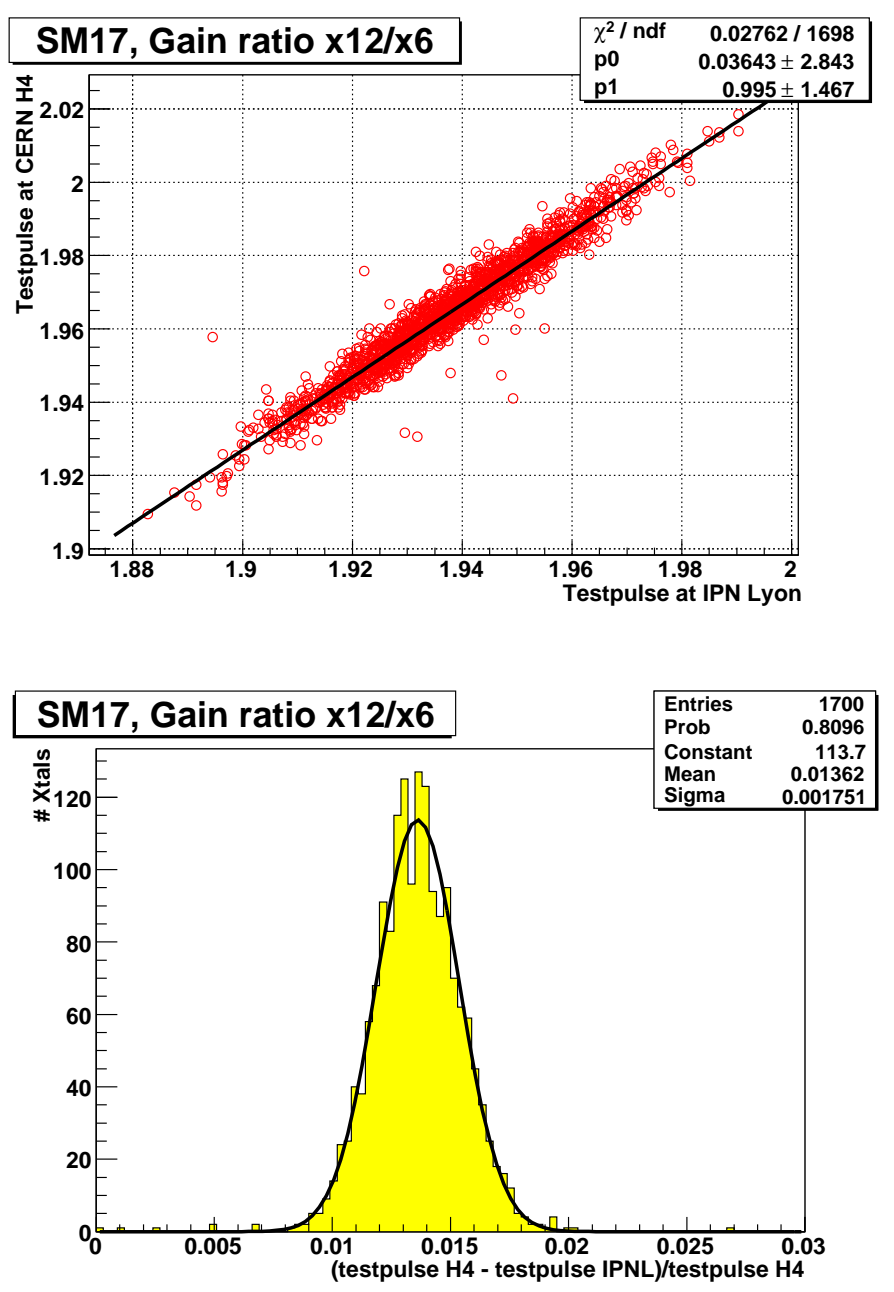

Figure 6.26: Correlation (top) and relative difference (bottom) between gain ratio 12/6 measured by the test pulse on the supermodule SM17 at CERN and on the VFE calibration bench at IPN Lyon. 
Figure 6.26 shows that the gain ratio determined by test pulse on the supermodule and on the VFE calibration bench is well-correlated and consistent in dispersion. On the other hand, a significant systematic shift of $\sim 1.4 \%$ was observed between the means of the two distributions, which could be due to differences in instrumentation which one used. Slight dependence on the injected charge $(\sim-0.05 \% / \mathrm{pC})$ was observed and was confirmed on the supermodule SM5 (see Fig. 6.27) where the test pulse for same injected charges was also taken.

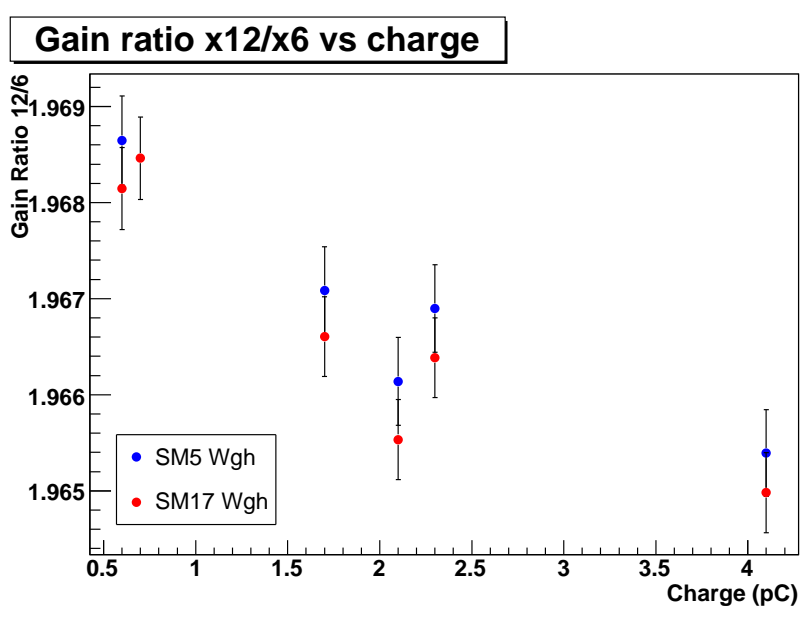

Figure 6.27: Dependence of the mean gain ratio 12/6 on the injected MGPA test pulse charge measured by the test pulse method on the supermodules SM5 and SM17.

However, it should be mentioned that the MGPA test pulse circuit was designed for the testing of the MGPA chip and not for high-precision measurements. The precise charges are not known and are slightly different from channel to channel. Therefore, it is strongly advisable to check the validity of the gain ratio determination with this method. To obtain the value of the gain ratio in data-taking conditions, which will allow the use of the test pulse as a reliable technique of gain ratio measurement for the whole calorimeter, the particle beam must be used in its computation.

\subsubsection{Gain ratio measured with laser}

The gain ratio can also be determined by the monitoring laser system [15], which aims to monitor variations in the light transmission of the crystals due to radiation damage and recovery during the LHC operation. The laser monitoring system was already described in Chap. 2.6.

In order to evaluate the possibility of measuring the gain ratio with a laser signal, several dedicated runs with infrared and blue light pulses have been taken on the supermodule SM6. Laser runs (1,800 events/crystal) were recorded for fixed gain 6 and 12 separately. The amplitudes were reconstructed using the analytical function method and corrected for non-linearity of the PN diodes and APDs and to the laser power fluctuations. Finally, the gain ratios were computed as the ratio of the mean value of the amplitudes over all the events. The intensity of the laser pulse was chosen to be around 3,000 ADC counts for gain 12, which corresponds to an incident electron energy of $\sim 120 \mathrm{GeV}$. 


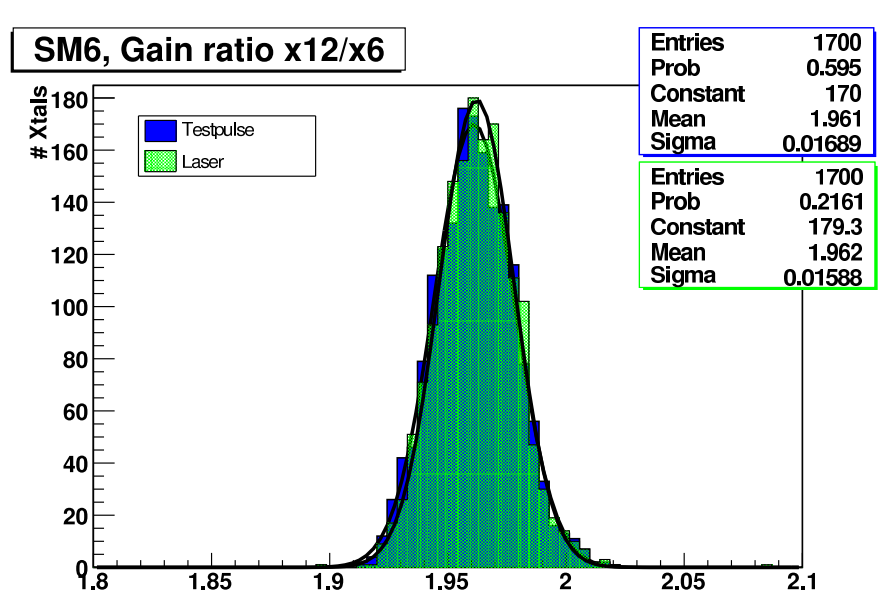

Figure 6.28: Distribution of the gain ratio $12 / 6$ measured using the test pulse $(4.1 \mathrm{pC})$ and laser methods on the fully equipped supermodule SM6 (1,700 channels) at CERN.
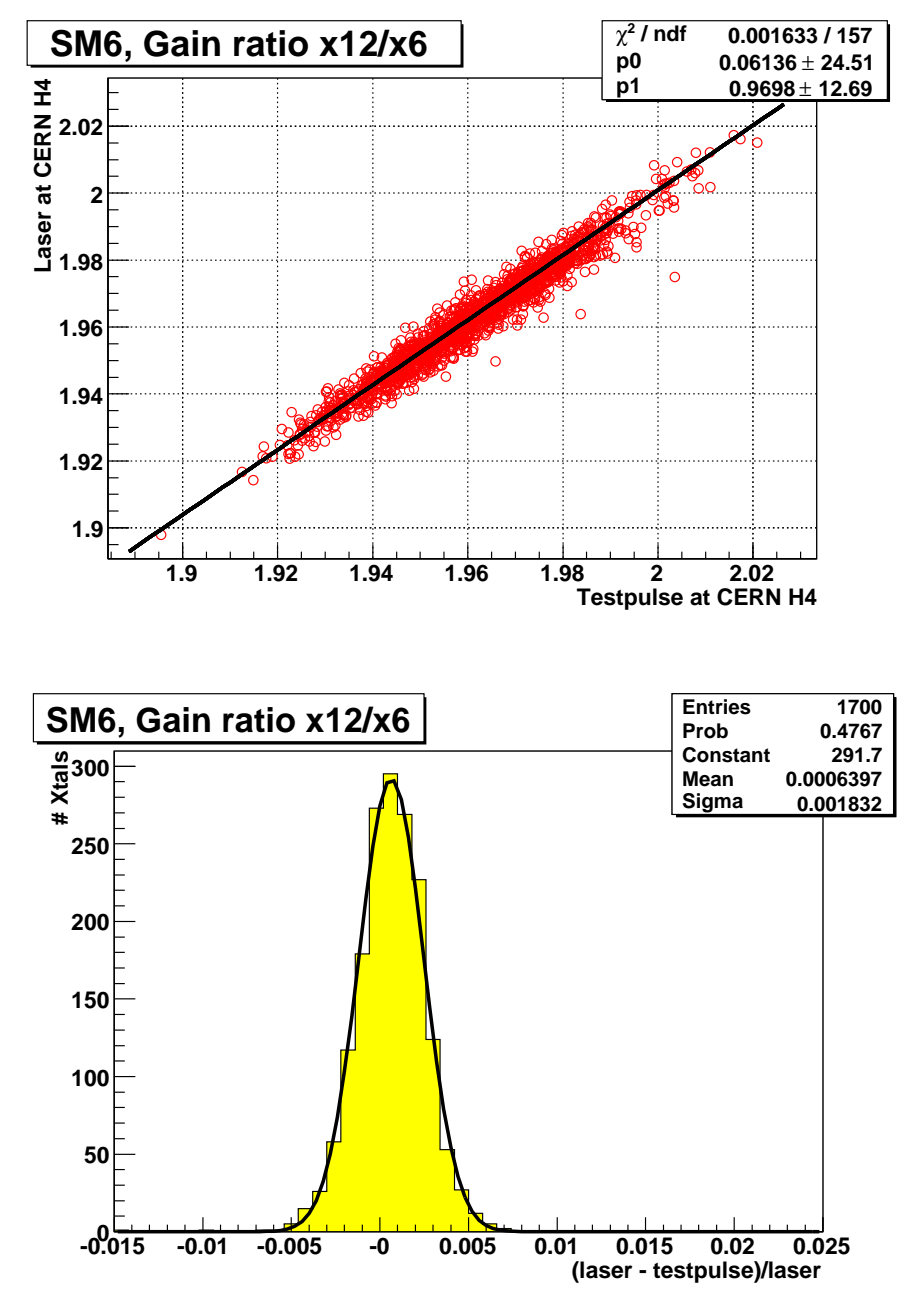

Figure 6.29: Correlation (top) and relative difference (bottom) between gain ratio 12/6 measured by the test pulse and laser methods on the supermodule SM6 (1,700 channels) at CERN. 
Dispersion for the laser as well as for the test pulse is within $0.9 \%$. The mean gain ratio $12 / 6$ measured for all the 1,700 channels of the supermodule SM6 has been measured to 1.962, wellcorrelated and different by only $0.06 \%$ in comparison with the test pulse value of 1.961 measured on the same supermodule with an injected charge of $4.1 \mathrm{pC}$ (see Fig. 6.28 and Fig. 6.29). This comparison study proves that both methods (laser and test pulse) give equivalent results and hence either can be used for gain ratio measurement. The advantage of the test pulse is its simplicity, no additional corrections are needed as in the case of the laser.

\subsubsection{Gain ratio measured with beam}

In order to study gain ratios with beam, high statistics runs ( $>30 \mathrm{k}$ events/crystal) were taken on chosen crystal arrays in different parts of 4 supermodules: SM6, SM13, SM22, and SM25. Each crystal of the arrays, whose sizes were of $3 \times 3,5 \times 5$ or $9 \times 9$ crystals, was exposed to the $90 \mathrm{GeV}$ (SM13 and SM25) or $120 \mathrm{GeV}$ (SM6 and SM22) electron beams in two different modes: a normal mode (using highest gain 12) and then the same crystals were remeasured under identical conditions with fixed middle gain 6 . Moreover, in the case of the supermodule SM22, the data were also taken with fixed gain 1. Since the data from each gain needed to be taken separately, the whole crystal array was measured, for instance in gain 12, and after changing the DAQ system to the gain 6 or 1, the same array was remeasured. This procedure allows to measure the chosen array as quickly as possible during similar beam conditions.

The energy contained in the exposed crystal depends on the impact position of the electron as can be seen from amplitude dependence on the impact position for the $\mathrm{Y}$ hodoscope coordinate displayed in Fig. 6.30 top (blue dots). A similar figure can also be obtained in the $\mathrm{X}$ coordinate. To avoid large energy dispersion, only events in a small region, over which the containment remains almost unchanged, are considered for gain ratio computations. Therefore considered electrons were restricted to a $9 \times 9 \mathrm{~mm}^{2}$ area centered on the point of maximum response in the impacted crystal. Distribution of the reconstructed amplitude as a function of the impact position on the crystal when a hodoscope cut is applied is also displayed in Fig. 6.30 top (red squares). From the figure it is clear that, although a given hodoscope cut reduces the number of events (factor $\sim 5$ ), the number of remaining events is still fully sufficient for proper amplitude calculation. The set of weights needed for a signal amplitude reconstruction is determined from a pulse profile (see Fig. 6.30 bottom) in gain 12 for each crystal separately and then used for amplitude reconstruction of all three gains. An example of distributions of the reconstructed amplitude for one crystal and three gains is shown in Fig. 6.31. Because the amplitude distribution contains a significant left tail due to losses of energy outside the impacted crystal, a fit using the Crystal Ball function, is applied to find a maximum value of the mean amplitude. The ratio of the reconstructed amplitudes gives the gain ratio.

The Crystal Ball function ${ }^{9}$ consists of a Gaussian core portion and a power-law low-end tail and is defined as:

$$
f(x)=N \times \begin{cases}\exp \left(-\frac{(x-\bar{x})^{2}}{2 \sigma^{2}}\right) & \text {-if } \quad \frac{x-\bar{x}}{\sigma}>-\alpha \\ A\left(B-\frac{x-\bar{x}}{\sigma}\right)^{-n} & \text { if } \quad \frac{x-\bar{x}}{\sigma} \leq-\alpha\end{cases}
$$

where

$$
A=\left(\frac{n}{|\alpha|}\right)^{n} \exp -\frac{|\alpha|^{2}}{2} \quad \text { and } \quad B=\frac{n}{|\alpha|}-|\alpha|
$$

$N$ is a normalization factor and $\alpha, n, \bar{x}$ and $\sigma$ are parameters which are fitted with the data.

\footnotetext{
${ }^{9}$ Name given after the Crystal Ball detector, currently located at Mainz Microtron facility [16]
} 

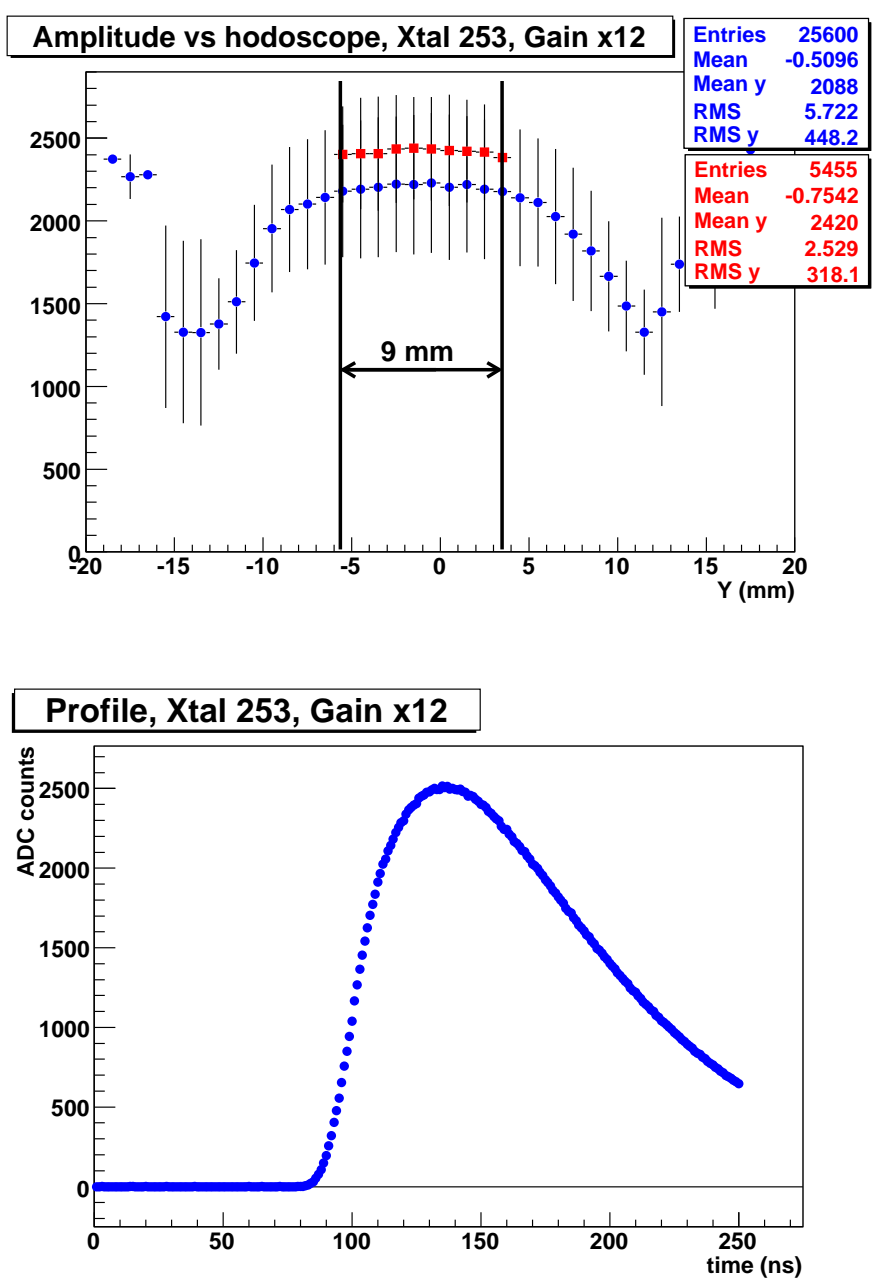

Figure 6.30: Top: Distribution of the reconstructed amplitude in ADC counts as a function of the impact position on the crystal in the Y coordinate for crystal 253, supermodule SM22. The region selected for gain ratio measurement is also indicated. Bottom: Pulse profile in gain 12 (crystal 253, SM22) used for determination of the set of weights for signal amplitude reconstruction. 

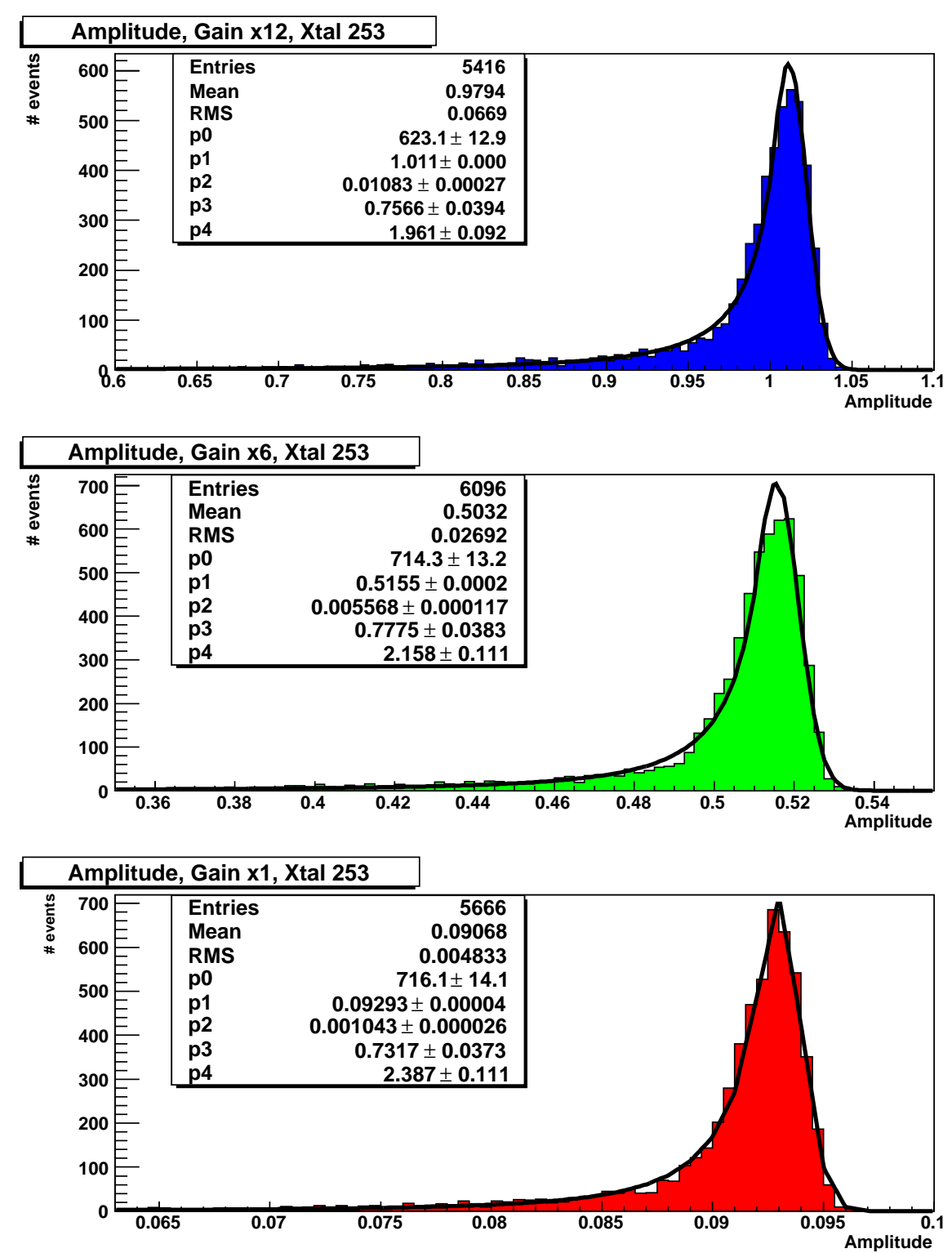

Figure 6.31: Distributions of the reconstructed amplitude over all events for crystal 253, SM22 and three gains. Data were taken in three independent runs and the hodoscope cut of $9 \times 9 \mathrm{~mm}^{2}$ was applied. The figure shows the fit using the Crystal Ball function (see Eqv. 6.10) for finding the maximum amplitude value. Meaning of the fit parameters is: p0 is a normalization factor, p1, p2 are mean and standard deviation of the Gaussian part, and p3, p4 describe the power law tail. 


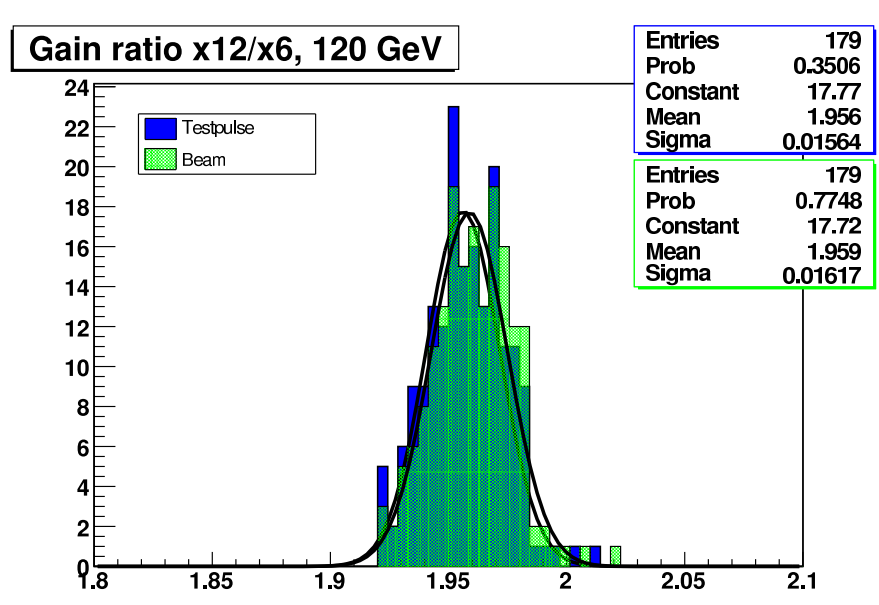

Figure 6.32: Distributions of the gain ratio 12/6 measured using beam (green distribution) and the test pulse methods (blue distribution) for all measured crystals on the supermodules SM6 and SM22 with beam during the test beam campaign 2006 at CERN.
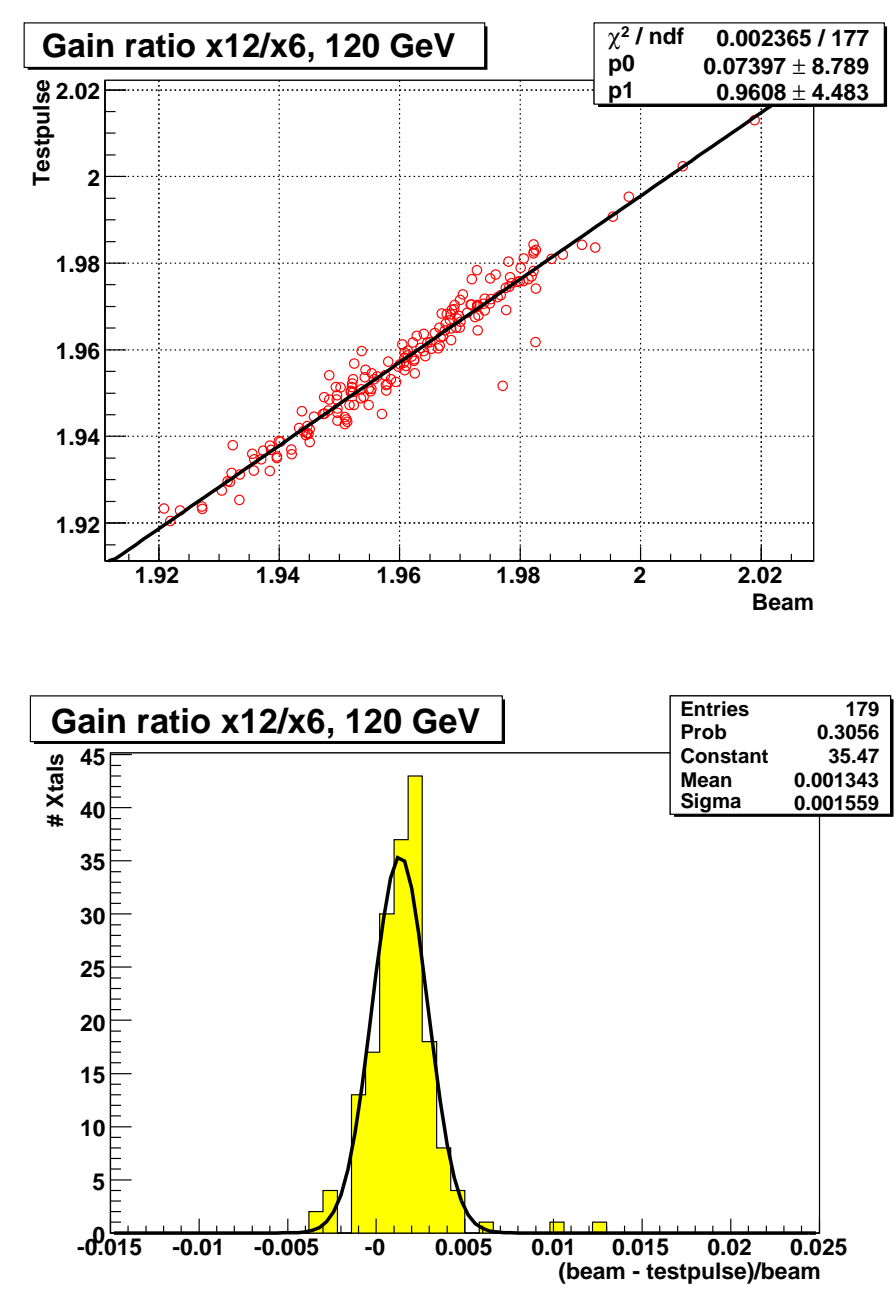

Figure 6.33: Correlation (top) and relative difference (bottom) between the gain ratio 12/6 measured by the test pulse and with an electron beam of $120 \mathrm{GeV}$ at CERN for 179 crystals on the supermodules SM6 and SM22. 


\begin{tabular}{l|l|c|c|c|c|c}
\hline \hline & Method & Module & Array & Gain Ratio & Disp. [\%] & Diff. [\%] \\
\hline \hline SM6 & Beam - 120 GeV & M1 & $9 \times 9$ & 1.963 & 0.91 & 0.19 \\
& Test pulse & M1 & $9 \times 9$ & 1.959 & 0.90 & \\
& Beam - 120 GeV & M4 & $9 \times 9$ & 1.958 & 0.81 & 0.07 \\
& Test pulse & M4 & $9 \times 9$ & 1.957 & 0.81 & \\
\hline SM13 & Beam - 90 GeV & M1 & $3 \times 3$ & 1.969 & 1.45 & 0.38 \\
& Test pulse & M1 & $3 \times 3$ & 1.961 & 1.48 & \\
& Beam - 90 GeV & M2 & $3 \times 3$ & 1.961 & 0.90 & $<0.01$ \\
& Test pulse & M2 & $3 \times 3$ & 1.961 & 0.92 & \\
\hline \multirow{2}{*}{ SM22 } & Beam - 120 GeV & M1 & $5 \times 5$ & 1.960 & 0.71 & 0.24 \\
& Test pulse & M1 & $5 \times 5$ & 1.955 & 0.69 & \\
& Beam - 120 GeV & M1 & $5 \times 5$ & 10.97 & 0.96 & 0.42 \\
& Test pulse & M1 & $5 \times 5$ & 10.92 & 0.98 & \\
\hline SM25 & Beam - 90 GeV & M3 & $3 \times 3$ & 1.977 & 1.00 & 0.75 \\
& Test pulse & M3 & $3 \times 3$ & 1.962 & 0.75 & \\
\hline \hline
\end{tabular}

Table 6.3: Summary results of the gain ratio 12/6 determined with beam and by the test pulse $(4 \mathrm{pC})$ on the four studied supermodules at CERN H4. On SM22 data were also taken in gain 1 , so the results for the gain ratio $12 / 1$ are also included.

The above described procedure was used for all measured data and a mean value of the gain ratio $12 / 6$ measured with the $120 \mathrm{GeV}$ electron beam is 1.959 while the gain ratio determined for the same crystals by use of the test pulse is 1.956, both with a dispersion of approximately $0.8 \%$ (see Fig. 6.32). As can be seen in Fig. 6.33, the gain ratio is well-correlated for all the investigated crystal channels with only a small relative difference of $\sim 0.13 \%$.

Results for each particular crystals array are summarized in Tab. 6.3, which shows the mean values of the gain ratio determined with the beam and its comparison with the test pulse. As clearly seen from the table, the mean value and dispersion over each array have similar values, which demonstrate robustness of technique used. Because the data were taken in different parts of the supermodules, the position dependence of the gain ratio was also considered and was found to be negligible. In addition, on supermodule SM22 data in the lowest gain 1 were measured and results for the gain ratio $12 / 1$ are therefore also included in Tab. 6.3.

Because of the small differences between the gain ratio measured with the test pulse and with the electron beam found in all cases mentioned in Tab. 6.3, the use of the test pulse as a method for reliable gain ratio determination is foreseen for the whole CMS ECAL. Another advantage of the test pulse is that the gain ratio can be measured in situ at any time on all the channels of the calorimeter.

\subsubsection{Energy dependence measurement}

In order to evaluate the influence of beam energy on the gain ratio determination, measurements were performed on the supermodule SM6 with 30,50,90,120, and $150 \mathrm{GeV}$ electron beams in highest gain 12 and middle gain 6 . The crystal set was selected from one row $(\eta=$ const.) 


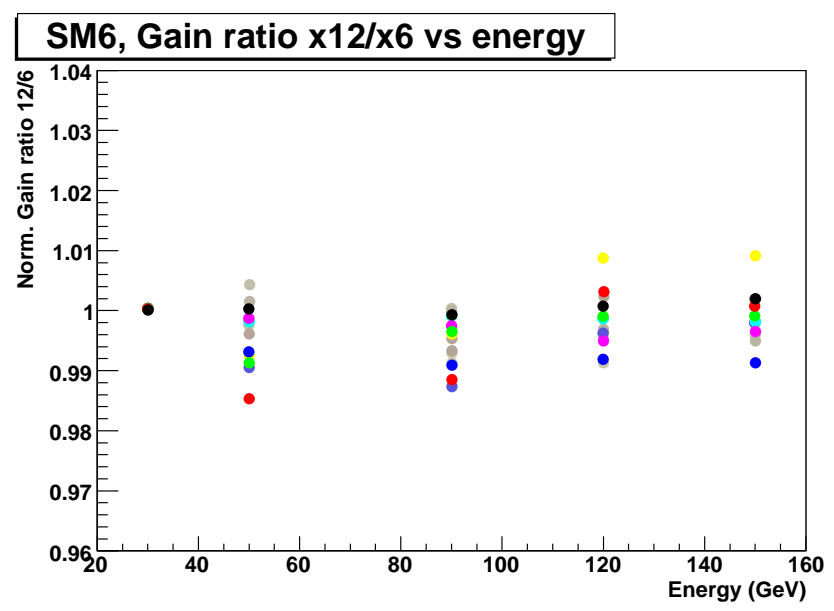

Figure 6.34: The normalized gain ratio $12 / 6$ as a function of the beam energy measured for 15 crystals on the supermodule SM6 et CERN.
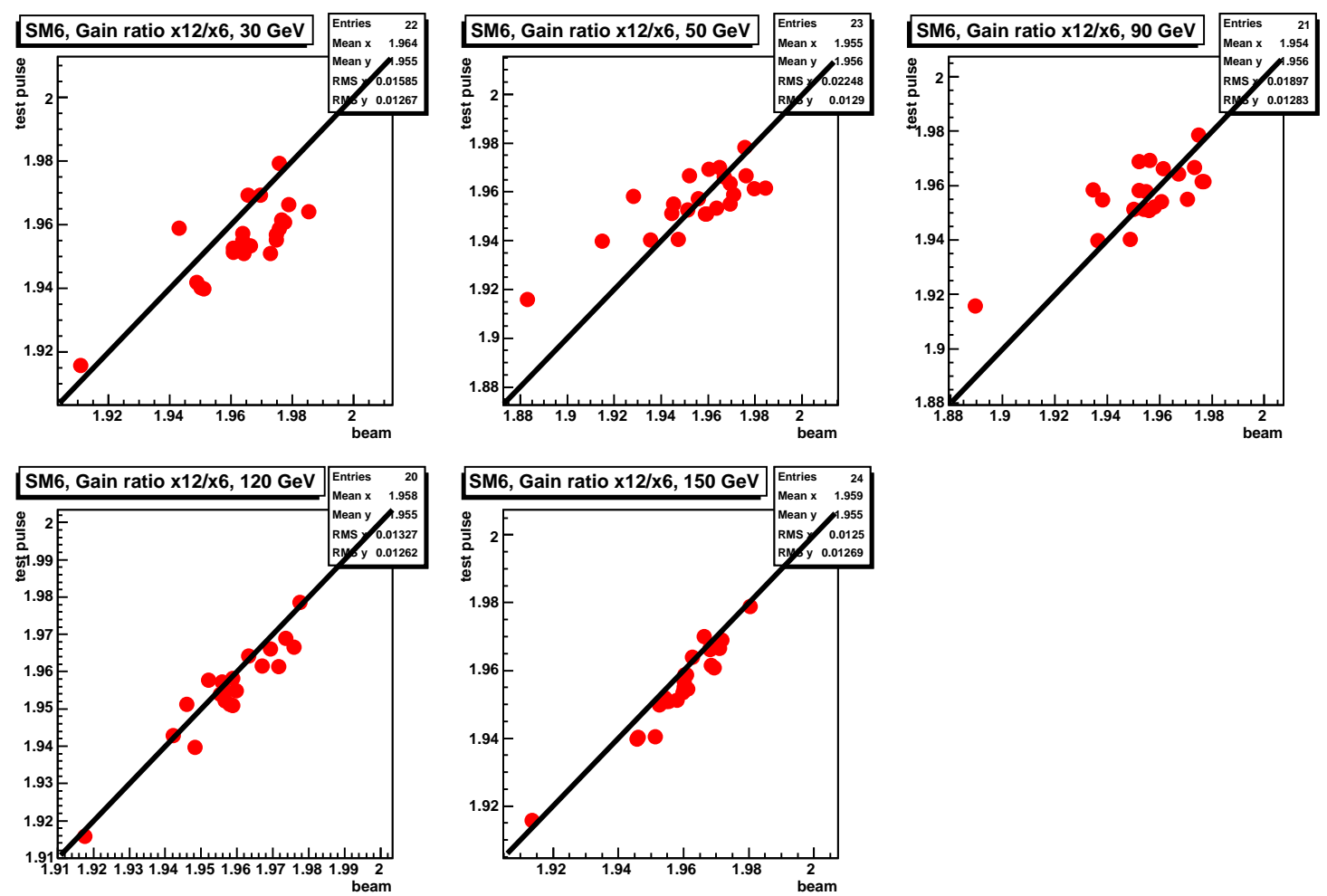

Figure 6.35: Correlation between gain ratio 12/6 measured by the test pulse and with an electron beam for 6 different energies (from 30 to $150 \mathrm{GeV}$ ) on the supermodule SM6 at CERN. The black diagonal lines are only to guide the eye. 
over the all supermodules, so that 5 groups of 4 crystals, with gaps of 7 crystals between each group, were available for the study. The gain ratio was calculated using the same procedure as mentioned earlier. Fig. 6.34 shows that there is no evident trend with increasing energy. The values of the gain ratio seem to be stable for the highest energies $(120$ and $150 \mathrm{GeV})$. A correlation of the gain ratio for all 6 energies obtained with the beam as a function of the test pulse obtained at $4.1 \mathrm{pC}$, corresponding roughly to $105 \mathrm{GeV}$, is displayed in Fig. 6.35. From these comparison plots, the best correlation is for the highest studied energy, $150 \mathrm{GeV}$ (a slope is nearly 1 and offset is almost 0 ). This results is very important, because near this energy the highest gain 12 switches to the middle gain 6 where a precise knowledge of the gain ratio is necessary.

\subsubsection{Stability of the test pulse gain ratio over time}

The stability of the test pulse gain ratio has been studied over the duration of the test beam campaign for the supermodule SM6 ( $\sim 3$ weeks), using a total of 218 runs where the injected charge was 4.1pC. Fig 6.36 top shows the value of the gain ratio $12 / 6$ for the 218 runs representing a time scale, for a typical crystal. The RMS of the distribution of these gain ratio values for each of the 1,700 crystals is shown in Fig 6.36 bottom.

For every testpulse run, 600 events were taken in each gain. Nevertheless, for some runs, due to data acquisition problems or for another reasons, much less events were recorded. Therefore, the data are 'cleaned' by eliminating from consideration, for a given crystal, data from runs with less than 100 events that are needed for a proper amplitude reconstruction. Then, for each gain ratio, a distribution is filled with the RMS of each fitted input gain amplitude divided by the fitted mean, for all the crystals (example for gain 12 shown in Fig 6.37). Data from runs for crystals with a value of the RMS divided by the mean for either input gain lying further than 10 standard deviations from the average value of the global distribution are then eliminated from consideration. This cleaning procedure was found to be sufficient to eliminate the effects of pathological incidents such as tower readout errors, affecting a total of 50 out of the 1,700 crystals. The stability of all gain ratios calculated from their RMS values of the cleaned data-sets is found to be on the order of $0.1 \%$.

\subsubsection{Conclusion on gain ratio studies}

Several methods of gain ratio determination have been investigated on the VFE calibration bench in the laboratory as well as on supermodules. The measurements give comparative results at a precision of $0.2 \%$ for all investigated channels. The test pulse and laser methods of the gain ratio measurement are two alternative techniques giving equivalent results and both can be used as in situ methods. The test pulse gain ratio was also verified for given crystal arrays with gain ratios obtained with the electron beam. These results confirm that the gain ratio measured by the test pulse on the supermodule, due to its simplicity and reliability, is a promising method and could be used for gain ratio determination for the whole CMS electromagnetic calorimeter.

Although the robustness of the gain ratio determination techniques has been shown, the gain ratios measured do not give the optimum energy resolution at high energy. The gain ratios obtained by minimizing the energy resolution at $180 \mathrm{GeV}$ (see Ref. [10] for more details) are systematically shifted by $0.5 \%$. The reason for the shift is probably due to small electronics non-linearity which was observed in the middle gain 6 (see Chap.5.6.6). The linearity problem (determination of the gain ratios at different charge or energy than where they are used) requires to measure non-linearity with an accuracy of few permille. This will be done in situ with the 

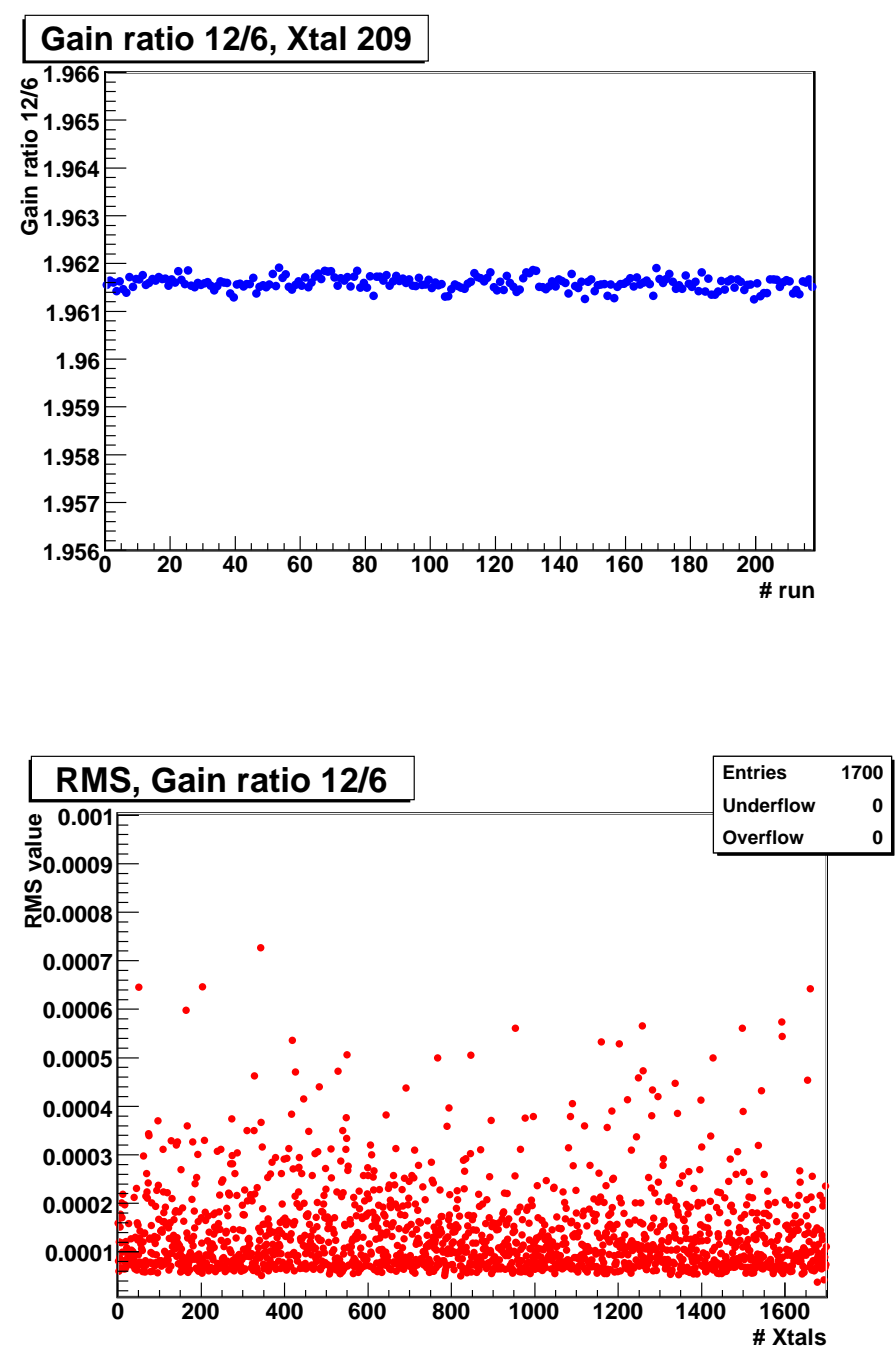

Figure 6.36: Top: The 12/6 gain ratio for a typical crystal as a function of run number measured on the supermodule SM17 over a period of 3 weeks during the test beam campaign. Bottom: the RMS of the distribution of 12/6 gain ratio values for all runs measured on SM17, for each crystal. 


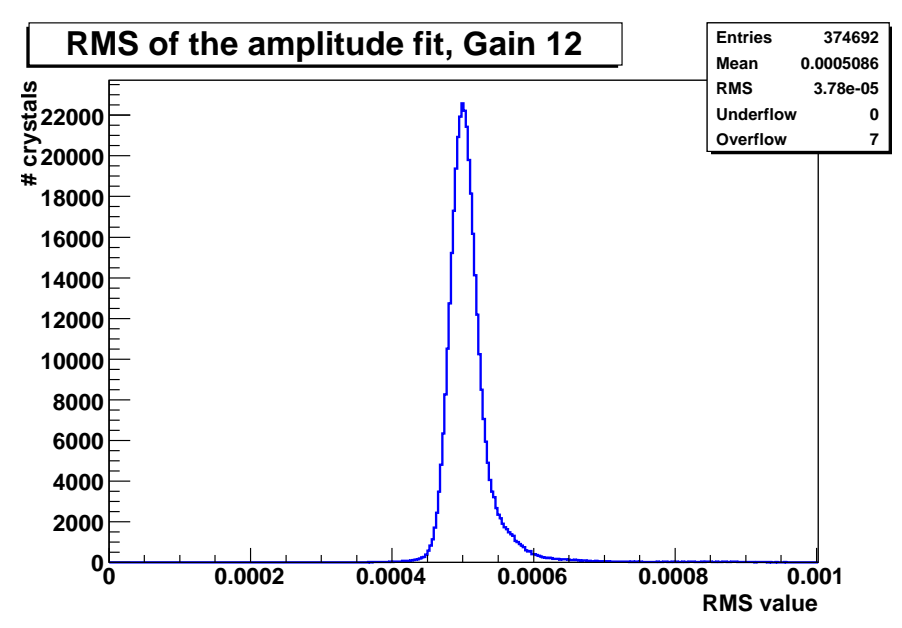

Figure 6.37: Global distribution for all crystals and all runs, of the values of RMS divided by fitted mean, of the gain 12 amplitudes (SM17), used for the data cleaning procedure.

laser system in future.

\subsection{Energy resolution}

The performance of the ECAL measured during the testbeam is well characterised by the energy resolution as a function of the beam energy. Therefore, energy scans, for beam energies from 15 to $250 \mathrm{GeV}$, throughout the different regions on the supermodules were performed. Since the trigger area is only slightly smaller than the front face of a crystal, the incident electrons are spread over this area (so called uniform impact). In this case, the energy resolution can be significantly improved by applying a correction for the varying containment as a function of the incident position. The correction function is determined from test beam data using a $\ln \left(E_{2} / E_{1}\right)$ method $[18,19]$ based on the balance of energy deposited in the surrounding crystals. The method allows to estimate the incident position on the crystals surface using only ECAL information. Fig. 6.38 left shows an example of the energy resolution as a function of energy for the uniform impact measured on the supermodule SM6 using a $3 \times 3$ crystals array centered on crystal 248. The cluster containment correction is applied. For energies higher than $180 \mathrm{GeV}$, the gain ratios determined by the testpulse and corrected on non-linearity effect, are used. The experimental data are fitted by a standard parametrization (see Eqv. 2.1) in order to determine values of the stochastic, constant and noise terms. Then the energy resolution as a function of energy for uniform impact is described by [13]:

$$
\frac{\sigma_{E}}{E}=\frac{3 \%}{\sqrt{E}} \oplus \frac{0.12 G e V}{E} \oplus 0.4 \%,
$$

In Fig. 6.38 right are shown the results for 25 crystals demonstrating consistency in measured results. The parameters of the fit meet the ECAL target specifications (see Tab. 2.1). The resolution is better then $0.5 \%$ for electrons energy greater than $150 \mathrm{GeV}$. These results clearly demonstrate the excellent ECAL performance. 

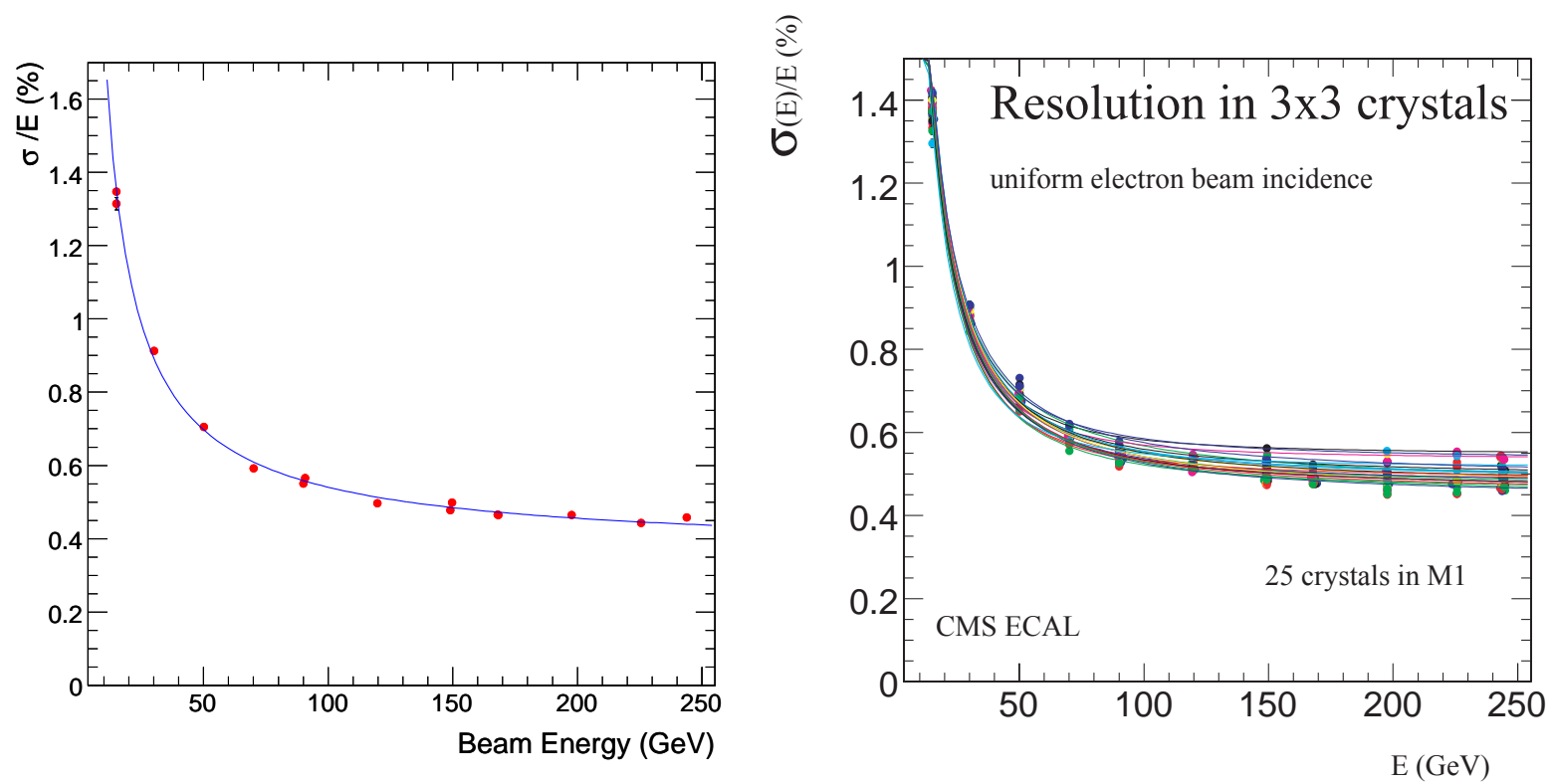

Figure 6.38: Energy resolution as a function of energy for the uniform impact measured on the supermodule SM6 using a $3 \times 3$ crystals array centered on the crystal 248 (left) and for 25 crystals (right). [17].

\subsection{Conclusion}

The tests and intercalibration of nine supermodules during the test beam campaign in 2006 have demonstrated very good performance of the final-state supermodules including their slow control systems that are consistent with the design goals. The front-end electronics have demonstrated very good noise levels and the amplitude reconstruction procedure proved to be effective and robust. The test pulse and the laser methods were found as reliable techniques for in situ precise gain ratio measurement. Generally, the results from tests in high energy electron beams have shown that the expected energy resolution of the ECAL have been achieved. The non-linearity problem has to be addressed in situ. 



\section{Bibliography}

[1] CMS Collaboration, The CMS electromagnetic calorimeter project - Technical Design Report, ser. Technical Design Report CMS. Geneva: CERN, 1997, no. CERN/LHCC 97-33.

[2] P. Adzic et al., "Results of the first performance tests of the CMS electromagnetic calorimeter," CERN, Geneva, Tech. Rep. CMS-NOTE-2005-020, Oct 2005.

[3] "Introduction to the use of the $\mathrm{H} 4$ beam," http://ab-div-atb-ea.web.cern.ch/ab-div-atbea/BeamsAndAreas/h4/H4manual.htm.

[4] G. Daskalakis and I. Van Vulpen, "Position resolution in the CMS ECAL using 2003 testbeam data," CERN, Geneva, Tech. Rep. CMS IN 2004/024, May 2004.

[5] CMS ECAL Group, "Reconstruction of the signal amplitude of the CMS electromagnetic calorimeter," Eur. Phys. J. C, vol. 46 S1, pp. 23-35, 2006.

[6] C. Seez, D. Wardrope, and A. Zabi, "CMS electromagnetic calorimeter: amplitude reconstruction using optimised weights," CERN, Geneva, Tech. Rep. CMS DN-2007/020, Dec 2007.

[7] J. Blaha et al., "Amplitude reconstruction and basic performance of the CMS electromagnetic calorimeter," CERN, Geneva, Tech. Rep. CMS DN-2007/008, Dec 2007.

[8] R. Brunelière et al., "Electronic noise and noise treatment (SM0/FPPA, SM1/MGPA)," in WACH4 Workshop Milano - Ecal Test Beam and Precalibration, Milano, Italy, March 2004.

[9] M. Dejardin, "Analysis of MGPA data (noise, linearity, resolution)," in Ecal Test Beam and Precalibration Meetin, CERN, Dec 2003.

[10] CMS ECAL Group, "Energy resolution of the barrel of the CMS electromagnetic calorimeter," J. Instrum., vol. 2 P04004, pp. 1-18, 2007.

[11] R. Brunelière and A. Zabi, "Amplitude reconstruction in the CMS electromagnetic calorimeter with the weights method," CERN, Geneva, Tech. Rep. CMS AN-2005/050, Nov 2005.

[12] J. Descamps, "Étude et optimisation des performances du calorimètre électromagnétique de l'expérience CMS pour la physique au LHC," Ph.D. dissertation, Université Pierre et Marie Curie, 2007.

[13] Private comunication with P. Jarry, Saclay, DAPNIA.

[14] J. Blaha et al., "Calibration and performance test of the very-front-end electronics for the CMS electromagnetic calorimeter," Nuclear Physics B - Proceedings Supplements, vol. 172, pp. 168-170, October 2007. 
[15] M. Anfrevilleb et al., "Laser monitoring system for the CMS lead tungstate crystal calorimeter," CERN, Geneva, Tech. Rep. CMS-NOTE-2007-028. CERN-CMS-NOTE-2007-028, Nov 2007.

[16] "Crystal ball detector," http://wwwa2.kph.uni-mainz.de/cb/.

[17] P. Jarry, "CMS ECAL resolution measurements on SM06 during TB2006 in H4," CERN, Geneva, Tech. Rep. DN-2008, Note in preparation, 2008.

[18] J. Descamps and P. Jarry, "Periodic position dependence of the energy measured in the CMS electromagnetic calorimeter," CERN, Geneva, Tech. Rep. CMS-NOTE-2006-045. CERNCMS-NOTE-2006-045, Feb 2006.

[19] A. Kyriakis, A. Markou, and E. Petrakou, "Position dependence of energy containment in ECAL barrel crystals from 2006 TestBeam," CERN, Geneva, Tech. Rep. CMS-DN2007/017, May 2008. 


\section{Conclusions}

The work presented in this document is focused on the properties and performance optimization of the very-front-end (VFE) electronics for the electromagnetic calorimeter (ECAL) of the CMS experiment operating on the Large Hadron Collider (LHC) at CERN. The content of the work covers two main complementary parts: the laboratory measurement of all the VFE cards performed at IPN Lyon and the study of the electronics behavior during the test beam experiments with high energy particle beams at CERN.

The laboratory measurement was included in the final stage of the ECAL read-out electronics QA program consisting of the burn-in test and the precise calibration and complete characterization of all the VFE boards intended for the barrel and endcaps, and counting almost 16,000 cards. The main purpose of these tests was to screen-out the problematic pieces from those which fully satisfy the strict collaboration specifications.

Therefore, the systems for burn-in and calibration of the VFE electronics were successfully developed and used at IPN Lyon. The systems are stable and all their performances are well understood. The test program for all the $16.000 \mathrm{VFE}$ boards has been completed. The dispersion in the gains has been found to be small $(\sim 1.2 \%$ for barrel and $\sim 1.4 \%)$ over all measured channels and has shown excellent homogeneity in the electronics production. Noise, linearity and relative gains are complying well with the collaboration specifications. Only around $2 \%$ of boards failed the test criteria and have been consequently rejected. The rest have been assembled into barrel supermodules and endcap supercrystals. The results are registered in a database and can be used for an initial calibration of the ECAL.

The experiments using particle beams have been an unique opportunity to test and evaluate the VFE electronics performance as a part of the fully equipped and functional large-scale system in its final state. The special stress has been put on the evaluation of the electronic noise and its reduction using a suitable amplitude reconstruction method, and finding the most optimal technique to determine the gain ratio which needs to be known precisely in order to obtain a good energy resolution at high energies. This work has shown that complementary linearity studies are needed during the commissioning of the ECAL to reach this goal.

The results obtained during the test beams have been found compatible with results from the laboratory measurement. The front-end electronics have demonstrated very low noise levels and the amplitude reconstruction procedure proved to be effective and robust. The results from tests in high energy electron beams have shown that the expected energy resolution of the ECAL has been achieved.

Finally, it can be concluded that the results obtained in the laboratory at IPN Lyon as well as those at CERN confirm the high quality of the boards production and have shown that the CMS detector specifications have been reached. 
NOM : BLAHA

(avec précision du nom de jeune fille, le cas échéant)

Prénoms : Jan

20 mai 2008

TITRE : Calibration et études des performances de l'électronique frontale du calorimètre électromagnétique de l'expérience CMS auprès du collisionneur LHC

Numéro d'ordre : 63-2008

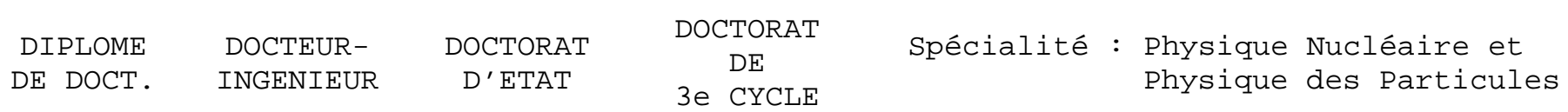

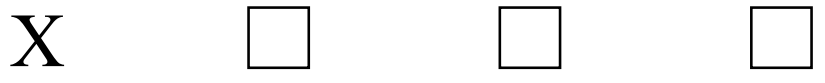

Cote B.I.U. - Lyon : T 50/210/19 / et bis

RESUME :

L'électronique frontale (VFE) traitant les signaux des photodétecteurs du calorimètre électromagnétique de l'expérience CMS a été soumise à un programme étendu de tests pour garantir leur fonctionnalité et fiabilité. Le système de calibration et de caractérisation précise des $\sim 16000$ VFE a été développé et utilisé à IPN Lyon pour contrôler leur qualité et mesurer les constantes de calibration qui serviront au démarrage du calorimètre complet.

En outre, l'évaluation et l'optimisation précises des performances de l'électronique de lecture ont été obtenues avec des données réelles au cours de tests sur des faisceaux de particules au CERN.

Les résultats obtenus sur bancs de tests en laboratoire ou en faisceau confirment l'excellente qualité de la production des cartes électroniques et prouvent que les spécifications du calorimètre ont été atteintes.

MOTS-CLES : LHC - CMS - ECAL - VFE - calibration - Higgs boson - calorimeter

Laboratoire de recherche : Institut de Physique Nucléaire de Lyon

Directeurs de recherches : Houmani El Mamouni -

Président du jury : J. Fay

Composition du jury : M. H.El Mamouni Directeur de Thèse

M. M. Finger Directeur de Thèse

187 pages

M. J. Fay Président du Jury

M P. Jarry Rapporteur

M. V. Vrba Rapporteur

M. J.-P. Ernenwein

M. V. Petråček 